\title{
UICN
}

\section{Conservation pour une ère nouvelle}

Jeffrey A. McNeely et Susan A. Mainka

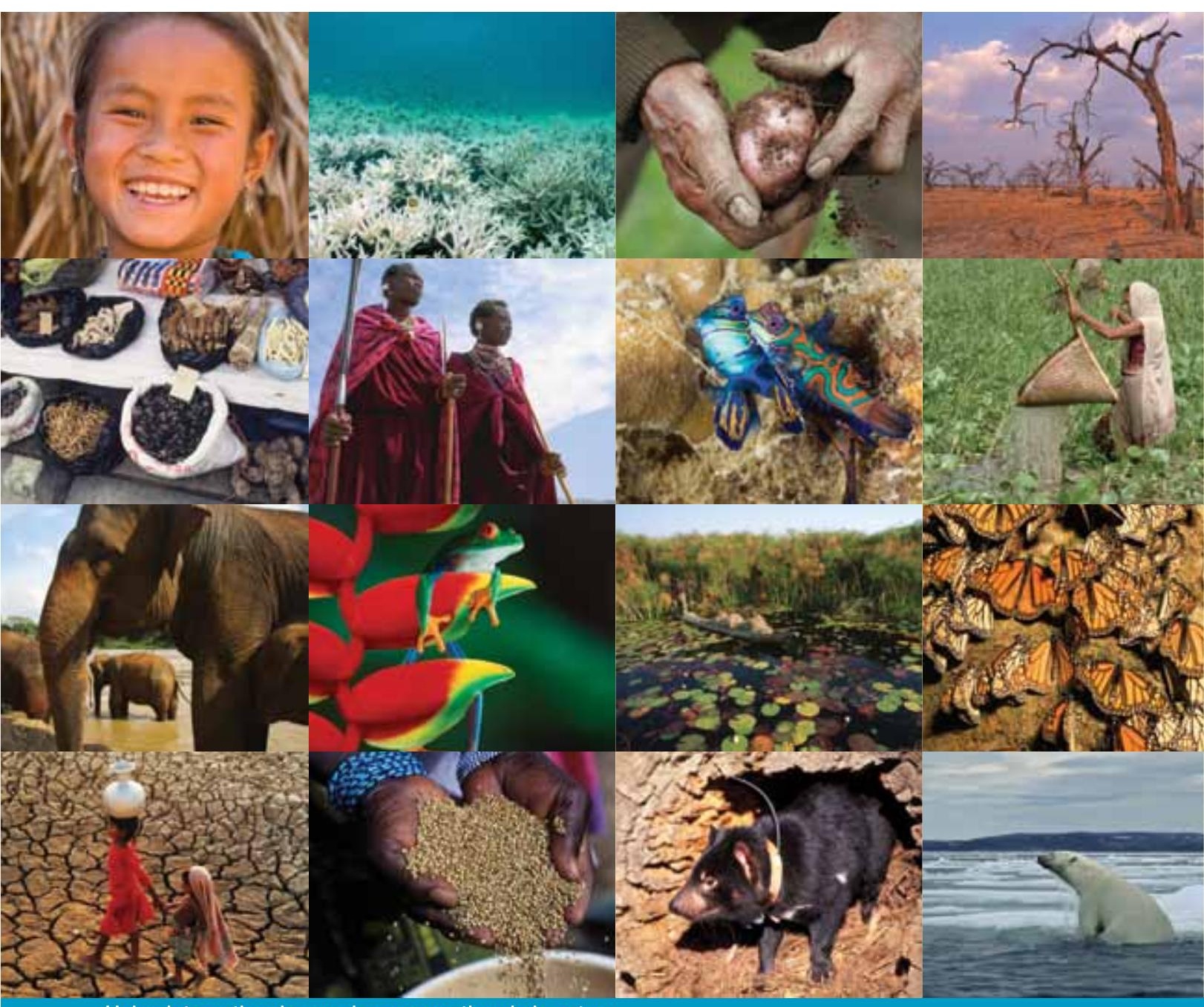

Union internationale pour la conservation de la nature

\begin{tabular}{l|l} 
Ajisty \\
Medi Ambient \\
Ajuntament de Barcelona
\end{tabular} 


\title{
Conservation pour une ère nouvelle
}

\author{
Jeffrey A. McNeely et Susan A. Mainka
}


Traduction : Jacqueline et Jean-Pierre d'Huart

Mise en page et couverture : Charlescannon

Imprimé par : abp project, Suisse

Disponible auprès de :

UICN (Union internationale pour la conservation de la nature) Service des publications

Rue Mauverney 28

1196 Gland, Suisse

Tél +41229990000

Fax +41229990020

books@iucn.org

www.iucn.org/publications

Il existe aussi un catalogue des publications de l'UICN.

Cet ouvrage est imprimé sur papier FSC.
La terminologie géographique employée dans cet ouvrage, de même que sa présentation, ne sont en aucune manière l'expression d'une opinion quelconque de la part de l'UICN sur le statut juridique ou l'autorité de quelque pays, territoire ou région que ce soit, ou sur la délimitation de ses frontières. Les opinions exprimées dans cette publication ne reflètent pas nécessairement celles de l'UICN.

L'UICN rejette toute responsabilité en cas d'erreurs ou d'omissions intervenues lors de la traduction en français de ce document dont la version originale est en anglais.

Publié par : UICN, Gland, Suisse

Droits d'auteur : (C2009 Union internationale pour la conservation de la nature et de ses ressources

La reproduction de cette publication à des fins non commerciales, notamment éducatives, est permise sans autorisation écrite préalable du détenteur des droits d'auteur à condition que la source soit dûment citée.

La reproduction de cette publication à des fins commerciales, notamment en vue de la vente, est interdite sans autorisation écrite préalable du détenteur des droits d'auteur.

Citation : McNeely, J.A. et S.A. Mainka. 2009. Conservation pour une ère nouvelle. UICN, Gland, Suisse. 238pp. 


\section{Table des matières}

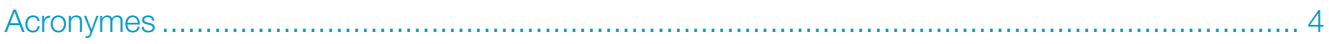

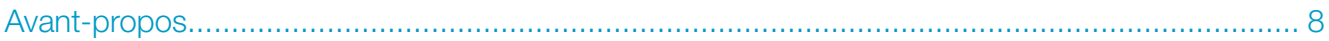

Préface

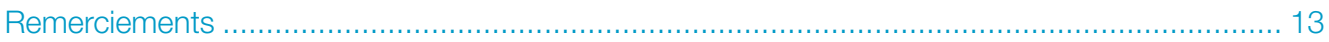

1. Le Forum de Barcelone : Un monde divers et durable ……………………………………............ 14

2. L'éthique de la conservation du $21^{\text {ème }}$ siècle ...................................................................................... 28

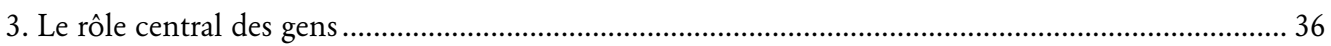

4. Services écosystémiques : les avantages que les hommes reçoivent de la nature................................ 44

5. Changements climatiques et biodiversité .................................................................................... 52

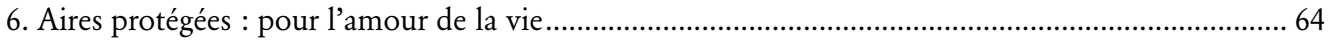

7. Conservation des espèces : Défis d'aujourd'hui.............................................................................72

8. Un avenir après le pétrole : qu'est-ce que cela signifie pour la conservation ? .................................... 82

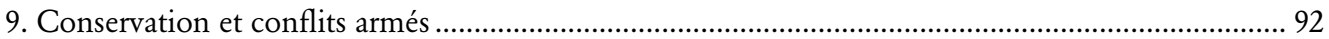

10. Affronter les catastrophes : réflexions sur la restauration post-catastrophe

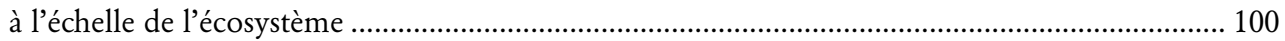

11. Santé humaine et biodiversité : que peut faire la conservation ?................................................... 106

12. Développer une économie « verte " .......................................................................................... 114

13. Technologie et conservation ................................................................................................... 122

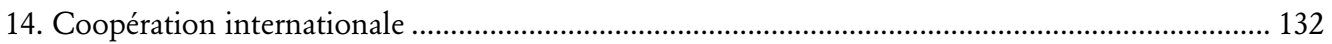

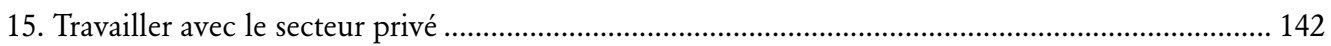

16. Systèmes forestiers : voir la forêt et l'arbre............................................................................. 150

17. Systèmes marins : orienter la conservation vers la mer................................................................ 158

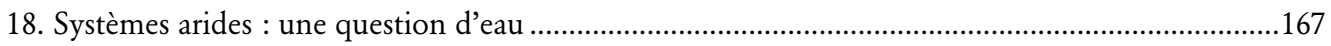

19. Systèmes d'eau douce : gérer les flux pour les hommes et pour la nature ……………………......174

20. Systèmes agricoles : la biodiversité dans des paysages domestiqués.............................................. 182

21. Systèmes urbains : la conservation dans la ville ……………………………………………...... 190

22. Une feuille de route pour Conservation pour une ère nouvelle.................................................... 198

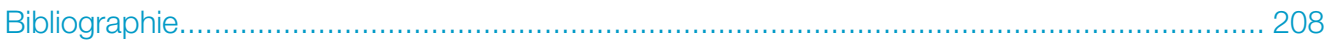




\section{Acronymes}

ACR Accords commerciaux régionaux

AIE Agence internationale de l'énergie

AME Accord multilatéral sur

l'environnement

AMP Aire marine protégée

AO Appel d'offre

AP Aire protégée

APA Accès et partage des avantages

APD Aide publique au développement

APV Accord de partenariat volontaire

BAD Banque africaine de développement

BBOP Business Biodiversity Offset Programme

BDMAP Base de données mondiale sur les aires protégées

BIP Partenariat relatif aux indicateurs de biodiversité

BM Banque mondiale

CCAD Comisión Centroamericana des Ambiente y Desarollo

CCD Convention des Nations Unies sur la lutte contre la désertification dans les pays gravement touchés par la sécheresse et/ou la désertification, en particulier en Afrique

CCNUCC Convention-cadre des Nations Unies sur les changements climatiques

CDB Convention sur la diversité biologique

CDD Commission sur le développement durable

CDDE Commission UICN du droit de l'environnement

CDE Centre du droit de l'environnement
CEC Commission UICN de l'éducation et de la communication

CEFDHAC Conférence sur les Ecosystèmes de Forêts Denses et Humides d'Afrique Centrale

CGE Commission UICN de la gestion des écosystèmes

CICTA Commission internationale pour la conservation des thonidés de l'Atlantique

CIFOR Centre International de Recherche sur les Forêts

CIMM Conseil international des mines et métaux

CITES Convention sur le commerce international d'espèces de faune et de flore menacées

CMAP Commission mondiale des aires protégées

CMED Commission mondiale pour l'environnement et le développement

CMN Congrès mondial de la nature

CMS Convention sur les espèces migratrices

CNUCED Conférence des Nations Unies sur le commerce et le développement

CNUDM Convention des Nations Unies sur le droit de la mer (UNCLOS)

COP Conférence des Parties

CPEES Commission UICN des politiques environnementales, économiques et sociales

CPF Partenariat de collaboration sur les forêts

CSC Capture et stockage du carbone 


\begin{tabular}{|c|c|c|c|}
\hline CSE & Commission de la sauvegarde des espèces & GWP & Partenariat global pour l'eau \\
\hline DAC & $\begin{array}{l}\text { Comité de coopération au } \\
\text { développement (de l’OCDE) }\end{array}$ & ICRAF & $\begin{array}{l}\text { Conseil international de recherches en } \\
\text { agroforesterie }\end{array}$ \\
\hline DALY & Survie ajustée pour l'incapacité & IDE & Investissement direct à l'étranger \\
\hline $\mathrm{DBO}$ & Demande biologique d'oxygène & IDH & Indicateur de développement humain \\
\hline $\mathrm{EbA}$ & Adaptation écosystémique & IIDD & Institut international du \\
\hline EIE & Etude d'impact sur l'environnement & & développement durable \\
\hline EM & $\begin{array}{l}\text { Évaluation des écosystèmes pour le } \\
\text { millénaire }\end{array}$ & IIED & $\begin{array}{l}\text { Institut international pour } \\
\text { l'environnement et le développement }\end{array}$ \\
\hline FAO & $\begin{array}{l}\text { Organisation des Nations Unies pour } \\
\text { l'alimentation et l'agriculture }\end{array}$ & IOSEA & $\begin{array}{l}\text { Mémorandum d'Accord sur la } \\
\text { Conservation et la Gestion des Tortues }\end{array}$ \\
\hline FEM & Fonds pour l'environnement mondial & & $\begin{array}{l}\text { Marines et de leurs habitats dans } \\
\text { l'océan Indien et l'Asie du Sud-est }\end{array}$ \\
\hline FLEG & $\begin{array}{l}\text { Application des réglementations } \\
\text { forestières et gouvernance }\end{array}$ & IRC & $\begin{array}{l}\text { Centre International de l'Eau et } \\
\text { l'Assainissement }\end{array}$ \\
\hline FLEGT & $\begin{array}{l}\text { Application des réglementations } \\
\text { forestières, gouvernance et échanges } \\
\text { commerciaux }\end{array}$ & ISSC-MAP & $\begin{array}{l}\text { Norme internationale relative à la } \\
\text { collecte sauvage soutenable de plantes } \\
\text { médicinales et aromatiques }\end{array}$ \\
\hline FNUF & Forum des Nations Unies sur les forêts & IUFRO & Union internationale des instituts de \\
\hline GBO & Perspectives mondiales en matière de & & recherches forestières \\
\hline & diversité biologique & IUU & Illicite, non déclarée, non réglementée \\
\hline GDF & Gestion durable des forêts & & (pêche) \\
\hline GES & Gaz à effet de serre & $\mathrm{KBA}$ & Zone(s) clé pour la biodiversité \\
\hline GIEC & $\begin{array}{l}\text { Groupe d'experts intergouvernemental } \\
\text { sur l'évolution du climat }\end{array}$ & MDP & $\begin{array}{l}\text { Mécanisme pour le développement } \\
\text { propre }\end{array}$ \\
\hline GIRE & Gestion intégrée des ressources en eau & MNC & Sociétés multinationales \\
\hline GISP & $\begin{array}{l}\text { Programme mondial sur les espèces } \\
\text { envahissantes }\end{array}$ & NBSAP & $\begin{array}{l}\text { Stratégie et plan d'action nationaux } \\
\text { pour la biodiversité }\end{array}$ \\
\hline GSEE & $\begin{array}{l}\text { Groupe CSE/UICN de spécialistes des } \\
\text { espèces envahissantes }\end{array}$ & NEPAD & $\begin{array}{l}\text { Nouveau partenariat pour le } \\
\text { développement de l'Afrique }\end{array}$ \\
\hline GSTC & $\begin{array}{l}\text { Critères Mondiaux de Tourisme } \\
\text { Durable (Le Partenariat pour les) }\end{array}$ & NU-DAES & $\begin{array}{l}\text { Département des affaires économiques } \\
\text { et sociales des Nations Unies }\end{array}$ \\
\hline GTPAN & $\begin{array}{l}\text { Réseau mondial des aires protégées } \\
\text { transfrontalières }\end{array}$ & OCDE & $\begin{array}{l}\text { Organisation de coopération et de } \\
\text { développement économiques }\end{array}$ \\
\hline
\end{tabular}


OCDE-CADComité d'aide au développement de l'OCDE

OGM Organisme(s) génétiquement modifié(s)

OIBT Organisation internationale des bois tropicaux

OMC Organisation mondiale du commerce

OMD Objectif(s) du millénaire pour le développement

OMM Organisation météorologique mondiale

OMS Organisation mondiale de la santé

OMT Organisation mondiale du tourisme

PALNet Réseau d'apprentissage sur les aires protégées

PCR Réaction en chaîne par polymérase

PEBLDS Stratégie paneuropéenne pour la protection de la biodiversité et paysagère

PES Paiement pour les services fournis par les écosystèmes

PFD Partenariats forestiers en développement

PIB Produit intérieur brut

PME Petites et moyennes entreprises

PNUD Programme des Nations Unies pour le développement

PNUE Programme des Nations Unies pour l'environnement

REDD Réduction des émissions issues de la déforestation et de la dégradation des forêts

RSB Table ronde sur les biocarburants durables

RSE Responsabilité sociale des entreprises

SCM Système commercial multilatéral
SE

SEI

SIS

SMDD

TEEB

TCI

TI

UICN Union internationale pour la conservation de la nature

UNCLOS Convention des Nations Unies sur le droit de la mer (CNUDM)

UN-DAES Département des Affaires économiques et sociales des Nations Unies

UNECE Commission économique des Nations Unies pour l'Europe

UNESCO Organisation des Nations Unies pour l'éducation, la science et la culture

WBCSD Conseil mondial des affaires sur le développement durable

WGWAP Comité consultatif sur la baleine grise occidentale

WRI World Resources Institute

WWF Fonds mondial pour la nature

ZLEA Zone de libre-échange des Amériques 


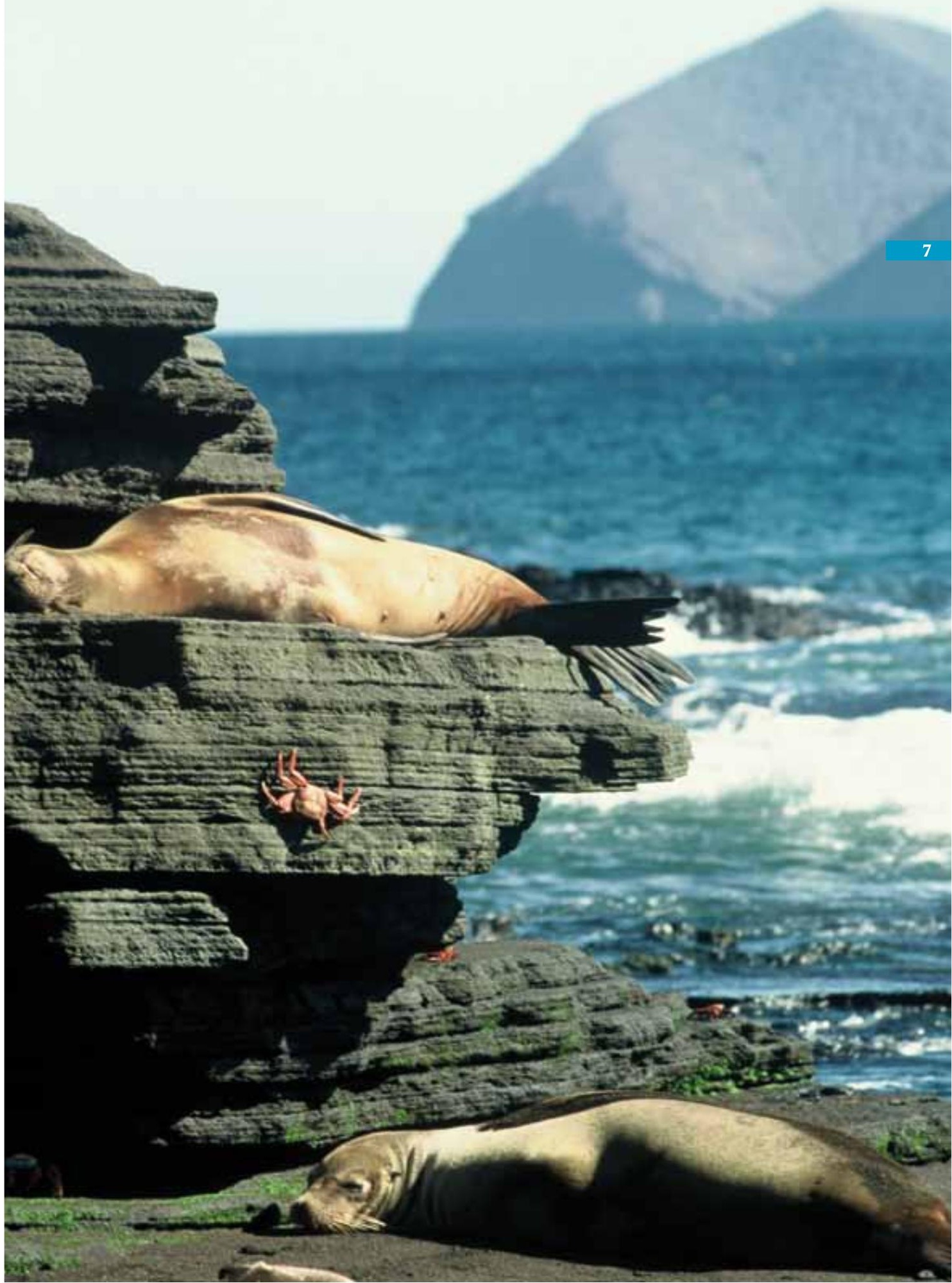




\section{Avant-propos}

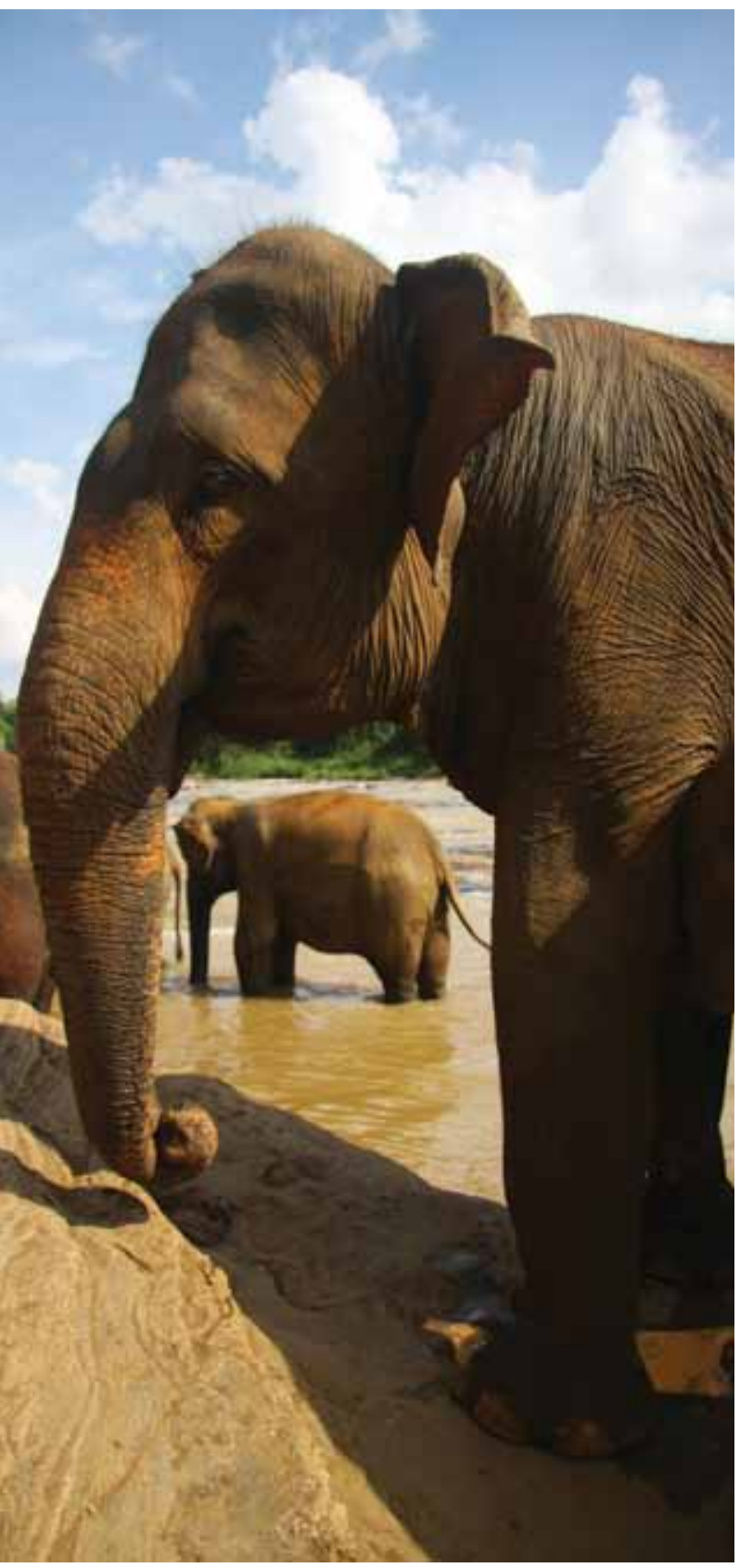

Des conversations ne suffisent

pas pour faire de la bonne

conservation sur le terrain. Pas

même les bonnes conversations.

Pour que la conservation produise de bons résultats, il faut travailler dur, avant tout sur le terrain. La vie d'un conservationniste est faite de longues heures de voyage pour arriver sur place, de plus longues heures d'observations, de plus longues heures encore d'analyse, de réflexion et de synthèse - et puis encore des heures en laboratoire ou à la bibliothèque, pour se documenter et communiquer. Cela ne veut pas dire qu'une vie passée dans la conservation est fastidieuse ; en fait, par nature, elle est plaisante, mais ce qui est d'abord plaisant, c'est la nature.

Néanmoins, pour toutes les professions concernées, les progrès des connaissances en matière de conservation dépendent du partage, de la critique, du questionnement, de l'affinement et du ciselage d'idées générées par la recherche et tournées vers l'action, en interaction permanente avec des collègues et des partenaires de disciplines connexes et d'autres. Les moyens de communication modernes ont révolutionné les possibilités de ces partages mais en fin de compte, 
rien ne peut remplacer les rencontres face à face, quand chercheurs et praticiens peuvent se voir et échanger des informations sur ce qu'ils font.

La rencontre par excellence est, bien entendu, le congrès mondial périodique qui réunit des professionnels en assez grand nombre pour délimiter les frontières actuelles des connaissances, créer des repères collectifs et établir des connections et des réseaux pour de futurs travaux. Et il n'y a rien de tel qu'un congrès comme celui-là pour mobiliser l'esprit d'un professionnel en lui offrant un public, un lieu et un délai limite pour présenter ses derniers progrès et identifier les opportunités pour les prochains pas de géants que vont devoir faire nos connaissances et notre compréhension.

En d'autres termes, si les conversations ne sont pas suffisantes en elles-mêmes, une bonne conservation a quand même besoin, à l'occasion, de bonnes conversations.

C'est pourquoi, en 1996, l'UICN a décidé d'élargir l'Assemblée de ses Membres qu'elle convoquait tous les quatre ans pour en faire le Congrès mondial de la nature $(\mathrm{CMN})$. Celui-ci réunit les conservationnistes les plus éminents de toutes les régions et de toutes les nations afin qu'ils discutent et partagent leur travail et leurs découvertes. Le CMN 2008, qui s'est tenu à Barcelone en octobre et qui a réuni plus de 7000 participants, fut la plus grande conférence civile jamais conviée dans le domaine de l'environnement. Ce fut une occasion inestimable pour les Membres, les Commissions et les partenaires de l'UICN, de même que pour des particuliers, des ONG, des gouvernements et des entreprises de débattre des nombreuses questions qui préoccupent aujourd'hui la communauté de la conservation.

\section{Ce livre, Conservation pour une ère nouvelle,} présente une synthèse de ces discussions tous azimuts. Il examine l'état de nos ressources naturelles aujourd'hui, celui de la conservation, et les tendances actuelles. Il souligne le consensus très net auquel a abouti le Congrès : le constat que le travail sur les espèces, les aires protégées et les écosystèmes qui est au cœur de l'UICN sera un élément toujours plus critique de toute stratégie sociétale pouvant conduire à un avenir durable. Et il décrit comment la communauté de la conservation répond à ce défi - et à cette opportunité.

La richesse du contenu de ce livre et l'accessibilité de son langage, de sa structure et de sa présentation devraient en faire un outil précieux pour beaucoup de monde : c'est une source d'informations pour les écoliers, un texte d'appoint pour les étudiants, un compendium précieux pour les praticiens et les organisations civiles, un volume de référence pour les décideurs des gouvernements, des entreprises et des métiers créatifs. Il pourrait même devenir un livre de chevet pour ceux qui aiment la nature. En bref, c'est un excellent rapport de ce qui fut à l'évidence une conversation pleine de vie. C'est à coup sûr ce que la conservation mérite.
Ashok Khosla
Président de l'UICN 
L'idée de ce livre est née lors du Forum mondial de la nature qui a eu lieu en octobre 2008 à Barcelone, en Espagne, dans le cadre du quatrième Congrès mondial de la nature $(\mathrm{CMN})^{1}$. Le Forum a accueilli plus de 7000 conservationnistes engagés qui ont discuté et débattu les problèmes urgents auxquels la biodiversité fait face aujourd'hui et ceux auxquels elle doit s'attendre à l'avenir. Plus de 900 événements ont eu lieu pendant les quatre jours du Forum, et ce livre tente d'exprimer le parfum, sinon les détails, de ces discussions. Nous avons librement intégré de nombreux exemples qui furent présentés au cours des divers événements du $\mathrm{CMN}$, sans chercher à préciser de quel événement ils provenaient. Les chapitres ont une approche du sujet plutôt éclectique, ce qui reflète bien le contenu des divers événements, et ils intègrent des extraits des publications actuelles sur le sujet. Le Centre de ressources du Forum (http:// www.iucn.org/fr/congress_fr/) donne accès à toutes les informations qui ont été rendues publiques par les organisateurs et les orateurs d'événements, y compris des présentations PowerPoint, des rapports d'ateliers, des documents d'archives et des résumés.

Ce volume cherche à placer le Forum dans le cadre plus large des problèmes mondiaux de conservation. Tout en voulant capter les messages clés du CMN à Barcelone, en tant qu'éditeurs et compilateurs, nous avons aussi souhaité présenter les diverses perspectives en une synthèse cohérente qui s'inspire aussi des dernières publications en matière de conservation. Nous commençons en examinant les questions clés qui sont en jeu ; nous les abordons ensuite du point de vue de la biodiversité.
Conservation pour une ère nouvelle se veut un jalon qui présente un mode de réflexion actuel à l'intention des scientifiques, des gestionnaires et des politiciens d'aujourd'hui - tous sont confrontés à des défis liés à la biodiversité. Aucun de ces chapitres ne prétend être le mot de la fin en la matière. Au contraire, ils sont conçus pour aider à susciter et à alimenter des discussions et de futures recherches sur les différents sujets abordés. Nous espérons que ce livre va aussi pousser chacun à agir immédiatement pour relever les défis de la conservation. L'ampleur des sujets couverts dans ce volume montre aussi que la conservation du $21^{1}$ ìme siècle concerne de nombreuses parties de la société.

Nous disposons aujourd'hui de preuves plus flagrantes que jamais que la nature fait face à des menaces sans précédent, que ces menaces sont d'origine humaine, et que les solutions sont entre nos mains. L'exposé présenté ici propose quelques nouvelles orientations pour la conservation, qui vont, espérons-le, inspirer un nouveau public pour qu'il affronte ces défis et se sente poussé à l'action en faveur d'une société plus durable. À long terme, une action novatrice et participative sera notre meilleur espoir pour permettre de suivre des voies productives.

Jeffrey A. McNeely et Susan A. Mainka

1. L'UICN a toujours séparé ses Assemblées Générales, qui se concentrent sur les exigences statutaires d'une réunion de Membres, des Conférences techniques, qui se préoccupent des questions de conservation du moment. Avant la réunion de Montréal de 1996, le Conseil de l'UICN a décidé qu'il serait plus logique de les combiner en un seul événement, appelé Congrès mondial de la nature (CMN). La réunion de Barcelone était la quatrième de ce type. Les trois Congrès précédents ont eu lieu à Montréal, au Canada, en 1996, à Amman, en Jordanie, en 2000, et à Bangkok, en Thailande, en 2004. 


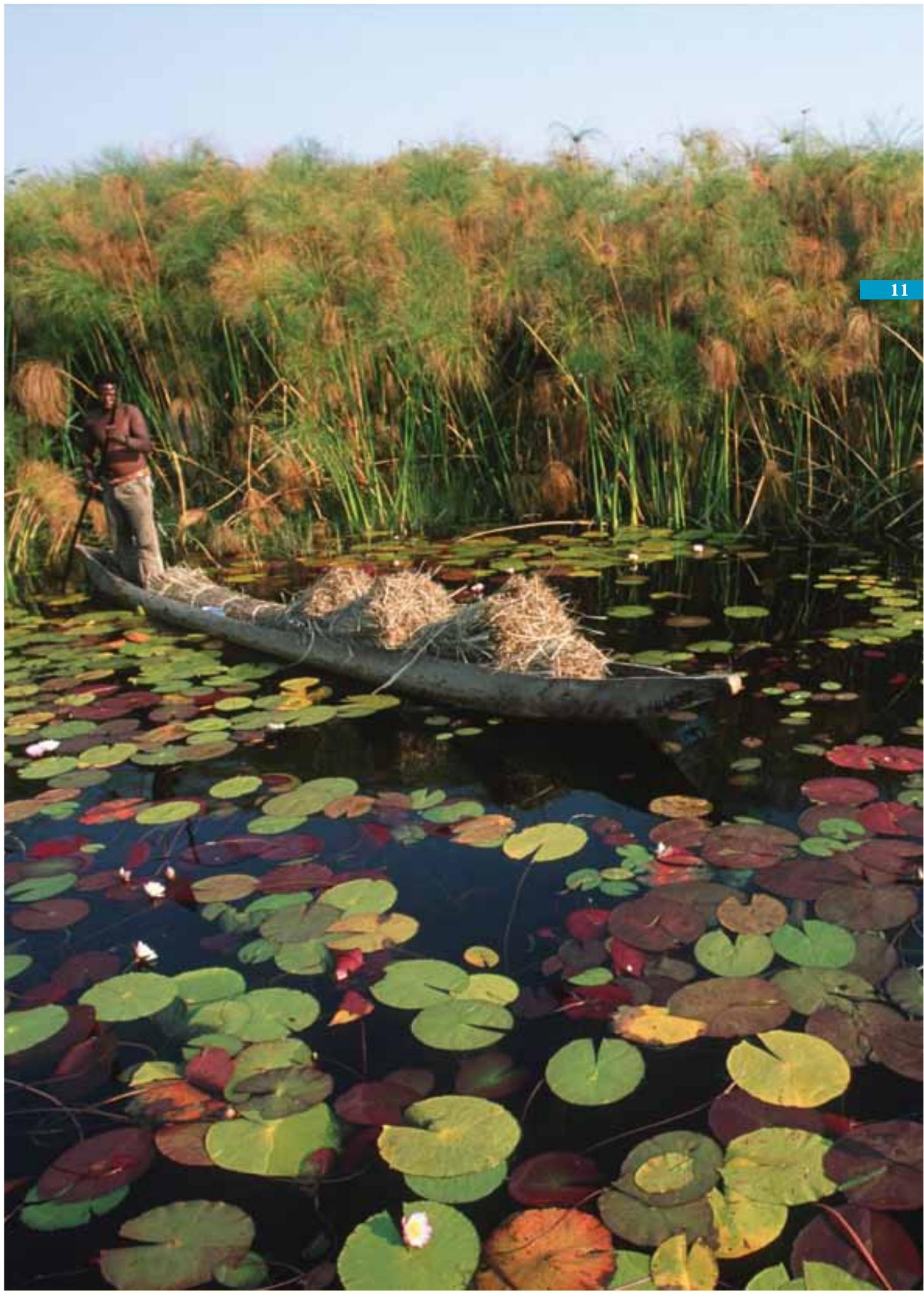




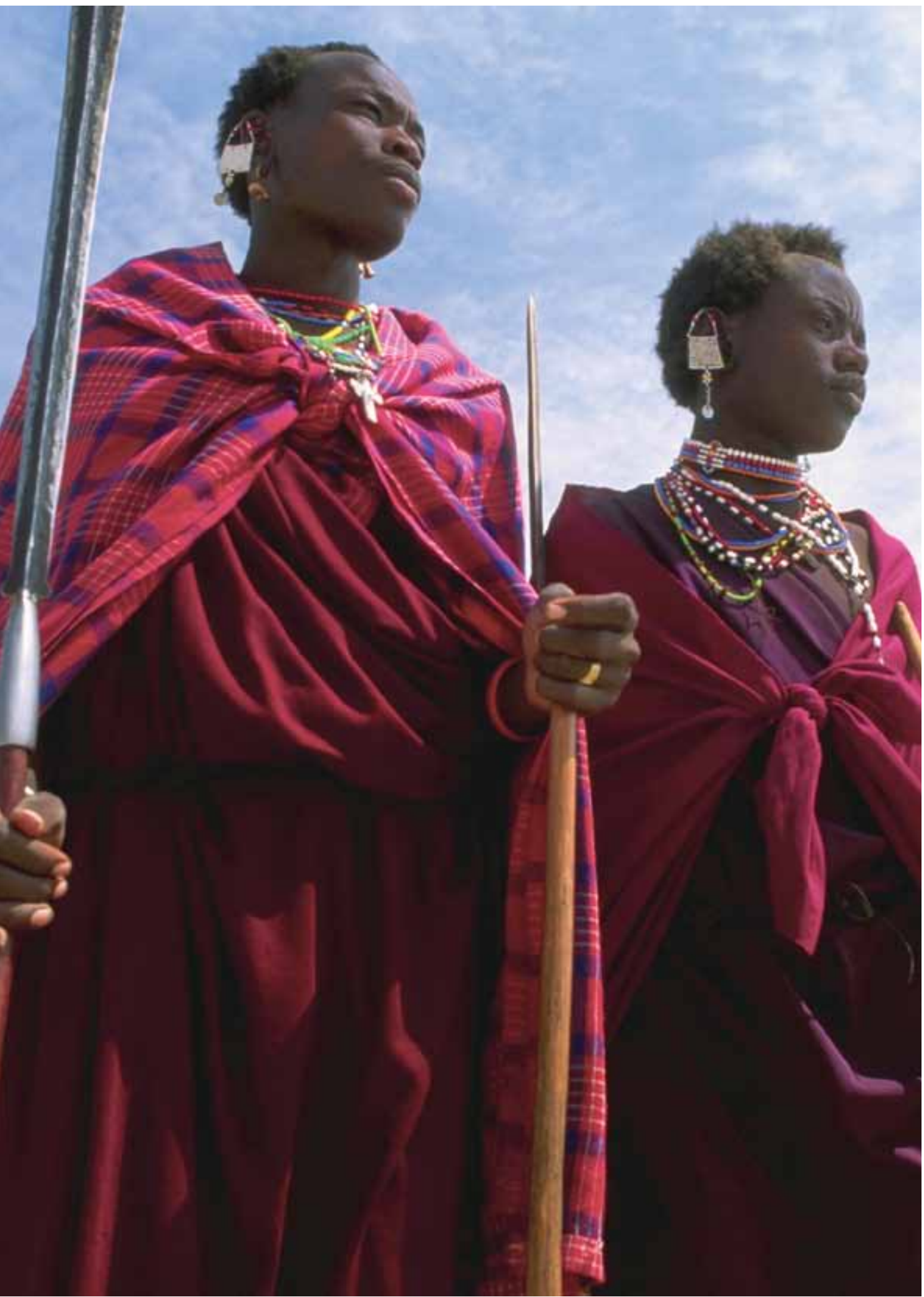




\section{Remerciements}

Ce travail a bénéficié des commentaires avisés et des contributions

substantielles des nombreuses personnes mentionnées ci-dessous. Christina Sander a fourni un service essentiel en rassemblant les divers rapports du Forum CMC et elle a aidé à la compilation d'une partie de la littérature pertinente. Deborah Murith, Stéphanie Achard et Cindy Craker ont apporté une contribution importante à la production de cette publication.

\begin{tabular}{|c|c|c|}
\hline Lorena Aguilar & Annelie Fincke & Samira Omar \\
\hline David Allen & Kristina Gjerde & Steve Osofsky \\
\hline Neville Ash & James Gordon & Gonzalo Oviedo \\
\hline Andrea Athanas & Sarah Gotheil & Georgina Peard \\
\hline Tim Badman & Marc Hockings & Sonia Pena Moreno \\
\hline Patrick Blandin & Geoffrey Howard & Jean-Yves Pirot \\
\hline Josh Bishop & David Huberman & George Rabb \\
\hline Intu Boedhihartono & Ninni Ikkala & $\begin{array}{l}\text { Pedro Rosabal } \\
\text { Gonzales }\end{array}$ \\
\hline Maria Ana Borges & $\begin{array}{l}\text { Groupe de spécialistes } \\
\text { de l'éthique de la }\end{array}$ & Christina Sander \\
\hline Guido Broekhoven & CDDE/UICN & Adel Sasvari \\
\hline Tom Brooks & Alejandro Iza & Jeffrey Sayer \\
\hline Ximena Buitron & Bill Jackson & Sara Scherr \\
\hline Giulia Carbone & Sally Jeanrenaud & David Sheppard \\
\hline Eric Chivian & Nik Lopoukhine & Kevin Smith \\
\hline Katherine Cross & Nadine McCormick & Mark Smith \\
\hline Will Darwall & Patti Moore & Jerker Tamerlander \\
\hline Jonathan Davies & Roland Melisch & Claire Warmenbol \\
\hline Saskia de Koning & Russ Mittermeier & Elizabeth Willetts \\
\hline Joao de Queiroz & Teresa Mulliken & Jean-Christophe Vié \\
\hline Nigel Dudley & James Oliver & Xie Yan \\
\hline
\end{tabular}


1. Le Forum de Barcelone :
1. Un monde divers et durable

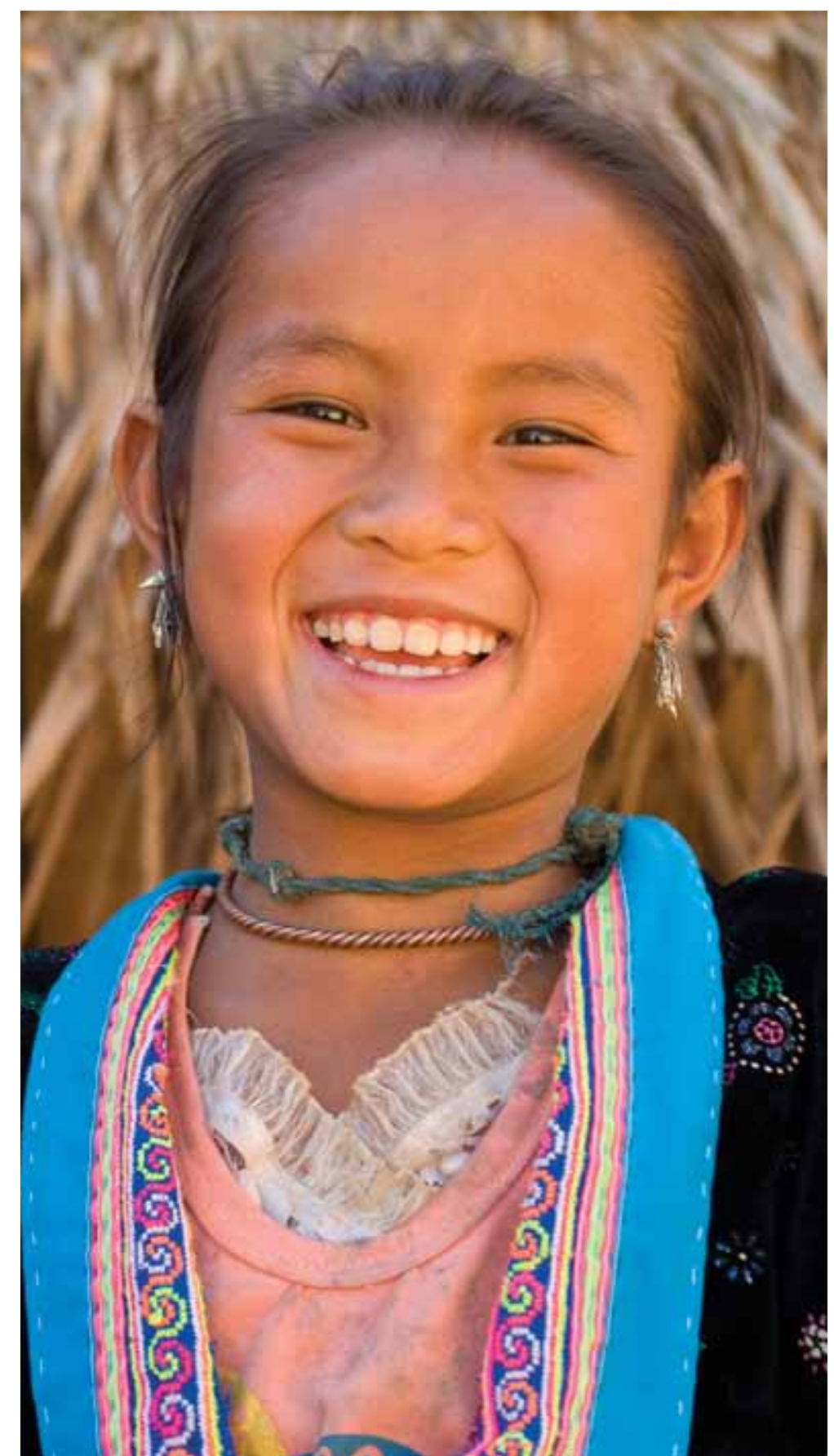




\section{La vie est résiliente. Elle se maintient depuis plus de deux millions}

d'années et elle a traversé au moins cinq crises d'extinctions massives dont la plus récente a vu la disparition des grands dinosaures, ne laissant que les oiseaux comme seuls descendants. La nature, sous l'une ou l'autre forme, va probablement survivre aux activités irréfléchies des sociétés humaines actuelles qui se fondent sur une consommation toujours croissante des ressources. Quant à savoir si ce schéma va permettre aux sociétés modernes de se perpétuer sous la forme actuelle, c'est loin d'être sûr, voire hautement improbable.

Ce livre est un relevé des défis et des stratégies discutés au Congrès mondial de la nature $(\mathrm{CMN})$ de Barcelone, en Espagne, en octobre 2008. Le thème du Congrès était Un monde divers et durable et les discussions au sein du Forum mondial de la nature se sont concentrées sur trois grands thèmes (Encadré 1.1). Ce livre ne prétend pas être complet, ce qui aurait requis que des groupes de travail passent des mois à élaborer chaque chapitre. Nous avons plutôt cherché à capter l'essence de chaque sujet, à refléter les vues de nos membres et à apporter les nouvelles perspectives issues des plus récents travaux sur le terrain, afin de catalyser les efforts de conservation de la prochaine décennie. Le Forum mondial de la nature a bénéficié de la présence dynamique de participants issus de nombreux domaines de la société, notamment d'organisations de conservation, de communautés locales et autochtones, de gouvernements (locaux et nationaux) et du monde des affaires. Voulant rester dans l'esprit de cet intérêt très divers pour la conservation, nous inclurons des actions que cette communauté de conservation élargie pourrait vouloir adopter à l'avenir.

Alors que les chapitres de ce livre reflètent toute la diversité des thèmes discutés, il sera utile de souligner dès le début quelques problèmes globaux, comme l'Objectif Biodiversité 2010, le lien entre biodiversité et développement durable, ainsi que l'atteinte des Objectifs du millénaire pour le développement (OMD) et la gestion du rapide changement démographique. 


\section{Encadré 1.1 Thèmes du Forum mondial de la nature}

\section{Sauvegarder la diversité de la vie}

La variété très riche de gènes, d'espèces et d'écosystèmes de notre planète est le fondement qui sous-tend notre diversité sociale, économique et culturelle. Depuis 60 ans, l'UICN est la force unificatrice de la conservation de la biodiversité, et ses Membres continuent à soutenir et à vouloir préserver l'importance de la nature, pour elle-même d'abord, mais aussi pour l'humanité. Malgré cette longue histoire, de nombreuses questions restent sans réponse, dans le domaine éthique (comment pouvons-nous décider qui, des hommes ou de la nature, a la priorité lorsqu'il faut faire des compromis ?) et aussi pratique (pourrons-nous nourrir neuf milliards de personnes et en même temps mettre fin à la perte de biodiversité ?). Tout en reconnaissant l'importance fondamentale de la biodiversité pour l'avenir de l'humanité, nous n'allouons pas encore suffisamment de ressources pour la conserver efficacement ; dès lors, vers qui et comment allons-nous nous tourner pour faire la différence?

\section{Un climat neuf pour le changement}

Les preuves montrent que l'environnement change plus vite qu'à aucun moment de l'histoire humaine. Au cours des 40-50 prochaines années, l'on s'attend à ce que la population mondiale atteigne 9 milliards alors qu'elle est actuellement de 6,8 milliards. Pendant ce temps, les changements du système climatique mondial s'accélèrent, et nous sommes désormais confrontés au double défi de réduire significativement et rapidement nos émissions pour éviter de dangereux changements climatiques et de nous adapter aux impacts des changements climatiques qui sont déjà en cours. Dans ce monde qui change, les gens sont de plus en plus connectés - par les moyens de communication, les transports et le commerce, mais aussi par la culture, la politique et l'environnement. Une telle " globalisation " apporte de fabuleuses opportunités mais aussi des risques. Enfin, ce besoin de croissance économique sans fin alimente des demandes toujours plus grandes d'énergie, ce qui exige que nous passions d'une économie dépendante des combustibles fossiles à des énergies mixtes qui seront plus durables.

\section{Un environnement sain pour des populations prospères}

L'utilisation durable et la conservation de la biodiversité peuvent véritablement contribuer à la réduction de la pauvreté et à la santé et au bien-être des personnes; de même, une amélioration du bien-être des personnes est une condition fondamentale pour une conservation durable. Les sociétés d'aujourd'hui sont confrontées à un défi majeur qui consiste à réconcilier le développement rural, la réduction de la pauvreté et la conservation de la biodiversité. La gestion durable de ressources naturelles telles que le poisson, les terres agricoles ou les grumes entraîne de nouveaux défis. Parmi les avancées prometteuses, citons de meilleures lois et réglementations, une planification participative à long terme et de nouveaux outils, telles les aires marines protégées. Une question clé pour le futur est «Quel genre de potentiel les aires protégées - qui ont été créées au départ pour atteindre des objectifs de conservation - ont-elles pour améliorer le bien-être des hommes et réduire la pauvreté ?» 


\section{AVANCÉES VERS L'OBJECTIF BIODIVERSITÉ 2010 ET AU-DELÀ}

De nombreux accords et conventions mondiaux concernant l'environnement ont intégré des cibles dans leurs stratégies et planifications. Parmi elles, la plus importante du point de vue de la biodiversité est l’Objectif Biodiversité 2010. L'objectif global, qui est de réduire la perte de biodiversité d'ici 2010, fut adopté dans des fora internationaux, de la Convention sur la diversité biologique (CDB) au Sommet mondial du développement durable (SMDD), encore que sous des formes quelque peu différentes (Encadré 1.2).

Le besoin de mesurer les progrès réalisés vers cette cible et au-delà a suscité le développement d'un cadre de 17 « indicateurs principaux » qui furent mentionnés pour la première fois dans le Global Diversity Outlook 2 (GBO2) (CDB, 2006) (Tableau 1.1). GBO2 résume la situation en notant que :
- La déforestation, surtout due à la conversion de forêts indigènes en plantations et en terres agricoles, continue à un rythme dramatiquement élevé.

- La tendance de quelque 3000 populations d'espèces sauvages présente un déclin continu de l'abondance moyenne des espèces de près de $40 \%$ entre 1970 et 2000 .

- Des espèces toujours plus nombreuses sont menacées d'extinction, y compris $12 \%$ des oiseaux, $21 \%$ des mammiferes et $31 \%$ des amphibiens, selon la Liste Rouge de l'UICN des espèces menacées 2008.

En 2006, reconnaissant que la science qui soustend nombre de ces indicateurs requiert encore une attention considérable, 24 organisations travaillant sur les indicateurs (dont l'UICN) ont créé le Partenariat relatif aux indicateurs de biodiversité 2010 (BIP), une initiative mondiale

\section{Encadré 1.2 L'Objectif Biodiversité 2010}

L'Objectif Biodiversité 2010 fut adopté sous plusieurs formes et fait partie de nombreux instruments de politique internationale :

- Juin 2001 - Le Sommet européen de Göteborg où plusieurs chefs d'Etat ont d'abord adopté la cible disant que «le déclin de la biodiversité doit être stoppé [dans l'UE] avec l'objectif d'atteindre cette cible en $2010 »$.

- Mai 2002 - La sixième Conférence des Parties (COP) à la Convention sur la diversité biologique (CDB) a inclus dans le Plan stratégique qu'elle a adopté une cible 2010 (" pour atteindre - cette fois - en 2010 une réduction significative du rythme actuel de perte de biodiversité au niveau mondial, régional et national, comme une contribution à l'atténuation de la pauvreté et pour le bien de toute vie sur terre $»$ ).
- Septembre 2002 - Le Sommet mondial du développement durable (SMDD) qui s'est tenu à Johannesburg a confirmé l'Objectif Biodiversité 2010 et a appelé « à l'atteinte d'ici 2010 d'une réduction significative du rythme actuel de perte de diversité biologique ».

- Mai 2003 - Les Ministres de l'Environnement et les Chefs de Délégation de 51 pays ont adopté la Résolution de Kiev sur la Biodiversité lors de la cinquième Conférence ministérielle « Environnement pour l'Europe » et ont décidé de " renforcer notre objectif de mettre fin à la perte de diversité biologique à tous les niveaux d'ici l'année 2010 ».

- Septembre 2007 - Les NU ont décidé d'adopter l'Objectif 2010 (en termes de rythme deperte) comme sous-objectif de l'Objectif 7 du millénaire pour le développement Durabilité environnementale 
D’après l'évaluation du chapitre 2 de Global Biodiversity Outlook 2. Les flèches indiquent la direction des tendances (les grosses flèches indiquent un degré de confiance élevé de la tendance, les flèches fines indiquent un faible degré de confiance, les flèches noires marquent une tendance négative pour la biodiversité, les flèches blanches indiquent une tendance positive pour la biodiversité). La qualité des données et des indicateurs est signifiée par les étoiles qui se trouvent à droite.

$\star \star \star$ bonne méthodologie par indicateurs avec des données globalement cohérentes dans le temps

$\star \star$ bon indicateur mais pas de données suivies dans le temps

$\star$ indicateur nécessitant un développement et/ou données limitées

DOMAINE FOCAL : Statut et tendances des composantes de la diversité biologique

\begin{tabular}{|c|c|c|}
\hline $\mathbf{y}$ & Tendances de l'extension de biomes, écosystèmes et habitats choisis & 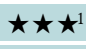 \\
\hline $\mathbf{Y}$ & Tendances de l'abondance et de la distribution d'espèces sélectionnées & $\star \star \star \star$ \\
\hline $\mathbf{y}$ & Changement du statut d'espèces menacées & $\star \star \star$ \\
\hline$\searrow$ & $\begin{array}{l}\text { Tendances de la diversité génétique d'animaux domestiques, de plantes cultivées } \\
\text { et d'espèces de poissons d'importance socio-économique majeure }\end{array}$ & $\star$ \\
\hline$\lambda$ & Couverture des aires protégées & $\star \star \star$ \\
\hline
\end{tabular}

DOMAINE FOCAL : Intégrité de l'écosystème et biens et services de l'écosystème

Indice trophique marin

\ Connectivité - fragmentation des écosystèmes

1. Qualité de l'eau des systèmes aquatiques

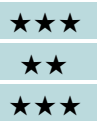

DOMAINE FOCAL : Menaces sur la biodiversité

Dépôt d'azote

Tendances des espèces exotiques envahissantes

DOMAINE FOCAL : Utilisation durable

\ Surface d'un écosystème forestier, agricole et aquaculture en gestion durable

$\nabla$ Empreinte écologique et concepts apparentés

DOMAINE FOCAL : Statut des savoirs traditionnels, des innovations, des pratiques

\Statut et tendances de la diversité linguistique et nombre de personnes pratiquant les langues autochtones $\star$ DOMAINE FOCAL : Statut de l'accès et du partage des bénéfices

? Indicateur de l'accès et du partage des bénéfices à mettre au point

DOMAINE FOCAL : Statut du transfert des ressources

\ Assistance officielle au développement (AOD) fournie, en support à la Convention

${ }^{1}$ pour les forêts : données non disponibles pour tous les biomes, écosystèmes et habitats

pour développer davantage et promouvoir

l'utilisation d'indicateurs pour le suivi cohérent et l'évaluation de la biodiversité (http://www. twentyten.net/Home/tabid/38/Default.aspx).

En se fondant sur les informations du rapport et sur celles de l'Évaluation des écosystèmes pour le millénaire (EM) (2005), le GBO2 conclut que la perte de biodiversité « est susceptible de se poursuivre dans un avenir proche, et certainement au-delà de 2010 ». Néanmoins, le GBO2 reconnaît la possibilité de réussites dans la conservation de la biodiversité et notamment: 


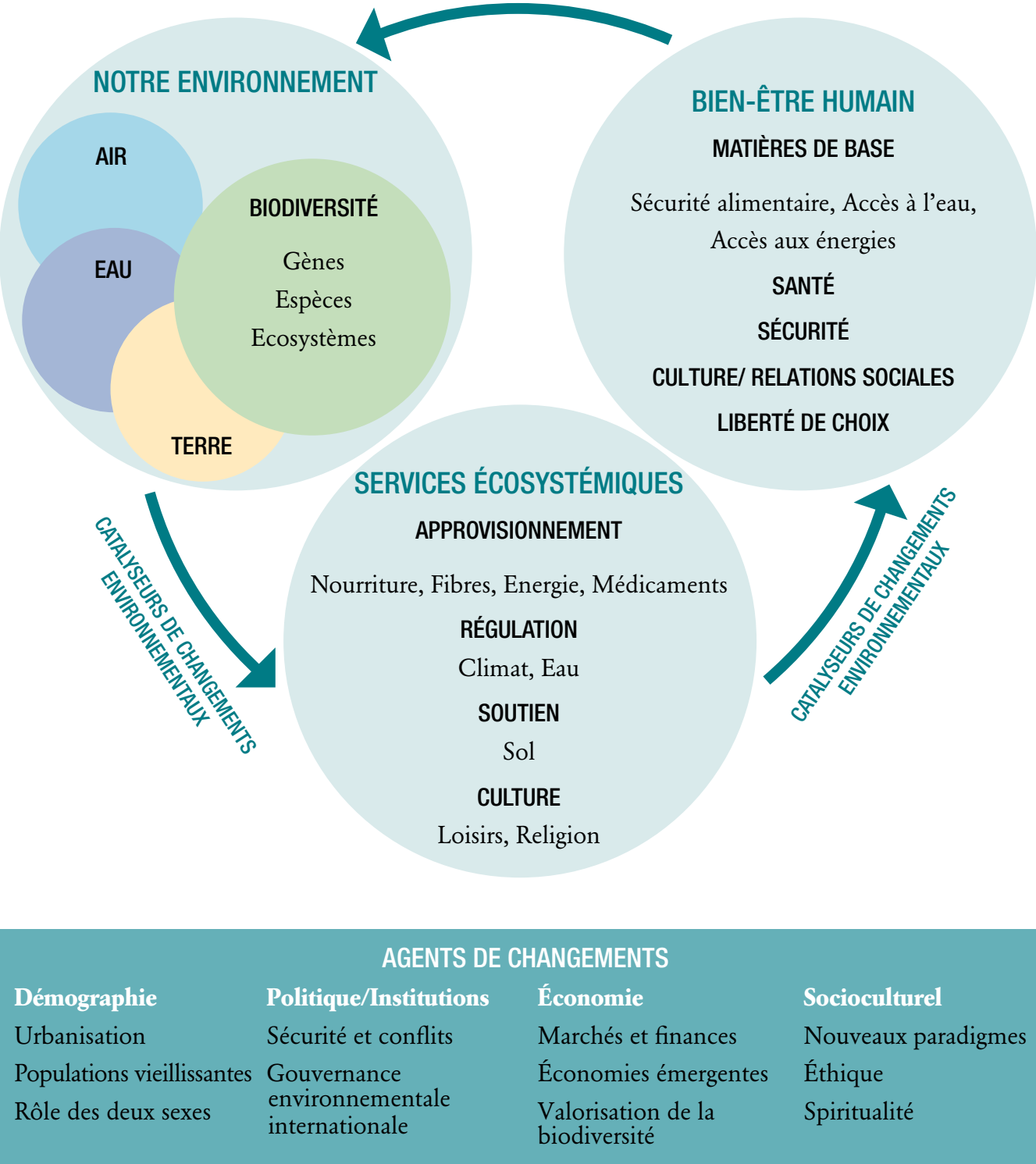

1) aux niveaux national, régional et mondial, avec des réponses appropriées, il est possible d'arriver, en 2010, à une réduction du rythme de perte de biodiversité pour certaines de ses composantes ou pour certains indicateurs, dans certaines régions ;

2) la majorité des cibles que la Convention s'est fixées dans le cadre de l'évaluation des progrès réalisés vers l'objectif 2010 sont réalisables, pour autant que les mesures nécessaires soient prises ; et

3) la plupart des outils nécessaires pour atteindre l'objectif de 2010, y compris les programmes de travail, les principes et les lignes directrices, sont déjà mis au point. 
Les efforts pour atteindre l'objectif 2010 ont été des moyens importants pour instaurer la sensibilisation, la capacité et la volonté politique en faveur de la conservation de la biodiversité. La communauté mondiale devrait s'appuyer sur cette avancée en adoptant un canevas post-2010 visionnaire, réaliste et mesurable.

\section{LIER LA BIODIVERSITÉ, LES SERVICES ÉCOSYSTÉMIQUES, LA RÉDUCTION DE LA PAUVRETÉ ET LE DÉVELOPPEMENT DURABLE}

Notre environnement, les services fournis par les écosystèmes et le bien-être des hommes sont le résultat d'une trame complexe d'interactions et de réponses. D'un point de vue pratique, quel que soit le point d'entrée dans le système que nous utilisions, la conservation des espèces, la gestion d'un écosystème ou le soutien à la fourniture de services écosystémiques, nous parlons en fin de compte d'un même impératif : soutenir le système au sein duquel nous vivons (Figure 1.1).

En 2008, la Banque mondiale a estimé à 1,4 milliard le nombre de personnes vivant dans un état d'extrême pauvreté, la majorité se trouvant en Afrique sub-saharienne et en Asie du Sud. Alors que le pourcentage de celles qui vivent dans la pauvreté a diminué ces dernières années dans la plupart des régions du monde, il est resté stable en Afrique sub-saharienne. Le Rapport mondial sur le développement humain 2008 note que les solutions pour réduire la pauvreté varient d'une région à l'autre (Banque mondiale, 2008). Pour les régions sub-sahariennes, une productivité agricole accrue est la clé de la croissance tandis qu'en Asie, la réussite consisterait à réduire l'écart sans cesse croissant entre bien-être citadin et rural.

Les conservationnistes comprennent l'importance de la nature pour elle-même. Mais ils reconnaissent aussi que la biodiversité peut jouer un rôle essentiel de support et d'amélioration des moyens de subsistance des populations.
La conservation peut contribuer à la réduction de la pauvreté, spécialement en restaurant des écosystèmes et en améliorant l'accès des pauvres aux services écosystémiques ; elle contribue ainsi à sécuriser les moyens de subsistance pour les gens qui en dépendent (Fisher et al., 2005). Mais il reste compliqué d'expliquer le lien entre la conservation de la biodiversité et la réduction de la pauvreté /le développement.

La popularisation de l'idée de services écosystémiques (Chapitre 4) par Gretchen Daly (1997) et la publication subséquente de l'Evaluation des écosystèmes pour le millénaire moins d'une décennie plus tard, en 2005, ont aidé à susciter une façon de penser qui favorise la collaboration et la coopération entre les professionnels de la conservation et du développement. Le concept de services écosystémiques souligne le rôle important de la conservation des espèces et de la gestion des écosystèmes dans notre vie quotidienne. Lorsqu'il est question de services écosystémiques, on parle des gènes, des espèces et des écosystèmes qui portent et délivrent ces services.

\section{LIER LA RÉDUCTION DE LA PAUVRETÉ AUX SERVICES ÉCOSYSTÉMIQUES}

Les liens les plus évidents entre la réduction de la pauvreté et les services écosystémiques se trouvent dans les services d'approvisionnement, qui concernent l'apport de nourriture (Chapitre 20), de médicaments (Chapitre 10), de produits forestiers (Chapitre 16) et, enfin, de revenus (Chapitre 12).

En 2008, l'Organisation des Nations Unies pour l'alimentation et l'agriculture (FAO) (FAO, 2008c, d) notait que la faim dans le monde augmente et que la répartition des personnes affamées se concentre largement sur l'Afrique sub-saharienne. Elle estimait le nombre de personnes affamées à 950 millions en 2008, une 
augmentation de plus de 80 millions par rapport à la période de base, 1990-1992. Les estimations à long terme (disponibles jusqu'en 2003-2005) montrent que certains pays sont sur la bonne voie pour atteindre l'OMD 1 qui est de réduire la faim de moitié d'ici 2015 (Tableau 1.2). Mais la période actuelle de produits alimentaires chers contrarie les progrès et elle frappe très durement les plus pauvres, les sans-terres et les foyers dirigés par des femmes.

Sous-tendant cette insécurité alimentaire, spécialement en Afrique, il y a les tendances modifiées des précipitations, qui entraînent une chute de la productivité pour les petits agriculteurs qui dépendent des pluies pour leur production. Ceci en appelle à de nouvelles approches de l'agriculture. L'éco-agriculture est un exemple d'approche de l'utilisation des terres qui intègre trois objectifs majeurs - la conservation de la biodiversité, une productivité agricole accrue et des moyens de subsistance ruraux durables (McNeely et Scherr, 2003). Il sera essentiel d'investir dans un développement agricole fondé sur l'écosystème, en suivant les lignes des approches de l'éco-agriculture et en s'adaptant aux impacts des changements climatiques pour relever le défi de la faim en Afrique rurale (Ecoagriculture partners, 2009). Des approches similaires seront nécessaires dans d'autres secteurs ; des écosystèmes ont déjà été identifiés et la technologie et les pratiques existent déjà pour pratiquer une conservation de forêts, de l'eau, de terres arides et côtières à l'échelle du paysage (voir les chapitres correspondants pour plus d'information).

Une fourniture fiable de ressources naturelles est une source d'emplois (et de revenus) pour des millions de gens de par le monde. Par exemple, dans le monde, plus d'1,3 milliard de personnes étaient engagées dans l'agriculture en 2002, et 34,5 millions de gens travaillaient dans la pêche et l'aquaculture en 2000 (www.earthtrends.
Encadré 1.3 Cinq raisons d'intégrer la conservation environnementale aux activités de développement et de réduction de la pauvreté

\section{Les pays pauvres dépendent d'avantages} environnementaux fragiles. Ces avantages, qu'ils soient privés ou sous forme d'un accès à des biens communs, constituent la principale source de revenus et de survie des pauvres.

2. Alors que la plupart des biens faits par l'homme se déprécient avec le temps, et parfois très vite, la plupart des ressources naturelles peuvent être préservées, voire augmentées par des efforts modestes si elles sont gérées correctement.

3. 17\% des années perdues ajustées pour l'incapacité (DALY) dans les pays en développement sont dues à un état médiocre de l'environnement, par rapport à $4 \%$ seulement dans les pays de l'OCDE. Le manque d'eau potable et d'assainissement correct en constitue, de loin, la cause la plus importante, comptant pour $40 \%$ des pertes de DALY induites par l'environnement dans les pays en développement ; la mauvaise qualité de l'air à l'intérieur est la seconde cause.

4. L'agriculture souffre actuellement de graves sous-investissements, ce qui entraîne la perte de biens naturels précieux qui sont des sources de revenus (ex. biodiversité, sols fertiles à cause du délavage et de la salinisation, récifs et côtes) particulièrement importantes pour réduire la pauvreté et améliorer la croissance des revenus économiques.

5. Une gestion environnementale raisonnable pourra réduire la sensibilité aux phénomènes naturels extrêmes et les impacts des changements.

Adapté de Hansen, 2007 
Tableau 1.2 Liens clés entre les Objectifs du millénaire pour le développement et l'environnement

\section{Objectifs du millénaire \\ Exemples de liens avec l'environnement pour le développement}

Objectif 1

Réduire l'extrême pauvreté et la faim
- Les stratégies en matière de subsistance et la sécurité alimentaire des pauvres dépendent souvent directement d'écosystèmes en bon état et de la diversité des services qu'ils procurent.

- Des droits précaires aux ressources environnementales, ainsi qu'un accès inadéquat aux informations, aux marchés et aux prises de décisions concernant l'environnement limitent la capacité des pauvres de protéger leur environnement et d'améliorer leurs moyens de subsistance et leur bien-être.

\section{Objectif 2}

Assurer l'éducation primaire pour tous

Objectif 3

Promouvoir l'égalité

des sexes et

l'autonomisation

des femmes
- Le temps que les enfants, et spécialement les filles, passent à aller chercher de l'eau et du bois de feu peut réduire le temps qu'ils accordent à l'étude.

- Les revenus supplémentaires tirés d'une gestion durable des ressources naturelles peuvent être consacrés à l'éducation.

- Le temps que les femmes consacrent à aller chercher de l'eau et du bois de feu réduit la possibilité d'activités rémunératrices.

- Les femmes rurales pauvres dépendent souvent beaucoup des ressources naturelles, mais les inégalités et l'absence de droits établis limitent leur accès aux prises de décisions et aux ressources.

\section{Objectif 4}

Réduire la mortalité infantile

\section{Objectif 5}

Améliorer la santé maternelle

Objectif 6

Combattre le VIH/

SIDA, le paludisme et d'autres maladies

\section{Objectif 7}

Assurer un environnement durable
- Une meilleure gestion des bassins versants locaux peut réduire la mortalité infantile causée par les maladies liées à l'eau.

\section{Objectif 8}

Mettre en place un partenariat mondial pour le développement
- La pollution de l'air à l'intérieur des maisons et le fait de transporter de lourdes charges jusqu'à un stade avancé de la grossesse mettent la santé des femmes en danger avant l'accouchement.

- Les facteurs de risques environnementaux peuvent parfois être impliqués dans $20 \%$ du total des maladies dans les pays en développement.

- Des mesures préventives en faveur de la santé environnementale sont aussi importantes, et parfois plus rentables, que les traitements sanitaires.

- Tous les autres objectifs sont liés à la durabilité environnementale, souvent de façon très directe (comme c'est décrit ailleurs dans ce livre).

- L'interaction complexe entre bien-être humain, services écosystémiques et biodiversité exige une approche intégrée qui comprend des partenariats entre la société civile, le secteur privé et le gouvernement. 
org). Sur une échelle plus petite, les ressources naturelles locales représentent une proportion importante des revenus des ménages, en plus des simples moyens de subsistance. Au niveau national, les ressources naturelles comptent aussi beaucoup : en Tanzanie, l'utilisation de l'environnement et des ressources naturelles représente $66 \%$ du produit national brut (PNUE, 2008a).

L'importance des ressources naturelles dans les économies nationales, spécialement dans les pays en développement, est une motivation importante pour garantir qu'une sage gestion de l'environnement fasse intégralement partie des stratégies nationales de développement et de croissance. Les gouvernements des pays en développement et les organismes d'aide au développement reconnaissent déjà le rôle crucial qu'une gestion environnementale raisonnable va jouer dans la réussite de la réduction de la pauvreté (Hansen, 2007) (Encadré 1.3).

Le défi actuel de l'aide au développement est de voir comment intégrer au mieux l'environnement dans le processus visant à améliorer le bien-être humain. L'intégration environnementale doit se faire tant au niveau de la planification que lorsque les activités sont entreprises. Bojo et al. (2004) rapportaient que le taux d'intégration de l'environnement dans 53 articles révisés concernant la stratégie de réduction de la pauvreté était très variable, mais que le niveau global s'améliorait par rapport à des études antérieures. Comme dans tout programme de gestion de l'environnement, les efforts en vue de la réduction de la pauvreté doivent inclure une approche adaptative de la gestion pour garantir une réponse adéquate aux changements environnementaux et sociaux.

\section{DÉBAT CONSERVATION VS RÉDUCTION}

\section{DE LA PAUVRETÉ}

La communauté de la conservation discute activement pour savoir si et dans quelle mesure les conservationnistes peuvent réellement contribuer à des efforts visant au développement mondial et à la réduction de la pauvreté. Intégrer dans des projets de conservation les besoins de communautés locales qui se font de plus en plus entendre est un défi supplémentaire pour ceux qui travaillent sur le terrain. Roe (2008) a résumé cette évolution du débat conservation/réduction de la pauvreté en notant qu'avec les années, les communautés s'occupant de conservation et de réduction de la pauvreté avaient alternativement convergé et divergé. Elle a découvert qu'il n’y a en fait que peu de personnes qui vivent dans certaines des régions qui ont le plus besoin de conservation, mais que ces personnes sont souvent très pauvres et souffrent beaucoup si on leur refuse l'accès aux ressources. De plus, ces personnes vivent souvent là depuis des générations, et le fait que cette région ait une valeur pour la conservation indique bien que leurs activités ne sont pas incompatibles avec la conservation. D'autre part, les pressions du développement moderne peuvent balayer les pratiques traditionnelles de conservation et de gestion des ressources, entraînant la perte de biodiversité et de services environnementaux. La relation entre conservation et développement dans des régions qui abritent une biodiversité de valeur nationale ou mondiale exceptionnelle est très complexe et elle exige toujours des solutions spécifiques au site concerné.

\section{LES OBJECTIFS DU MILLÉNAIRE POUR LE DÉVELOPPEMENT ET L'ENVIRONNEMENT}

En 2000, la Déclaration du millénaire a pris acte de l'engagement des membres des Nations Unies d'éradiquer l'extrême pauvreté et la faim et de construire un monde plus sûr et pacifique, propice au développement humain. Des cibles ambitieuses furent inscrites dans les Objectifs du millénaire pour le développement (OMD), et l'on mit au point des indicateurs pour en évaluer les progrès. L'inscription des OMD et 
des cibles annexes sur une liste peut sembler impliquer qu'ils constitueraient une sorte de liste de contrôle de points qui pourraient être réalisés un par un. Pourtant, il est de loin préférable de les voir comme un ensemble où les progrès pour réaliser un OMD ou une cible dépendraient aussi de la réalisation des autres. Si l'OMD 7 est le seul objectif qui vise directement l'environnement, la réalisation de chacun des objectifs exige pourtant le support d'un environnement en bon état de fonctionnement. D'autre part, la réalisation des autres OMD aidera à celle de l'OMD 7 (Tableau 1.2). Alors que les liens entre l'environnement et le bien-être humain sont de mieux en mieux articulés, les menaces qui pèsent sur les deux le sont aussi. Nous notons particulièrement que les changements climatiques, les espèces exotiques envahissantes et l'utilisation non durable des ressources émergent comme des domaines clés qui doivent être traités dans la planification non seulement de la conservation mais aussi de la réduction de la pauvreté.

Un examen des progrès accomplis vers les OMD, essentiellement réalisés à mi-chemin entre l'année de la définition des cibles et le délai fixé pour atteindre les objectifs proprement dits, a révélé que, si quelques succès ont bien été enregistrés, il reste beaucoup à faire (NU, 2008). Le rapport a identifié de nombreux domaines dans lesquels « un plus grand effort » était attendu, y compris :

\section{FIGURE 1.2 TENDANCES DE LA POPULATION HUMAINE}

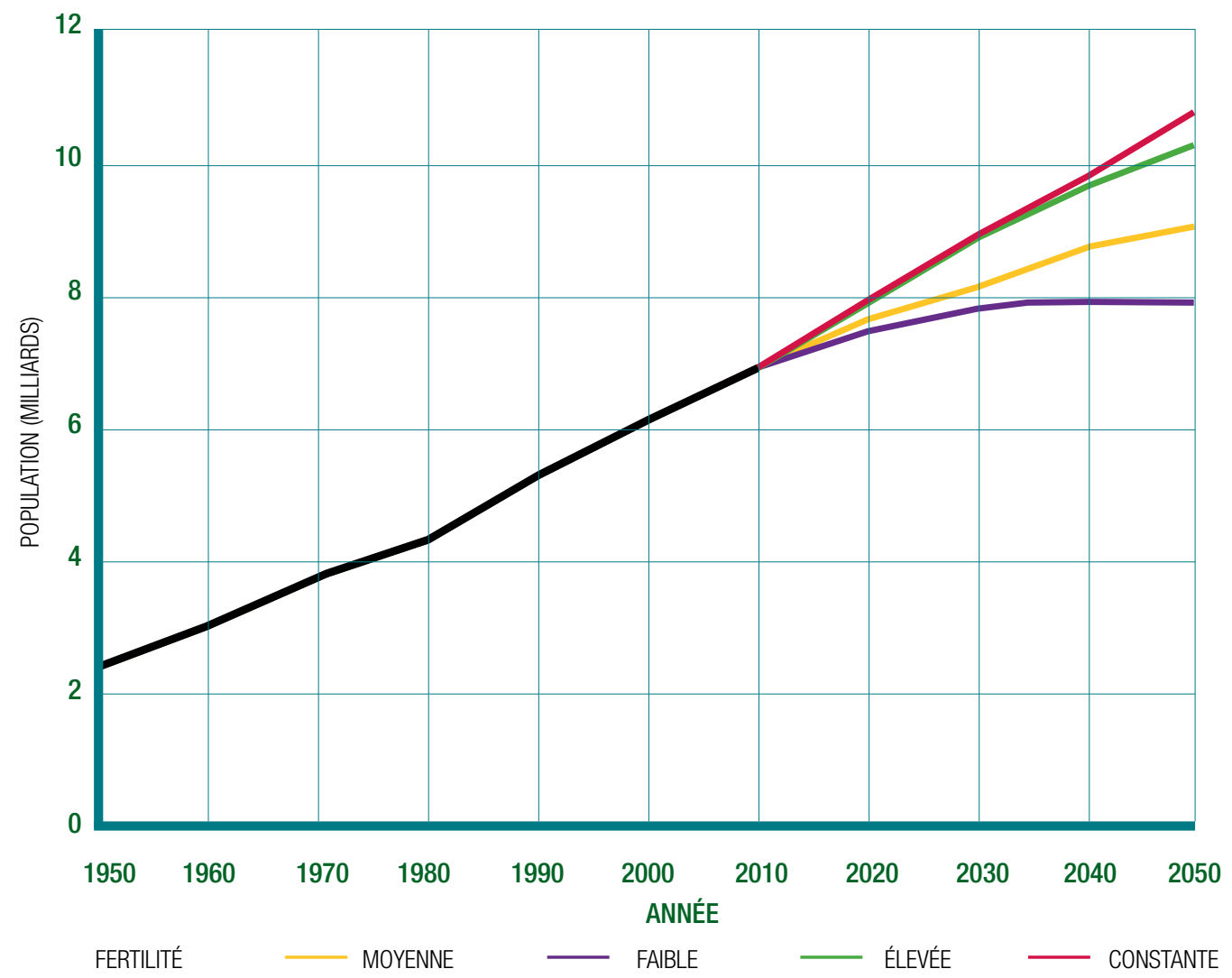




\section{Encadré 1.4 Projections clés pour la population mondiale}

\section{Nombre total}

- En supposant que la fertilité continue à décliner, on s'attend à ce que la population mondiale atteigne 9,1 milliards en 2050 et qu'à cette date, elle croisse encore de 33 millions par an environ.

- La croissance future de la population dépend fortement de la voie que prendra la fertilité à l'avenir, mais la croissance de la population jusqu'en 2050 est inévitable même si le déclin de la fertilité s'accélère.

- La croissance de la population des 49 pays les moins développés est toujours la plus rapide $\mathrm{du}$ monde, avec 2,3\% par an.

- Entre 2010 et 2050, neuf pays devraient compter pour la moitié de l'augmentation attendue de la population mondiale : l'Inde, le Pakistan, le Nigeria, l'Ethiopie, les États-Unis, la République Démocratique du Congo, la République Unie de Tanzanie, la Chine et le Bangladesh, classés ici selon l'importance de leur contribution à la croissance de la population mondiale.

\section{Démographie}

- Globalement, le nombre de personnes âgées de 60 ans et plus devrait presque tripler, passant de 739 millions en 2009 à 2 milliards en 2050.

- Globalement, l'espérance de vie à la naissance devrait passer de 68 ans en 2005-2010 à 76 ans en 2045-2050.

- En termes de moyennes annuelles nettes, les principaux pays d'accueil des migrants internationaux durant la période 2010-2050 devraient être les États-Unis, le Canada, le Royaume-Uni, l'Espagne, l'Italie, l'Allemagne, l'Australie et la France. Les principaux pays d'exportation nette devraient être le Mexique, la Chine, l'Inde, les Philippines, le Pakistan, l’Indonésie et le Bangladesh.

Source : UN-DAES, 2009

- La proportion de gens qui vivent avec moins d'1 \$US par jour en Afrique sub-saharienne est peu susceptible d'être réduite par la cible qui est de $50 \%$;

- On estime que près d'un quart de tous les enfants des pays en développement sont trop maigres et risquent de voir leur avenir compromis par les effets à long terme de la malnutrition ;

- Des 113 pays qui n'ont pas réussi à atteindre a parité des sexes pour les inscriptions dans l'enseignement primaire et secondaire en 2005 , seuls 18 sont susceptibles d'atteindre cet objectif en 2015 ;

- Près des deux-tiers des femmes employées dans le monde en développement ont des emplois fragiles : elles travaillent pour leur compte propre ou, impayées, pour leur famille ;
- Dans un tiers des pays en développement, les femmes représentent moins de 10\% des parlementaires ;

- Plus de 500.000 futures mères de pays en développement meurent chaque année lors de l'accouchement ou suite à des complications de la grossesse ;

- Quelque 2,5 milliards de gens, soit près de la moitié de la population des pays en développement, vivent sans installations sanitaires correctes ;

- Plus d'un tiers de la population urbaine croissante des pays en développement vit dans des bidonvilles ;

- Les émissions de dioxyde de carbone ont continué à augmenter, malgré le programme international établi pour aborder ce problème ; 
- Les dépenses de l'aide internationale pour les pays en développement ont diminué pour la deuxième année consécutive en 2007, et risquent d'être inférieures aux promesses faites en 2005 ; et

- Les négociations en matière de commerce international ont des années de retard, et leurs résultats, quels qu'ils soient, seront probablement très inférieurs aux grands espoirs initiaux de résultats orientés vers le développement.

Étant donné le rôle important de l'environnement pour atteindre tous les OMD, il est évident qu'il faut apporter une plus grande attention à l'environnement pour les atteindre.

\section{TENIR COMPTE DES CHANGEMENTS : DÉMOGRAPHIE ET CONSERVATION}

Outre l'Objectif 2010 et les discussions au sujet du lien avec le développement durable, le changement fut un fil rouge commun à de nombreuses discussions de Barcelone. Les changements climatiques, technologiques et démographiques affectent tout ce que nous faisons pour la conservation de la biodiversité. Alors que le climat (Chapitre 5) et la technologie (Chapitre 13) font l'objet de chapitres spécifiques, la question de la démographie humaine vaut la peine d'être explorée dès le début dans la mesure où elle influence tant d'autres domaines.

La population humaine a quadruplé au cours du $20^{\text {ème }}$ siècle, passant de 1,5 milliard en 1900 à près de 6,8 milliards en 2009 (NU-DAES, 2009 - Figure 1.2). Cette croissance explosive de la population a atteint un sommet avec un taux de croissance de 2,1\% à la fin des années 1960, le processus démographique le plus significatif depuis le début de la révolution industrielle. Depuis lors, le taux de croissance de la population a chuté de manière spectaculaire et, contrairement aux siècles passés, quand les populations étaient touchées par des conflits majeurs ou des épidémies, la chute observée dans le monde d'aujourd'hui est liée à des choix délibérés, dans le but de limiter le nombre de naissances (Cohen, 2005).

Mais la croissance de la population seule ne suffit pas à raconter toute l'histoire. Comme le montrent les résultats de l'UN-DAES (Encadré 1.4), la proportion de personnes âgées augmente dans certains pays qui, dès 2005, comptent plus de personnes âgées d'au moins 60 ans que d'enfants de zéro à quatre ans. Cette évolution sera surtout évidente dans le monde développé où, d'ici 2050, un tiers de la population aura plus de 60 ans, comparé à 20\% seulement dans le monde en développement (Cohen, 2005). Cependant, comme ce dernier compte tellement plus de personnes, c'est encore lui qui comprendra près de $80 \%$ de la population totale des personnes de 60 ans et plus.

D'autres modifications importantes comprennent le fait que, en 2007 par exemple, plus de gens vivaient dans les villes qu'en milieu rural et que, dès 2009, la majorité des gens dans le monde se classaient parmi les "revenus moyens", ce qui signifie un nouveau pouvoir d'achat, et un impact subséquent sur la consommation accrue des ressources naturelles. Le nombre de villes qui comptent au moins un million d'habitants était de 76 en 1950, 522 en 1975, 1122 en 2000, et il devrait dépasser 1600 en 2015. En utilisant les projections de population actuelles pour 2050, on peut dire que la plus grande partie de la croissance future de la population se fera dans des villes, et que les pays pauvres auront à « construire l'équivalent d'une ville d'un million de personnes chaque semaine pendant les 45 prochaines années» (Cohen, 2005).

Un nouveau défi démographique concerne l'émergence de "migrants environnementaux ", particulièrement suite aux changements climatiques. Les populations qui vivent dans des états insulaires de très faible altitude, 
comme les Maldives et Tuvalu, ou sur des zones côtières fragiles, comme certaines parties du Bangladesh ou de la Floride, vont soulever des défis environnementaux mais aussi sociaux, économiques et sécuritaires.

Une autre prospective en matière de population est liée au nombre de ménages par rapport au nombre de gens. Liu et al. (2003) rapportaient que, même si les chiffres de population restaient stables ou baissaient, si le nombre de foyers augmente, la demande de ressources naturelles va aussi augmenter. Ils rapportent que la croissance de la population entre 1985 et 2000 dans des pays qui abritent des hauts-lieux de la biodiversité fut dépassée par la croissance du nombre de ménages, parce que la taille moyenne des ménages diminue (et diminue plus rapidement que dans les pays qui n'ont pas ces hauts-lieux), ce qui pose de sérieux défis pour la gestion des ressources naturelles et la conservation de la biodiversité.

Répondre aux besoins de ces populations en changement, au nombre croissant de personnes âgées et à l'extrême concentration des zones urbaines, aura inévitablement des impacts sur l'environnement. Les zones urbaines de plus en plus peuplées devront s'étendre - souvent vers d'importantes terres arables voisines, ce qui limitera la productivité de ces terres. Les changements démographiques signifieront aussi une augmentation des dépenses publiques dans les secteurs des soins de santé et du soutien aux familles, éventuellement compensée par la réduction des investissements dans d'autres domaines publics, telle la gestion de l'environnement.

Comme ce livre explore les nombreux défis auxquels la conservation fait face aujourd'hui, il ne sera pas inutile de garder à l'esprit les questions sous-jacentes discutées plus haut, et la façon dont elles affecteront les choix et les actions des décennies à venir.

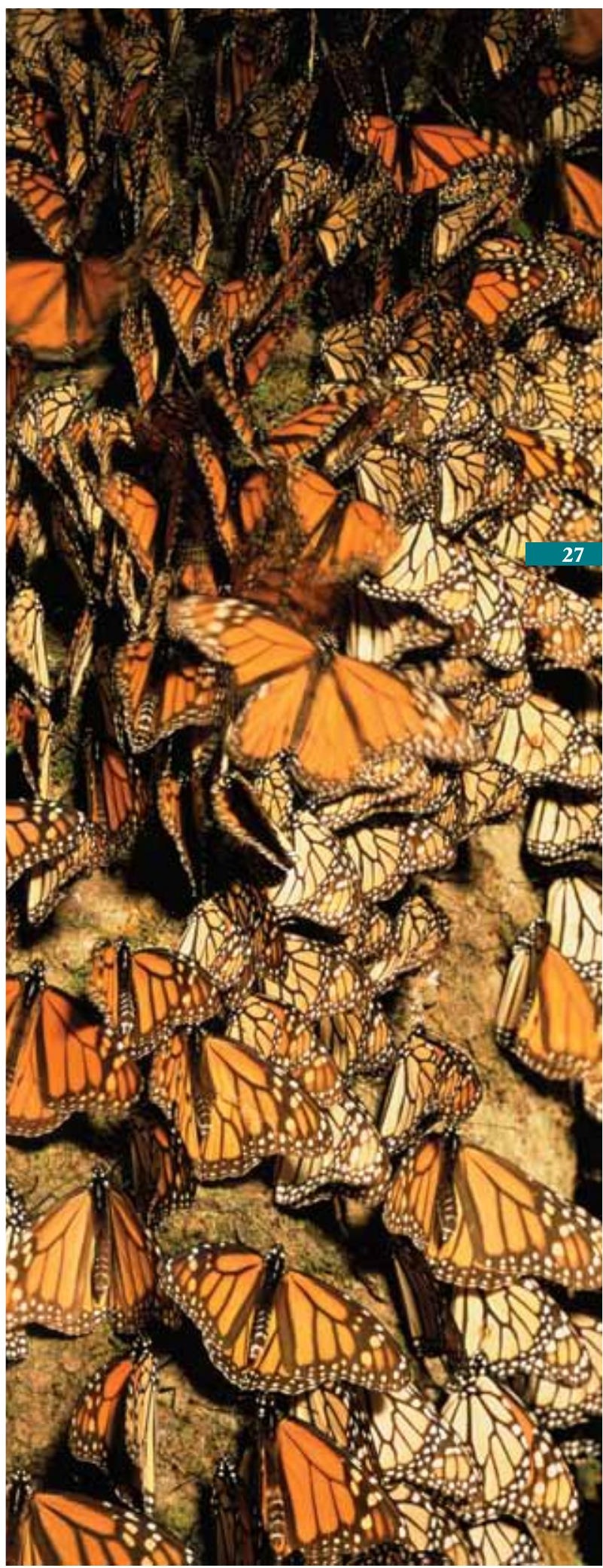




\section{Léthique de la conservation du $21^{\text {ème }}$ siècle}
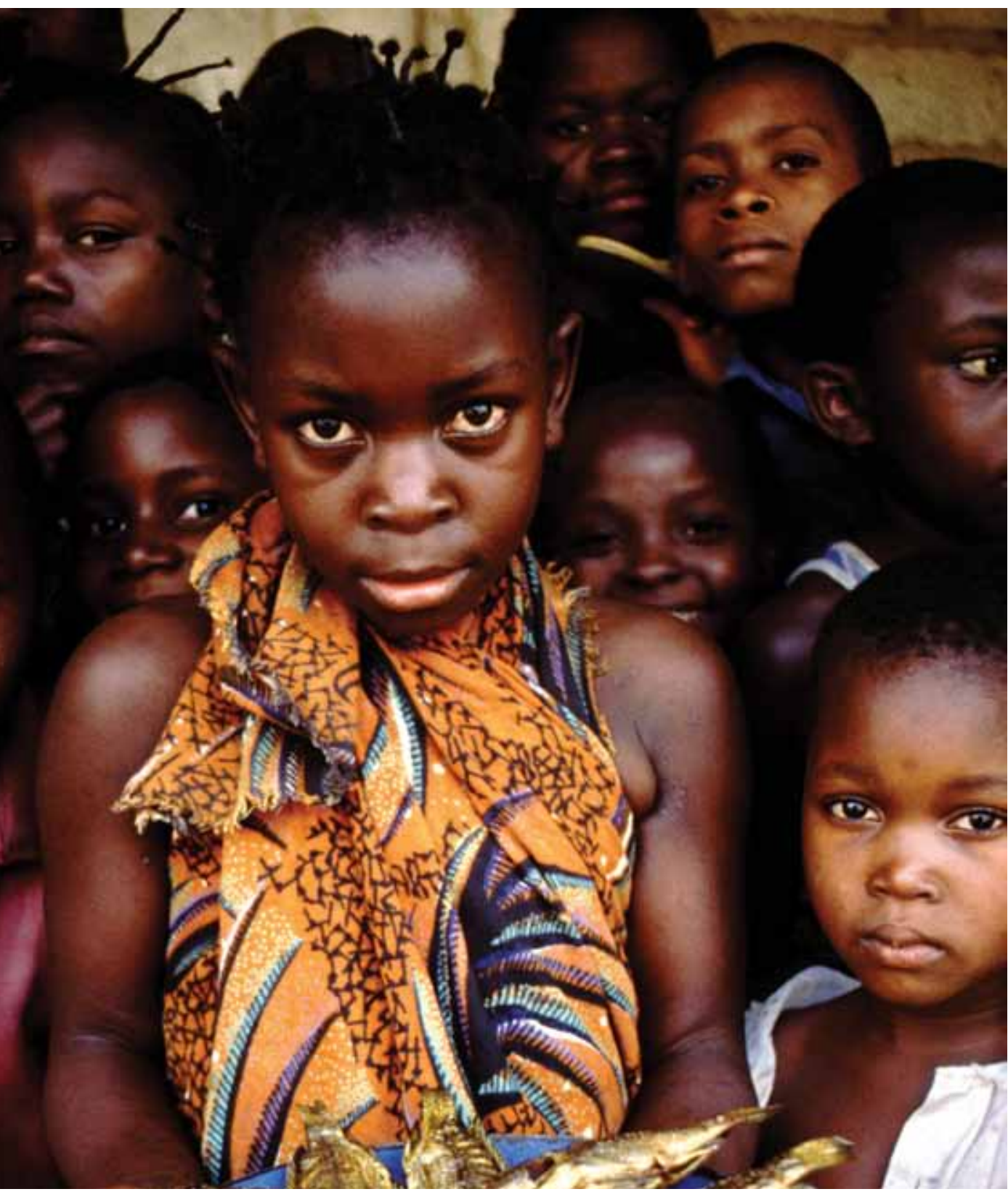
L'éthique, ce sont les principes généraux qui orientent les prises de décisions humaines, sous l'influence de facteurs culturels, des religions, de l'économie, des connaissances et des sciences. L'éthique touche à des valeurs collectives constituées de réponses individuelles et personnelles et elle oriente les décisions au sujet de que nous pensons que nous devrions faire et de la façon dont nous pensons que nous devrions agir. Comme la nature, l'éthique peut être très diverse. S'inspirant du travail du Groupe de travail inter-commissions sur l'Éthique en conservation, I'UICN cherche à proposer certains principes généraux qui pourraient être adaptés à une large gamme d'applications spécifiques en conservation.

La conservation, par essence, reflète les valeurs spécifiques que les individus et les sociétés attribuent à la nature et aux relations hommes-nature. La condition humaine se définit par les besoins et les réponses individuelles et collectives d'ordre physique, biologique, intellectuel et spirituel. Des cadres de référence éthiques alternatifs peuvent clarifier les systèmes de valeurs qui soutiennent les décisions prises en matière de gestion des ressources.
L'éthique de la conservation est enchâssée dans les religions depuis des siècles, et le lien entre nature et spiritualité est évident. Des voix du milieu de la conservation ont aussi débattu des liens entre religion et conservation, tout particulièrement dans une série d'articles de Conservation Biology, en 2005 (Orr, 2005 ; Stuart et al., 2005).

L'éthique est nécessaire pour susciter des changements, documenter les lois, les politiques et la recherche. L'UICN a fait des contributions cruciales à l'amélioration de l'appréhension des valeurs et des principes fondamentaux de la conservation de la nature, y compris grâce à la Stratégie mondiale de la conservation (1980), à la Charte mondiale de la nature (1982), à Caring for the Earth (1991) et à la Charte de la terre (1994).

En 1972, la Déclaration de Stockholm affirmait que « Nous avons atteint un point de l'histoire où nous devons moduler nos actions dans le monde entier en portant une attention plus prudente à leurs conséquences environnementales. Par ignorance ou par indifférence, nous pouvons causer des dommages massifs et irréversibles à l'environnement terrestre dont dépendent notre vie et notre bienêtre. Inversement, par de meilleures connaissances et des actions plus réfléchies, nous pourrons obtenir, pour nous et pour nos descendants, une vie meilleure dans un environnement plus respectueux des besoins et des espoirs humains. »

Plus tard, la Charte mondiale de la nature de 1982 déclarait que chaque forme de vie est unique, qu'elle mérite le respect quelle que soit sa valeur pour les gens et que, pour accorder à d'autres 
Toutes les

espèces et tous les

systèmes de la nature

méritent d'être respectés

quelle que soit leur utilité

pour l'humanité. 
organismes la même reconnaissance, les gens doivent se laisser guider par un code d'action moral. Les hommes peuvent changer la nature et épuiser les ressources naturelles par leurs actions ou par leurs conséquences et ils doivent donc reconnaître qu'il est urgent de préserver la stabilité et la qualité de la nature et de conserver les ressources naturelles.

Sauver la planète définit la position éthique de l'UICN comme « respectant et se préoccupant de la communauté du vivant ». Depuis lors, le monde a rencontré des défis de plus en plus significatifs nécessitant des actions collectives à l'échelle mondiale, concernant des préoccupations environnementales qui ne peuvent être résolues que par une coopération internationale. $\mathrm{La}$ responsabilité de l'homme dans la continuité de la vie est plus grande que jamais.

Comment utiliser les connaissances et comment changer les comportements en réponse à ces connaissances reste un challenge pour les conservationnistes. Les écosystèmes comme les sociétés ont profondément changé ces dernières années. Confrontée à des défis mondiaux tels que les changements climatiques, les espèces envahissantes, la perte de la biodiversité et la gouvernance en haute mer, entre autres, l'éthique est invoquée pour motiver les changements indispensables pour résoudre ces problèmes, face, souvent, à de puissantes pressions visant à accélérer la consommation. Sauver la planète insiste sur les arguments éthiques pour appuyer le raisonnement économique ou social afin d'encourager les pratiques de conservation. L'éthique ici semble fournir la base nécessaire pour mobiliser aussi bien l'action collective que la responsabilité individuelle.

\section{ACTION COLLECTIVE}

L'éthique appliquée à la conservation a évolué très vite depuis la Déclaration de Stockholm de 1972. Néanmoins, il reste toujours plus efficace de défendre les valeurs de la nature d'un point de vue social ou économique que d'un point de vue éthique. Les conservationnistes trouvent difficile de convaincre les gens d'adopter une " éthique de la conservation " ou une "bioéthique " comme raison suffisante pour changer leur comportement. Une partie de cette difficulté réside dans la définition de la nature précise de cette éthique et, spécifiquement, dans la façon de donner une valeur à l'environnement. De nombreux conservationnistes ont accepté de donner une valeur à une action de conservation, en raison de la situation éthique de la nature en elle-même. D'autres invoquent des arguments éthiques pour défendre une approche plus pragmatique ; ils se concentrent sur les avantages de la conservation pour les gens, grâce aux services écosystémiques, et reconnaissent que les pauvres sont souvent les premiers à souffrir d'une perte de biodiversité. Comme l'écrit Meffe (2005), « la conservation biologique implique d'avoir des connaissances écologiques et de prendre des décisions quant aux valeurs".

Le principe de Sauver la planète selon lequel "Toutes les espèces et tous les systèmes de la nature méritent d'être respectés quelle que soit leur utilité pour l'humanité » a été accepté par tous les gouvernements signataires de la Convention sur la diversité biologique (CDB) qui reconnaît dans son préambule « la valeur intrinsèque de la diversité biologique et des valeurs écologiques, génétiques, sociales, économiques, scientifiques, éducatives, culturelles, récréatives et esthétiques de la diversité biologique et de ses composantes".

A une époque où l'on parle de services écosystémiques et d'un marché pour ces services, le cadre de référence pour l'éthique s'est étendu à l'échelle mondiale. Sauver la planète en appelle à des « codes de conduite pratique qui mettent en œuvre l'éthique mondiale dans le contexte culturel de chaque société ". Lorsque l'on examine cette évolution, le Protocole de Montréal 
de 1987 au sujet de l'ozone apparait comme une étape importante dans la mesure où il est l'un des premiers traités internationaux contraignants concernant l'environnement mondial, même s'il était aussi d'un intérêt non négligeable pour les hommes. La question de la diminution de la couche d'ozone était un problème collectif qui fut résolu par un accord multilatéral. Le Protocole a montré qu'un mouvement environnemental international pouvait résoudre un problème collectif. A Montréal, les pays ont accepté de se soucier de la terre en sauvant la couche d'ozone, et en réalité leur peau, des rayons UV-B.

Quatre ans après

\section{Montréal, Sauver la}

planète a demandé que la conservation fasse un bond en avant, en déclarant que la nature « devait être préservée pour elle-même ». L'idée était radicalement nouvelle parce qu'elle passait d'une conception anthropocentrique, avec l'homme au centre, à une perspective plus holistique qui plaçait l'homme au sein de son environnement. Le Protocole de Montréal a montré que l'on pouvait entreprendre quelque chose pour préserver l'atmosphère, pour la santé de la planète. Sauver la planète a demandé que les gens élargissent leur motivation à agir afin de préserver plus largement la nature.

Un an plus tard, la Convention sur la diversité biologique était adoptée au Sommet de la Terre à Rio de Janeiro et elle est entrée en application en 1993. Ses objectifs prennent des positions éthiques en appelant à « la conservation de la biodiversité, à l'utilisation durable de ses composantes et au partage juste et équitable des bénéfices provenant de l'utilisation des ressources génétiques ».

Depuis Sauver la planète, le mouvement en faveur d'une convention globale entre les individus et la nature s'est amplifié. L'idée d'une Charte de la Terre fut lancée en 1992 au Sommet de la Terre. En 1995, l'initiative Charte de la Terre a développé le principe selon lequel l'éthique est essentielle pour une "société mondiale juste, durable et pacifique au $21^{\text {ème }}$ siècle ", s'inspirant de "valeurs mondiales partagées». La Charte de la Terre couvre des problèmes mondiaux qui réunissent toute l'humanité et la lient à l'environnement. Ses thèmes incluent l'éthique mondiale et celle du développement, la démocratie, l'écologie et la religion, les changements climatiques, les biotechnologies, la santé publique, l'intégrité écologique, les droits humains environnementaux, les animaux et l'éthique, et l'éducation.

Pour traiter de questions telles que la biodiversité, le développement durable et les changements climatiques, l'éthique de la conservation est devenue essentielle pour pouvoir mobiliser les particuliers. Au cours des dernières décennies, un mode de vie durable est devenu un impératif éthique, essentiel pour gérer l'interdépendance mondiale. Cette idée de responsabilité personnelle, ressentie partout dans le monde, est révolutionnaire mais elle est essentielle si l’on veut relever les défis mondiaux lancés à l'environnement.

\section{LE RÔLE DE CHAQUE INDIVIDU}

L'éthique personnelle et l'environnement concernent désormais toute la société. L'éthique aide à définir un nouveau contrat social entre les hommes et l'environnement. Les questions environnementales que pose l'éthique sont les suivantes : comment voulons-nous vivre sur cette 
terre ? Quelle sorte de monde voulons-nous? L'avenir et la portée du changement dépendront des réponses à ces questions et de la façon dont nous convertirons ces réponses en actes.

Transformer les nombreuses réponses à ces questions en actes cohérents et productifs n'est pas chose facile. Comme le disent Meadows et al. (1972), « il n'est pas possible d'évaluer l'avenir à long terme de l'un de ces niveaux [population, capital, alimentation, ressources non renouvelables et pollution] sans prendre en compte tous les autres. Et pourtant ce système relativement simple a une structure tellement compliquée que l'on ne peut pas, intuitivement, comprendre comment il va se comporter à l'avenir ». Meadows suggère d'envisager d'éventuelles réactions en chaîne positives, comme une croissance de la population allant de pair avec une croissance industrielle, et des réactions en chaîne négatives, comme la pollution, qui devient plus forte lorsque la croissance approche de la capacité de charge de l'environnement du système. S'il est impossible de prédire ce qui va se passer lorsque la capacité de charge de notre planète sera presque atteinte, des signes montrent que nous atteignons ces limites au moins pour les gens qui ont adopté un mode de vie moderne de forte consommation (Wackernagel et al., 2002). En effet, certains indicateurs, comme l'Index Planète vivante du WWF, montrent que nous avons déjà dépassé la capacité de charge à long terme de la planète (WWF, 2008).

Couplant les connaissances scientifiques et traditionnelles, les gens ont besoin d'outils d'évaluation subjectifs, fondés sur l'éthique, comme le cadre fourni par la Charte de la terre, pour les aider à appliquer des principes éthiques aux défis environnementaux du moment. De cette façon, l'éthique de la conservation de la biodiversité peut être intégrée dans une politique et elle peut être plus explicite dans les efforts de conservation de la biodiversité tant locale que mondiale.

Un de ces outils éthiques est le Principe de précaution, qui fut élaboré à l'origine au Sommet de la terre de 1992, à Rio. Principe 15 de la Déclaration de Rio, il déclare que « en cas de risques de dommages graves ou irréversibles, l'absence de certitude scientifique absolue ne doit pas servir de prétexte pour remettre à plus tard l'adoption de mesures effectives visant à prévenir la dégradation de l'environnement ». Désormais largement accepté, le Principe de précaution tient compte de préoccupations éthiques lors de la prise de décisions qui peuvent affecter l'environnement.

En intégrant des principes éthiques dans des processus de changements et des prises de décisions, des particuliers peuvent aider à casser les réactions en chaîne qui entraînent le système environnemental mondial vers l'effondrement. L'éthique doit devenir un véritable outil tant pour les actions collectives qu'individuelles, et il certain que l'étiquette " verte » de nombreuses sociétés indique quelques progrès dans cette direction (Chapitre 15).

Parallèlement, l'existence de cadres éthiques différents peut entraîner des malentendus et des conflits. Par exemple, les questions liées à la chasse, à l'abattage de populations sauvages, à l'utilisation d'organismes génétiquement modifiés (OGM) et au recours aux animaux pour la recherche médicale humaine, ont toutes suscité des controverses et attirent beaucoup l'attention des média. Comme les dimensions éthiques de la conservation vont s'élargir dans les années qui viennent, la communauté de la conservation devra trancher ce débat entre les valeurs intrinsèques des espèces et des écosystèmes et leur valeur instrumentale pour les gens. Ceci pourra bénéficier des recherches sur la valorisation des services environnementaux qui sont en cours (Chapitre 4), mais les efforts pour s'assurer que 
toutes les valeurs sont prises en compte seront primordiaux.

L'élaboration d'une éthique de la conservation plus solide est un moyen fondamental pour appuyer la conservation de la biodiversité à long terme. Nous devrons établir des partenariats avec des chefs religieux qui insistent de plus en plus sur l'éthique environnementale, qui est inhérente à toutes les religions. Comme l'avenir appartient à la jeune génération, nous devons nous focaliser sur les jeunes d'aujourd'hui mais nous préoccuper des questions qui concernent tout le monde. 


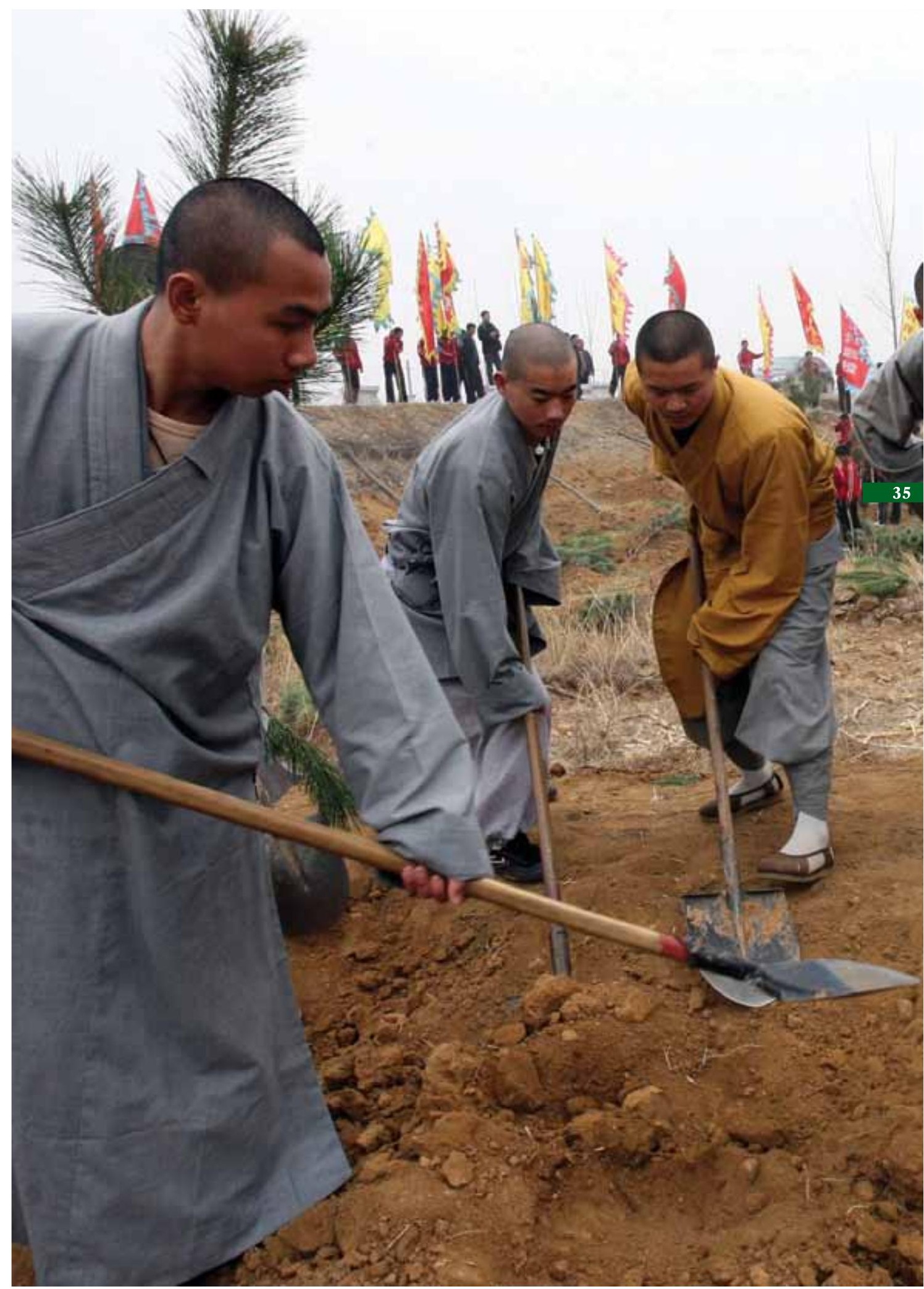


3. Le rôle central des gens

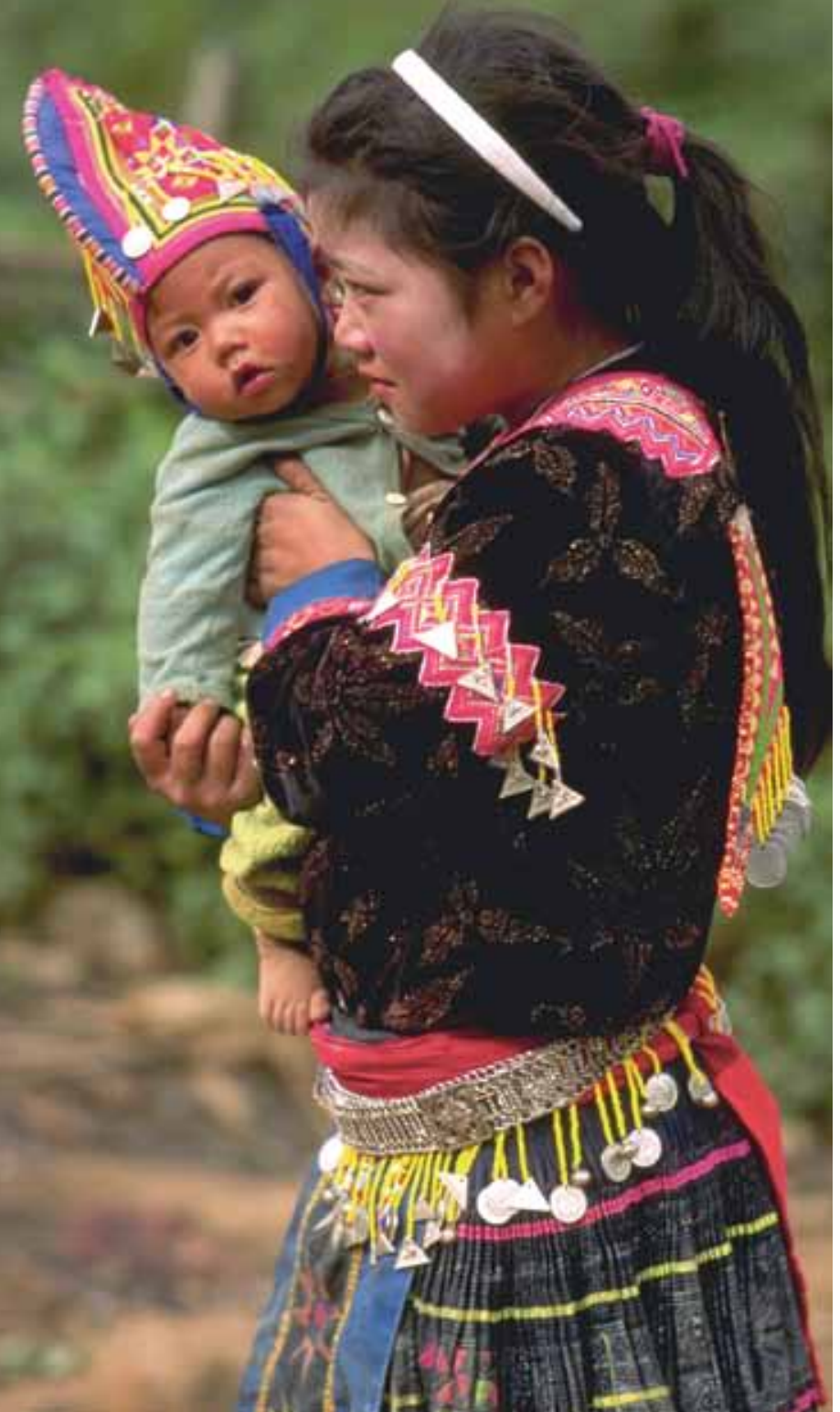


Les gens, cela peut être des

consommateurs, des entrepreneurs, des destructeurs et bien d'autres

encore. Plus des trois-quarts

de la surface de la terre libre de

glace présentent des traces de

modifications dues à la présence

humaine et à l'aménagement du

territoire. Dès les premières années

du mouvement mondial en faveur

de la conservation, la conversion

des habitats fut considérée comme

la menace principale pour la

conservation de la nature (et, dans

une certaine mesure, elle l'est

encore). Des populations furent

exclues des aires protégées (AP),

suivant ainsi le « Modèle Yellowstone »

qui a déplacé des Amérindiens de

leurs terres ancestrales au nom des

intérêts nationaux de la conservation

(imposés au début par les militaires).

Le développement humain a aujourd'hui atteint un tel point que l'aménagement du territoire est véritablement écartelé entre les aires protégées, les terres agricoles, les forêts et un urbanisme en extension. Les hommes dont les moyens de subsistance dépendent des écosystèmes montrent que les terres naturelles peuvent inclure des gens et qu'en réalité, les hommes font depuis longtemps partie de la nature. Nombreux sont ceux qui arguent que les populations locales ont des droits coutumiers sur ces ressources. La plupart des organisations de conservation reconnaissent désormais combien il est important d'intégrer ces personnes dans les efforts de conservation, même si certaines prétendent que les aires de nature sauvage, où l'empreinte humaine est éphémère, sont essentielles pour protéger au moins quelques espèces (par exemple, les grands prédateurs) et qu'il est nécessaire de préserver des habitats « intacts » pour les comparer à ceux qui ont été modifiés par l'humanité moderne.

Au cours des dernières décennies, de nombreuses organisations de conservation, dont l'UICN, ont lancé des initiatives pour atteler et concentrer la capacité institutionnelle des communautés locales à la conservation moderne de la biodiversité. Ces initiatives étaient motivées par le principe selon lequel des écosystèmes sains offrent des services essentiels pour tous les peuples. Malgré ces efforts, la désertification croissante, la perte de fertilité des sols et la pollution des eaux ont continué à réduire la capacité des écosystèmes à combler les besoins des hommes (Evaluation des écosystèmes pour le millénaire, 2005). Le rapport 2005 de l'Evaluation des écosystèmes pour le millénaire $(\mathrm{EM})$ a révélé que $60 \%$ de tous les services écosystémiques étaient dégradés. Les gens sont de moins en moins à même de s'en remettre aux écosystèmes, et ils dépendent d'un nombre toujours plus réduit de sources de nourriture. Quatre espèces végétales seulement - froment, maïs, riz et pommes de 
terre - fournissent plus de la moitié des calories d'origine végétale du régime alimentaire humain (Pirages et De Geest, 2003). Peut-être par mégarde, les hommes augmentent leur exposition aux risques de changements des écosystèmes, et la façon dont nous gérons ces risques aura un impact profond sur les résultats.

La communauté de la conservation accepte en général le postulat selon lequel la pauvreté est liée à l'état dégradé des ressources naturelles et des services écosystémiques, et les questions de la conservation et de la réduction de la pauvreté seront discutées plus en détail dans d'autres chapitres.

Les conservationnistes reconnaissent aussi que l'impact de la richesse sur ces ressources et services est évident. Les schémas de consommation, les choix en matière de développement, la distribution des richesses, les politiques gouvernementales et les technologies peuvent atténuer ou aggraver les effets environnementaux des changements démographiques. Les économies industrielles actuelles consomment des quantités insupportables d'énergie et de matières premières et produisent d'énormes volumes de déchets et de polluants. Comme le souligne le Programme des Nations Unies pour l'environnement (PNUE) (2003), la pollution et la perturbation des écosystèmes qui en résultent s'observent souvent dans des pays qui sont très éloignés du site de consommation. L'attitude et les préférences des consommateurs ont un effet profond sur l'environnement, en raison des différences des impacts environnementaux de la production, de l'utilisation et de l'élimination de certains biens et services. De plus, les préférences des consommateurs ne sont pas permanentes. Les schémas de consommation sont enracinés dans des systèmes de valeurs qu'ils contribuent à faire changer. Les cultures qui étaient jadis distinctes et relativement isolées les unes des autres sont de plus en plus interconnectées par des relations commerciales, ce qui crée une culture nouvelle, homogénéisée, fondée sur une consommation et une possession ostensibles de biens matériels. Les cultures traditionnelles qui pratiquaient l'utilisation à faible intensité de ressources naturelles sont très rapidement déplacées, ou alors elles se transforment radicalement pour se doter de ce qu'elles voient comme des avantages comparatifs indispensables pour survivre dans un monde poussé par la compétition économique. La globalisation a étendu la portée des médias et de la publicité, renforçant des systèmes de valeurs basés sur des idéaux de consommation que l'on présente comme des synonymes de bonheur et de bien-être. De tels systèmes de valeurs sont d'une durabilité douteuse et pourraient même contribuer à de l'agitation sociale.

L'intégration croissante des marchés internationaux - la mondialisation - a permis, voire encouragé la diffusion de schémas de consommation modernes des pays "développés ", avec des implications considérables pour l'environnement et la société. La façon dont ces marchés pourraient contribuer à la conservation est discutée au Chapitre 12.

Si les gens, leurs besoins et leurs souhaits peuvent être la raison pour laquelle nous sommes confrontés à ces défis urgents, ils sont aussi notre seul moyen de résoudre les problèmes. Le développement et l'adoption de l'Approche écosystémique de la Convention sur la diversité biologique (CDB) furent une confirmation explicite, par le monde de la conservation, du fait que l'attention portée aux hommes et à leurs besoins est fondamentale pour réussir (http://www.cbd.int/ecosystem/principles. shtml). L'UICN a bien accueilli ces 12 principes mais elle reconnaît que de nombreuses approches écosystémiques peuvent être compatibles avec eux, par exemple la restauration de paysages forestiers, la gestion intégrée des ressources en eau et la gestion intégrée des zones côtières.

D'autres questions doivent être incluses, comme la conservation des cultures et des savoirs traditionnels, la promotion des approches de la conservation fondées sur les droits, et 
l'engagement des communautés locales et des populations autochtones.

\section{LIER LA DIVERSITÉ CULTURELLE ET BIOLOGIQUE}

La biodiversité et la diversité culturelle se recouvrent largement, ce que certains appellent la "biodiversité culturelle » (Posey, 1999 ; Jianchu, 2000). Cette superposition est évidente sur les cartes où les « hauts-lieux » de la diversité culturelle, des zones de grande diversité culturelle, recouvrent très nettement les zones riches en biodiversité (Maffi, 2005). Ce chevauchement illustre bien le fait que le concept de nature n'est pas séparé des peuples ou de la culture, et qu'au contraire il s'y intègre.

Les hommes se sont toujours fondés sur des structures et des normes sociales, un reflet de la culture, comme protection contre les risques de changements environnementaux. Ces moyens d'adaptation sociaux peuvent prendre la forme de partages locaux des ressources, la dépendance vis-à-vis de familles ou de lignées, l'adoption d'une nouvelle technologie, la migration ou une modification du comportement. En intégrant des inventions et des pratiques venues de nombreuses cultures, les gens sont mieux équipés pour s'adapter aux changements. Si de telles ressources culturelles s'affaiblissent, les hommes auront moins de capacités de s'adapter à des conditions en évolution.

L'importance des savoirs environnementaux traditionnels et le rôle des populations autochtones et des communautés locales pour le travail de conservation sont reconnus par la CDB qui demande aux Parties de :

respecter, préserver et maintenir les connaissances, les innovations et les pratiques des communautés autochtones et locales qui incarnent des modes de vie traditionnels présentant un intérêt pour la conservation et l'utilisation durable de la diversité biologique et d'en favoriser l'application sur une plus grande échelle, avec l'accord et la participation des dépositaires de ces connaissances, innovations et pratiques et d'encourager le partage équitable des avantages découlant de l'utilisation de ces connaissances, innovations et pratiques.

La CDB légitime le savoir traditionnel comme partie d'un cadre légal mondial. En créant une approche systématique de la relation entre population et protection de l'environnement, elle montre que les hommes, la biodiversité et les paysages forment une unité complexe et intégrée. Cette approche permet des stratégies de conservation novatrices, comme l'exploration de la relation entre femmes autochtones, gestion des ressources et biodiversité.

La capacité des hommes de changer leur comportement permet aux populations de se montrer résilientes face aux changements environnementaux, de réduire leur impact sur les systèmes naturels et d'en encourager la conservation si c'est leur choix. L'UICN est en train d'intégrer activement culture et préoccupations en matière de subsistance dans tout son programme de travail. Citons comme exemples le Programme sur la restauration des paysages forestiers et la Commission mondiale des aires protégées (CMAP), par ses groupes de travail sur les Paysages protégés et sur les Valeurs culturelles et spirituelles des aires protégées. Cette perspective intégrée fait de plus en plus partie de pratiquement tous les programmes de conservation.

Un défi, avec ce glissement dans les politiques et dans la façon de penser la conservation en général, est que de nombreuses communautés locales sont supposées gérer des projets de conservation, sans disposer, le plus souvent, de l'ensemble des compétences et des capacités nécessaires pour pouvoir adopter ce qui peut être une approche peu familière de la gestion des ressources. Une solution pourrait être d'utiliser des techniques conventionnelles pour étayer et renforcer les 
capacités, les technologies et les connaissances locales qui sont déjà mises en pratique dans les communautés locales et autochtones. De nombreuses cultures locales gèrent déjà bien leurs terres et leurs ressources, mais elles ont besoin de s'adapter aux nouvelles pressions d'une société moderne et mondialisée. Le défi consistera à trouver les moyens adéquats pour intégrer les processus locaux et les pratiques de conservation conventionnelles dans les nouvelles structures de gouvernance des ressources nationales et mondiales.

\section{APPROCHES DE LA CONSERVATION FONDÉES SUR LES DROITS}

De nombreux grands projets de développement, comme la construction de barrages, l'urbanisation, des routes, des concessions d'exploitation forestière, ont accordé trop peu d'attention aux droits des populations locales concernées. De la même façon, la communauté de la conservation reconnaît que « les pratiques de conservation peuvent affecter le bien-être humain et que, parfois, elles ont porté atteinte aux droits humains, y compris aux moyens de subsistance locaux, par des violations des droits de l'homme, par des déplacements forcés et des impacts sur les modes de vie locaux, spécialement ceux des peuples autochtones et des communautés locales »(UICN, 2008e). En gardant cela à l'esprit, la vision de l'UICN « d'un monde juste qui valorise et préserve la nature » exigera l'application à tout le travail de l'Union d'approches basées sur les droits. Cela garantira une attention complète aux droits humains, aux droits d'accès à la propriété et aux ressources, et aux droits coutumiers des populations autochtones et des communautés locales.

La question des approches de la conservation fondées sur les droits est particulièrement pertinente pour les populations autochtones minoritaires qui, jusqu'il y a 30 ans à peine, avaient peu de droits dans la plupart des pays. Cela a fortement influencé la façon de penser la conservation avant cette époque, et il en reste quelque chose dans certaines pratiques de conservation actuelles. Jusqu'il y a peu, les groupes autochtones n'avaient aucun droit permanent ou formel sur leurs terres, et les organisations de conservation pouvaient donc difficilement travailler directement avec eux. Avec la progression des droits de l'homme et l'utilisation plus générale d'approches basées sur ces droits pour le développement, les droits des populations autochtones sont maintenant reconnus et mis en avant grâce à la mention explicite des valeurs du savoir traditionnel et des communautés autochtones. L'adoption, en 2003, de l'Accord de Durban par le $V^{e}$ Congrès mondial des parcs de l'UICN et la Déclaration de 2007 des Nations Unies sur les droits des peuples autochtones rendent inimaginable pour l'UICN d'encore mener des activités touchant des populations locales sans le consentement libre, préalable et informé des populations directement impliquées.

Cette attention pour les droits et le statut légal est liée au processus mondial de démocratisation. En insistant sur la transparence et la participation publique, la gestion des ressources a évolué pour devenir plus communautaire, ce qui signifie aussi bien des défis que des opportunités pour l'UICN.

\section{POPULATIONS ETÉCOSYSTÈMES, OU POPULATIONS DANS LES ÉCOSYSTÈMES}

Les communautés locales, et spécialement les populations autochtones, sont souvent les populations les plus marginalisées du point de vie politique et économique. Elles sont pourtant souvent les gardiennes des zones les plus riches du point de vue biologique. Selon Sobrevila (2008), les territoires autochtones traditionnels couvriraient quelque $22 \%$ de la surface terrestre et accueilleraient $80 \%$ de la diversité des espèces terrestres de la planète.

Les pratiques conventionnelles modernes de la conservation sont souvent rejetées par les communautés locales, spécialement lorsque ces 
dernières n'ont pas été totalement impliquées dans les prises de décisions. Il en résulte une poursuite de la dégradation des habitats et de la perte de biodiversité. Le modèle Yellowstone de parcs nationaux, par exemple, a été fermement refusé en Asie de l'Ouest, en Afrique du Nord et ailleurs. Cela ne veut pas dire que ces régions n'ont pas d'aires protégées, mais que les populations locales ont trouvé leurs propres moyens de protéger les ressources en dehors des cadres juridiques officiels ; mais certains de ces arrangements sont affaiblis parce qu'ils ne sont pas reconnus par les lois internationales et nationales. Les approches traditionnelles de la conservation, comme le système Hema dans les pâturages d'Asie de l'Ouest, peuvent être adaptées pour constituer des approches viables de la conservation dans des conditions modernes.

La plupart des programmes de conservation exigent une maintenance et une gestion de longue haleine, qui peuvent aussi tirer avantage du fait de travailler avec les communautés locales. On peut considérer la conservation comme un bien public et, à ce titre, elle devrait recevoir un financement public, mais cela est rarement suffisant (spécialement dans les pays en développement qui sont soumis aux restrictions du FMI). Les fondations, les organismes de développement, les organisations non gouvernementales (ONG) et les philanthropes ont toujours été très utiles, mais la conservation exige un soutien continu, qui dure bien plus longtemps que ce que les donateurs sont prêts à faire. Par conséquent, les projets de conservation souffrent d'affaiblissements systémiques lorsque les financements se tarissent. Pour vaincre cette difficulté, les conservationnistes sont en train de créer des liens avec les structures sociales locales et de se tourner vers les communautés locales pour obtenir leur soutien. Pour de nombreux projets, l'appropriation locale de la gestion du projet et de sa survie est d'une part plus efficace et d'autre part produit des résultats plus significatifs, comme les programmes pour les aires protégées décentralisés et supportés localement.

La nouvelle approche de la Commission mondiale des aires protégées de l'UICN vers l'autogouvernance et la gestion par les communautés autochtones est un développement prometteur qui a reconnu l'importance des communautés locales dans la gestion des aires protégées. Quelque 86\% des aires classées comme parcs nationaux en Amérique latine abritent de façon temporaire ou permanente de communautés locales ou autochtones (Amend et Amend, 1995), de sorte que l'on peut dire que cette approche capitalise sur la présence déjà forte de communautés autochtones à l'intérieur et à l'extérieur des aires protégées. L'auto-gouvernance des ressources locales peut aussi aider à réduire la pauvreté des communautés locales, notamment par des opportunités comme l'intégration de la conservation et du tourisme.

Le travail avec les communautés locales doit impliquer un soutien pour s'engager dans des défis liés à l'environnement, comme les changements climatiques, les espèces envahissantes, des moyens de subsistance durables et la santé. Chacun d'eux sera discuté en détail dans d'autres chapitres. Le savoir traditionnel est une base importante pour l'adaptation aux changements climatiques et pour la réduction de la vulnérabilité face aux événements extrêmes, et sa disparition peut augmenter la fragilité des populations locales face aux changements (Ford, 2006).

Impliquer toutes les parties prenantes dans la conservation va exiger le développement d'outils et de compétences, en fournissant notamment aux gestionnaires des ressources des manuels, de l'assistance technique et d'autres moyens d'orientation pratiques facilement accessibles sur la façon d'équilibrer la gestion des ressources naturelles avec les besoins de développement économique. Il faut faciliter l'accès aux compétences complémentaires nécessaires pour arriver à réduire durablement la pauvreté 
et à atteindre un développement durable. Les

communautés locales doivent pouvoir préserver et gérer les ressources naturelles dont elles dépendent et améliorer leur coopération avec les institutions voisines lorsqu'il s'agit de gérer des ressources largement répandues.

Dans le même temps, il faut encourager les gouvernements à améliorer les droits fonciers, à donner un statut légal aux titres collectifs des populations autochtones et à permettre à la société civile de gérer les ressources naturelles renouvelables pour une utilisation durable, grâce à des droits d'accès basés sur une équité sociale et sexuelle. Les donateurs et les gouvernements devraient mettre au point et appliquer, dans les activités qu'ils financent en vue de réduire la pauvreté, des politiques qui intègrent la conservation de l'environnement et de la biodiversité. Enfin, il est urgent de définir, en matière de conservation et de droits de l'homme, une politique qui inclue des approches de la conservation fondées sur ces droits, des méthodes de mise en œuvre, des moyens d'encourager le partage des expériences, et qui définisse les responsabilités des gouvernements, des communautés, du secteur privé et des organisations de conservation. 


\section{Services écosystémiques : les avantages que les hommes reçoivent de la nature}

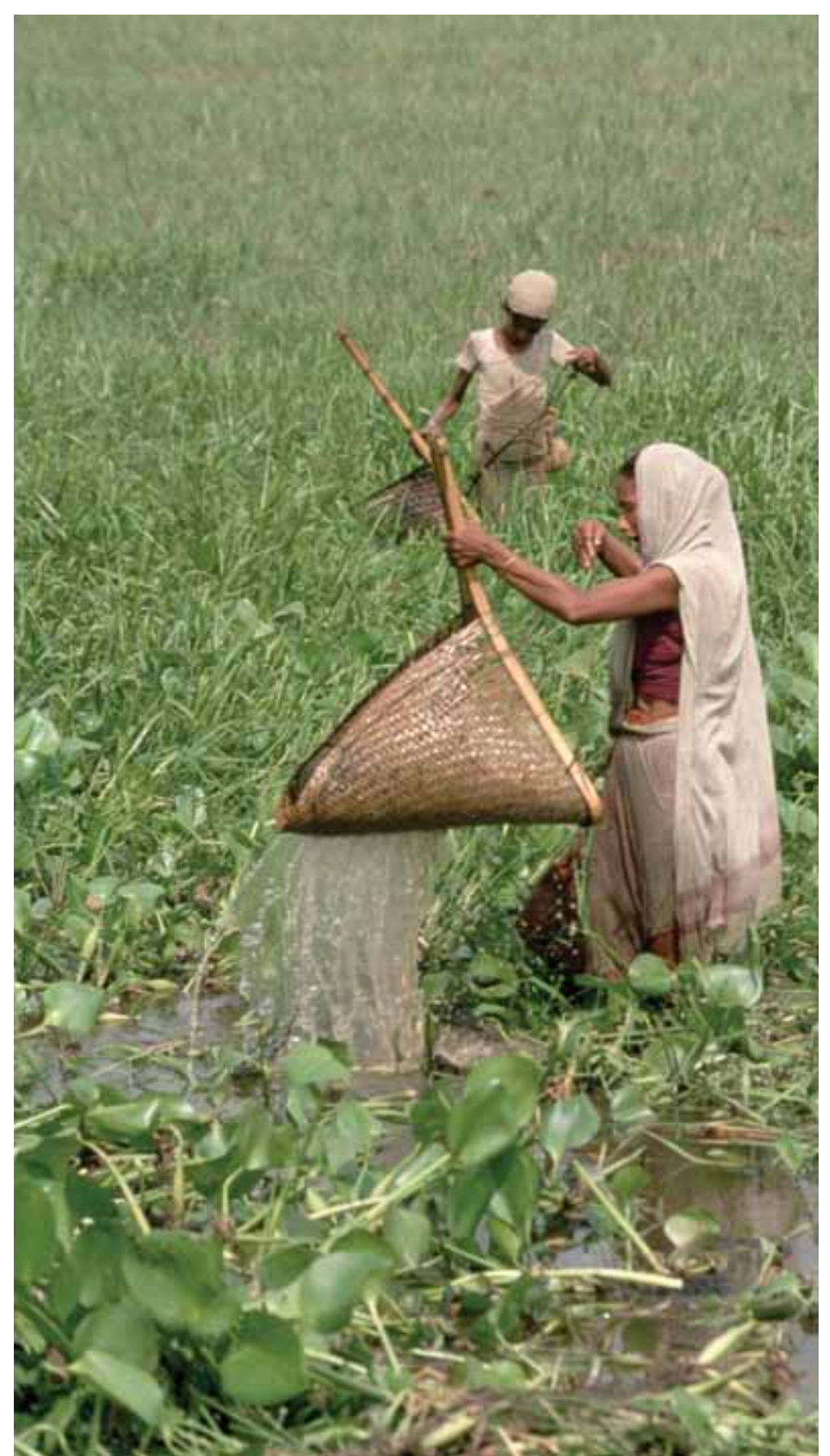


Les écosystèmes soutiennent les processus qui purifient l'air et l'eau, fécondent les cultures, décomposent les déchets, contrôlent les nuisibles toxiques et les maladies et régulent les événements naturels extrêmes. L'eau, la nourriture, les fibres, les carburants et les plantes médicinales sont tous produits par la trame complexe de la vie. L'inspiration des arts, des cultures et des religions vient la nature, qui est aussi source de loisirs et d'enrichissement spirituel.

La vie sur terre persiste depuis plus de deux millions d'années ; elle a formé les écosystèmes qui sont à l'origine des flux de nutriments, les interactions proies-prédateurs qui ont fait progresser l'évolution, et même l'atmosphère qui permet la vie sur la terre. Alors que les humains évoluaient, nos ancêtres ont bénéficié de nombre de ces fonctions élémentaires qui ont permis à notre espèce d'atteindre son niveau actuel de diversité culturelle. Avec l'émergence de la civilisation, grâce à l'apparition de l'agriculture irriguée, l'humanité a commencé à se rendre compte des avantages d'un spectre beaucoup plus large de services écosystémiques et des risques qu'il $\mathrm{y}$ avait à les malmener. Platon, par exemple, en $400 \mathrm{AC}$, reconnaissait déjà que la déforestation provoquait de l'érosion et l'assèchement des sources (Goldin, 1997). Les traités de médecine arabes du $9^{\text {ème }}$ siècle rapportent des réflexions élaborées concernant des techniques agricoles, comme l'irrigation et la rotation des cultures, mais aussi le contrôle de la pollution (Watson, 1983). Les civilisations d'Inde, de Chine et d'Asie du Sud-est mobilisaient l'eau et des algues fixant l'azote, pour créer des écosystèmes irrigués destinés à la culture du riz, qui ont produit les plus riches cultures du monde de ces temps lointains (McNeely et Wachtel, 1988).

Plus récemment, le mariage de la science et de la technologie a mobilisé l'énergie des combustibles fossiles et l'a appliquée à l'agriculture et à la production, générant suffisamment de nourriture et d'autres produits pour supporter le quadruplement de la population humaine mondiale au $20^{\text {eme }}$ siècle. Pourtant, alors que la croissance de la population s'accélérait, on s'est de plus en plus inquiété du fait qu'il y avait peut-être des limites à la croissance (Malthus, 1798 ; Meadows et al., 1972). Plus récemment, l'Évaluation des écosystèmes pour le millénaire (EM) a réuni plus de 1300 scientifiques pour rendre compte du statut d'une vaste gamme de services écosystémiques dans le monde et des conséquences des changements des écosystèmes pour les hommes, maintenant et dans le futur (EM, 2005b). Sa conclusion révèle que $60 \%$ des services écosystémiques évalués étaient dégradés ou utilisés de manière non durable à l'échelle globale et elle constitue une base scientifique raisonnable pour justifier l'urgence de conserver la biodiversité et les écosystèmes. 


\section{CLASSIFICATION DES SERVICES ÉCOSYSTÉMIQUES}

Que sont donc les services écosystémiques ?

Ce sont simplement les avantages que les écosystèmes procurent aux populations.

Le concept $d$ '« écosystème » met en évidence les interactions entre les composantes de la biodiversité à différents niveaux et les interactions entre les espèces vivantes et l'environnement abiotique. En effet, ces interactions soutiennent, régulent et fournissent des avantages que les hommes retirent de la biodiversité. Les gens ne retirent pas les services de différents niveaux de la biodiversité de façon indépendante ; au contraire, les services sont fournis par les écosystèmes et par des éléments qui en font partie, le tout fonctionnant comme un ensemble. Lorsque le système est dégradé, les services sont moins nombreux. Cela justifie amplement l'attention de l'UICN pour la préservation des écosystèmes, des services qu'ils fournissent et de la biodiversité qui les soutient.

Les hommes dégradent souvent les écosystèmes, mais nous pouvons les restaurer et nous pouvons intervenir de façon raisonnable dans leur gestion pour changer l'équilibre et assurer la multiplicité

FIGURE 4.1 CADRE DE CLASSIFICATION DES SERVICES ÉCOSYSTÉMIQUES (EM, 2003)

\section{APPROVISIONNEMENT}

Biens produits ou fournis par les écosystèmes

- Nourriture

- Eau douce

- Bois de feu

- Fibres

- Produits biochimiques

- Ressources génétiques

\section{RÉGULATION}

Avantages tirés de la régulation des processus de l'écosystème

- Régulation du climat

- Régulation des maladies

- Régulation des flux d'eau

- Détoxication

\section{CULTURE}

Avantages non matériels obtenus des écosystèmes

- Spirituels

- Récréatifs

- Esthétiques

- Créatifs

- Éducatifs

- Communautaires

- Symboliques

\section{SOUTIEN}

Services nécessaires à la production d'autres services écosystémiques

- Formation des sols

- Cycle des nutriments

- Production primaire

des services. Qu'ils soient gestionnaires de zones forestières, de zones humides, d'exploitations agricoles, ou encore jardiniers amateurs, les gens réalisent qu'ils gèrent des écosystèmes. Même ceux qui sont focalisés sur la conservation d'espèces dans la nature reconnaissent qu'aucune espèce n'est une île, totalement indépendante ; sa survie dépend au contraire de ses relations avec les autres composantes de l'écosystème dont elle fait partie.

L'Evaluation des écosystèmes pour le millénaire (EM, 2003) a classé les services écosystémiques en quatre groupes : les services de soutien, de régulation, d'approvisionnement et de cadre culturel (Figure 4.1). 


\section{SERVICES DE SOUTIEN}

Les services de soutien incluent la production primaire, le cycle des nutriments, le cycle de l'eau, la pollinisation et la fourniture d'habitats. Leurs avantages pour les gens sont indirects et ils permettent aux écosystèmes de fournir des services culturels, de régulation et d'approvisionnement. Par exemple, le service d'approvisionnement que constitue la production de nourriture dépend des services de soutien que sont le cycle des nutriments, la formation des sols, le cycle de l'eau et la pollinisation. D'un point de vue économique, cela n'a aucun sens de valoriser directement les services de soutien dans la mesure où la valeur de ceux-ci devrait être (mais souvent n'est pas) intégrée à la valeur des bénéfices directs que nous tirons des écosystèmes (par exemple, la nourriture ou l'eau).

\section{SERVICES D'APPROVISIONNEMENT}

Les services d'approvisionnement sont les biens produits par les écosystèmes. Ce sont ceux qui sont les plus directement reconnaissables pour la plupart des gens, et ce sont les bénéfices les plus tangibles tirés des écosystèmes (encore que, comme nous l'avons mentionné plus haut, ils dépendent totalement des services de soutien). Allant des ressources génétiques que sont les espèces sauvages jusqu'aux plantes cultivées et aux animaux dont nous dépendons pour une grande part de notre alimentation, les services d'approvisionnement fournissent aussi des moyens de subsistance qui vont au-delà des produits de la consommation directe, parce qu'ils sont facilement valorisés et échangés sur les marchés. Bien que le processus soit rarement reconnu comme tel, les gens participent au paiement des services écosystémiques chaque fois qu'ils achètent de la nourriture, du bois de feu ou des remèdes naturels. Les services d'approvisionnement répondent aux besoins de la population mondiale en nourriture, en fibres naturelles, en médicaments et en ressources génétiques, et ils répondent aux besoins en combustibles d'un tiers de la population mondiale qui n'a pas accès aux combustibles fossiles.

\section{SERVICES DE RÉGULATION}

Les services de régulation sont les avantages qui résultent des différentes façons dont les écosystèmes influencent l'environnement où nous vivons. Ils comprennent la régulation de la qualité de l'air et des climats, de la qualité et de la quantité de l'eau, des nuisibles et des maladies, des tempêtes et autres aléas naturels. Les services de régulation opèrent sur une large gamme d'échelles ; par exemple, la régulation des systèmes climatiques se fait à l'échelle mondiale, celle des flux d'eau à l'échelle d'un bassin de rivière, et celle des vents et des tempêtes se fait à une échelle tout à fait locale. Il n'est pas évident d'évaluer les services de régulation en termes économiques, et ils sont rarement pris en compte dans les systèmes de comptabilité nationaux. On peut aussi considérer certains services de régulation comme des services de soutien, selon la façon dont des changements de ces services affectent les hommes. Par exemple, alors que la fixation et la formation des sols régulent directement la qualité de l'eau, les hommes peuvent aussi profiter indirectement de la formation des sols, par exemple, une fois encore, par le service d'approvisionnement qu'est la production de nourriture. Des économistes travaillent aujourd'hui sur des approches qui permettront d'attribuer une valeur à ces services et qui mèneront à de nouvelles formes de paiement pour les services écosystémiques (PES).

\section{SERVICES CULTURELS}

Les services culturels sont les avantages non matériels, et parfois intangibles, que les hommes retirent des écosystèmes. Ils incluent les avantages que les hommes trouvent dans l'esthétique et l'inspiration, dans les aspects spirituels et religieux des écosystèmes, l'éducation et la science, et aussi l'affinité culturelle et les valeurs de patrimoine que de nombreuses personnes associent à des paysages et à certaines 
espèces, spécialement dans les régions où elles vivent. Les services culturels sont étroitement liés aux valeurs et aux comportements humains et ils peuvent varier significativement selon les perspectives, sociales, économiques et politiques. Bien que les valeurs culturelles et autres avantages intangibles des écosystèmes soient souvent difficiles à évaluer, ils apportent néanmoins des bénéfices fondamentaux pour les individus et les sociétés de par le monde. Quelles que soient la valeur que nous pourrions attribuer à l'existence d'espèces particulières comme le panda ou la baleine, l'idée scientifique que nous nous forgeons en observant la nature, ou les affinités spirituelles et culturelles que de nombreuses personnes ressentent avec certaines forêts sacrées ou des espèces emblématiques, les services culturels des écosystèmes font du bien à nos corps, à nos esprits et à nos âmes. Les bénéfices récréatifs et touristiques de la nature et de la biodiversité, contrairement à de nombreux autres services culturels, sont aisément mesurables et quantifiables en termes économiques. Ils sont devenus une source majeure de revenus au niveau local, régional et national, et ils contribuent significativement à l'amélioration de la qualité de vie des communautés locales même s'ils impliquent des compromis.

\section{POLITIQUE ET PLANIFICATION POUR LES SERVICES ÉCOSYSTÉMIQUES}

La meilleure appréhension de l'importance et de la valeur des services écosystémiques de ces 20 dernières années a suscité une série d'événements clés et il en est résulté d'importantes initiatives en matière de politique. La plus notable fut la Conférence des Nations Unies sur l'environnement et le développement, connue sous le nom de Sommet de la terre, qui s'est déroulée à Rio de
Janeiro, au Brésil, en juin 1992. Un des résultats majeurs du Sommet de la terre fut la Convention sur la diversité biologique (CDB) qui compte aujourd'hui 191 Etats Parties. La notion de services écosystémiques est profondément enracinée dans la CDB grâce aux concepts " d'utilisation durable » et de « bénéfices » spécifiés dans ses objectifs.

Les services écosystémiques furent aussi reconnus lorsque l'Assemblée générale des Nations Unies a adopté les huit Objectifs du millénaire pour le développement (OMD) en 2000. Le septième objectif, "Assurer un environnement durable ", vise explicitement le maintien des services écosystémiques et la conservation de la biodiversité (Melnick et al., 2005). Deux ans plus tard, le Sommet mondial sur le développement durable (SMDD) qui s'est déroulé à Johannesburg, en Afrique du Sud, a appuyé les Objectifs du millénaire pour le développement et a consolidé le thème du progrès dans cette direction comme composante centrale de la politique intergouvernementale.

Au niveau des nations aussi, on a adopté des politiques pour préserver la fourniture de services écosystémiques. Une recherche dans la base de données ECOLEX (effort conjoint de l'UICN, la FAO et le PNUE) ne relève pas moins de 602 mentions de "services écosystémiques » dans des législations nationales. Les services d'approvisionnement sont les plus fréquents, peut-être parce que ce sont les plus évidents et les plus faciles à mesurer et qu'ils génèrent le plus de revenus d'impôts.

L'autre réponse politique majeure à la reconnaissance des valeurs des services écosystémiques fut le développement d'un marché pour ces derniers. Le Costa Rica est un 
bon exemple de pays qui a fait les premiers pas pour développer des marchés pour des services écosystémiques (Rojas et Aylward, 2003). Ce pays fut longtemps un leader mondial dans l'industrie de l'écotourisme - en réalité, il vend des services écosystémiques touristiques. Au cours des années 1990, il lança des systèmes par lesquels les communautés et les sociétés en aval des cours d'eau payaient les occupants de l'amont pour l'entretien et la restauration des forêts d'où provenait leur eau. Plus récemment, le Costa Rica s'est montré très actif, comme d'autres pays tropicaux, dans le développement d'incitants et de financements pour la Réduction des émissions issues de la déforestation et de la dégradation (REDD), en tant qu'approche pour s'approprier les bénéfices économiques de la séquestration de carbone et du stockage de biomasse pour la régulation du climat.

Etant donné l'importance que prennent les services écosystémiques dans les politiques et les marchés, les Etats, les gouvernements locaux et les organisations non gouvernementales (ONG) cherchent des approches pour fournir au mieux des services écosystémiques. La clé d'une planification efficace est une présentation claire des objectifs. Par exemple, l'objectif d'un organisme donné peut être de réduire de moitié la proportion des gens qui n'ont pas accès à l'eau potable (c'est une partie de l'Objectif 7 du millénaire pour le développement). On peut alors récolter des données et élaborer des modèles pour documenter les options qui pourraient permettre d'atteindre cet objectif, comme l'installation d'infrastructures (pour la purification ou la désalinisation), l'amélioration des installations sanitaires, et la maintenance de l'habitat forestier au sein des bassins de rivières. Les différentes combinaisons et configurations spatiales de ces options auront un coût et des avantages différents. Les coûts comprendront non seulement la construction et l'entretien, le suivi et l'évaluation directs, mais aussi les coûts d'opportunité (ex. l'entretien d'habitats forestiers peut exiger de renoncer à des coupes de bois). Les bénéfices devront être directs, et contribuer alors à l'objectif déclaré, et indirects, lorsque les services écosystémiques peuvent être " groupés » ou « dégroupés » pour pousser d'autres partenaires à investir dans le plan (ex. le maintien d'habitats forestiers va aussi entraîner des REDD). Avec les données en main, on peut alors recourir à l'analyse spatiale coût-bénéfice et/ou à des systèmes d'enchères inversées pour produire un plan qui atteint l'objectif au moindre coût (ou arrive aussi près de l'objectif que possible pour un budget donné).

\section{ESTIMATION ET AU-DELÀ}

Le concept de services écosystémiques vise à mettre en lumière les déséquilibres actuels des forces du marché, qui attribuent un poids supérieur aux biens et services commercialisés mais qui ont tendance à négliger les valeurs des écosystèmes et les autres avantages relevant du non-marchand. En encourageant la prise de conscience quant à la valeur complète des services écosystémiques, les conservationnistes espèrent que les décideurs politiques vont agir et que les marchés pourront être transformés afin de mieux refléter la véritable relation entre bien-être humain et santé de l'écosystème, et par conséquent, de soutenir la conservation de la nature. Cela implique souvent d'estimer la valeur monétaire de services écosystémiques bien définis, afin d'établir un dossier économique en faveur du changement, et ensuite d'introduire des mécanismes tels que des paiements pour des services écosystémiques, qui peuvent transformer la valeur potentielle en cashflow réel et modifier les comportements.

La première étape consiste à évaluer les services écosystémiques. C'est exactement ce qu'est en train de faire l'étude de l'Économie des écosystèmes et de la biodiversité (TEEB). Un rapport intermédiaire de cette étude (CE et BMU, 2008) déclare que le bien-être humain dépend totalement de « services 
écosystémiques ». Cependant, comme ces services sont essentiellement des biens publics, sans droits de propriété bien établis, sans marché ou sans prix, ils ne sont ni reconnus ni correctement intégrés dans notre politique économique ni dans nos décisions. Une compréhension insuffisante des coûts et des avantages totaux de la conservation conduit donc à une poursuite de la perte de biodiversité. La TEEB, grâce au développement et à la diffusion d'outils économiques pour appuyer l'estimation des services écosystémiques, espère rectifier cette situation.

Cependant, de nombreuses personnes refusent une vue purement utilitaire de la nature et elles insistent sur les valeurs morales ou intrinsèques de la biodiversité. Si de telles valeurs sont certes difficiles à mesurer, il existe des approches bien établies pour les refléter dans une politique (ex. par une législation concernant la protection d'espèces menacées). La valeur intrinsèque de la nature peut être vue à la même lumière que d'autres valeurs morales ou culturelles - celles des œuvres d'art célèbres peut-être, ou des droits de l'homme.

Les racines de l'appréciation de la valeur intrinsèque de la biodiversité sont ancrées très profondément dans de nombreuses visions culturelles et religieuses $\mathrm{du}$ monde. Toutes les religions du monde ont intégré des notions de gestion responsable ou de précaution pour la nature (Gardner, 2002), comme le font de nombreux philosophes politiques connus, même si ces valeurs ne sont pas réparties équitablement entre les diverses composantes $\mathrm{du}$ monde naturel (ex. dans la plupart des cultures, les espèces animales charismatiques se voient attribuer beaucoup plus de valeur intrinsèque que des plantes ou des microorganismes). Parmi les penseurs contemporains, E.O. Wilson (1984) est celui qui a parlé avec le plus de force de valeur intrinsèque, en expliquant en détail comment il en explorait la base en termes d'évolution.

La place accordée à la valeur intrinsèque de la nature dans ce monde de développement économique et de valorisation des services écosystémiques peut être identifiée grâce à la clarté des objectifs. L’objectif du développement est d'améliorer le bien-être humain. L'objectif de la conservation de la biodiversité est de préserver et de restaurer la biodiversité et les écosystèmes, principalement en garantissant que les taux d'extinction irréversible d'espèces ne dépassent pas le niveau naturel. L'évaluation des services écosystémiques peut contribuer au bien-être humain et à la conservation de la biodiversité, un moyen pour deux fins certes, mais pas une fin en soi.

Le concept de services écosystémiques s'est attiré quelques critiques (McCauley, 2006) mais celles-ci peuvent toutes être réfutées (McNeely et al., 2009) :

- Certains biotopes peuvent produire peu d'autres services écosystémiques que des bénéfices culturels, et la stratégie est alors peu efficace, par exemple pour aider à conserver les quelque 2000 plantes endémiques du Succulent Karoo en Afrique du Sud. Mais cette région peut être spécialement importante pour ses valeurs d'option, et ses valeurs culturelles sont considérables pour les Sud-africains. Les arguments fondés sur des services écosystémiques ne s'expriment pas seulement en termes financiers.

- Les marchés fluctuent beaucoup (comme 2008 l'a prouvé dans le monde entier). Devrions-nous pour autant liquider nos actifs naturels si un marché inconstant leur attribuait demain une valeur inférieure à celle qu'ils ont aujourd'hui ? Certainement pas, parce que les services écosystémiques ne se prêtent pas tous à une évaluation de nature économique et que le concept pousse à considérer toutes les valeurs intrinsèques, culturelles et économiques, lors des prises de décisions. La combinaison des valeurs intrinsèques et économiques est plus significative que ces valeurs prises séparément.

- Le fait de dépendre de services écosystémiques comme fondements de la conservation pourrait ouvrir la porte à des arguments selon lesquels 
nous pourrions nous passer des écosystèmes si/ quand on peut fabriquer des méthodes moins coûteuses pour fournir les mêmes services. Cette préoccupation peut être atténuée lorsque nous reconnaissons que les écosystèmes fournissent des services multiples, qui doivent tous être pris en compte lors des prises de décisions.

- Il va certainement y avoir des situations où l'on pourra tirer plus de bénéfices économiques locaux en détruisant la nature qu'il n'y en aura au niveau mondial en la conservant (par exemple, l'introduction de la perche du Nil dans le lac Victoria a boosté les économies locales mais dévasté les espèces de poissons endémiques $\mathrm{du}$ lac). De tels compromis sont fréquents dans la gestion de ressources, mais l'analyse de la séquence complète des services écosystémiques permet de mieux documenter les décisions concernant les compromis qu'il faut prendre en compte. L'expérience du Costa Rica montre comment le concept de services écosystémiques peut donner des résultats pratiques positifs.

Oates (1998) ajoute un cinquième sujet de préoccupation : l'influence « corruptrice » de l'économie sur l'entreprise de la conservation ellemême. Mais le concept de services écosystémiques est utile dans de nombreux domaines de la gestion des ressources. Reid et al. (2006) soulignent que « notre planète est une mosaïque de systèmes qui fournissent aux gens différents ensembles de services et de préjudices écosystémiques. Nous ne pouvons pas gérer ces systèmes efficacement si nous ne cherchons pas à mesurer les flux de ces services, à examiner qui en bénéficie, et à envisager toute une gamme de politiques, d'incitants, de technologies et de régulations qui peuvent favoriser une meilleure gestion et un partage des bénéfices ».

Tout instrument peut être mal utilisé, mais de récentes analyses indiquent que le bon usage de l'évaluation et du paiement pour les services écosystémiques peut être très profitable pour la conservation. Une nouvelle preuve, en particulier, suggère une correspondance spatiale forte (Turner et al., 2007 ; Polasky et al., 2008) ou au moins modérée (Chan et al., 2006 ; Naidoo et al., 2008) entre la priorité accordée à la conservation de la biodiversité et l'évaluation des services écosystémiques. Cela montre que l'investissement dans la conservation des régions dont la biodiversité est une grande priorité peut aussi générer au moins certaines grandes valeurs en matière de services écosystémiques. Etant donné la concentration des domaines prioritaires tant pour la conservation de la biodiversité que pour la nécessaire réduction de la pauvreté sous les Tropiques, la conservation fondée sur le concept de services écosystémiques fournit à l'UICN et aux autres organisations de conservation une voie productive à suivre pour un avenir durable.

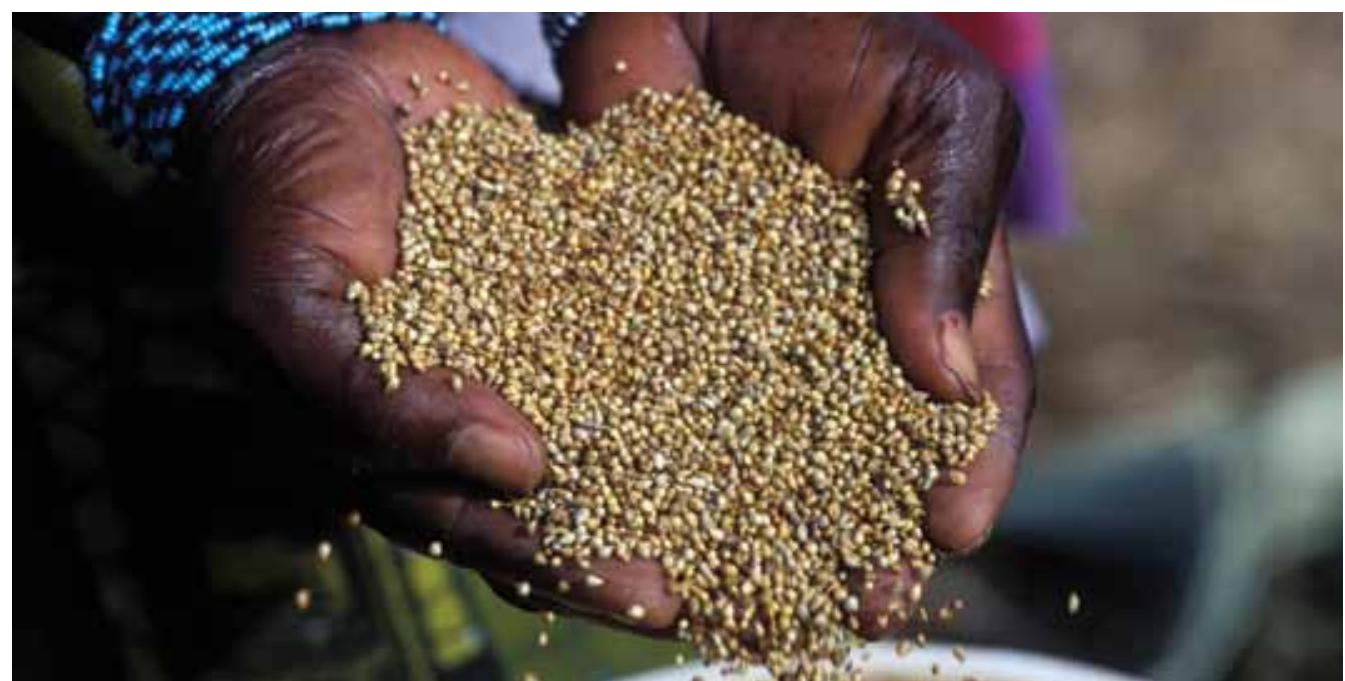




\section{Changements climatiques et biodiversité}

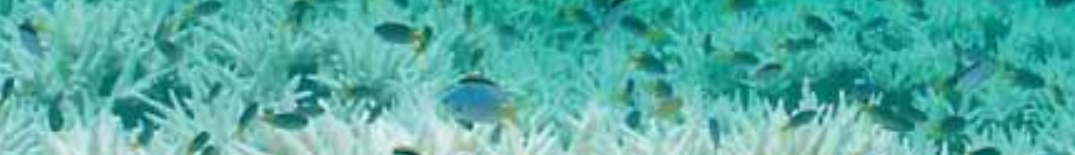

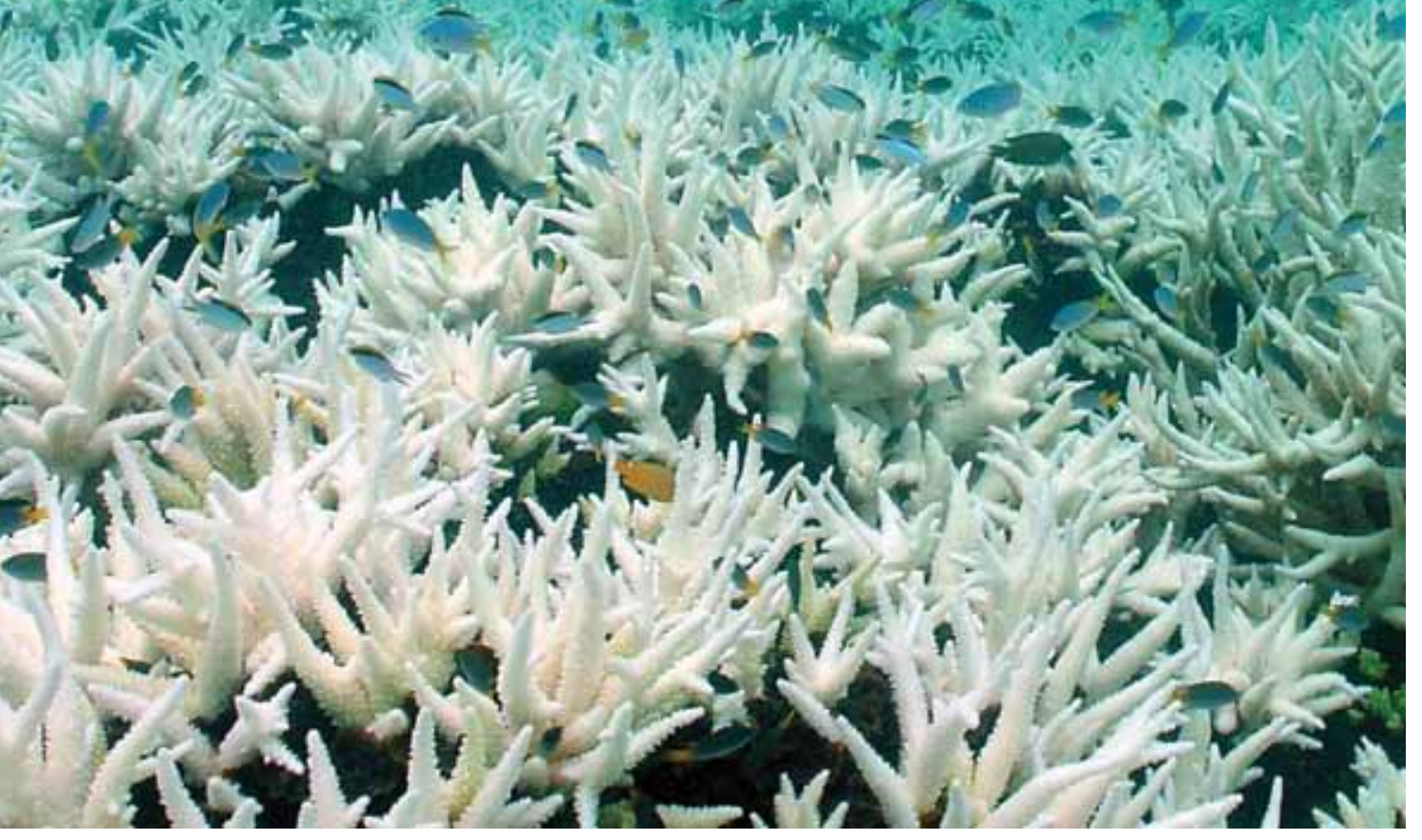


Le Groupe d'experts intergouvernemental sur l'évolution du climat (GIEC) a publié son 4⿳亠ेme Rapport d'évaluation en 2007, qui vient renforcer les résultats antérieurs selon lesquels les récentes émissions de gaz à effet de serre (GES) excèdent de loin les valeurs préindustrielles, et qui ajoute que les émissions ont même grimpé plus vite que jamais pendant la décennie 1995 - 2005 (GIEC, 2007a). Les isothermes (les lignes qui relient les endroits qui ont la même température) glissent au rythme de $40 \mathrm{~km}$ par décennie dans l'hémisphère nord et, à ce rythme, avec la conjugaison de facteurs comme la perte d'habitats et la pollution, certaines espèces auront de réelles difficultés à s'adapter (Hansen et al., 2006).

Encadré 5.1 Qu'est-ce qui change dans les changements climatiques?

Niveaux de gaz à effet de serre plus élevés

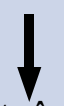

Entraînant

Températures plus élevées

Hausse du niveau des mers

Modification des courants océaniques

Modification de la chimie de l'air et des océans Evénements climatiques extrêmes

Au point de vue de la biodiversité, les résultats clés du rapport comprennent :

- Le réchauffement du climat est sans équivoque, ainsi qu'il ressort maintenant clairement des observations de l'augmentation de la température mondiale de l'air et des océans, de la fonte généralisée des calottes glaciaires et des glaciers et de la hausse du niveau moyen des mers.

- Les preuves relevées sur tous les continents et dans la plupart des océans montrent que de nombreux systèmes naturels sont affectés par des changements climatiques régionaux, et spécialement par des hausses de températures.

- La plus grande part de la hausse des températures mondiales moyennes constatée depuis le milieu $\mathrm{du} 20^{\text {ème }}$ siècle est très probablement due à l'augmentation observée des concentrations anthropogènes de GES.

Des preuves plus récentes présentées par les scientifiques du GIEC en mars 2009 indiquent que même les chiffres terribles présentés dans le $4{ }^{\text {ème }}$ Rapport d'évaluation étaient trop optimistes. Le réchauffement entre 2000 et 2007 est sans précédent, et il est dû à la croissance économique rapide de la Chine et de l'Inde, alimentée largement par du charbon. 
Solomon et al. (2009) rapportent que la gravité des impacts résultant des changements climatiques est liée non seulement à l'amplitude de ces changements, mais aussi à leur irréversibilité possible si un point de basculement climatique est atteint, au-delà duquel la nature ne pourrait plus s'adapter. Ils concluent que les changements climatiques induits par les émissions de dioxyde de carbone seront en grande partie irréversibles pendant quelque 1000 ans peut-être après la fin des émissions, à cause du temps requis pour que la température baisse après la réduction des émissions.

Quand les Délégués se sont retrouvés en avril 2009 à Bonn, en Allemagne, pour une réunion préparatoire à la COP 15 de la Convention-cadre des Nations Unies sur les changements climatiques (CCNUCC) de Copenhague en décembre 2009, la nouvelle de l'effondrement de la Plateforme de glace de Wilkins, une plaque de glace de la taille de Manhattan dans l'Antarctique, était dans tous les esprits, tel un indicateur dramatique de l'accélération des changements climatiques, et elle a imprimé un sens de l'urgence encore plus intense dans les négociations en cours.

La projection des impacts de la hausse des températures est présentée graphiquement à la Figure 5.1 .

A eux seuls, les changements climatiques causent déjà d'importantes modifications dans notre environnement. Cependant, ils sont aussi des multiplicateurs et des accélérateurs de risques amplifiant les impacts des autres menaces sur la biodiversité qui stressent déjà la nature, comme la dégradation des habitats, la pollution, les espèces envahissantes, l'émergence de maladies infectieuses et la surexploitation. En fin de compte, la fourniture de pratiquement tous les services écosystémiques sera affectée, pour certains plus que pour d'autres. La réponse humaine à ces changements sera un sujet de préoccupation majeur dans les décennies qui viennent.

Grâce au sentiment d'urgence communiqué par les médias et les rapports scientifiques, le climat est maintenant considéré comme le problème de tous - le monde des affaires, les conservationnistes, les communautés locales, les gouvernements municipaux, les propriétaires privés, les gestionnaires d'aires protégées et beaucoup d'autres. Des partenariats entre ces divers secteurs de la société, suscités par l'action en faveur du climat, pourraient aussi être bénéfiques pour la biodiversité. Il faut agir maintenant, aussi bien pour gérer les impacts évitables par des mesures d'atténuation que, de plus en plus, pour faire face aux impacts inévitables en s'y adaptant.

\section{CHANGEMENTS CLIMATIQUES ET BIODIVERSITÉ}

Pour la biodiversité, l'impact des changements climatiques est déjà évident. Une recherche portant sur 1700 espèces a montré que la nature suivait la tendance du climat, avec un glissement des aires de répartition qui est en moyenne de $6,1 \mathrm{~km}$ en direction des pôles par décennie, et que les manifestations du printemps avançaient de 2,3 jours par décennie. Ce sont des preuves évidentes du fait que le climat modifie déjà le monde de la nature (Parmesan et al., 2003).

Le dernier Atlas climatique des oiseaux nicheurs d'Europe indique que la distribution potentielle de la reproduction de la plupart des oiseaux nicheurs d'Europe va glisser de plusieurs centaines de kilomètres vers le Nord (Huntley et al., 2007). Un monde qui se réchauffe ne devrait pas très bien réussir à de nombreuses espèces à sang froid, comme les reptiles (Kearney et al., 2009). De plus, des disparitions et des extinctions d'espèces d'amphibiens sont d'ores et déjà liées aux changements climatiques (Ron et al., 2003 ; Burrowes et al., 2004 ; Pounds et al., 2006). L'on s'attend à ce que des poissons marins soient touchés par la hausse de la température de l'eau, qui modifiera la teneur en oxygène des océans (Poertner et Knust, 2007), et l'augmentation du dioxyde de carbone va augmenter l'acidité des océans, avec de graves impacts sur certaines communautés marines 
Évolution de la température moyenne par rapport à la période 1980-1999 (oC)
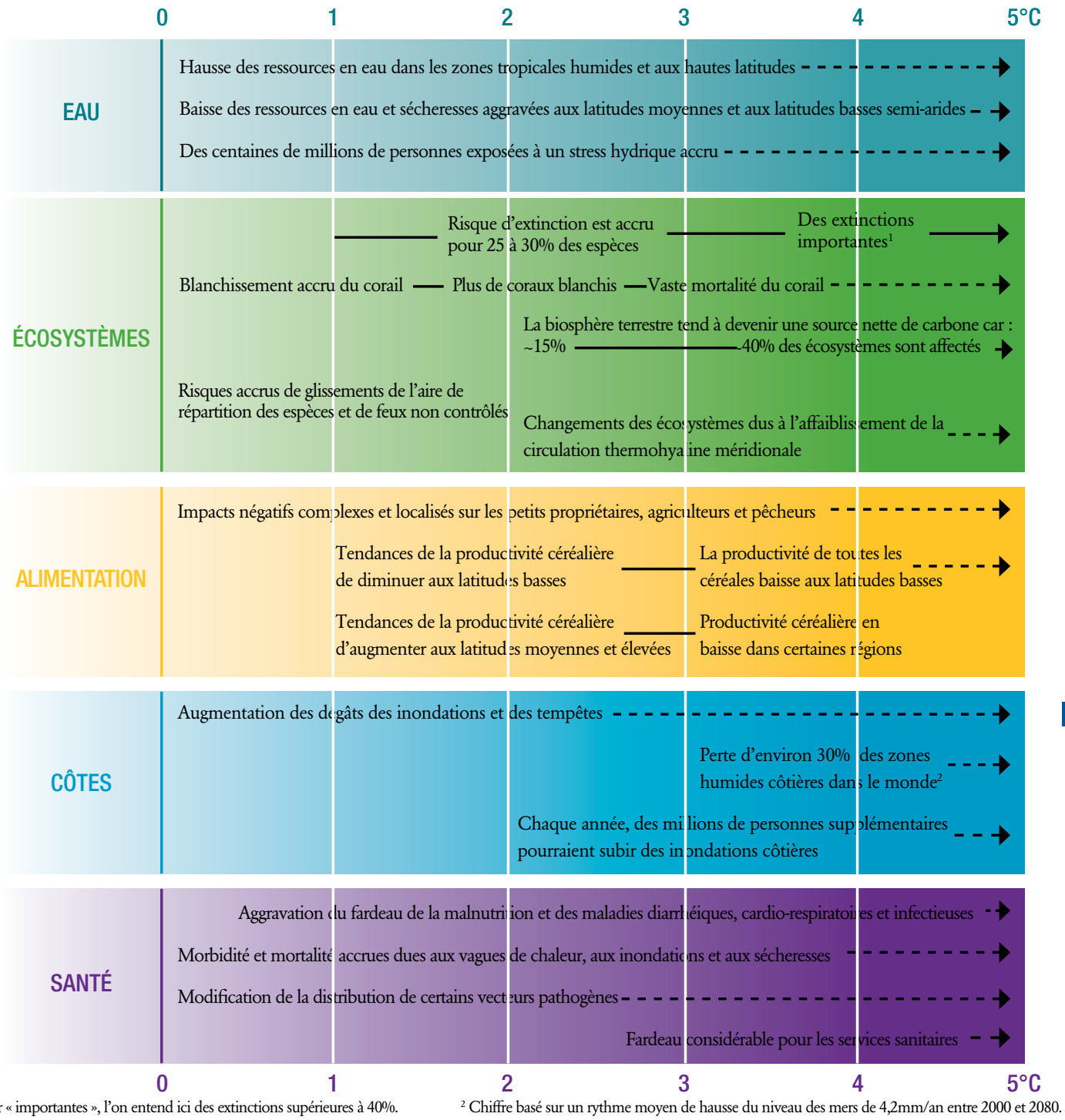

${ }^{1} \mathrm{Par}$ « importantes ", l'on entend ici des extinctions supérieures à 40\%. $\quad{ }^{2}$ Chiffre basé sur un rythme moyen de hausse du niveau des mers de 4,2mm/an entre 2000 et 2080.

Figure 5.1 Impacts de la hausse des températures (GIEC, 2007a)

(comme les récifs de corail). Les changements climatiques vont affecter la distribution, la démographie et la vie des espèces, avec des conséquences sur les moyens de subsistance humains, y compris des modifications des schémas de distribution des maladies (Encadré 5.2).

Les impacts des changements climatiques sur les espèces ne sont pas uniformément répartis dans tout le spectre de vie, qu'il soit taxonomique ou géographique. Les groupes d'espèces plus vulnérables incluent celles qui sont déjà rares et menacées : les espèces migratrices, celles dont l'aire de répartition est limitée (endémiques étroits), les communautés polaires, les populations périphériques, les espèces génétiquement appauvries et les espèces spécialisées, comme les espèces alpines et insulaires. Les espèces dont la spécialisation est maximale, au point de vue du mode de vie ou de 
la situation géographique, sont clairement les plus vulnérables. En se basant sur ces caractéristiques, la Commission de sauvegarde des espèces (CSE) a réalisé une évaluation des espèces sensibles aux changements climatiques et qui sont donc éventuellement en danger d'extinction (Vié et al., 2009). Elle note que :

- 35\% des oiseaux, $52 \%$ des amphibiens et $71 \%$ des coraux possèdent des caractéristiques qui les rendent particulièrement sensibles aux impacts des changements climatiques, et

- $70-80 \%$ des oiseaux, des amphibiens et des coraux qui sont déjà menacés sont aussi « sensibles aux changements climatiques ».

Si les changements climatiques ont un impact négatif sur certaines espèces, ils créent aussi des conditions favorables à d'autres. Les caractéristiques qui rendent des espèces envahissantes - la capacité de survivre dans des conditions difficiles, un taux de croissance rapide et une large dispersion - sont aussi celles qui vont les aider à prospérer avec les changements climatiques. Ensemble, les changements climatiques et les espèces envahissantes peuvent mettre de nombreuses espèces indigènes dans des situations qui dépassent leur capacité de s'adapter. Par exemple, en Californie, Mytilus galloprovincialis, une espèce de moule bleue envahissante, présente une tolérance à des températures d'eau plus élevées et à des taux de salinité accrus meilleure que celle de la moule bleue indigène, Mytilus trossulus. $M$. galloprovincialis a donc remplacé la moule indigène sur une bonne partie de la côte centre et sud de la Californie (Braby et Somero, 2006).

Les changements climatiques ont aussi des impacts au niveau des écosystèmes et l'on s'attend à ce que les écosystèmes polaires ainsi que ceux de type méditerranéen du Bassin de la Méditerranée, de Californie, du Chili, d’Afrique du Sud et de l'Ouest de l'Australie soient particulièrement affectés par les changements
Encadré 5.2 Quelques exemples d'impacts des changements climatiques sur des espèces

\section{Changements de la distribution des espèces}

- Poissons de mer (Perry et al., 2005)

- Plantes de montagne du sud de la Californie (Kelly et Golden, 2008)

- Papillons (Warren et al., 2001)

- Amphibiens et oiseaux tropicaux (Pounds et al., 1999)

- Oiseaux de Grande-Bretagne (Thomas \& Lennon, 1999)

- Ours polaires (Derocher et al., 2004)

- Distribution des arbres en ColombieBritannique (Hamann et Wang, 2006)

\section{Changements de démographie de population}

- Changements possibles chez les espèces où la détermination du sexe dépend de la température (Schwanz et Janzen, 2008)

\section{Changements du comportement des espèces}

- Envol plus précoce des insectes (Ellis et al., 1997 ; Woiwod, 1997)

- Nidification plus précoce des oiseaux, reproduction plus précoce des amphibiens et floraison précoce des arbres (Walther, 2002)

- Saisons de frai sautées chez les harengs (Engelhard et Heino, 2006)

- Capacité de certains coquillages de fixer le calcium (Gazeau et al., 2007)

climatiques (Lavorel, 1998 ; Sala et al., 2000). La mise à jour 2008 de l'État des Récifs coralliens du monde comprenait de bonnes et de mauvaises nouvelles. Les récifs de l'ouest du Pacifique et de l'océan Indien, qui avaient souffert précédemment d'un grave blanchissement suite à la montée de la température des océans, sont en train de récupérer mais les impacts généraux 
des changements climatiques et de l'activité humaine restent des menaces importantes pour la survie à long terme des récifs du monde (Wilkinson, 2008). Les écosystèmes de haute altitude et les forêts brumeuses sont aussi en danger. Dans l'est de l'Amazonie, on s'attend à ce que la savane remplace la forêt tropicale ainsi qu'à d'autres changements de végétation dans le nord-est du Brésil, suite aux effets synergiques de l'aménagement du territoire et des changements climatiques (Magrin et al., 2007).

Les changements climatiques vont certainement modifier la façon dont la biodiversité est gérée et préservée. Burns et al. (2003) ont étudié l'impact des changements climatiques sur la diversité des mammiferes dans les parcs nationaux américains et ils ont noté que, à cause de pertes d'espèces allant jusqu'à $20 \%$ et de l'afflux rapide de nouvelles espèces, les aires protégées pourraient ne plus être à même de remplir leur mandat initial en termes de conservation. Les organes de gestion devront déterminer comment concevoir et gérer au mieux les aires protégées aujourd'hui pour qu'elles soient capables de s'adapter aux éventuels changements climatiques et d'aider à en atténuer les causes (par exemple, en stockant du carbone dans la végétation, les sols et les eaux). Le Comité du Patrimoine mondial de l'UNESCO reconnaît que les changements climatiques affectent déjà des aires protégées dans le monde entier et qu'ils vont probablement en toucher beaucoup plus dans les années à venir. En réponse, il a adopté une stratégie pour aider les pays à s'occuper de cette menace (UNESCO, 2006).

Avec des impacts déjà évidents au niveau aussi bien des espèces que des écosystèmes, la fourniture des services écosystémiques va inévitablement être affectée aussi. Par exemple, des scientifiques prévoient une modification du régime des pluies dans le sud de l'Afrique suite aux changements de température dans l'océan Indien, avec pour résultat des baisses de production agricole et une augmentation de l'insécurité alimentaire dans la région (Funk $e t$ al., 2008). D’autre part, les écosystèmes peuvent jouer un rôle en atténuant ces impacts. Sheil et Murdiyarso (2009) notent le rôle éventuel que pourraient jouer les forêts dans les cycles hydrologiques et le maintien des chutes de pluies. Ces résultats, et d'autres similaires, constituent des arguments solides pour étayer les approches par paysage de l'adaptation et de l'atténuation des changements climatiques.

\section{LES DÉFIS PARTICULIERS AUX ÎLES}

Les petits états insulaires sont particulièrement vulnérables face à la plupart des changements climatiques. Leur superficie diminue quand le niveau de la mer monte, les récifs qui les protègent diminuent lorsque les océans s'acidifient, et leur couverture végétale a des difficultés à s'adapter aux nouveaux climats parce qu'elle n'a qu'un faible degré de diversité. De nombreuses îles souffrent déjà d'une dégradation environnementale suite à la pollution, à la destruction de l'habitat, à l'invasion d'espèces exotiques et à la surexploitation. Lorsque s'ajoutent les impacts des changements climatiques, on peut s'attendre à ce que seules les espèces les plus robustes (souvent des espèces envahissantes) survivent.

Il faut adopter une approche à l'échelle du paysage pour aider les îles dans leurs efforts pour s'adapter aux impacts des changements climatiques. Lorsque des forêts et des zones humides intérieures sont liées aux écosystèmes côtiers, il faudrait les gérer comme une entité intégrée. Différentes catégories d'aires protégées peuvent constituer une partie importante de la mosaïque du paysage, qui peut aider les écosystèmes à s'adapter aux impacts des changements climatiques.

Les îles sont aussi d'excellents laboratoires où l'on peut étudier les impacts des changements 
climatiques et les réponses pour s'y adapter ou les atténuer. L'UICN, par son Initiative Mangroves pour l'avenir, travaille avec d'autres organisations pour gérer durablement des écosystèmes côtiers, de façon à améliorer leur capacité de s'adapter et d'augmenter la résilience des communautés qui en dépendent.

\section{ATTÉNUATION, ADAPTATION ET BIODIVERSITÉ}

L'atténuation des changements climatiques vise à éviter l'ingérable; l'adaptation aux changements climatiques vise à gérer l'inévitable. Les réponses, en termes d'atténuation et d'adaptation, doivent être développées sur la base d'une meilleure compréhension du contexte dynamique et des effets en cascade de toutes les menaces et de leurs impacts possibles auprès de toutes les parties prenantes. Fin 2006, le Gouvernement britannique a publié un rapport détaillé sur l'économie des changements climatiques (Encadré 5.3) qui concluait que les mesures pour stopper les changements climatiques étaient économiquement très raisonnables. Le rapport met spécifiquement en évidence la conservation des forêts comme moyen très rentable de ralentir les changements climatiques, donnant ainsi des arguments - pour des raisons de climat et de conservation - pour éviter la déforestation (Stern, 2006).

Les Membres de l'UICN, par la Résolution CMN 4.075 entre autres, ont exprimé les liens très forts entre conservation, biodiversité, et action en faveur du climat. Cette action reconnaît le rôle des écosystèmes, y compris les forêts, les tourbières, les prairies, les bassins hydrographiques et les écosystèmes côtiers dans le soutien à la mitigation et à l'adaptation aux changements climatiques. En termes de politique internationale en matière de changements climatiques dans la CCNUCC, l'UICN s'est penchée tout particulièrement sur deux opportunités clés pour la biodiversité - la Réduction des émissions issues de la déforestation et de la dégradation des forêts dans les pays en développement (REDD) et l'Adaptation écosystémique (EbA).

\section{REDD}

En termes de mitigation, la REDD apporte de réelles opportunités pour atténuer les impacts des changements climatiques en préservant aussi les ressources forestières mondiales, les services écosystémiques associés et les moyens de subsistance qui en dépendent. La déforestation et la dégradation des écosystèmes forestiers sont des causes importantes du réchauffement mondial enregistré au siècle passé, comptant pour 17\% des émissions de GES mondiales, soit plus que l'ensemble du secteur des transports. La Eliasch Revieze du Gouvernement britannique (2008) soutient que « sans compenser la perte de forêts, il est très peu probable que nous pourrons atteindre une stabilisation des concentrations de gaz à effet de serre dans l'atmosphère à un niveau qui puisse éviter les pires effets des changements climatiques ".

Une autre ressource que le monde peut examiner, ce sont les stocks de carbone sur pied (forêts) dans les aires protégées du monde entier - on a calculé qu'ils représentaient 312 Gt de carbone, soit $15 \%$ du tout le carbone séquestré dans le monde (CDB, 2008).

Mais un mécanisme REDD dans la CCNUCC qui ne tiendrait pas compte des avis des personnes les plus directement concernées serait condamné d'avance. Un mécanisme REDD réussi exige que l'on fasse attention aux problèmes de gouvernance s'il ne veut pas s'aliéner les communautés locales qui dépendent de la forêt ni les priver de leur voix, ce qui susciterait des conflits et compromettrait la possibilité d'assurer des réductions d'émissions permanentes et supplémentaires. Les pays peuvent s'inspirer d'un ensemble important d'accords internationaux et des directives de bonne pratique pour établir, 
Encadré 5.3 Messages clés du rapport

Stern sur les changements climatiques

Laisser les changements climatiques

progresser selon un scénario business-as-usual

va entraîner un coût situé entre 5\% et $20 \%$

du PIB mondial, alors que passer à une

économie lore-carbon qui stabiliserait le climat

coûterait environ 1\% du PIB mondial. En

bref, le rapport trouve que les bénéfices

d'une stabilisation du climat dépassent de loin son coût.

Les impacts des changements climatiques vont beaucoup varier selon les pays et, comme dans les analyses précédentes, il appert que ce sont les pays en développement qui seront probablement les plus affectés.

Source: Stern, 2006

au niveau national, les fondements nécessaires aux mécanismes REDD. Citons, par exemple, l'Instrument juridiquement non contraignant des NU sur tous les types de forêts ; le Programme de travail étendu sur la biodiversité forestière de la Convention sur la diversité biologique et la Déclaration des Nations Unies sur les droits des populations autochtones, entre autres.

\section{ADAPTATION ÉCOSYSTÉMIQUE (EbA)}

Les services écosystémiques (Chapitre 4) soustendent le bien-être humain, et certains sont essentiels pour réguler le climat et gérer les impacts des changements climatiques. D'autre part, l'évolution vers des schémas climatiques qui sont inévitables va compromettre certains des services que fournissent les écosystèmes et donc menacer les moyens de subsistance des hommes. La hausse du niveau des mers, les violentes tempêtes, la fonte des glaciers, les sécheresses et les inondations induites par le réchauffement global vont faire que des millions de personnes vont perdre leurs maisons et leurs moyens de subsistance, et parfois même leur vie.

Les preuves laissent penser qu'un système diversifié sera plus résilient s'il est confronté à un changement environnemental et qu'il manifestera donc une plus grande adaptabilité de son écosystème. En soi, une plus grande diversité d'espèces et de populations remplissant des fonctions comparables dans un écosystème a une plus grande probabilité de permettre la préservation des processus écosystémiques en cas de changement environnemental (McCann, 2000). L'EbA identifie et met en œuvre toute une gamme de stratégies pour la gestion, la conservation et la restauration d'écosystèmes, pour garantir qu'ils continuent à fournir les services qui permettent aux hommes de s'adapter aux impacts des changements climatiques ${ }^{2}$. Une EbA est une solution d'adaptation rentable, immédiate et accessible. Parmi les exemples d'EbA, citons la gestion d'écosystèmes côtiers pour réduire les inondations en cas de tempêtes. Les mangroves, les marais salants, et d'autres types de végétation côtière constituent des infrastructures naturelles qui réduisent l'impact de l'énergie des vagues à l'intérieur des terres, qui agissent comme des barrières contre les débris et réduisent l'érosion côtière. Citons encore la préservation ou l'amélioration de la résilience des écosystèmes à l'échelle des paysages, grâce à des systèmes d'aires protégées gérés efficacement et à des améliorations de la gestion des terres ou des mers environnantes.

Les impacts des changements climatiques sur les hommes seront répartis différemment selon les régions, les générations, les classes d'âge, les tranches de revenus, les occupations et les sexes. Les communautés rurales, qui sont souvent

${ }^{2}$ Cette définition provient, en parfaite cohérence, du rapport provisoire du Groupe ad-hoc d'experts techniques de la Convention sur la diversité biologique sur les changements climatiques et la biodiversité. 
celles qui dépendent le plus directement des ressources naturelles locales, seront les plus affectées, spécialement celles qui vivent dans les environnements les plus vulnérables. Les pauvres, d'abord mais pas exclusivement ceux des pays en développement, seront affectés de manière disproportionnée en raison de leur capacité réduite de s'adapter aux changements et de leur dépendance vis-à-vis de ressources comme l'eau et la nourriture qui seront touchées par les changements climatiques (GIEC, 2007b). Par contre, ces groupes peuvent aussi être de puissants vecteurs de changement en jouant un rôle majeur dans la réduction des désastres, dans la déforestation et les utilisations d'énergie. De plus, une EbA est un moyen d'adaptation souvent plus accessible pour les pauvres des zones rurales que les solutions technologiques ou infrastructurelles. Une EbA peut être un moyen d'aider les populations autochtones à s'adapter aux conditions climatiques changeantes, si l'on tire profit des savoirs traditionnels qui intègrent aussi des besoins spécifiques à chaque sexe en matière de ressources naturelles.

Les impacts des changements climatiques peuvent mettre en danger les stratégies de réduction de la pauvreté et miner les efforts pour atteindre l'ensemble des Objectifs du millénaire pour le développement (OMD). C’est pourquoi les stratégies de réduction de la pauvreté et la planification de tout développement doivent intégrer une $\mathrm{EbA}$, comme un élément intégral des programmes généraux visant à réduire la pauvreté et à s'adapter aux changements climatiques. De nombreux instruments sont disponibles pour aider les communautés locales à identifier la nature de leur fragilité face aux impacts des changements climatiques, et aussi les options dont elles disposent pour s'y adapter à l'avenir. Parmi ceux-ci, CRiSTAL (www.cristal.org), un outil d'identification des risques mis au point par l'Institut international du développement durable (IIDD), l'UICN, Intercoopération et l'Institut de Stockholm pour l'environnement (SEI), aide les planificateurs du développement et de la conservation à travailler avec les communautés locales pour intégrer dans leurs projets les besoins en matière d'adaptation aux changements climatiques.

\section{AUTRES CONSIDÉRATIONS}

En gérant les impacts des changements climatiques, nous ne pouvons pas ignorer l'impact des choix en matière d'énergie aussi bien sur la planification que sur la mise en œuvre des mesures d'atténuation et d'adaptation. Ceci sera discuté en détail au chapitre 7. De plus, les questions relatives aux changements climatiques doivent inclure comme démarche complémentaire certains aspects d'approches basées sur les droits, les synergies entre divers instruments internationaux concernant les changements climatiques, et la réduction des risques de catastrophes (Chapitre 10).

La Déclaration de Stockholm (1972) affirmait que l'homme a « un droit fondamental à la liberté, à l'égalité et à des conditions de vie satisfaisantes, dans un environnement dont la qualité lui permettra de vivre dans la dignité et le bien-être ". L'impact des changements climatiques va mettre en danger nombre des éléments fondamentaux qui sous-tendent ces " conditions de vie satisfaisantes » et l'on peut donc considérer qu'ils affectent les droits de l'homme. C'est pourquoi toute mesure prise en termes d'adaptation devrait inclure une attention pour les droits humains comme un élément essentiel. En avril 2009, le Bureau du Haut Commissaire des Nations Unies pour les droits de l'homme, à la demande du Conseil des droits de l'homme des Nations Unies, a publié un rapport sur la relation entre changements climatiques et droits de l'homme (NU, 2009).

La gouvernance de problèmes liés aux changements climatiques ne relève pas de la seule compétence de la CCNUCC et 
des gouvernements Parties. Les liens entre changements climatiques et biodiversité exigent des actions dans d'autres accords internationaux pertinents (McNeely, 2008). La Convention sur la diversité biologique, par exemple, discute du rôle de la biodiversité aussi bien pour la séquestration de carbone que pour l'adaptation aux changements climatiques, alors que les Conventions sur les zones humides (Ramsar) et la désertification (CCD) traitent des habitats dont la gestion efficace contribuera à l'adaptation aux changements climatiques dans les prochaines décennies. La Convention des Nations Unies sur le droit de la mer (CNUDM - UNCLOS) s'occupe spécifiquement des écosystèmes marins, et de récentes recherches ont montré combien les environnements marins sont importants lorsqu'il s'agit des problèmes impliquant les changements climatiques. La Convention sur les espèces migratrices (CMS) s'occupe d'espèces dont l'aire de répartition est très vaste et qui sont susceptibles d'être influencées par les changements climatiques et elle devrait donc appuyer le processus d'adaptation. Etant donné que les ressources et le temps sont comptés, un point critique sera de se concentrer sur une coordination d'action plus efficace de tous ces instruments et de prendre des mesures strictes de mise en ouvre et d'application.

L'adaptation aux changements climatiques et la réduction des risques de catastrophes sont des approches complémentaires. Par le Cadre d'action de Hyogo pour 2005-2015, des pays se sont engagés à intégrer l'adaptation aux changements climatiques et la réduction des risques de catastrophes, grâce à l'identification des risques de catastrophes liés au climat, à la conception de mesures spécifiques pour réduire ces risques, et à l'utilisation meilleure et plus systématique des informations sur les risques climatiques par leurs planificateurs, ingénieurs et autres décideurs.
Les changements climatiques sont le premier vecteur de changement du monde d'aujourd'hui et une menace croissante pour la biodiversité et les services écosystémiques qu'elle produit. Alors que le monde entier se réunit à Copenhague pour discuter et adopter un cadre post-2012 pour les changements climatiques, il est vital que les Etats qui négocient à la CCNUCC :

- Intègrent les questions de biodiversité dans tous les efforts pour atténuer les changements climatiques et s'adapter aux changements inévitables, par exemple par la Réduction des émissions issues de la déforestation et de la dégradation des forêts (REDD) et par des adaptations fondées sur les écosystèmes ;

- Intègrent des approches basées sur le sexe et sur les droits ; et

- Tiennent compte du travail effectué par le Cadre d'action de Hyogo lors de la conception et de la mise en œuvre des plans et des stratégies d'adaptation.

Mais la tâche n'est pas du seul ressort des gouvernements. A l'issue de la crise financière, les initiatives pour renouveler l'économie mondiale devraient profiter de l'occasion de "décarboner " cette économie en développant des incitants novateurs, comme des marchés de carbone, qui fourniront de nouveaux modèles, plus diversifiés, de paiement pour des services écosystémiques. Les prix payés pour des biens et services doivent comprendre le " coût social du carbone " pour refléter l'impact sur les changements climatiques de tout le processus qui va de la fourniture de la matière brute à sa consommation, en passant par sa production et sa distribution. Cela doit s'appliquer à tous les pays, y compris pour les produits exportés. Certains ont suggéré que les pays prélèvent un "droit carbone » sur les importations pour refléter toute émission de GES associée à leur production. Les taxes carbone à l'importation peuvent ne pas être du goût 
de certains, mais on peut arguer avec la même conviction qu'elles sont essentielles pour la survie de la planète.

Les aires protégées ont déjà montré leur valeur pour la conservation d'une biodiversité qui, sans cela, pourrait être perdue. Lorsqu'elles sont correctement conçues et gérées, les aires protégées pourraient aussi permettre d'atténuer les changements climatiques et de s'y adapter. La préservation du couvert forestier dans des aires protégées peut être une contribution utile à la REDD, pour autant que l'on puisse prouver qu'il s'agit bien d'une " additionalité ", et la gestion à l'échelle du paysage d'écosystèmes qui comprennent des aires protégées sera un aspect important de la planification de l'adaptation aux changements climatiques.

Enfin, et ce n'est pas le moins important, les efforts pour atténuer les changements climatiques doivent s'assurer que des stratégies énergétiques alternatives, y compris l'option de l'utilisation de biocarburants, prennent complètement en compte - et protègent contre - tout impact négatif sur le climat, la biodiversité et les moyens de subsistance. 


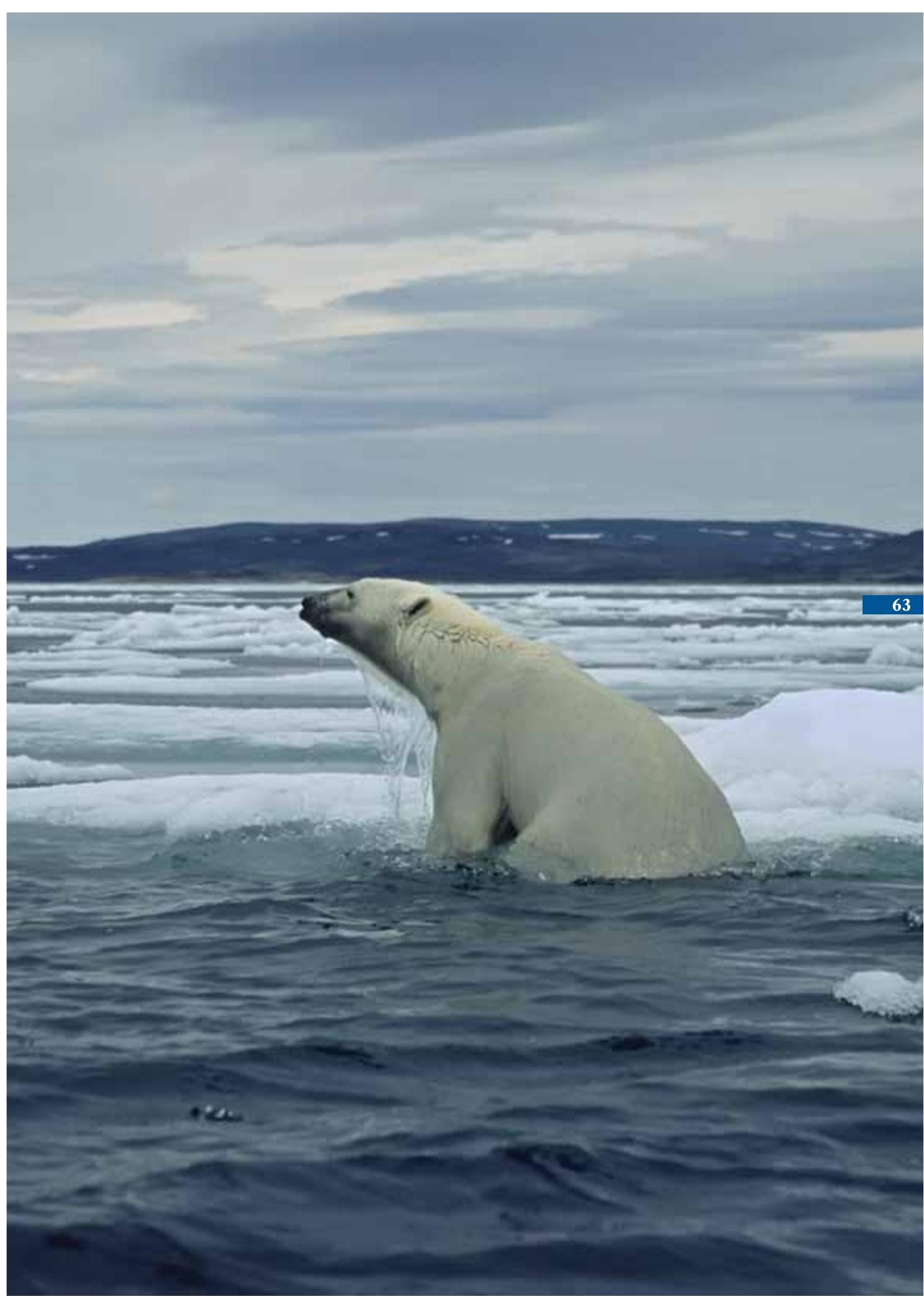




\section{6. \\ Aires protégées : pour l'amour de la vie}

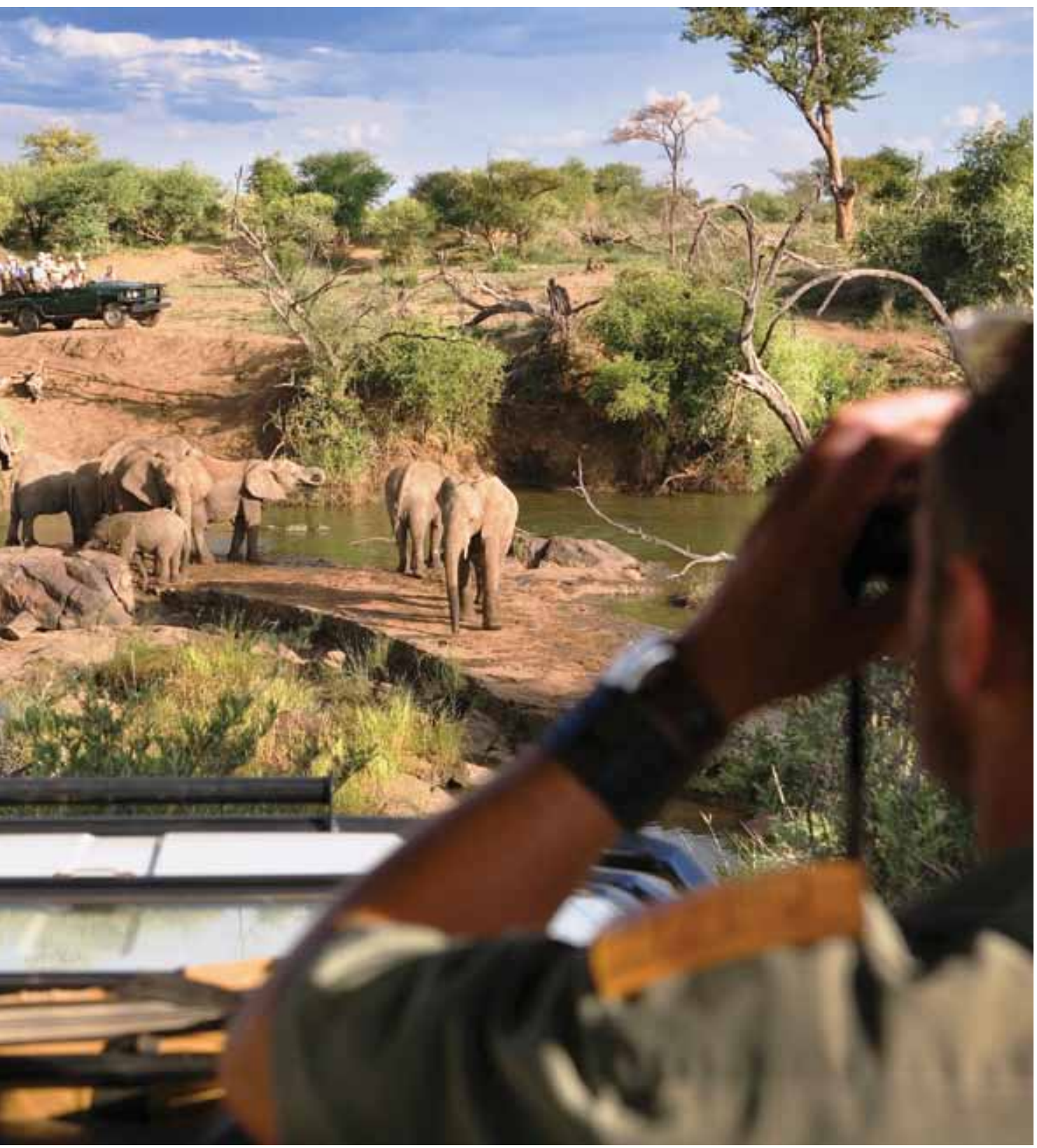




\section{En 2003, le monde de la conservation s'est réuni à Durban pour le Vº Congrès mondial des parcs et il a adopté l'Accord de Durban comme nouveau paradigme pour l'avenir (UICN, 2003). L'Accord reconnaît le rôle critique des aires protégées (AP) qui supportent la conservation de la biodiversité ainsi que les efforts pour réduire la pauvreté, le support au développement économique et la promotion de la paix. II a applaudi le triplement du nombre d'aires protégées au cours des 20 années écoulées}

L'Accord a pourtant aussi exprimé des inquiétudes. Si 11,5\% de la surface terrestre bénéficiaient de l'une ou l'autre forme de conservation, le réseau actuel ne représentait pas encore la gamme complète de la biodiversité, spécialement dans le milieu marin. Les coûts et bénéfices des aires protégées n'étaient pas répartis équitablement et les financements destinés à ces aires étaient lamentablement inadéquats. Par conséquent, la gestion de nombreux sites était compromise au point que "de nombreux parcs existaient plus sur papier qu'en réalité ». Le rôle historique des communautés locales, des populations autochtones et nomades dans la conservation de la biodiversité et la valeur des aires protégées pour ces groupes étaient rarement reconnus ou inclus dans les planifications et les mises en œuvre. Les changements, et particulièrement les changements climatiques, compliquaient les défis existants des aires protégées du monde entier.

Les succès et les préoccupations relevés dans l'Accord de Durban sont certainement encore d'actualité. Par exemple, 13 des 199 Sites naturels du patrimoine mondial sont classés " en danger», même des sites importants pour la biodiversité comme les Galápagos, le Parc National de Manas, en Inde, et cinq parcs nationaux de la République Démocratique du Congo, pour des raisons liées à des guerres civiles, à l'impact du tourisme et à des espèces envahissantes (UNESCO, 2009, chiffres de mai 2009). Le nombre de Sites du patrimoine mondial qui sont en danger critique n'est pas totalement représenté dans la Liste des patrimoines en danger de l'UNESCO (Badman et al., 2009).

Les discussions de Barcelone ont examiné les progrès accomplis dans beaucoup de ces domaines et elles sont reprises dans plusieurs chapitres de ce volume. Le rôle des populations locales et des aires protégées est traité au chapitre 3 , les changements climatiques et les aires protégées au chapitre 5 , les aires protégées, les conflits et les parcs pour la paix au chapitre 9 et les aires marines protégées au chapitre 17. Ici, ce sont les défis en matière de représentativité, d'efficacité de gestion et de financement pour les aires protégées qui sont analysés en profondeur. 


\section{UN RÉSEAU D'AIRES PROTÉGÉES REPRÉSENTATIF}

Même avec 11,5\% de la terre retenus comme aires protégées, il reste d'importantes lacunes. Rodrigues et al. (2004) ont souligné les défis qui consistent à déterminer des regroupements de cibles mondiales comme moyens d'établir des réseaux d'aires protégées représentatifs et ils identifiaient plusieurs lacunes dans le réseau existant. Ils notaient que sur les espèces considérées, au moins 12\% ne sont représentées dans aucune aire protégée et que d'autres taxons comptant un fort taux d'endémisme, comme les plantes et les insectes, sont encore moins bien représentés, étant donné la tendance de certains ensembles d'espèces ayant de plus petites aires de répartition d'avoir de plus fortes proportions d'« espèces lacunaires ».

Il faut des outils pour identifier les lacunes plus en détail et faire connaître ce problème. Un effort important en ce sens fut le lancement de la Banque de données mondiale 2008 sur les aires protégées (la mise à jour 2009 est disponible sur www.wdpa.org ), un outil en ligne nettement amélioré qui permet aux utilisateurs de zoomer, de survoler et d'explorer plus de 100.000 parcs nationaux.

Un autre pas dans cette direction fut le lancement de Google Ocean, un effort conjoint de l'UICN, de Google, et d'autres partenaires, focalisé sur le réseau actuel d'aires marines protégées (http:// earth.google.fr/intl/fr/ocean/). D'autres outils disponibles comprennent le développement de lignes directrices pour aider à l'identification, à la priorisation et à l'analyse des lacunes des Zones clés pour la biodiversité (KBA), des sites d'importance mondiale pour la conservation de la biodiversité, identifiés en se servant de critères et de seuils standardisés au niveau mondial (Eken et al., 2004). Connaissant les KBA, les gestionnaires d'aires protégées peuvent alors entreprendre une analyse des lacunes et œuvrer vers des réseaux d'aires protégées plus complets pour préserver la biodiversité (Langhammer et al., 2007).

Chaque pays devrait préparer l'analyse de son propre système d'aires protégées pour s'assurer que tous les habitats clés et les formations naturelles sont protégés par une catégorie de gestion adéquate. Il faut accorder une attention particulière aux aires marines protégées (AMP) (Chapitre 17). 5000 AMP couvrent aujourd'hui plus de 2,35 millions de kilomètres carrés, mais cela ne représente que $1,6 \%$ de la surface marine totale au sein des Zones économiques exclusives. La Commission mondiale des aires protégées (CMAP) de l'UICN et le Programme Marin travaillent avec de nombreux autres partenaires pour arriver à protéger $10-30 \%$ des habitats marins d'ici 2012 (Laffoley, 2008).

\section{GESTION EFFICACE DES AIRES PROTÉGÉES}

Les questions d'efficacité de gestion discutées à Barcelone comprenaient la disponibilité d'outils et des lignes directrices, l'engagement des communautés locales, les espèces envahissantes et le besoin d'une approche de la gestion des aires protégées à l'échelle du paysage.

Depuis Durban, la CMAP a investi des efforts considérables dans le développement d'outils et de lignes directrices pour aider à l'amélioration de l'efficacité de la gestion du réseau existant d'aires protégées. Hockings et al. (2006) ont produit un cadre pour évaluer l'efficacité. A Barcelone, les Lignes directrices pour l'application des catégories de gestion aux aires protégées (Dudley, 2008) furent publiées, insistant sur le fait que, si l'objectif prioritaire pour toutes les aires protégées est de protéger la nature, les aires protégées ont d'autres objectifs importants qui visent à améliorer les moyens de subsistance des hommes. Les besoins des peuples qui vivent dans et autour des aires protégées doivent recevoir une attention 
beaucoup plus soutenue. Redford et Fearn (2007) examinent les compromis, les conflits, les flux de bénéfices et de coûts, les questions juridiques et les nombreux autres domaines qui doivent être analysés maintenant que la gestion d'une aire protégée devient plus démocratique. Coad et al. (2008), qui ont aussi analysé les coûts et bénéfices des aires protégées pour les communautés locales, ont noté que les impacts des aires protégées sur les moyens de subsistance varient en fonction du statut de l'aire protégée, des stratégies de gestion et de l'implication de la communauté dans la gouvernance. La plupart des coûts étaient liés à des aires protégées dont les structures de gestion sont de nature top-

down (généralement associées aux catégories de gestion I-II de l'UICN) ou à des aires protégées où les capacités de gestion et institutionnelles font défaut et où les problèmes de gouvernance et de droit foncier ne sont pas résolus. D'autre part, les schémas de gestion communautaires et la gestion des aires protégées qui permet l'utilisation durable des ressources (plus souvent associées aux catégories de gestion V-VI de l'UICN) peuvent engendrer des bénéfices tangibles. BorriniFeyerabend et al. (2004) discutent les mécanismes permettant d'accroître la conservation et l'équité des communautés locales et autochtones dans les aires protégées.

La Convention sur la diversité biologique (CDB), de nombreux gouvernements et des organisations de conservation reconnaissent les droits, les compétences et les connaissances des communautés locales et autochtones, et ils accordent une attention spéciale et du respect aux sites naturels sacrés. Cette dimension culturelle de certaines aires protégées mérite une meilleure attention ces prochaines années et elle devrait être incluse dans l'examen de la représentativité de ces aires protégées. L'UICN et l'UNESCO ont développé des Lignes directrices pour les sites naturels sacrés à l'intention des gestionnaires d'aires protégées (en anglais), qui reconnaissent l'importance des valeurs culturelles et spirituelles dans la conservation de la nature et fournissent des orientations pratiques pour la gestion de ces valeurs dans des aires protégées (Wild et McLeod, 2008). Les membres de l'UICN ont renforcé l'importance des sites sacrés dans les aires protégées par la Résolution $4.038 \mathrm{du}$ CMN.

Dans pratiquement toutes les parties du monde, une menace majeure pour les aires protégées est l'invasion d'espèces non indigènes, allant des chèvres aux Galápagos à la jacinthe d'eau dans les Grands Lacs africains. Certains gestionnaires d'aires protégées ont même introduit volontairement des espèces exotiques envahissantes dans leur parc, comme la truite dans certaines aires protégées de montagne des États-Unis, croyant que cela rendrait leurs cours d'eau plus attractifs pour les visiteurs, même si certaines espèces natives devaient s'en trouver menacées. Il faut faire des efforts bien plus grands pour empêcher les espèces non indigènes d'envahir des aires protégées, pour les éradiquer dès que possible si elles envahissent et pour minimiser leurs impacts si elles finissent quand même par s'installer (McNeely et al., 2001). De plus, les stratégies pour éradiquer les espèces envahissantes des aires protégées devraient, autant que possible, inclure la gestion du risque d'effets secondaires indirects (Bergstrom et al., 2009).

Dans tout scénario réaliste pour l'avenir, les aires protégées seules seront insuffisantes pour réellement préserver la biodiversité de la planète, 
à moins que les terres et l'eau situées en dehors du système d'aires protégées soient gérées de façon compatible avec les objectifs de la conservation des aires protégées. Les aires protégées ne peuvent plus être des îles d'habitats naturels dans une mer d'utilisations incompatibles du territoire, moins encore des forteresses contre les intérêts humains locaux. Au contraire, il faut considérer les aires protégées comme faisant partie des paysages régionaux, interconnectées par des habitats corridors qui étendent le territoire réel des espèces ayant une vaste aire de répartition ; elles contribuent aux services écosystémiques rendus aux populations locales et soutiennent l'adaptation à des conditions changeantes. Pour y arriver, une conservation réussie exigera de travailler à une échelle plus large, qui intégrera le niveau du paysage terrestre et marin, étant donné que les défis auxquels font face les aires protégées sont trop complexes et impliquent trop de groupes d'intérêt pour être résolus au niveau de chaque site.

Les Membres de l'UICN ont reconnu le besoin de connectivité dans la Résolution 4.062, appelant à une plus grande attention pour la connectivité dans les initiatives de conservation à grande échelle, y compris des liens entre les aires protégées dans un paysage élargi. Les exemples d'une telle approche par paysage comprennent le Corridor du Yellowstone au Yukon, le Corridor biologique méso-américain, la Ceinture verte européenne et l'Arc du Terai entre l'Inde et le Népal. Traiter de tels problèmes sera plus facile si l'opinion publique est favorable aux aires protégées et, comme plus de gens vivent en ville, il faudra trouver des façons novatrices pour sensibiliser la population urbanisée.

En appui à l'efficacité de gestion, la CMAP a maintenant publié 16 Lignes directrices sur les meilleures pratiques comprenant, en plus de celles déjà mentionnées, des questions comme les lignes directrices sur la planification de la gestion (Thomas et Middleton, 2003) et les aires protégées transfrontalières (Sandwith et al., 2001). La liste complète se trouve sur http:// www.iucn.org/about/union/commissions/wcpa/ wcpa_puball/wcpa_bpg/. La formation devient aussi accessible sur Internet grâce à des outils comme le Réseau d'apprentissage sur les aires protégées (PALNet) et les activités soutenues par les membres de l'UICN et par ses partenaires.

\section{FINANCEMENT ET CAPACITÉS ADÉQUATS POUR LES AIRES PROTÉGÉES}

Bien que des fonds substantiels soient mis à la disposition des aires protégées, cela n'a pas été suffisant pour suivre leur extension de ces dernières années (Emerton et al., 2006). Une grande partie des financements pour les aires protégées s'est faite à court terme et concentrée sur des investissements de capitaux, avec un soutien très limité dans le temps pour les structures et les institutions des aires protégées. Cela a laissé beaucoup d'aires protégées sous-financées et susceptibles de le rester dans les conditions actuelles. Bruner et al. (2004) ont estimé que le financement manquant pour gérer les aires protégées existantes dans les pays en développement était d'environ 1,3 milliard de dollars, ajoutant ironiquement que ce montant correspondait à 2\% de ce que les Américains dépensent par an en boissons non alcoolisées (Jacobsen, 2004). D’autres estimations des lacunes dans le financement des aires protégées sont beaucoup plus élevées (ex. jusqu'à 45 milliards de dollars par an pendant 30 ans pour sécuriser et étendre les aires protégées marines et terrestres, selon l'estimation de Balmford et al., 2002). Quels que soient les chiffres, il est clair qu'atteindre un financement durable va exiger le renforcement des capacités dans la communauté des aires protégées en matière de planification financière et d'affaires, ainsi qu'une politique et des conditions de marché favorables.

Comme le souligne déjà l'Accord de Durban, si les aires protégées doivent survivre face à des demandes croissantes, elles vont avoir besoin d'un 
support financier important. Cela ne devrait pas toujours être difficile, vu les bénéfices que les aires protégées génèrent. Par exemple, en 2003, quelque 266 millions de gens ont visité les parcs nationaux américains et dépensé environ 10 milliards de dollars pendant leur visite. Le tourisme concernant les parcs nationaux a généré 4,5 milliards de dollars en salaires et bénéfices divers, et a fourni 267.000 emplois. Garantir que les aires protégées reçoivent une part correcte des bénéfices qu'ils génèrent est un défi qui mérite une réflexion créative, mais il est probable que cela réussit mieux si l'on se base davantage sur le principe de l'utilisateur-payeur, notamment sur une hausse du droit d'entrée dans les parcs ou du prix des concessions accordées à des tour-opérateurs. De plus, les gouvernements pourraient devoir adopter le concept de l'utilisateurpayeur plus volontiers, en permettant aux aires protégées de conserver une plus grande part des revenus qu'elles génèrent. Par exemple, le Parc National de Serengeti, en Tanzanie, récolte des droits d'entrée à hauteur de 6 millions de dollars par an, ce qui est suffisant pour financer un niveau de gestion adéquat.

Néanmoins, certaines aires protégées peuvent difficilement générer assez de revenus seules, parce qu'elles sont peu attractives. Elles doivent donc continuer à dépendre de financements publics ou d'autres voies novatrices comme la perception de compensations pour les services écosystémiques qu'elles fournissent à la société au sens large. Les aires protégées doivent se considérer elles-mêmes comme des fournisseurs de services pour la société, offrant des services générateurs de revenus (loisirs, tourisme, fonctions écosystémiques) ou non (conservation de la biodiversité, éducation, valeurs culturelles). En ce qui concerne les services culturels, les gouvernements devraient considérer tout investissement dans les aires protégées de la même façon que ceux faits dans l'éducation et les arts - un moyen primordial pour soutenir la société et la créativité et les valeurs qui la sous-tendent.

\section{APRÈS BARCELONE}

Les années qui viennent vont donner

d'importantes opportunités aux aires protégées, en termes de gouvernance et d'engagement des jeunes dans le travail dans les aires protégées.

En termes de gouvernance environnementale internationale, la structure des conventions internationales - dont la CDB, la Convention du patrimoine mondial, la Convention relative aux zones humides d'importance internationale (Ramsar), la Convention des Nations Unies sur le droit de la mer (CNUDM - UNCLOS) - qui supportent la conservation par les aires protégées montre déjà son intérêt, mais on peut certainement faire beaucoup plus, notamment soutenir la gouvernance des aires protégées en haute mer, les aires protégées transfrontalières, et une meilleure coopération dans l'échange d'informations et le renforcement des capacités (UICN, 2001). Le rôle de la Convention du patrimoine mondial, quoiqu'étant déjà un mécanisme de conservation très appréciable puisqu'il couvre $8 \%$ du domaine mondial des aires protégées, pourrait être renforcé par une meilleure reconnaissance et un soutien plus appuyé de sa mise en œuvre, concentrés spécialement sur des mesures accrues pour les défis de conservation auxquels font face les sites de la Liste de l’UNESCO (UICN, 2009b).

En 2010, les Parties à la CDB (CDB, 2004b) passeront en revue le programme de travail adopté en 2004 pour les aires protégées et devraient veiller à garantir une meilleure synergie entre les nombreux instruments concernant les aires protégées. Les Parties à la CDB adopteront aussi un nouveau Plan stratégique et, peut-être, un cadre post-2010 pour la conservation de la biodiversité où les rôles critiques des aires protégées devraient être intégrés.

Comme le soulignait le commentaire très direct du représentant de la jeunesse au $V^{e}$ Congrès 
mondial des parcs, l'avenir des aires protégées est dans les mains des jeunes d'aujourd'hui. La CMAP travaille dur pour engager des jeunes dans les aires protégées, et les actions entreprises incluent la création, conjointement avec l'International Ranger Federation, du Young Conservationist Award, attribué chaque année à un jeune pour son travail exceptionnel pour les aires protégées ou la conservation, et qui encourage la participation des jeunes dans la Commission, avec comme objectif d'avoir 30\% de jeunes de moins de 35 ans d'ici 2012.

En conclusion, la conservation de la biodiversité par les aires protégées peut être une contribution importante à la construction d'une relation juste, équitable et durable entre les hommes et le reste de la nature. Pourtant cet objectif rencontre certains défis. Pour perpétuer la solide tradition de la contribution des aires protégées à la conservation, l'UICN doit continuer à soutenir les efforts visant à garantir qu'un système totalement représentatif des aires protégées est en place, avec une gestion efficace de ces aires et un financement correct pour assurer cette gestion. De plus, les opportunités fournies par les aires protégées comme mécanismes visant à soutenir la réduction de la pauvreté (Scherl et al., 2004), de mitigation et d'adaptation aux changements climatiques devraient être encouragées et réalisées. 


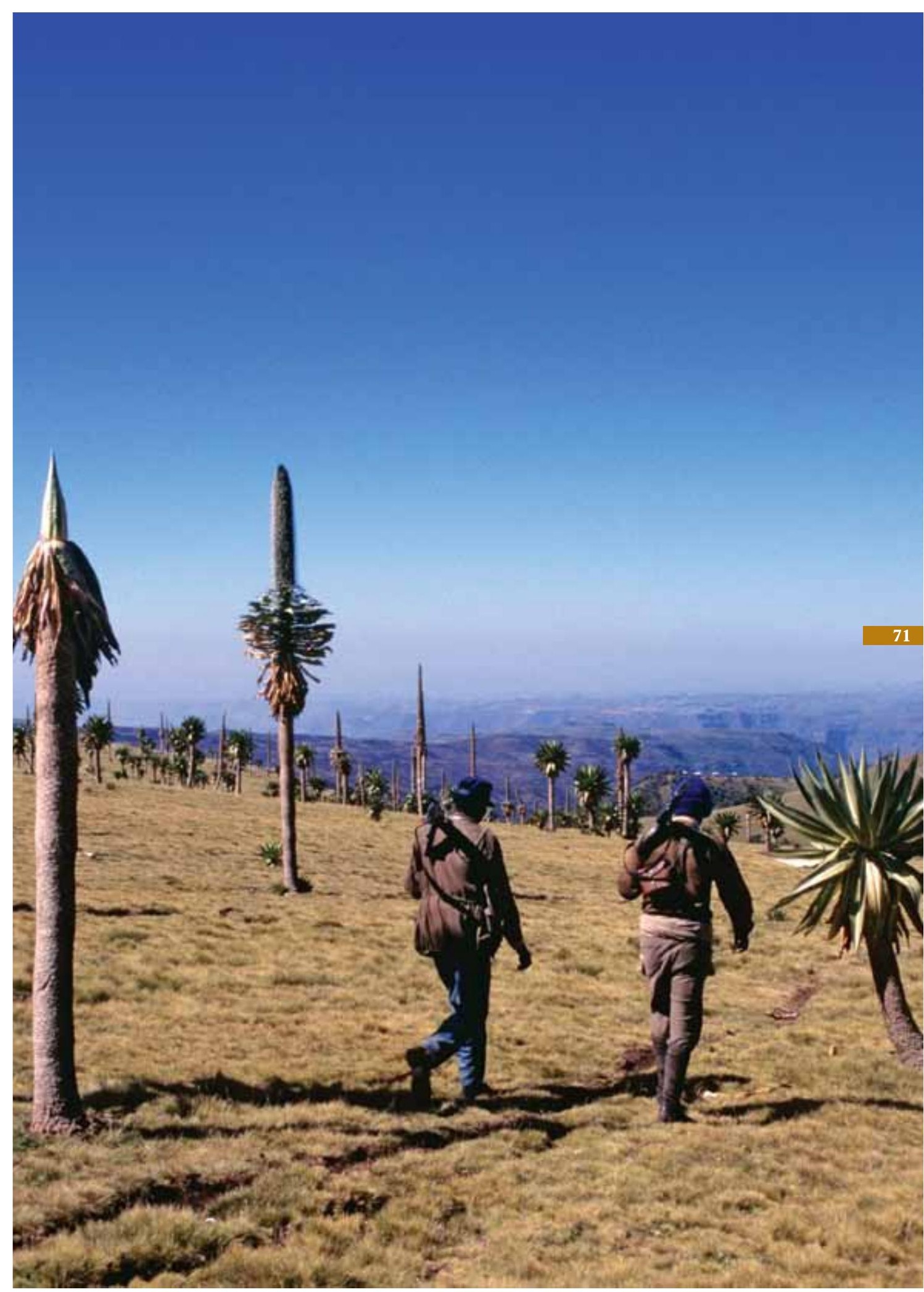




\section{Conservation des espèces : Défis d'aujourd'hui}

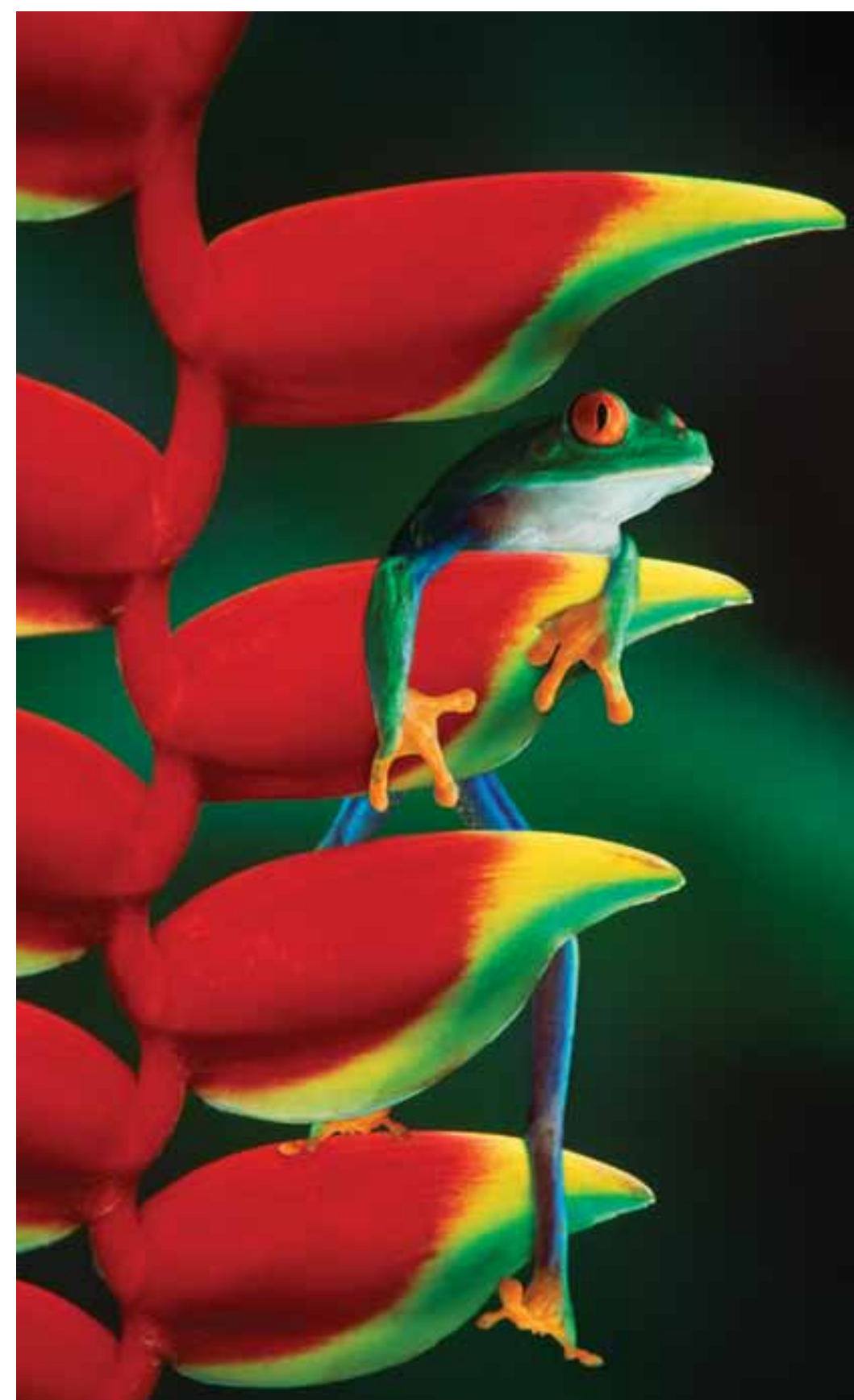


Le premier classement de la vie sur terre, initié par Linné et ses contemporains au milieu du $18^{\mathrm{ème}}$ siècle, fut fait au niveau de l'espèce. Depuis lors, au moins 1,7 millions d'espèces ont été formellement décrites et l'on estime que les espèces non encore découvertes sur terre sont entre 10 et 100 millions. Elément constitutif de la nature le mieux compris et le plus largement décrit, l'espèce est un centre d'intérêt naturel pour les actions de conservation. La première commission de l'UICN - à savoir le Service pour la survie des espèces qui fut créé en 1949, un an seulement après la création de I'UICN elle-même - était focalisée sur les espèces. Certains des programmes de conservation les mieux connus étaient fondés sur les espèces, comme le « Projet Tigre », lancé en 1973 en Inde suite à un recensement des tigres qui avait révélé l'effondrement du nombre des grands félins.

Depuis ces premiers jours de la conservation, les approches de la conservation de la nature se sont élargies pour inclure des approches à l'échelle des paysages et fondées sur les services écosystémiques. Mais la conservation basée sur les espèces reste une pierre angulaire de la conservation d'aujourd'hui, car elle offre une base mesurable qui peut facilement être entendue et comprise par le grand public. Les espèces phares, les espèces emblématiques, les hauts-lieux de la conservation et la plupart des indicateurs de biodiversité sont basés sur des mesures d'espèces. Au Forum mondial de nature, les espèces furent un sujet fréquent dans les présentations en atelier, et ce sont les problèmes des espèces qui font l'objet du plus grand nombre de Résolutions et de Recommandations du Congrès mondial de la nature $(\mathrm{CMN})$.
Pourtant, de nombreux problèmes subsistent pour la conservation basée sur les espèces, comme une compréhension encore rudimentaire de la diversité et du statut d'espèces appartenant à de nombreux taxons, spécialement les Invertébrés et les espèces marines. Citons aussi un débat toujours en cours concernant l'utilisation durable des espèces, les conflits entre hommes et faune sauvage et, paradoxalement, le besoin de contrôler certaines espèces (du genre invasif) pour que d'autres puissent survivre.

COMBLER LES LACUNES DES CONNAISSANCES SUR CERTAINES ESPÈCES

Les scientifiques n'ont peut-être identifié que 10\% des espèces existantes. Combler les lacunes entre ce que nous savons et ce que nous ne savons pas de 
la biodiversité est une tâche énorme que viennent compliquer de nombreux facteurs. Les technologies émergentes en matière de génomique " facilitent » l'identification des espèces grâce à des techniques comme les réactions en chaîne par polymérase, tandis que des avancées exponentielles dans la gestion de l'information permettent d'accéder plus rapidement aux sources de références (McNeely, 2002).

Néanmoins, il reste de sérieux défis, comme les moyens de s'accorder sur la définition d'une espèce, le renforcement des capacités en taxonomie et l'encouragement d'un accès ouvert à toutes les connaissances relatives aux espèces.

De nombreuses initiatives en matière d'informations sur les espèces sont en développement ou à divers stades de mise en œuvre : cela va du Système mondial d'information sur la biodiversité, une initiative intergouvernementale (http://www.gbif. org/), à l'Inventaire tous terrains, tous taxons (http:// www.dlia.org/atbi/). La plupart de ces initiatives dressent un catalogue des collections existant dans les musées, les herbariums et les académies. Certaines, comme le Service d'information sur les espèces de l'UICN (SIS), tentent aussi d'identifier et d'évaluer le statut actuel des populations in situ. Pourtant, l'ampleur de la tâche est énorme et il faudra un effort mondial coordonné pour permettre de bien comprendre le statut et les tendances d'un éventail représentatif de biodiversité au niveau des espèces.

La taxonomie, la façon dont les scientifiques cataloguent les espèces, est soumise à certaines contraintes qui freinent notre capacité de gérer les connaissances sur les espèces. Parmi les espèces décrites, une grande part l'ont été plus d'une fois, ce qui entraîne des problèmes de synonymie taxonomique. Par exemple, on estime que près de 13.000 nouvelles espèces sont décrites chaque année, mais le rythme actuel de résolution des synonymies réduit ce chiffre à 10.000 environ, à cause d'un taux de synonymie de $20 \%$ des espèces nommées (May, 1999). La controverse au sujet du statut des tortues d'eau douce chinoises - espèce vraie ou hybride ?- en est un cas d'école (Dalton, 2003). Les espèces nommées ne sont pas accessibles dans un travail de référence ou un index unique, ce qui pose un problème pour ceux qui voudraient une vue d'ensemble. Finalement, ce sont les taxonomistes qui deviennent une espèce en danger. Il est absolument nécessaire d'encourager les étudiants en biologie à faire carrière dans la taxonomie, en commençant par rendre cette discipline intellectuellement plus enrichissante et stimulante.

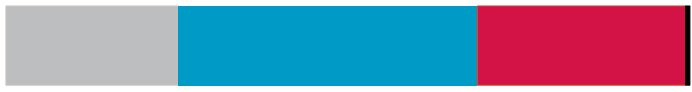

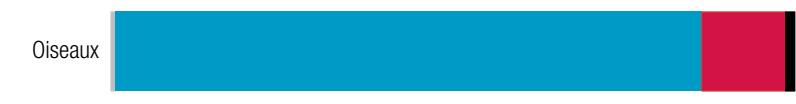

Mammifères

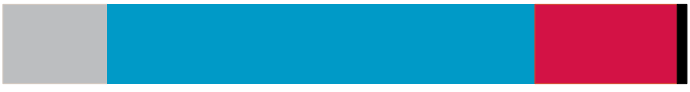

Gymnospermes
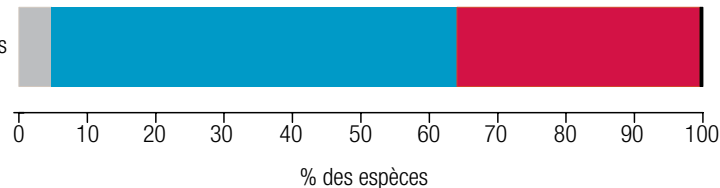

Éteint à l'état sauvage/Éteint

Vulnérable/En danger/En danger critique d'extinction

Préoccupation mineure/Quasi menacé

Données insuffisantes 
continue, encouragerait les gens à valoriser les ressources naturelles. Et lorsque des espèces sauvages et leurs habitats ont de la valeur, cela pousse à leur conservation et freine la conversion des habitats naturels en autres aménagements concurrents du territoire. La gestion d'animaux qui sont aussi du gibier, comme les cerfs ou les canards en Amérique du Nord, est souvent invoquée pour soutenir l'argument de l'utilisation durable comme tactique de conservation.

La valeur du commerce international de faune sauvage, forme d'utilisation économiquement mesurable, va croissant et elle est passée de 158,9 milliards de dollars au début des années 1990 à plus de 330 milliards en 2005 (Engler, 2008). La grande majorité (82\%) de cette valeur concerne les grumes et la pêche, mais le commerce est aussi considérable pour les produits forestiers non ligneux (27,7 milliards de dollars), les plantes d'ornement (13,4 milliards), les plantes médicinales (13 milliards) et les fourrures et produits dérivés (4,9 milliards).

En examinant les avis d'experts sur les facteurs sociaux et économiques du commerce de vie sauvage dans quatre pays du Sud-est Asiatique, on a identifié le revenu des consommateurs, le statut des lois et des réglementations, la disponibilité/ abondance des espèces en question et les prix comme facteurs incitant des changements du niveau du commerce d'espèces sauvages (TRAFFIC, 2008). Parmi les autres facteurs associés à ce commerce, on trouve de meilleures communications et une meilleure connectivité vers les marchés, de meilleures routes et infrastructures, et des coupes de bois illégales sur les sites concernés. La gestion du commerce de vie sauvage devra tenir compte de ces problèmes qui ne sont pas basés sur la conservation si l'on veut stopper la marée du commerce illégal et/ou non durable des ressources sauvages.

Le Groupe CSE/UICN de spécialistes de l'utilisation durable a identifié un ensemble de points auxquels il faudra faire attention pour atteindre une utilisation réellement durable, fondée sur l'expérience collective mondiale. Pour améliorer la probabilité que toute utilisation de ressource sauvage vivante soit durable, il faut tenir compte des principes suivants (UICN, 2000b) :

- Les réserves de produits biologiques et de services écologiques disponibles sont limitées par les caractéristiques biologiques intrinsèques des espèces et des écosystèmes, qu'il s'agisse de productivité, de résilience et de stabilité, qui sont elles-mêmes soumises à des changements environnementaux extrinsèques.

- Les structures institutionnelles de gestion et de contrôle exigent des incitants positifs et des sanctions négatives, une bonne gouvernance et une mise en œuvre à une échelle appropriée. De telles structures devraient inclure la participation des parties prenantes pertinentes et tenir compte des droits fonciers, des droits d'accès, des systèmes régulateurs, des savoirs traditionnels et des droits coutumiers.

- On attribue aux espèces sauvages vivantes de nombreuses valeurs culturelles, éthiques, écologiques et économiques qui peuvent constituer autant d'incitants pour leur conservation. Lorsque l'on peut attribuer une valeur économique à une espèce sauvage vivante, supprimer tout incitant pervers et internaliser les coûts et bénéfices, on peut créer des conditions favorables à un investissement dans la conservation et à l'utilisation durable de cette ressource. 
- Les niveaux et la fluctuation de la demande pour des ressources sauvages vivantes sont affectés par un éventail complexe de facteurs sociaux, démographiques et démocratiques, et ils vont probablement augmenter dans les années qui viennent. Il faut donc faire attention aussi bien à la demande qu'à l'offre pour promouvoir une utilisation durable.

Si le concept et les principes qui sous-tendent l'utilisation durable sont connus, la réalité veut que de nombreuses espèces soient utilisées impunément de façon non durable. Par exemple, le rapport de l'Organisation des Nations Unies pour l'alimentation et l'agriculture (FAO) sur l'état de la pêche dans le monde raconte l'histoire toujours très actuelle de la surexploitation, alors que les discussions de l'UE sur une politique commune en matière de pêche sont contrecarrées par des intérêts concurrentiels, avec pour résultat des « niveaux de capture réels qui sont 'inconnus' » (Economist, 2009). Brashares et al. (2004) rapportent aussi que, en Afrique de l'Ouest, les captures de poissons réduites à cause de la surexploitation aboutissent à une pression plus grande sur les autres ressources naturelles, et spécialement sur la viande de brousse.

Une utilisation non soutenable se passe aussi dans d'autres secteurs que la pêche. La consommation de viande d'animaux sauvages provoque des extinctions locales (Milner-Gulland et al., 2003) et est de plus en plus inquiétante, surtout maintenant que la sécurité alimentaire attire l'attention des décideurs. En plus des impacts directs sur les espèces consommées, les scientifiques notent des changements plus importants dans les écosystèmes suite à l'enlèvement de ces espèces, notamment des changements de la diversité végétale (Nuñez-Iturri et Howe, 2007).

Une gouvernance et une orientation efficaces sont nécessaires pour étayer l'utilisation durable des ressources. Par exemple, assurer la conservation de forêts tropicales productives est l'objectif des «Directives OIBT/UICN pour la conservation et l'utilisation durable de la biodiversité dans les forêts tropicales productrices de bois » récemment adoptées, qui devraient constituer le cadre pour l'utilisation durable des produits forestiers (ligneux et non ligneux, y compris la viande de brousse) en dehors des aires protégées (Décision OIBT 6 (XLIV) - (http://www.itto.int/fr/decisions ).

Néanmoins, certains mécanismes mis en place pour gérer l'utilisation des ressources ont leurs limites, dont la moindre n'est pas la pression politique pour fixer des quotas insupportablement élevés, comme la politique européenne en matière de pêche citée plus haut. Un autre exemple est, dans le cas de la Convention sur le commerce international des espèces de faune et de flore sauvages menacées d'extinction (CITES), le danger que, pendant la transition entre un type de réglementation et un autre, l'exploitation ne connaisse une hausse marquée (Rivalan et al., 2007).

Les nouvelles technologies évoluent, comme les empreintes « digitales » des cornes de rhinos ou les techniques moléculaires utilisées pour identifier les produits dérivés d'oiseaux, de poissons et de baleines sur les marchés (Palsbøll et al., 2006 ; Baker et al., 2007), mais Rubinoff (2006) prévient que les codes-barres $\mathrm{ADN}$ ne donnent que certaines des informations nécessaires à l'identification et que, spécialement pour les espèces moins connues, il faut en tenir compte dans les décisions concernant leur utilisation. L'analyse isotopique de l'eau (Bowen et al., 2005) est aussi un moyen possible de gérer le commerce de produits d'origine sauvage. Les isotopes stables de l'eau des précipitations et de la neige varient beaucoup et systématiquement sur une base géographique, et leur présence dans les tissus animaux suite à leur ingestion signifie que ce sont de possibles traceurs de l'origine géographique. Cependant, il faut faire un travail de calibration par rapport à des échantillons d'origine connue.

L'utilisation durable d'au moins certaines ressources naturelles fait partie intégrante de tout programme de développement durable mais cela reste un sujet hautement controversé dans la communauté 
de la conservation (Hutton et Leader-Williams, 2003). L'attention à tous ces facteurs, en plus des caractéristiques biologiques et écologiques de la ressource impliquée, est une des clés du succès, de même qu'une meilleure collaboration dans l'identification des approches les plus susceptibles de mener à une réduction de toute utilisation illégale et/ou non soutenable (TRAFFIC, 2008). Il faut notamment faire très attention en créant des incitants positifs pour la conservation et l'utilisation durable. La résolution complète des controverses entourant l'utilisation d'une espèce en particulier restera un défi car certains groupes pourraient ne jamais accepter aucune utilisation de cette espèce.

\section{CONFLITS HOMMES-FAUNE SAUVAGE}

Les grands prédateurs, comme les grands félins, les crocodiles et les loups, et d'autres espèces comme les éléphants représentent de réels dangers pour la vie humaine et pour les moyens de subsistance car ils détruisent des biens comme les récoltes ou le bétail. Par exemple, une étude des pertes de bétail chez des agriculteurs qui vivent dans le Parc National de Jigme Singye Wangchuk, au Bhoutan, a révélé des pertes qui pouvaient aller jusqu'aux deux-tiers du revenu annuel des ménages (Wang et Mc Donald, 2006). Les facteurs qui affectent le taux de prédation incluaient une surveillance relâchée, des pratiques de gardiennage inappropriées, le surpâturage, le manque d'étables correctes pour y rassembler le bétail pendant la nuit et la distance entre le foyer et le lieu de pâturage.

Ces conflits ne sont pas neufs ; dès les années 1700 , des rapports signalent des loups mangeurs d'hommes en Europe et des tigres mangeurs d'hommes en Asie. Mais comme les espaces vraiment sauvages rétrécissent et que les populations humaines s'agrandissent, la probabilité de confrontations et de conflits augmente. En plus des causes directes de conflits, il y a des impacts indirects comme la perte de revenus due au temps passé à régler ces problèmes avec les espèces.
Les réponses peuvent se diviser entre la protection, la mitigation (schémas de compensation et d'incitation) et la prévention (en changeant les pratiques de gestion et en relocalisant), et les approches réussies combinent des approches biologiques à court terme et des approches sociales à long terme (Distefano, 2005 ; Thouless, 2008). Il est nécessaire de résoudre d'abord le conflit et puis d'augmenter la tolérance avant de mettre en œuvre une planification de partage des profits et d'aménagement du territoire pour pouvoir empêcher l'occurrence de nouveaux conflits. Les étapes clés sont les suivantes :

- Une attention à la planification de l'aménagement du territoire. La fragmentation de l'habitat a souvent un lien direct avec les conflits. Dans certains cas, les zones tampons peuvent être efficaces et on peut les créer par échanges de terrains.

- Une responsabilisation communautaire. Un sentiment d'appropriation de la faune sauvage aboutit souvent à la gestion de la faune par les populations locales.

- Une création d'incitants économiques pour renforcer la tolérance.

Lorsque des conflits éclatent à travers des paysages entiers, il est difficile de résoudre les problèmes au cas par cas, et il est important de développer un cadre de réponses impliquant la collaboration de toutes les parties prenantes. Bien que l'on ait mis au point des méthodes standardisées pour mesurer les conflits avec des éléphants, il est urgent de faire la même chose pour d'autres espèces, pour disposer de moyens fiables pour comparer l'intensité des conflits entre les sites afin de prioriser les réponses.

\section{LES ESPÈCES ENVAHISSANTES}

Les espèces exotiques envahissantes constituent une grave menace pour la biodiversité et pour les services écosystémiques; elles comptent parmi les cinq menaces principales citées par l'Évaluation des écosystèmes pour le millénaire 
(EM) (2005) pour l'intégrité des écosystèmes. Les espèces envahissantes peuvent causer la perte de biodiversité, des changements de la composition chimique de l'eau, des modifications des processus biochimiques, des changements hydrologiques et des perturbations des cycles alimentaires (Dukes et Mooney, 2004 ; Ehrenfeld, 2003) ; elles peuvent aussi changer la disponibilité de lumière, d'air, de nourriture, d'abri et de sites de reproduction. Pour les oiseaux, Butchart $e t$ al. (2008) notent que des espèces envahissantes menacent les oiseaux de plusieurs façons, notamment la prédation des adultes, le stress sur la reproduction par la prédation des œufs et des poussins, et la dégradation des habitats (particulièrement par des herbivores ou des plantes invasifs). Un tiers des espèces d'oiseaux en danger dans le monde sont menacées par des espèces envahissantes, surtout à cause de la prédation par les carnivores et les rongeurs.

Baillie et al. (2004) ont noté que les espèces envahissantes sont un risque majeur pour $11 \%$ des espèces menacées d'amphibiens et pour $8 \%$ des espèces menacées de mammiferes pour lesquelles des données sont disponibles. Ils rapportaient aussi que les espèces insulaires étaient particulièrement vulnérables et indiquaient que $67 \%$ des oiseaux menacés dans des îles océaniques étaient affectés par des espèces envahissantes, à comparer avec $8 \%$ des oiseaux continentaux. Darwall et al. (2008) rapportent que $85 \%$ des poissons menacés en Afrique australe, $55 \%$ des poissons d'eau douce menacés d'Europe et un peu moins de $45 \%$ des poissons d'eau douce menacés à Madagascar sont affectés par des espèces envahissantes. Dans ce dernier cas, c'est en grande partie le résultat de la mise en œuvre d'un plan destiné à rétablir les pêcheries locales en introduisant 24 espèces de poissons non indigènes (Benstead et al., 2003).

Les caractéristiques qui définissent une espèce envahissante potentielle comprennent des facteurs intrinsèques propres aux espèces envahissantes et aux habitats qui vont être envahis. Howard et Ziller (2008) en donnent une liste :

- Taux de croissance rapide ;

- Capacité de grandir correctement dans des conditions arides ou difficiles en général (large tolérance environnementale) ;

- Production de nombreux fruits ou semences bien protégés ;

- Production de fruits et de graines (ou autres propagules) à des phases précoces $\mathrm{du}$ développement et de la croissance ;

- Capacité d'être dispersé largement par le vent ou l'eau, ou par des animaux qui s'en nourrissent ou transportent leurs propagules; et

- Compétition efficace avec d'autres plantes.

Le Groupe CSE/UICN de spécialistes des espèces envahissantes (GSEE) offre un réseau d'expertise sur les espèces envahissantes et une base de données sur les espèces envahissantes les plus menaçantes (www.issg.org). Le Programme mondial sur les espèces envahissantes (GISP www.gisp.org) est un partenariat international (incluant l'UICN) qui lutte contre la menace des espèces envahissantes en augmentant la connaissance et la sensibilisation à cette problématique et en développant les outils et les méthodes nécessaires pour empêcher et contrôler les invasions.

Il peut être difficile de prédire le potentiel invasif d'une espèce particulière parce que les invasions, comme toute entreprise humaine, peuvent être déjouées par des problèmes de timing et de changements (Baskin, 2002). Bien que les invasions biologiques soient des problèmes écologiques, évolutifs et socioéconomiques complexes, on commence à bien les comprendre, et spécialement l'écologie des caractères invasifs et de la vulnérabilité de l'habitat face aux invasions. 
Ces connaissances sont essentielles pour déterminer combien d'efforts il faut investir dans le contrôle des espèces envahissantes qui se sont déjà établies ou pour clarifier les compromis que les gestionnaires et les planificateurs fonciers vont devoir envisager.

Léradication et le contrôle des espèces envahissantes ont utilisé différentes tactiques et stratégies (Wittenburg et Cock, 2001 ; Veitch et Clout, 2002). La lutte chimique contre des espèces végétales envahissantes, parfois combinée avec l'arrachage mécanique, la coupe ou l'élagage, s'est avérée très utile pour contrôler au moins certaines plantes envahissantes, mais elle n'a pas été particulièrement convaincante pour en éradiquer. On a aussi tenté le contrôle biologique de certaines espèces envahissantes. L'idée, derrière cette approche, c'est de profiter de relations écologiques comme la compétition, la prédation, le parasitisme ou l'herbivorie qui existent entre un envahisseur et un autre organisme non indigène introduit comme agent de contrôle (lequel est souvent du même habitat naturel d'origine que l'espèce envahissante). Les résultats sont mitigés. Par exemple, le papillon parasite du figuier de Barbarie (Cactoblastis cactorum) utilisé pour lutter contre des espèces envahissantes d'Opuntia en Australie, a envahi récemment les États-Unis et menace sérieusement l'espèce d'Opuntia native de la région (Stiling, 2002).

Les cas d'éradication qui ont réussi ont en commun trois facteurs clés : des caractéristiques biologiques particulières des espèces (par exemple, une faible capacité de se disperser), des ressources économiques suffisantes pour une période assez longue, et le soutien général des organismes concernés et du public (Mack et al., 2000). Lorsque l'éradication complète n'est pas possible, ou pas souhaitée, comme dans le cas d'espèces natives qui deviennent envahissantes parce que leur aire de répartition s'agrandit, on peut tenter certaines mesures de " contrôle de routine " visant à maintenir les populations de l'espèce envahissante sous un seuil acceptable. Mais les contrôles chimiques et mécaniques posent de nombreux problèmes, notamment leur coût élevé et la réticence du public pour certaines pratiques (Mack et al., 2000).

\section{L'AVENIR DE LA CONSERVATION DES ESPÈCES}

Les paradigmes des services écosystémiques, la conservation en faveur des pauvres et les approches de la conservation fondées sur les droits, tiennent le devant de la scène, mais ces approches requièrent toutes une attention continue pour le rôle fondamental que les espèces jouent et qui est à la base de ces paradigmes. Dans les avancées permanentes de la conservation, les approches par espèces restent au centre des démarches. Nous devons continuer à employer tous les instruments de la boîte à outils de la conservation des espèces, depuis le développement et la mise en œuvre des plans d'action pour les espèces à la réintroduction, la gestion ex situ et plus encore.

Dans la prochaine décennie, on ne devrait pas permettre qu'une seule espèce disparaisse sciemment. La communauté de la conservation devrait continuer à participer au suivi et à l'évaluation du statut et des menaces sur les espèces, et notamment aider au développement d'indicateurs et aux comptes-rendus réguliers. Il sera vital de travailler en vue d'une meilleure compréhension des paramètres qui définissent ce qu'est une « utilisation durable » des espèces et d'encourager les gestionnaires de ces espèces à faire usage de ces connaissances. De même, le monde de la conservation devrait promouvoir tous les efforts possibles pour gérer et contrôler les espèces envahissantes. 


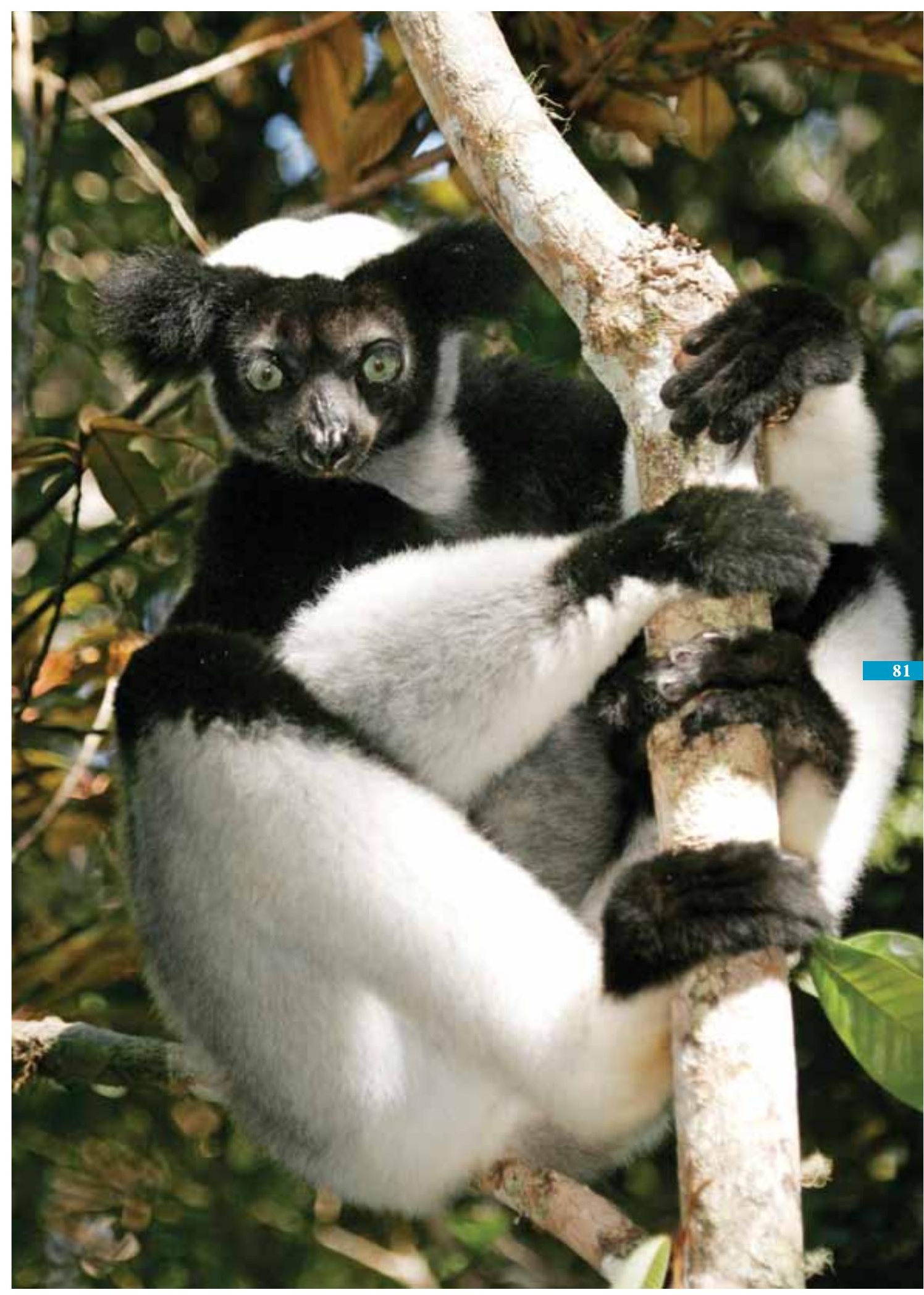


8. Un avenir après le pétrole : qu'est-ce

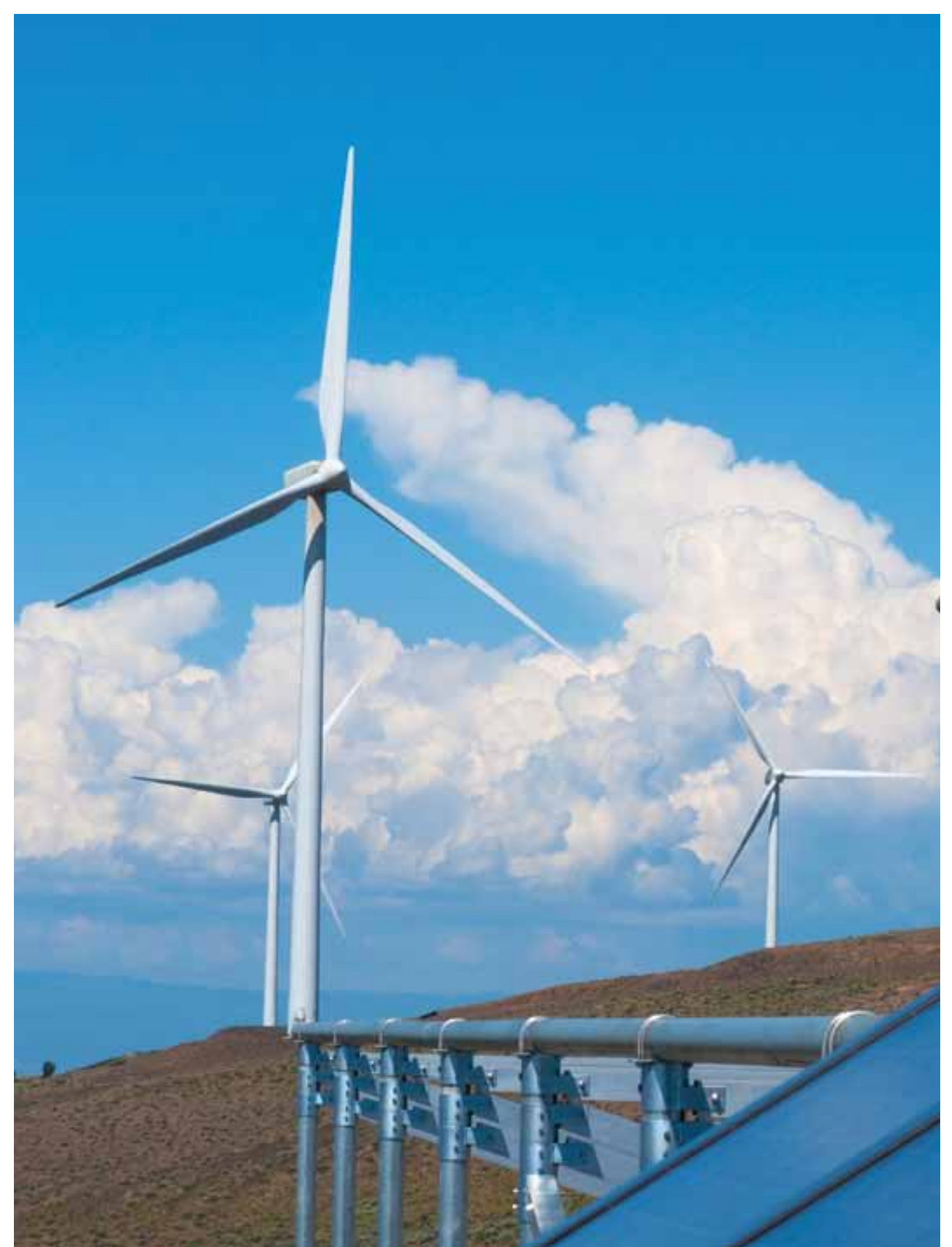


L'utilisation de l'énergie donne aux hommes un avantage considérable sur toutes les autres espèces. Depuis que nos ancêtres ont pu transformer le carbone en énergie en utilisant le feu, l'utilisation de l'énergie a toujours tenu une place extrêmement importante dans nos relations avec le reste de l'environnement. Le feu nous a gardés au chaud en hiver, nous a permis de cuire la nourriture, de dégager les sous-bois pour faciliter la chasse et de devenir plus actifs pendant la nuit. Puis les hommes ont maitrisé l'eau pour faire fonctionner les moulins, et le vent pour propulser les bateaux et faire tourner les moulins à vent, pour actionner des pompes à eau et moudre le grain, et ainsi de suite. Cela leur a permis de convertir d'autres formes d'énergie et d'étendre alors leurs niches écologiques et aussi leurs populations. Le charbon a alimenté la révolution industrielle et reste la source d'énergie principale dans de nombreux pays (les ÉtatsUnis, la Chine et l'Inde pour n'en citer que quelques-uns). L'utilisation généralisée du pétrole au $20^{\mathrm{ème}}$ siècle a suscité beaucoup de nouvelles utilisations de l'énergie, comme la grande mobilité que permettent les automobiles et les avions.

Lorsque le prix du pétrole a atteint 147 US\$ le baril durant l'été 2008, de nombreux conservationnistes furent écartelés entre jubilation et désespoir. Côté jubilation, ce prix extrême indiquait clairement combien nos sociétés modernes étaient devenues dépendantes du pétrole et il soulignait le besoin de commencer à penser sérieusement à un avenir énergétique alternatif. Beaucoup pensaient que cette poussée extrême était un symptôme du " pic pétrolier mondial ", le moment où la moitié du pétrole disponible avait déjà été exploitée, signifiant ainsi que la fourniture de pétrole allait décliner à partir de ce jour (Deffeyes, 2005). Si la demande de pétrole reste forte, les prix doivent rester élevés, ce qui devrait susciter des investissements dans des sources d'énergie alternatives qui sont beaucoup moins nuisibles pour le climat. Dans ce cas, la demande de pétrole a chuté, mais la poussée des prix a servi d'avertissement : il faut chercher des alternatives.

Il faut replacer l'inquiétude au sujet des prix du pétrole dans le contexte des projections de demandes d'énergie. Le World Energy Outlook 2008 prédit une croissance de $50 \%$ de la demande en énergie d'ici 2030 , et $70 \%$ de cette demande 
accrue devraient venir de pays en développement, $30 \%$ pour la seule Chine (OCDE/AIE, 2008).

Les combustibles fossiles devraient encore constituer la majorité de toutes les énergies pour les prochaines décennies, mais maintenant que le pétrole montre des signes d'épuisement, il est temps d'envisager d'autres options énergétiques. Ces réflexions sont suscitées par des inquiétudes au sujet des changements climatiques, de la sécurité énergétique et de la distribution équitable des bénéfices de l'énergie.

Tous les choix énergétiques possibles ont des impacts potentiels sur la biodiversité. Par exemple, aux combustibles fossiles on associe surtout une contribution aux changements climatiques et à la pollution de l'air, avec des impacts conséquents sur la nature. Pourtant, nous devons aussi faire attention aux impacts directs des marées noires sur les écosystèmes aquatiques et marins, et aux impacts indirects dus au développement des puits de pétrole, des infrastructures et des activités humaines qui y sont associées dans certaines régions reculées (comme l'Arctic National Wildlife Refuge, en Alaska) très importantes pour la conservation de la biodiversité.

\section{OPTIONS POUR UN AVENIR APRÈS-PÉTROLE}

\section{Efficacité énergétique}

De loin la plus rapide et la moins chère, la seule option qui n'a pas d'impact négatif sur l'environnement est la conservation de l'énergie - utiliser moins d'énergie, simplement en réduisant sa consommation ou en rendant les processus de production plus efficaces. Par unité d'extrant économique, le Japon, par exemple, utilise seulement $10 \%$ de ce qu'utilise la Chine. Les prix élevés du pétrole indiquent clairement que la conservation est réalisable, que ce soit en empruntant les transports en commun, en utilisant des appareils électriques plus efficaces ou en isolant mieux les bâtiments. Les particuliers peuvent aussi faire de sérieuses économies en la matière. L'efficacité et la conservation de l'énergie doivent rester la première réponse lorsqu'on aborde un avenir après-pétrole ; elles présentent de multiples avantages pour tout, des émissions de carbone à la biodiversité en passant par la sécurité énergétique.

\section{Sources d'énergie alternatives}

\section{Nucléaire}

Même si l'énergie nucléaire n'a plus la cote depuis la dernière partie du siècle dernier, le nucléaire revient sur la table des négociations maintenant que le pétrole est cher et que le climat change. Les partisans arguent que le nucléaire est très propre en ce qui touche son impact sur le climat, qu'il a prouvé son efficacité dans les pays qui recourent en grande partie à l'énergie nucléaire (comme la France et la Suisse) et qu'il peut encore être beaucoup amélioré grâce aux nouvelles technologies. Pourtant, " propre » ne signifie pas forcément "vert ». Les opposants brandissent les arguments éternels de l'élimination des déchets et du risque de prolifération et de ses conséquences sur la sécurité mondiale, des investissements coûteux, des dangers inhérents de fusion, de la forte demande en eau pour le refroidissement et de la réalité incontestable du fait que le principal combustible, l'uranium, est une ressource non renouvelable (avec ses impacts miniers) peu abondante. De nouveaux types de réacteurs, comme les surgénérateurs et les réacteurs à lit de boulets, peuvent être une réponse partielle à ce type de préoccupation, mais ils n'ont pas encore prouvé leur viabilité technique et toute utilisation commerciale est encore loin dans le futur. De plus, le coût réel de l'énergie nucléaire est très difficile à déterminer parce que les frais de développement sont rarement pris en compte, pas plus que le coût du décommissionnement 
des réacteurs et de l'élimination des déchets nucléaires. De plus, l'énergie nucléaire semble exiger le support des plus hauts niveaux gouvernementaux. Par exemple, aux États-Unis, l'électricité nucléaire peut bénéficier de 32 types de subsides différents et c'est une des façons les plus inefficaces d'atténuer les émissions de gaz à effet de serre (GES) (Earthtrack, 2008).

\section{Biomasse}

La biomasse est une forme d'énergie ancienne. Actuellement, plus de 2,5 milliards de personnes dans le monde dépendent de formes traditionnelles de biomasse, comme le bois, le charbon de bois et les excréments d'animaux pour la lumière, le chauffage et la cuisson des aliments (OCDE/AIE, 2008), et cela peut représenter jusqu'à $90 \%$ de la demande primaire en énergie des ménages dans de nombreux pays en développement. L'utilisation de biomasse traditionnelle pour l'énergie n'est pas nécessairement non soutenable en soi, mais le rythme et la méthode d'utilisation peuvent causer des problèmes environnementaux et sanitaires. La première euphorie suscitée par la production industrielle de biocarburants est retombée lorsqu'on a réalisé que les terres utilisées pour la production de biocarburants risquaient d'être détournées d'autres utilisations importantes, comme la production de nourriture. Les cultures destinées aux biocarburants sont typiquement des monocultures, une stratégie risquée dans la mesure où parasites et maladies ont tendance à se disperser plus rapidement dans des monocultures que dans des polycultures. L'UICN et le Programme mondial sur les espèces envahissantes ont averti des risques d'espèces envahissantes dans les plantations de biocarburants. De plus, les avantages des biocarburants se révèlent éphémères, et beaucoup font en réalité plus de tort que de bien, selon la façon et l'endroit où le combustible est produit (Howard et Bringenzu, 2009).

Les impacts des biocarburants sur la biodiversité peuvent être importants. Il est intéressant de noter que les cultures de biocarburants partagent de nombreuses caractéristiques avec les espèces envahissantes : une croissance rapide, une grande productivité, l'adaptabilité à une gamme variée de sols et de conditions climatiques et la résistance aux parasites et aux maladies. Le palmier nipa, par exemple, a envahi et colonisé plus de $200 \mathrm{~km}^{2}$ sur la côte Atlantique du Nigeria et peut produire un biocarburant beaucoup plus intéressant à l'hectare que la canne à sucre, selon certains experts. Il faudrait donc traiter toutes les cultures introduites pour faire des biocarburants comme des espèces potentiellement envahissantes jusqu'à preuve du contraire. Récolter simplement des espèces envahissantes à problèmes comme la jacinthe d'eau, les Lantana camara et le palmier nipa pourrait constituer une option intéressante de matière première des biocarburants, mais cela ne les contrôlera pas pour autant et pourrait avoir un effet pervers si un marché se crée pour ces espèces envahissantes et encourage leur dispersion et de nouveaux dommages pour la biodiversité.

Dans la Résolution 4.082, l'UICN en appelle aux gouvernements qui ont choisi de développer une bioénergie à grande échelle, voire industrielle, pour qu'ils définissent et appliquent des critères pour une production et une utilisation de biomasse écologiquement durables, socialement 
adaptées et économiquement viables, qui :

a. N'entraînent aucune perte nette de biodiversité ;

b. Ne causent aucune émission due à la déforestation et à la dégradation des forêts et d'autres écosystèmes naturels ;

c. N'affectent pas négativement la sécurité alimentaire ;

d. Assurent que l'énergie de la biomasse réduit les émissions nettes de gaz à effet de serre par rapport aux alternatives ;

e. Rapportent des bénéfices pour les producteurs de matières premières combustibles, particulièrement aux groupes vulnérables que sont les pauvres, les femmes et les populations autochtones des régions rurales ;

f. Exigent des méthodes de production qui utilisent l'eau de façon efficace et durable, favorisent la plantation d'espèces natives et évitent de planter des espèces potentiellement envahissantes ; et

g. Découragent le commerce de bioénergie produite de façon non durable, en utilisant des mesures non protectionnistes.

La Table ronde sur les biocarburants durables a développé 12 principes qui encadrent l'orientation pour un développement plus durable des biocarburants dans le futur (RSB, 2008), et le Conseil International sur la gouvernance des risques a fourni des lignes directrices sur la façon de gérer les risques que posent les biocarburants (IRGC, 2008a).

\section{Hydroélectricité}

L'hydroélectricité fournit 2\% de la demande d'énergie primaire mondiale et c'est la plus importante source d'énergie produite de façon durable (World Energy Outlook, 2008). La plus grande partie du potentiel hydroélectrique est totalement exploitée dans les pays développés, les autres systèmes hydrologiques étant souvent protégés. Mais l'on s'attend à une forte croissance dans les pays en développement. Certains pays, comme le Népal, la RPD Lao, et le Congo, pourraient être les « batteries » de leurs régions respectives en raison des montagnes escarpées et des grands systèmes fluviaux. Pourtant, de nombreux barrages hydrauliques sont férocement décriés parce qu'ils limitent les flux d'eau dans les bassins de rivière et que cela a des retombées sur les moyens de subsistance comme la pêche, et aussi à cause du déplacement forcé de la biodiversité et des communautés pour la création des réservoirs.

Pour trouver un moyen de contrebalancer les risques sociaux et environnementaux liés à la création d'énergie renouvelable, l'UICN s'engage dans le Forum d'évaluation de la durabilité hydroélectrique (Hydropower Sustainability) Assessment Forum - HSAF) qui veut créer un outil d'évaluation de la durabilité largement reconnu, pour mesurer et orienter les performances dans le secteur hydroélectrique, en s'appuyant sur la Commission mondiale des barrages (que l'UICN a aidé à créer). L'UICN attire l'attention sur des points particuliers afin d'encourager le secteur hydroélectrique à gérer de façon durable les sources des bassins versants et d'assurer des débits d'eau vers l'aval suffisants pour préserver les écosystèmes situés plus bas et les services qu'ils fournissent aux gens.

L'UICN travaille dans le monde entier sur des projets qui montrent qu'il est important de préserver le débit de tous les cours d'eau, y compris ceux qui ont des barrages. Au Vietnam, par exemple, une évaluation du cours du Huong a montré clairement comment les modifications du débit de la rivière avaient affecté les revenus économiques et la santé de l'écosystème. Les autorités du bassin ont pu déterminer quelles options respectaient les objectifs économiques tout en protégeant les écosystèmes de l'aval et leurs services. L'application du concept de flux environnemental permet de prendre des décisions intégrées au sujet d'une utilisation de l'eau qui reste dans les limites disponibles, pour respecter les priorités en matière de croissance 
économique, de moyens de subsistance et de conservation durables, et d'augmenter ainsi la durabilité des infrastructures hydrologiques, dont l’hydroélectricité.

\section{Éolien}

D'après le Conseil mondial de l'énergie éolienne (GWEC), en 2009 l'ensemble des installations éoliennes dans le monde totalise quelque 120.798 mégawatts (MW). Cette capacité a augmenté de 25\% par an ces dernières années. Les ÉtatsUnis ont récemment dépassé l'Allemagne, avec une capacité totale de $25.170 \mathrm{MW}$, ce qui équivaut à plus d'un cinquième de la capacité mondiale.

L'Allemagne peut produire $23.903 \mathrm{MW}$

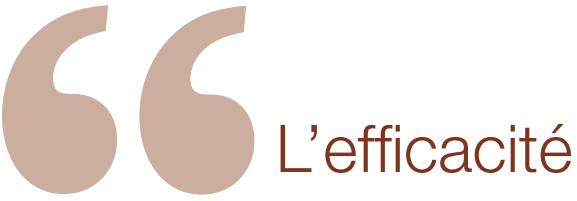
et l'Espagne 16.754 MW. La Chine aussi augmente très vite sa capacité, avec 12.210 MW, dépassant l'Inde et ses $9045 \mathrm{MW}$.

Un facteur critique pour la réussite du développement de l'énergie éolienne est le soutien gouvernemental, qui implique souvent des tarifs préférentiels, des subsides ou des réductions de taxes et la conservation de l'énergie doivent rester la première réponse lorsqu'on aborde un avenir après-pétrole. visant à encourager des formes d'énergie plus propres.

Les oiseaux et les chauves-souris sont victimes des parcs à éoliennes, souvent en heurtant les hélices. Chez les oiseaux, les passereaux qui migrent pendant la nuit sont les plus vulnérables et, chez les chauves-souris, ce sont les espèces migratrices qui se perchent dans les arbres (NRC, 2007).
Les raisons de la forte mortalité des chauvessouris vont des espèces qui se perchent dans les arbres et qui sont attirées par les hauts pylônes des parcs d'éoliennes à l'augmentation possible de la quantité d'insectes due aux changements d'utilisation des terres liés à la construction de parcs à éoliennes, ou encore à l'attraction que constituent les sons créés par les turbines et à la dépression causée dans leurs poumons par le changement abrupt de la pression de l'air (Kunz et al., 2007b). Pour gérer les impacts que les parcs à éoliennes pourraient avoir sur les oiseaux nocturnes et sur les chauves-souris, Kunz et al. (2007a) ont publié des lignes directrices à consulter lors de la construction et du fonctionnement de ces sites.

Du côté positif, les terres occupées par des parcs à éoliennes peuvent toujours servir de cultures ou de réserves de la biodiversité, en fonction des souhaits des communautés concernées. De même, les partisans des parcs éoliens marins soutiennent qu'ils sont avantageux pour la pêche parce qu'ils constituent une " aire protégée " pour la reproduction des poissons. Pourtant, certaines études indiquaient que les vibrations causées par les turbines pouvaient perturber au moins certaines espèces de poissons et de mammiferes marins. Il reste donc à prouver que les parcs à éoliennes marins seront en pratique positifs pour la pêche. 


\section{Solaire}

Même si elle ne satisfait aujourd'hui que $0,1 \%$ de la consommation d'énergie mondiale, l'énergie solaire photovoltaïque a un potentiel énorme, spécialement dans les pays très ensoleillés. Le secteur de l'énergie solaire se développe rapidement pour produire de l'électricité ; cela va des nouvelles avancées des petits éléments photovoltaïques intégrés dans des immeubles aux grandes tours solaires thermiques. Si l'aménagement du territoire et l'accès des communautés locales aux derniers développements solaires sont encore préoccupants, le principal obstacle à l'utilisation plus généralisée de l'énergie solaire est le coût élevé des investissements nécessaires. De plus, les matériaux semi-conducteurs utilisés pour construire les cellules solaires de nouvelle génération sont des minerais extraits comme le gallium et l'indium, tous deux extrêmement rares, et cela signifie que, pour être les plus utiles possibles, les développements de l'énergie solaire devraient se concentrer sur les pays où le soleil est le plus généreux. Des installations solaires placées en hauteur peuvent favoriser dans leur ombre la croissance d'herbes qui seront un habitat pour quelques espèces au moins.

\section{Géothermie}

L'Islande est la championne de la géothermie où elle puise $26 \%$ de sa demande totale en électricité, mais d'autres pays ont aussi un potentiel géothermique. La Nouvelle-Zélande, l'Indonésie, le Japon et la Russie ont un potentiel certain. Le Massachusetts Institute of Technology (MIT) (2007) rapporte qu'avec un investissement raisonnable dans la recherche et le développement, l'énergie géothermique pourrait fournir aux États-Unis 100 gigawatts (GW) dans les 50 prochaines années. Dans une perspective de développement, il est intéressant de noter que le Grand Rift d'Afrique de l'Est a un potentiel géothermique de 14.000 MW alors que 200 MW seulement sont captés par le Kenya qui est en tête dans la région, ce qui représente $14 \%$ de son électricité (Economist, 2008). Les impacts environnementaux sont négligeables.

\section{Vagues et marées}

L'océan possède une quantité phénoménale d'énergie avec la force de ses vagues et de ses marées. On étudie de nombreuses façons de capter cette énergie. Le Royaume-Uni est l'investisseur le plus en pointe, avec une politique fermement en faveur de l'énergie océanique. Le barrage marémoteur de 10 miles proposé pour l'estuaire de la Severn, dans le sud-ouest de l'Angleterre, exploiterait le deuxième différentiel de marées du monde et pourrait produire 5\% des besoins du Royaume-Uni en électricité, équivalant à huit centrales électriques classiques fonctionnant au charbon. Mais il va aussi toucher les zones humides et les réserves d'oiseaux locales. Cet exemple montre bien que les écosystèmes côtiers subissent déjà des demandes nombreuses et parfois conflictuelles et que, par conséquent, ils comptent parmi les écosystèmes les plus dégradés.

\section{ÉNERGIE ET DÉVELOPPEMENT DURABLE}

Les sociétés ont besoin d'énergie pour vivre et prospérer, mais l'accès à une énergie accessible et durable est encore refusé à de nombreuses régions du monde. Elsayed (2009) rapporte que dans plus de 30 pays, presque tous d'Afrique subsaharienne, moins de la moitié de la population a accès à l'électricité (Figure 8.1). Une carte des pays ayant recours à des combustibles solides (combustibles traditionnels comme le bois, les excréments, les résidus agricoles et le charbon) est presque une image en miroir de la Figure 8.1, qui montre une lourde dépendance en Afrique subsaharienne et dans les pays en développement d'Asie (Elsayed, 2009).

Le recours aux énergies traditionnelles est un risque particulier pour les femmes et les enfants. 


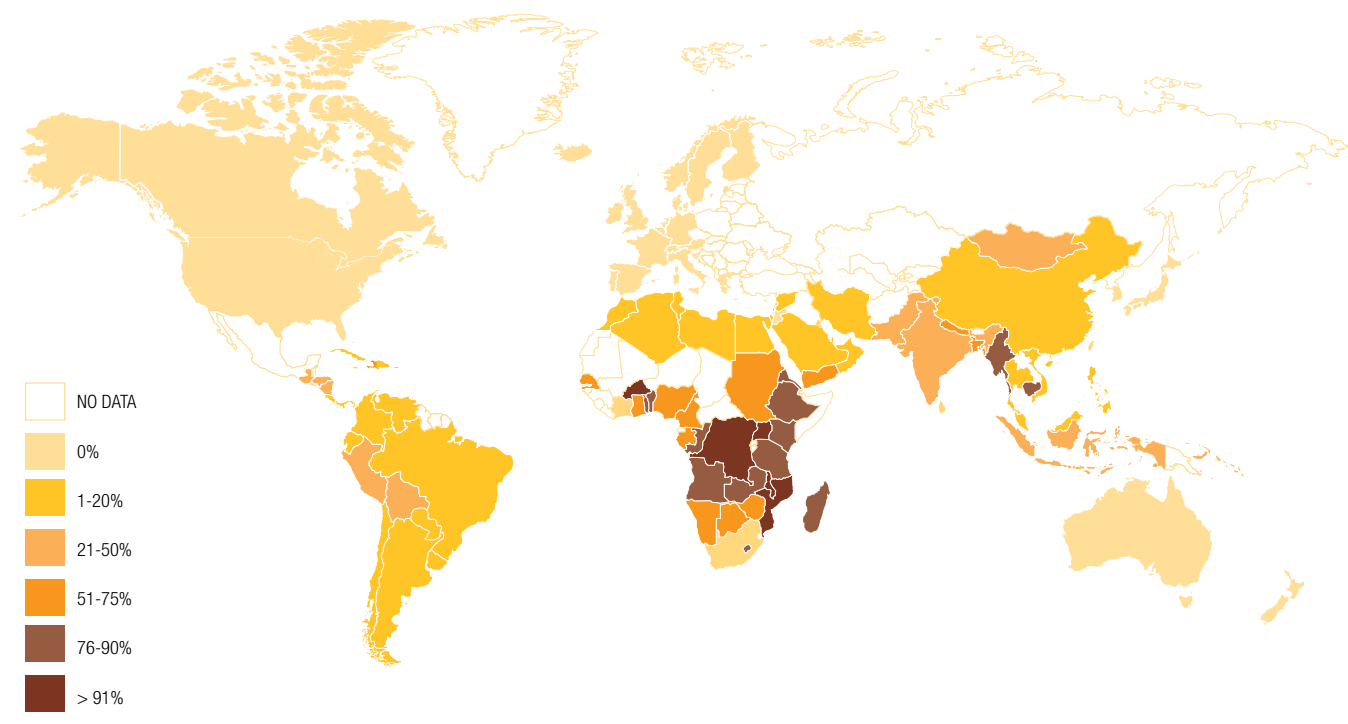

Figure 8.1 Pourcentage de la population privée d'électricité [Earthtrends 2009 (www.earthtrends. org) en reprenant les données du Human Development Report 2007/2008]

Ce sont traditionnellement les femmes et les filles qui ont la responsabilité de collecter le bois et d'aller chercher l'eau, ce qui exige du temps et des efforts physiques dépensés à d'autres occupations que l'école ou la production de revenu. De nombreuses femmes et fillettes souffrent aussi de problèmes de santé dus au fait de récolter le bois et de brûler de la biomasse. Du fait qu'elles cuisinent à l'intérieur sur des réchauds qui sont mal ventilés, les femmes sont exposées à toute une variété de risques sanitaires comme des infections respiratoires, des cancers et des maladies des yeux. La fumée des foyers mal ventilés est aussi responsable de près de deux millions de naissances prématurées par an. Le fait de remplacer des combustibles de mauvaise qualité comme la biomasse traditionnelle par des combustibles plus efficaces comme le kérosène, le gaz naturel, les biocarburants modernes ou l'électricité peut faire beaucoup pour réduire les impacts de la fumée et de la fatigue physique qui affectent la santé des femmes et des fillettes de façon disproportionnée (PNUD, 2004).

Il faut donc étudier les options en matière d'énergie par rapport aux coûts et bénéfices environnementaux et humains lorsque l'on fixe les critères orientant le choix des nouvelles formes d'énergie. De nombreuses options sont envisagées pour un avenir sans pétrole, certaines plus durables que d'autres. Toutes les sources d'énergie ont des impacts sur l'environnement, mais il est important d'évaluer la totalité des coûts et des bénéfices pour favoriser les options les plus équitables, les plus efficaces et les plus durables. Pourtant, seules les sources d'énergie qui dépendent d'une récolte durable de ressources environnementales ont la possibilité d'être vraiment renouvelables, et il faut concentrer tous les efforts pour augmenter le rôle que peut jouer l'environnement, tout en reconnaissant ses limites.

L'UICN a appelé tous les donateurs et parties prenantes à fournir le support nécessaire au développement et à la mise en œuvre de systèmes énergétiques écologiquement durables, socialement équitables et économiquement rentables, afin de soutenir le développement durable (Résolution 4.081 CMN/UICN).

Etant donné les défis de la demande énergétique croissante, alors que nous subissons des impacts 
toujours plus forts des changements climatiques, nous devons travailler d'urgence à la transition vers notre avenir énergétique. D’abord et surtout, nous devons encourager la conservation de l'énergie, dans tout plan de conservation et toute nouvelle approche en matière d'énergie. Chacun de nous doit faire des efforts pour conserver l'énergie - éviter tout voyage inutile, éteindre les lumières, l'air conditionné, les ordinateurs et les autres appareils électriques lorsque nous ne nous en servons pas, etc. Mais en plus, nous devrons explorer d'autres options. Nous devrons investir dans des évaluations stratégiques environnementales et sociales plus complètes des différentes options énergétiques, notamment des analyses coûts/bénéfices précises. Comme nous le faisons pour les biocarburants et les éoliennes, nous devons encourager le développement et la mise en ouvre de lignes directrices pour toutes les options énergétiques, en fonction de leurs impacts environnementaux. Le développement durable est à ce prix. 

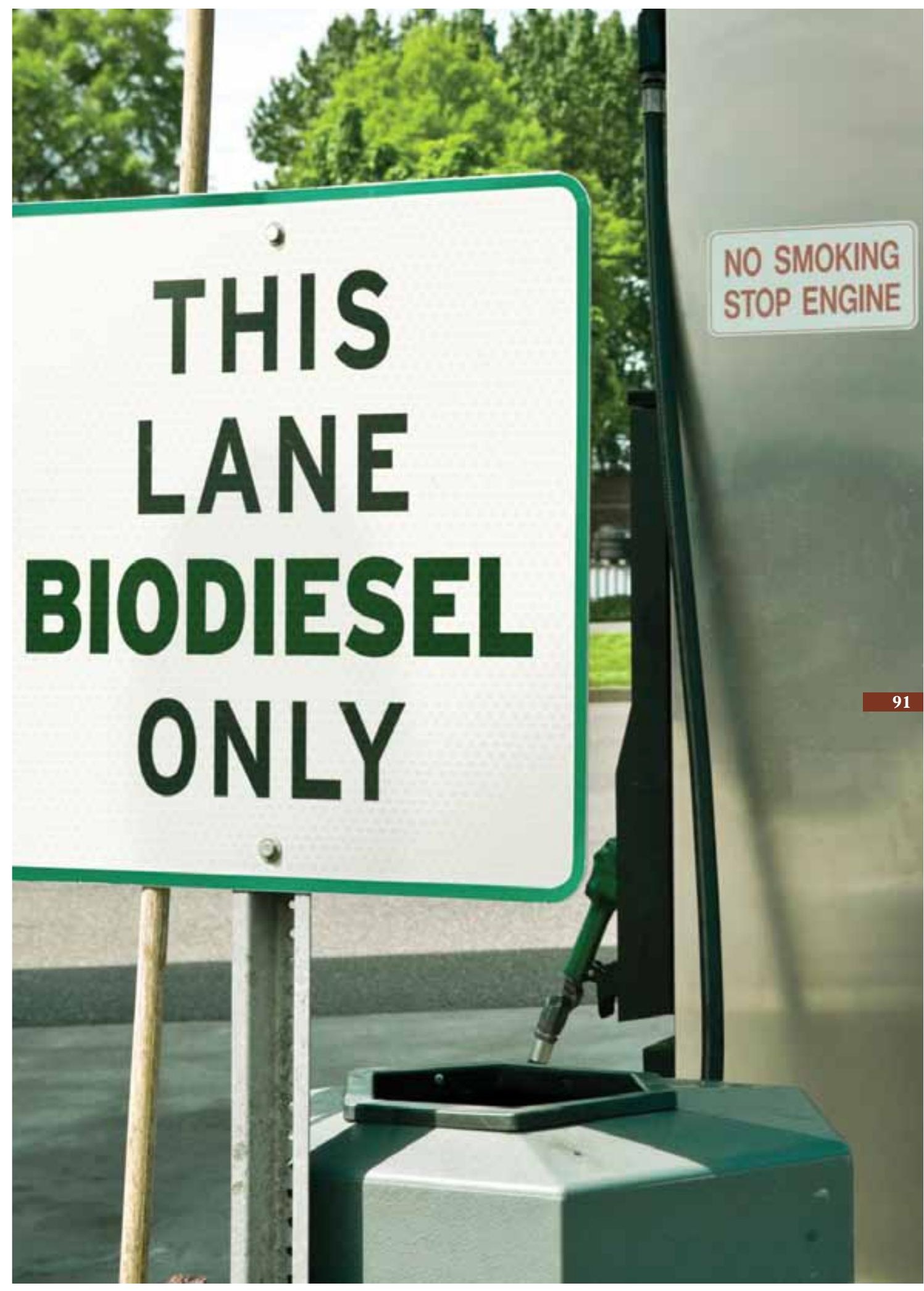


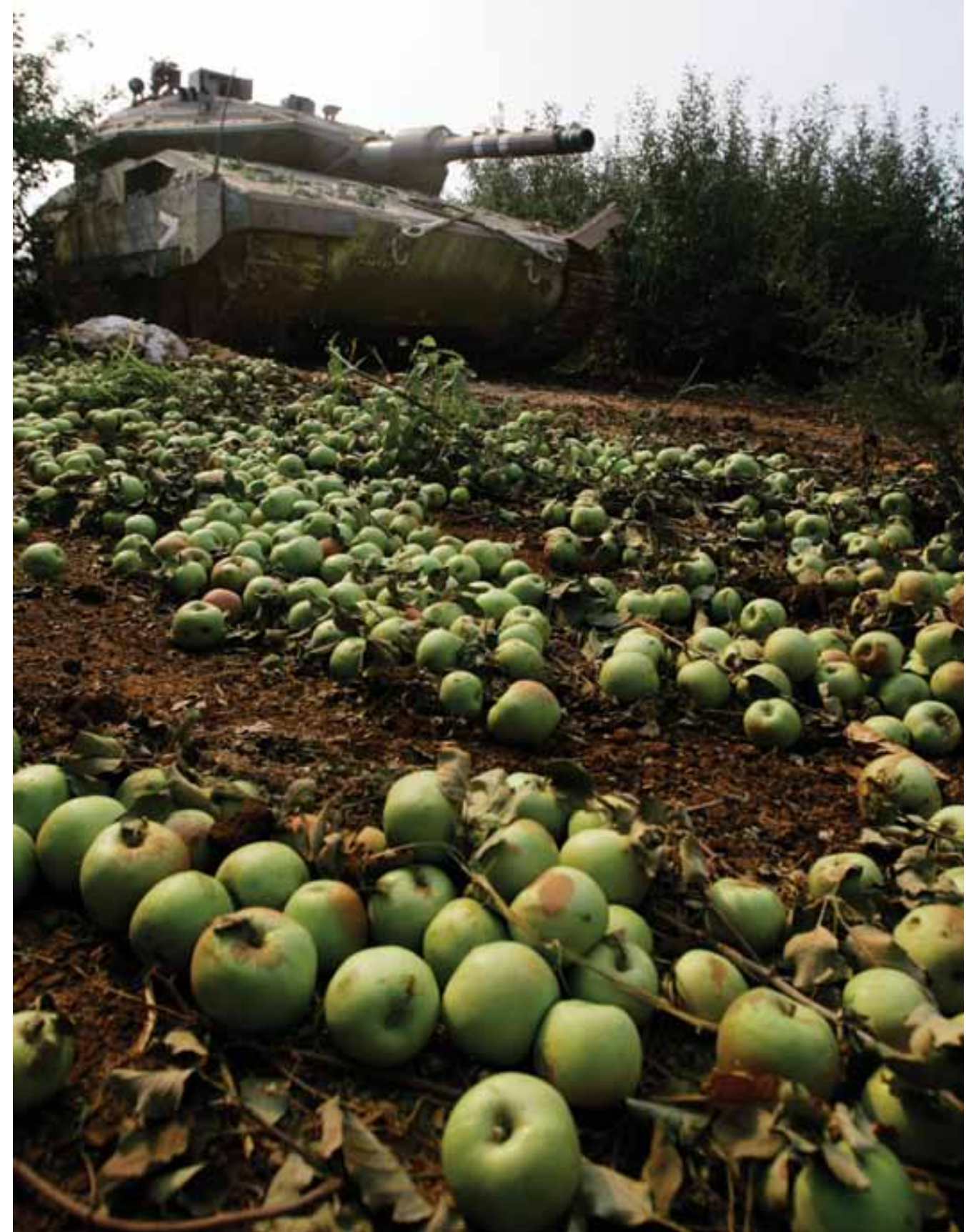


La guerre - conflit armé entre entités rivales - a des impacts importants sur les sociétés humaines impliquées et sur la biodiversité. Certes, ce sont les souffrances humaines qui sont les plus inquiétantes, mais les aspects écologiques des guerres et des reconstructions post-conflits méritent une attention certaine. L'UICN est active dans de nombreuses zones de conflits, elle y acquiert beaucoup d'expérience en s'occupant des problèmes de conservation dans les conditions difficiles de pays comme le Népal, le Pakistan, la Somalie, le Soudan, le Sri Lanka et la République Démocratique du Congo.

Aujourd'hui, les conflits armés sont particulièrement actifs dans des régions importantes pour la biodiversité. Plus de $80 \%$ des principaux conflits armés de la seconde moitié du $20^{\text {ème }}$ siècle se sont déroulés dans des hauts-lieux (hotspots) de la biodiversité, des régions qui abritent les populations entières de plus de la moitié de toutes les espèces végétales et de plus de $42 \%$ de tous les vertébrés. Deux-tiers des 34 hauts-lieux mondiaux ont connu la guerre pendant cette période (Hanson et al., 2009). Les hauts-lieux sont aussi particulièrement menacés parce que la pauvreté des pays où ils se trouvent en grande partie exerce une pression énorme sur les ressources de l'environnement naturel. La guerre dans ces hauts-lieux rend la conservation encore plus difficile dans la mesure où les réfugiés chassés par les combats se tournent souvent vers les forêts pour y trouver nourriture et matériaux de construction, ce qui ajoute à la pression sur la biodiversité.

Les guerres dans des habitats importants pour la faune sauvage ont touché de nombreux pays depuis les années 1990. Une liste incomplète reprend l'Angola, la Bosnie, la République Démocratique du Congo, le Cambodge, la République centrafricaine, la Colombie, le Guatemala, l'Inde, l'Indonésie, le Liberia, le Mexique, le Myanmar, le Népal, le Pakistan, le Pérou, les Philippines, la Sierra Leone, le Sénégal, le Sri Lanka, les Iles Salomon, et le Soudan - un catalogue bien déprimant. Ces conflits généralement civils se passent souvent dans des régions situées loin du contrôle du gouvernement, où peu de services publics sont disponibles pour les millions de personnes qui vivent dans ces régions reculées.

\section{CONFLITS ET BIODIVERSITÉ}

Les ressources naturelles peuvent être un facteur important dans les conflits, spécialement les conflits civils. Une analyse de 47 guerres civiles a révélé que le facteur qui annonce le plus sûrement une guerre civile est le niveau de dépendance vis-à-vis de l'exportation de 
Encadré 9.1 Etudes de cas de guerre civile et faune sauvage

\section{En Inde, les Naxalites et les tigres}

L'Inde est confrontée à des problèmes

d'insurrection croissants, particulièrement

parce que des tribus rebelles se sont retournées

contre le gouvernement et soutiennent

des groupes comme la guérilla maoïste des

Naxalites. Les Naxalites sont une menace pour la restauration des tigres en Inde parce qu'ils contrôlent de vastes régions de forêts reculées au centre et dans l'est de l'Inde - régions qui sont un habitat idéal pour les tigres. Même s'ils ne visent pas particulièrement les tigres, ils empêchent les activités de conservation dans les régions qui sont sous leur contrôle et qui peuvent représenter quelque $30 \%$ de l'aire de répartition du tigre en Inde. Et même si le tigre n'est pas une cible, les prélèvements excessifs de proies du tigre, comme les cerfs et les sangliers sauvages, forcent les tigres à se rabattre sur des animaux domestiques (voire des hommes) et à entrer en conflit avec les communautés rurales.

\section{Les rebelles philippins encouragent la conservation}

Aux Philippines, les rebelles de Mindanao ont menacé de s'en prendre aux bûcherons si le Gouvernement ne mettait pas fin à l'exploitation des arbres qui menace d'annihiler le couvert forestier de l'île. En février 2005, un porte-parole du Front National Démocratique a annoncé que les rebelles participaient à la conservation de l'environnement en lançant des campagnes de reforestation et d'éducation parmi les populations rurales pour soutenir l'utilisation durable des produits forestiers et pour réduire le plus possible les effets négatifs des cultures itinérantes. Ils continuent à soutenir que ces activités aident à préserver l'habitat sauvage de nombreux oiseaux et mammiferes endémiques de Mindanao, et ils prouvent que la conservation est l'affaire des gens, pas seulement la responsabilité des gouvernements. marchandises comme des grumes, des minerais ou du pétrole, des ressources que l'on peut qualifier de " pillables " (Collier, 2003). Cellesci peuvent fournir suffisamment de fonds pour soutenir un mouvement armé qui permettra aux vainqueurs de continuer à exploiter ces ressources. Le conflit est aussi souvent lié à des factions politiques et ethniques qui peuvent être en conflit pour d'autres raisons, mais la motivation première, au moins dans certains cas, est la volonté de retirer des bénéfices financiers de l'exploitation des ressources. Un seul exemple : la vente de grumes en provenance des zones qui sont aux mains des rebelles au Liberia permet aux insurgés de se fournir en armes et de continuer à gagner un revenu, et les bénéfices qu'ils retirent en prolongeant la guerre les dissuadent peut-être de rechercher la paix.
Les impacts des conflits sur la biodiversité comprennent des modifications de la distribution des personnes et des espèces sauvages, des changements éventuels des schémas d'exploitation et l'amplification d'autres problèmes, notamment de la pauvreté (Encadré 9.1).

Les schémas des guerres influencent depuis longtemps la distribution des espèces et des écosystèmes, et plus généralement de la biodiversité. Certains grands mammiferes sont particulièrement vulnérables en période de conflits. Le rhinocéros blanc, par exemple, fut exterminé du Soudan pendant la guerre civile (1955-1972), et la guerre entre l'Ouganda et la Tanzanie a pratiquement éradiqué le rhinocéros noir de ces deux pays. 
La guerre civile qui a commencé en République Démocratique du Congo en 1996 a eu un coût humain tragique, avec plus de 3,8 millions de morts, et elle a forcé les agriculteurs à quitter leurs terres et à devenir des braconniers ou des réfugiés errants, qui se sont souvent installés autour, voire à l'intérieur de parcs nationaux. Malgré l'Accord de paix de 2003, le Parc National des Virunga est encore occupé par des hommes armés qui braconnent librement le gibier, pour le consommer ou le vendre. Dans ces conditions, le commerce de viande de brousse en République

Démocratique du Congo est étroitement lié au secteur informel au sens plus large. Merode et Cowlishaw (2006) ont récolté des informations sur la vente d'espèces protégées et non protégées sur les marchés urbains et ruraux, et sur la chaîne des produits de viande de brousse qui alimentent ces marchés en période d'instabilité politique et de conflit armé. En temps de paix, la viande des espèces protégées du Parc National de la Garamba (surtout éléphants et buffles) est peu présente sur les marchés ruraux mais elle constitue plus de la moitié de toutes les ventes de viande de brousse sur les marchés urbains. Le schéma reflète bien la différence entre les chaînes des produits qui alimentent les marchés ruraux et urbains. Il faut des armes automatiques pour chasser les grandes espèces protégées et ce sont des officiers militaires, qui contrôlent les marchés urbains, qui les fournissent aux chasseurs. Les chefs traditionnels, qui administrent les marchés villageois, désapprouvent l'usage de ces armes. En temps de guerre, les ventes d'espèces protégées sur les marchés urbains se sont multipliées par cinq parce que les officiers militaires ont fui la scène, laissant derrière eux un système d'accès libre qui a entraîné une augmentation massive de l'exploitation des espèces protégées. Par contre, les marchés ruraux sont restés relativement stables parce que l'autorité des chefs de village s'y est maintenue. Ces résultats laissent entendre que, même en temps de conflits violents, les autorités traditionnelles peuvent jouer un rôle important dans la conservation de la biodiversité.

La suite continue de guerres en Afrique centrale a un impact négatif sur les populations et sur des espèces sauvages comme les hippos. Dans le lac Edouard, à la frontière entre l'Ouganda et la République Démocratique du Congo, par exemple, la population d'hippos a décliné de 9600 dans les années 1970 à près de 680 en 2005, à cause du braconnage exercé par les milices insurgées qui voulaient de la viande. L'impact

écologique de ce déclin est profond, parce que la population saine des hippos déversait par an dans le lac, par ses excréments, près de 100 millions de tonnes de nutriments qui nourrissaient un phytoplancton microscopique, qui lui-même servait de nourriture aux vers et aux larves aquatiques, aliments des tilapias du lac qui étaient pêchés par plusieurs milliers de pêcheurs qui vivaient dans le Parc National des Virunga. Le stress du déclin des poissons est aggravé par la demande croissante en tilapias, et le nombre de gens qui utilisent des filets aux mailles plus fines augmente rapidement. Cela signifie que les poissons sont attrapés de plus en plus jeunes et que le recrutement chute rapidement, obligeant les gens à se tourner vers la faune sauvage comme source de protéines. Si cela est désastreux pour les pêcheurs et pour la faune sauvage, ce n'est peut-être pas si mal pour le lac Edouard, parce 
que le tilapia n'est pas une espèce indigène et que son épuisement pourrait permettre aux espèces indigènes endémiques de poisson - dont beaucoup restent encore non documentées - de se rétablir. Le plus sérieux problème concerne la faune sauvage du Parc National des Virunga qui borde le lac Edouard et qui est menacé par les conflits armés et par les effets secondaires de l'installation de réfugiés.

La faune sauvage peut répondre en allant vers des habitats moins risqués. L'histoire montre que, dans de nombreuses régions du monde, les zones tampons entre des groupes ethniques qui peuvent entrer en conflits sont parfois particulièrement riches en biodiversité, sans doute en partie parce que, par crainte de conflit avec les autres groupes ethniques, elles sont moins victimes de la pression de la chasse. Par exemple, une étude réalisée au sud-Soudan par la Wildlife Conservation Society en 2007 a découvert ce qui pourrait bien être la plus grande migration de mammiferes du monde : plus de 1,3 millions de cobes à oreilles blanches prospèrent dans la région qui entoure le Sudd, la plus vaste zone humide d'eau douce en Afrique. L'étude a aussi estimé la population d'éléphants à 8000 , les rhebucks à 13.000, les buffles à 8900 et les lechwes du Nil (une espèce qui ne se trouve que dans cette région) à près de 4 000. Le Sudd est resté sous-développé à cause de la guerre civile du Soudan, mais de nombreux intérêts jettent un regard gourmand sur la région, reconnaissant en elle un grenier potentiel pour l'Asie de l'Ouest, mais aussi pour la Chine et l'Afrique de l'Ouest.

\section{PÉRIODE POST-CONFLIT ET BIODIVERSITÉ}

La paix post-conflit peut en réalité poser plus de problèmes à la conservation que le conflit lui-même. Lorsque les combattants cessent de se battre, les zones qui étaient auparavant hors d'atteinte à cause de la guerre deviennent des sites de choix pour le développement, entraînant déforestation, braconnage de la faune et autres formes de dégradation. Si certaines aires protégées ont été créées, la biodiversité risque du souffrir davantage après la guerre que pendant (par exemple, en Angola et au Mozambique). De plus, les armes qui se sont multipliées pendant la guerre circulent et, à en juger par l'expérience passée, la paix pousse au moins certains des combattants à devenir braconniers, pressés de convertir la faune sauvage en viande, comme moyen de subsistance en ces temps de grande incertitude.

Plus surprenant encore, quand la guerre prend fin, les biologistes de terrain peuvent faire de nouvelles découvertes. Par exemple, quand les scientifiques sont retournés dans les forêts du Vietnam après la guerre, un nombre incroyable de nouvelles espèces furent découvertes par les biologistes de terrain travaillant pour des organisations membres de l'UICN, comme la Wildlife Conservation Society. Ces découvertes comprennent le muntjac géant, de loin le plus grand des cerfs aboyeurs, le saola, une antilope de forêt si différente qu'elle s'est vu attribuer un nouveau genre, un nouveau genre aussi et une nouvelle espèce de chèvre de forêt connue localement sous le nom de Linh duong, des preuves de l'existence d'au moins deux nouvelles espèces de cerfs, et un suidé sauvage qui n'avait plus été vu depuis 100 ans. Les activités de l'UICN en RPD Lao, au Vietnam et au Cambodge contribuent à la conservation post-conflit.

\section{EMPÊCHER UN CONFLIT ET ATTÉNUER SON IMPACT SUR LA BIODIVERSITÉ}

La sécurité nationale est un problème récurrent, et les risques pour certains gouvernements sont bien réels même s'ils peuvent prendre des formes inattendues. Une réponse visant à anticiper un conflit armé est la création de parcs internationaux pour la paix et d'aires protégées 


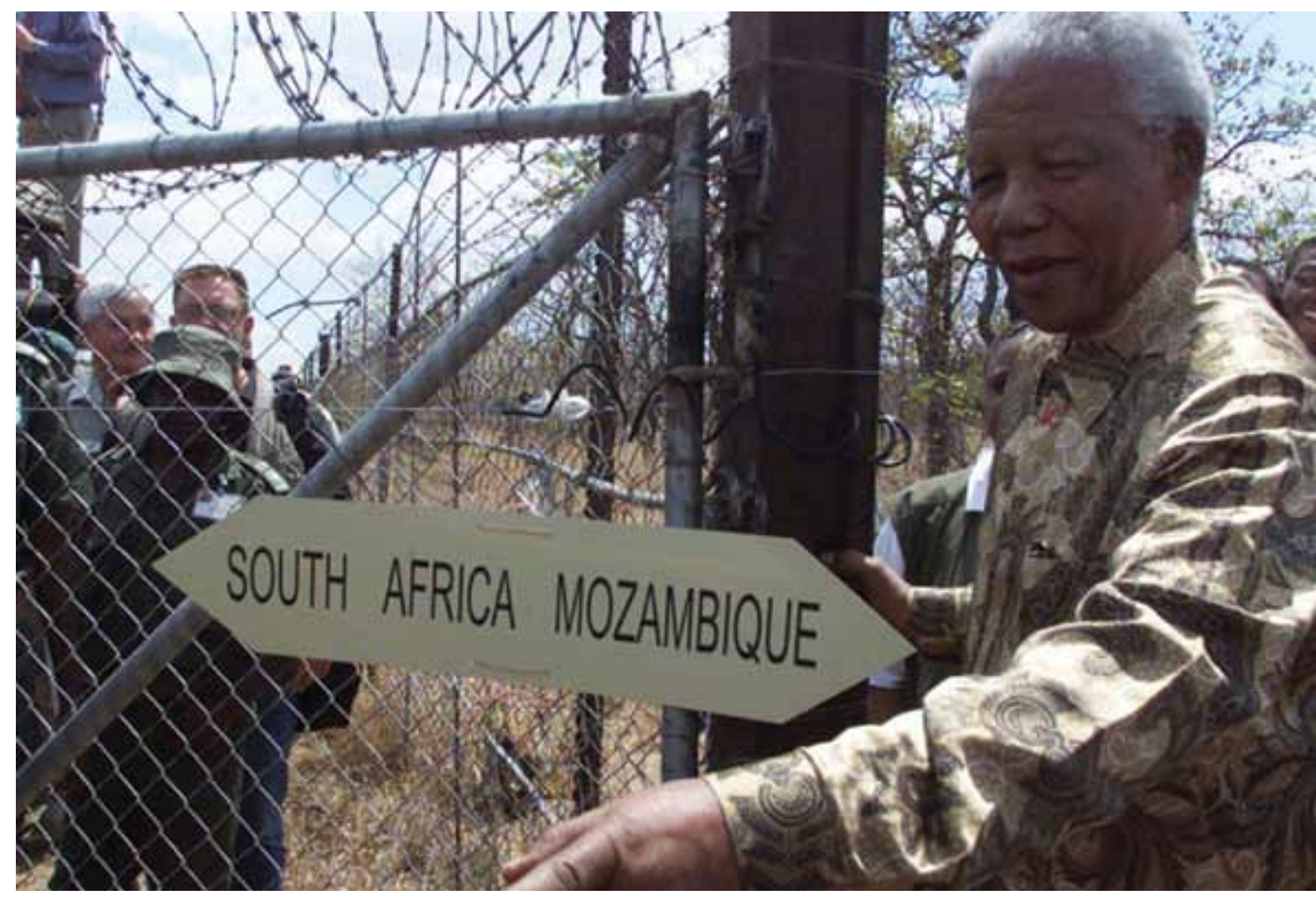

transfrontalières qui requièrent davantage de coopération avec les pays voisins. Par exemple, l'Afrique australe comprend au moins quatre aires protégées transfrontalières: Kgalagadi entre l'Afrique du Sud et le Botswana, MalotiDrakensberg entre l'Afrique du Sud et le Lesotho, Great Limpopo partagé entre l'Afrique du Sud, le Mozambique et le Zimbabwe, et Nyika entre le Malawi et la Zambie. De telles aires protégées posent de nouveaux défis et offrent de nouvelles opportunités.

Une meilleure compréhension des causes et des conséquences des conflits et de l'écologie des guerres peut permettre aux organisations de conservation de continuer à fonctionner même pendant les conflits armés, comme ce fut le cas récemment au Népal. Certaines aires protégées, comme la magnifique Aire de conservation du Kangchenjunga, furent par exemple confiées aux communautés locales pour qu'elles les gèrent, et la chasse locale des animaux sauvages pour leur viande fut notablement réduite au moins dans certaines d'entre elles. De nombreux projets de l'UICN au Népal ont pu continuer pendant le conflit, ce qui leur a permis d'être tout à fait opérationnels dès que la paix est revenue.

De nombreux conflits surviennent dans des régions frontalières qui sont souvent éloignées $\mathrm{du}$ gouvernement central. Ces régions sont aussi souvent riches en faune sauvage. La possibilité de créer des aires protégées transfrontalières comme moyen d'encourager la paix est de plus en plus populaire. Les parcs pour la paix n'ont rien de nouveau, ils servent de zones tampons entre des gouvernements qui sont par ailleurs en conflit. Le Réseau mondial des aires protégées transfrontalières a identifié 227 complexes transfrontaliers d'aires protégées qui totalisent près de 3043 aires protégées individuelles ou sites désignés couvrant 4,6 millions de kilomètres carrés (GTPAN, 2009). Ces parcs dits « pour la paix » peuvent instaurer la routine d'une 


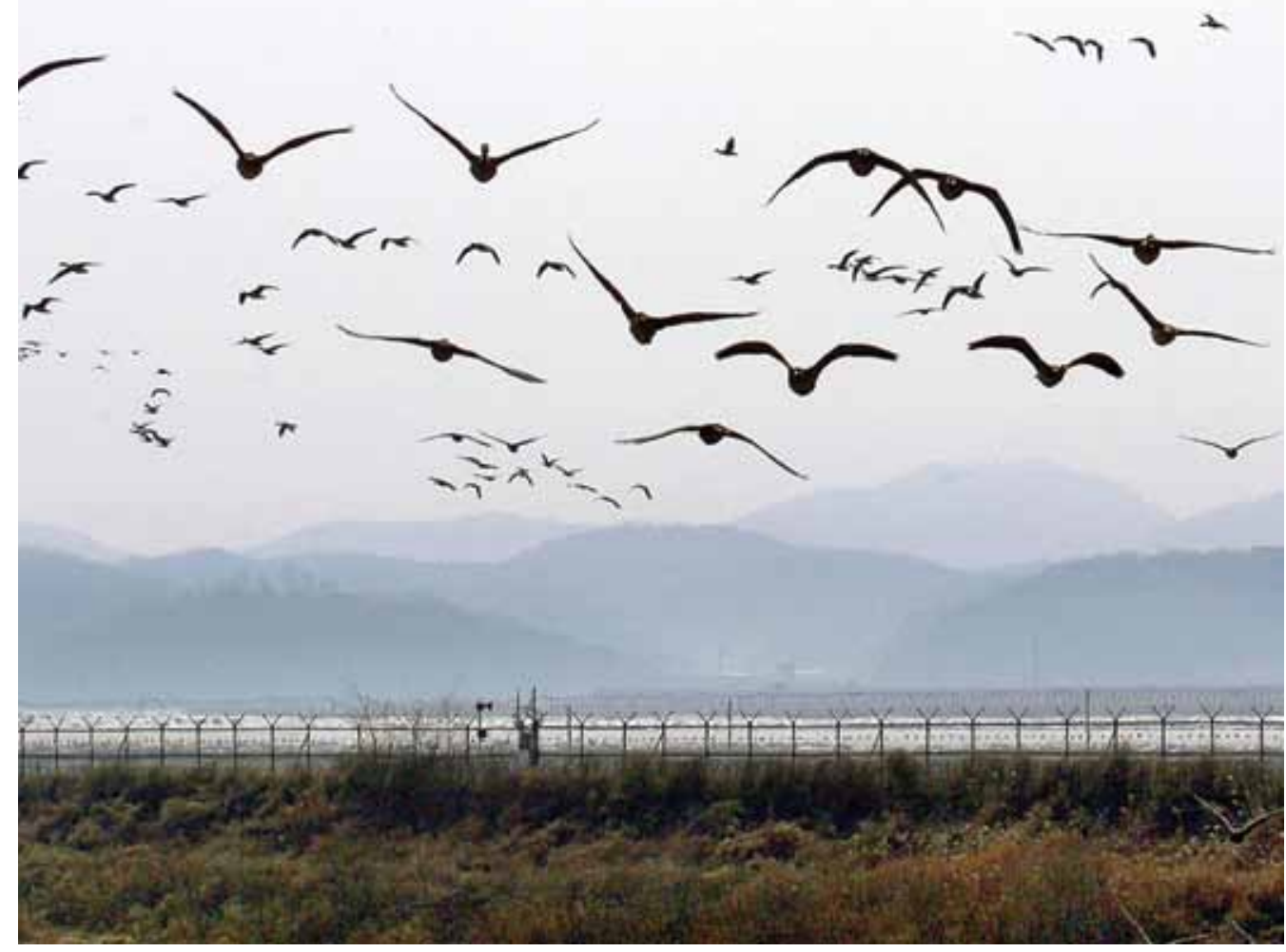

coopération internationale, promouvoir les identités et les intérêts régionaux, réduire la probabilité des conflits, étendre la superficie des habitats naturels pour la faune et ils donnent l'espoir que la conservation peut aider à apporter la paix aussi bien aux hommes qu'à la nature.

Les armées restent une force politique, sociale et économique dominante dans de nombreux pays. Les armées modernes reconnaissent de plus en plus que les viabilités politique, économique et écologique sont étroitement liées et qu'elles contribuent à la conservation dans de nombreux pays. Elles contrôlent de grandes portions de terres, terrains d'entraînement ou domaines militaires, et patrouillent souvent le long de frontières éloignées, importantes pour la biodiversité. On peut même arguer que de nombreuses menaces pour la sécurité nationale plongent leurs racines dans une gestion inappropriée des ressources naturelles (Klare, 2001) et on pourrait donc légitimement espérer que les militaires favorisent une meilleure conservation des ressources. Plusieurs Membres de l'UICN travaillent en ce sens avec des militaires.
Les gouvernements sont certainement au courant des risques que les conflits représentent pour la biodiversité. La Convention sur la diversité biologique (CDB), dans son Article 3, soutient la Charte des Nations Unies en reconnaissant la responsabilité des Etats « de faire en sorte que les activités exercées dans les limites de leur juridiction ou sous leur contrôle ne causent pas de dommage à l'environnement dans d'autres Etats ou dans des régions ne relevant d'aucune juridiction nationale ». Ceci établit une base légale claire pour éviter tout dommage environnemental lors de conflits violents entre gouvernements. La Convention souligne aussi la valeur de la paix pour la biodiversité, et conclut que « en fin de compte, la conservation et l'utilisation durable de la diversité biologique vont renforcer les relations amicales entre les Etats et contribuer à la paix pour toute l'humanité ».

Mais il reste d'importantes lacunes dans les lois internationales qui gouvernent et protègent l'environnement en temps de conflit armé, que 
ce soit au point de vue normatif ou administratif (l'une des moindres n'est pas la question de savoir comment mettre en pratique les exigences normatives). Il faut de nouvelles mesures pour combler ces lacunes. Des systèmes pour garantir les responsabilités et les compensations pour les transgressions contre l'environnement et les ressources naturelles pendant les conflits armés devraient inclure un tribunal spécial international pour étudier les plaintes en matière de dommages environnementaux et des études de cas sur l'impact environnemental de conflits armés.

Quelles sont les implications de tout cela pour des organisations de conservation, comme l'UICN et ses Membres ? S'ils sont impliqués dans la conservation à l'intérieur d'une zone de conflit, les conservationnistes devraient faire tout ce qui est possible pour maintenir une présence dans cette zone. Cela peut signifier qu'il faut travailler en passant par des organisations non gouvernementales (ONG) locales et éviter d'être considérés comme un outil du gouvernement mais plutôt comme des supporters des intérêts légitimes des populations qui vivent dans la zone de conflit. L'expérience de l'UICN en Amérique centrale, au Népal, en Inde et dans certaines régions d'Afrique montre que les groupes insurgés permettent souvent aux ONG de poursuivre les activités de conservation qui profitent aux communautés rurales. De plus, la communauté de la conservation doit continuer à soutenir le plus possible les organismes locaux de conservation. Cela peut signifier qu'elle s'implique lorsque le support du gouvernement se retire, et qu'elle aide le personnel de terrain local à garder de bonnes relations avec les populations locales dont la gestion des ressources est fondamentale pour la conservation.

Il est essentiel de chercher à comprendre objectivement toutes les plaintes exprimées depuis longtemps par les communautés qui vivent dans les zones reculées où les conflits sont endémiques, et d'utiliser ce que l'on a compris pour concevoir des formes d'appui appropriées aux intérêts de la conservation.

L'expérience des 30 dernières années montre qu'une gestion efficace des ressources naturelles peut favoriser le rétablissement de la paix post-conflit et la restauration ; par contre, le manque d'intérêt pour les ressources naturelles ou une gestion inefficace peuvent compromettre la paix dans les sociétés post-conflits. Donc, si les conflits et leurs conséquences sont indiscutablement un désastre pour les gens, ils ne doivent pas nécessairement l'être aussi pour la faune sauvage (McNeely, in press).

Pour aider les communautés dans les situations post-conflit, ceux qui travaillent dans les domaines de l'environnement, des conflits, et du rétablissement de la paix ne devraient pas susciter des attentes irréalistes de la part des communautés locales, parce que la gestion des ressources naturelles lors du rétablissement de la paix est à très long terme, lent et difficile. Les conservationnistes doivent aussi collaborer avec les agences humanitaires pour favoriser la réinstallation des réfugiés dans des zones favorables qui ne risquent pas d'endommager les valeurs de la conservation. 


\section{Affronter les catastrophes : \\ 10. réflexions sur la restauration post- catastrophe à l'échelle de l'écosystème}

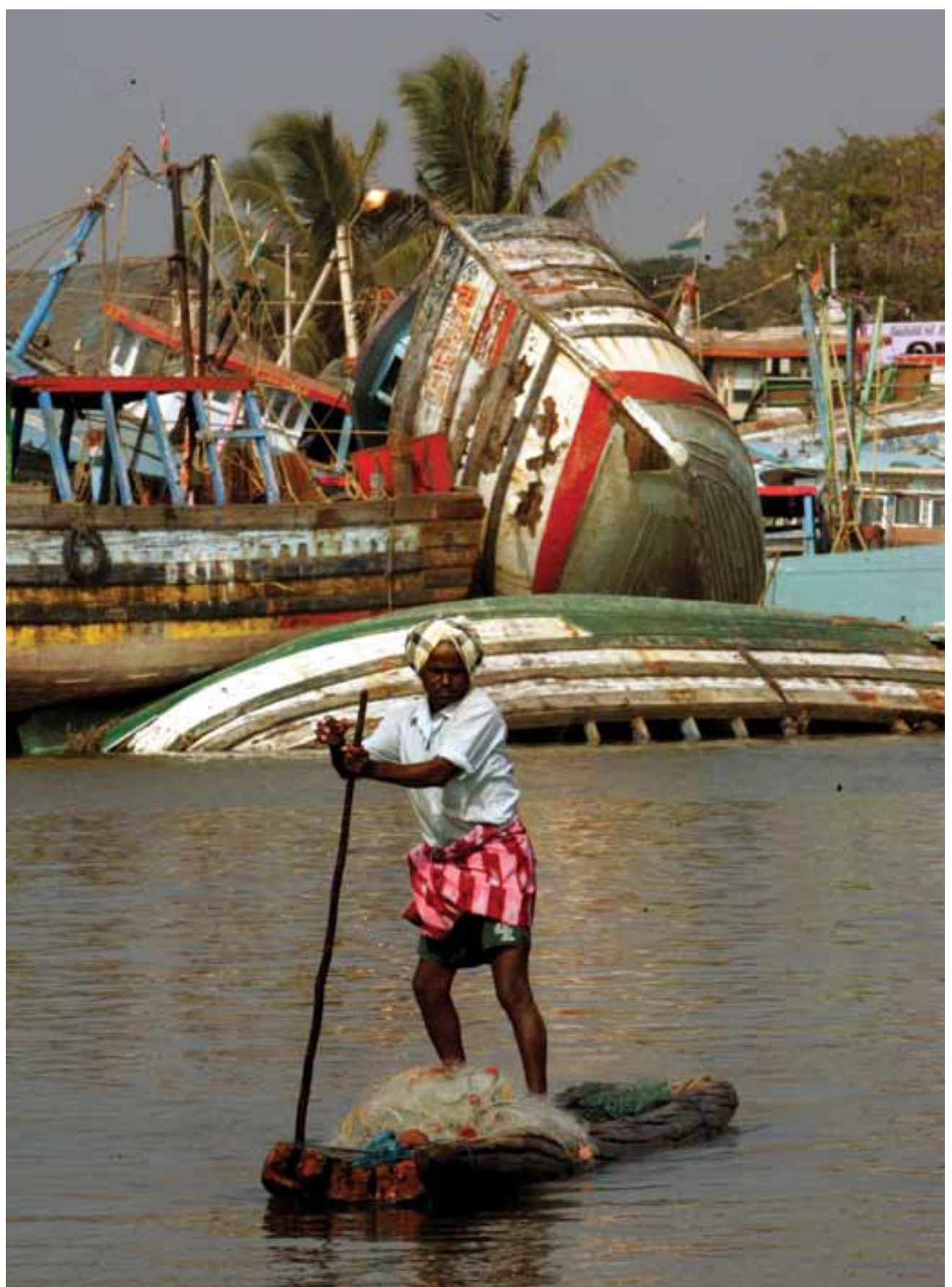


Ces dernières années, les événements naturels extrêmes - tremblements de terre, tsunamis et ouragans - semblent se faire plus fréquents, et ils tournent au désastre lorsque des intérêts humains sont affectés. L'impact croissant de ces catastrophes pourrait être dû à la population humaine croissante dans les régions vulnérables. Si importants et urgents que soient les besoins humains après les catastrophes, les efforts de rétablissement devraient aussi prendre en compte la façon dont la nature peut y contribuer. Les services écosystémiques (Chapitre 4) sont touchés par ces événements extrêmes mais ils peuvent apporter une partie de la réponse. Les efforts de reconstruction après les catastrophes sont souvent l'occasion d'introduire des changements plus importants et à plus long terme, qui vont aider au rétablissement des moyens de subsistance et de la sécurité des hommes, mais aussi des écosystèmes dont beaucoup dépendent.

Tout plan de rétablissement réussi doit comporter un volet qui se préoccupe des données environnementales. Cela va réduire la vulnérabilité des gens en cas de nouvelles catastrophes. L'histoire montre que les tremblements de terre ont tendance à se reproduire dans les mêmes régions et que des événements comme les inondations, les cyclones et les sécheresses deviennent plus fréquents et plus intenses. Forte de cet avertissement, la planification doit inclure toutes les mesures possibles pour compenser ou pour s'adapter à ce monde en changement. Ceux qui sont impliqués dans la reconstruction ont désormais la possibilité de modifier la façon dont ils répondent aux exigences de la reconstruction à long terme et d'établir des moyens de subsistance durables.
Mais pour exploiter au mieux cette possibilité, ils doivent être sûrs de tirer les leçons apprises lors des catastrophes précédentes et employer des stratégies appropriées en préparant leur réponse.

L'environnement doit être au centre des préoccupations à tous les stades de la réponse à une catastrophe classique. Au début, évidemment, la phase de sauvetage doit se focaliser sur les besoins immédiats des hommes touchés par l'événement. Mais l'impact possible des actions entreprises dans les phases de sauvetage et de secours pour soulager les souffrances humaines peut aussi avoir des effets négatifs imprévus sur l'environnement. Par exemple, le fait de fournir du bois de feu au lieu de kérosène ou de gaz naturel peut entraîner la déforestation de la région environnante et 
augmenter ainsi le risque de glissements de terrain. Les décisions prises pour des mesures à court terme, comme des installations temporaires, peuvent avoir des conséquences prolongées, par exemple si les réfugiés sont hébergés dans un parc national. Le rétablissement à long terme exigera normalement des actions concertées et bien dirigées, pour les hommes comme pour leur environnement

\section{PLANIFICATION DE RESTAURATION À COURT TERME ET ENVIRONNEMENT}

Deux activités clés de la phase de secours - la gestion des déchets et la réinstallation des personnes affectées - peuvent avoir une influence majeure sur les fonctions des écosystèmes. Causes directes de la dégradation de l'environnement, les deux peuvent entraîner la perte et la fragmentation d'habitats, la pollution et la dispersion d'espèces envahissantes. La réinstallation peut aussi avoir comme conséquence imprévue la surexploitation des ressources naturelles étant donné que les personnes qui vivent dans de nouveaux endroits auront probablement besoin de se débrouiller pour trouver à manger, pour trouver du bois pour le chauffage et la cuisson, et d'autres produits naturels à manger ou à vendre pour en tirer quelque profit.

Pour une future planification, on peut tirer d'importantes leçons de certains exemples spécifiques, de l'expérience de réponses données à des catastrophes majeures. Par exemple, la Banque asiatique de développement (BAsD), dans un examen de la restauration d'Hambantota, dans la mer d'Andaman, au Sri Lanka, après le tsunami de 2004, a remarqué que les débris accumulés dans les lagons et les autres écosystèmes côtiers, plus l'infiltration de sel à l'intérieur des terres, avaient eu des effets négatifs sur la pêche locale et sur la productivité agricole. Le rapport indiquait aussi que les actions de nettoyage avaient affecté l'environnement parce que les municipalités jetaient les déchets dans les zones humides, avec un impact négatif sur les systèmes de drainage et les zones de rétention des eaux, augmentant par là-même les risques de maladies véhiculées par l'eau. Certains sites de réinstallation proposés menaçaient des zones riches en biodiversité, de sorte que le rapport a recommandé de les situer plutôt à une certaine distance des sites qui contenaient une biodiversité fragile, avec une zone tampon entre les deux, et de s'assurer que le nombre de ménages réinstallés corresponde à la capacité de charge de la région

(BAsD, 2005).

Les espèces

envahissantes sont une autre menace importante, mais celle-ci est souvent négligée. Une évaluation environnementale post-tsunami réalisée par le Programme des Nations Unies pour l'environnement(PNUE) et le Ministère sri-lankais de l'Environnement et des Ressources naturelles a révélé, entre autres, que les vagues géantes avaient amené des espèces exotiques comme les figues de Barbarie (Opuntia) et le mesquite tolérant au sel (Prosopis) à l'intérieur des terres et atteint des aires protégées comme le Parc National de Yala. Ces espèces non indigènes sont en train de remplacer les espèces natives qui sont pourtant plus appréciées par le bétail et la faune sauvage du Sri Lanka (PNUE, 2005).

Les espèces envahissantes furent aussi un sujet d'inquiétude pour les plans de reconstruction suite à l'ouragan Katrina qui avait frappé la 
Nouvelle-Orléans en 2006. Le termite souterrain de Formose (Coptotermes formosanus) est originaire de Chine mais il fut introduit accidentellement aux États-Unis et il a depuis envahi au moins neuf états du Sud. Avant l'ouragan Katrina, le termite de Formose était responsable de dommages dans les maisons et les usines de la région de la Nouvelle-Orléans, estimés à 100 millions de dollars par an (US EPA, 2005). Après Katrina, le Département de l'Agriculture et des Forêts de Louisiane a fait passer le Formosan Termite Initiative Act, qui est en fait une mise en quarantaine des débris du désastre (Département de l'Agriculture de Louisiane, 2005). La loi notait que « l'ouragan a laissé des millions de tonnes de débris de bois, y compris des bois infestés par des termites de Formose » et que "l'instauration de cette quarantaine est indispensable pour prévenir la dispersion des termites de Formose et l'infestation de zones, de maisons et de structures qui ne sont actuellement pas infestées ou qui doivent être construites ou réparées".

Une des priorités, à court terme, est de gérer les déchets et les décombres. Dans le cas du tremblement de terre au centre de la Chine en 2008, la question des déchets est hallucinante. Il paraît que l'armée chinoise a dégagé plus de 8 millions de mètres cubes de décombres du tremblement de terre, assez pour remplir 16 fois l'intérieur du « Nid d'oiseau », le Stade olympique de Beijing (WRI, 2008). De plus, les soins donnés au grand nombre de blessés ont produit d'importantes quantités de déchets médicaux qui nécessitaient un traitement méticuleux. Enfin, le centre de la Chine est un gros producteur de nombreux produits chimiques destinés à l'agriculture, et plusieurs rapports d'évaluation des dommages ont signalé la dispersion de produits chimiques tels que du phosphore et l'ammoniaque dans l'environnement (RSC, 2008).

Pris ensemble, les déchets médicaux et les gravats, auxquels s'ajoutent les fuites des sites chimiques, constituent autant de risques potentiels pour l'environnement dont les gens dépendent pour leur nourriture, l'eau et les abris. Si l'on ne s'occupe pas rapidement de ces menaces sur les services écosystémiques, à long terme c'est le rétablissement des moyens de subsistance des populations qui s'en trouvera retardé.

\section{PLANIFICATION DE RESTAURATION À PLUS LONG TERME}

Une planification de la restauration à plus long terme, qui tient compte des préoccupations environnementales, est une approche tout à fait rentable. Lorsque la première planification commence, trois éléments clés doivent guider les considérations environnementales les connaissances, les capacités et le support politique.

\section{Connaissances}

Il est important de bien comprendre les impacts sur l'environnement, et donc sur les services écosystémiques fournis, pour planifier et constituer une base face à laquelle mesurer l'efficacité des activités de restauration. Les impacts des événements sont souvent ressentis bien au-delà de la zone directe de l'impact, et les évaluations d'impact doivent en tenir compte. Par exemple, il est possible que les emplois de certaines personnes dépendent de ressources qui se trouvent dans une région touchée, même s'ils habitent à des centaines de kilomètres de là.

Toute action efficace nécessitera non seulement une base de connaissances solide quant à l'état de l'environnement quand l'action commence, mais aussi un suivi à long terme des changements de l'écosystème pour favoriser une gestion adaptative.

\section{Capacités}

La capacité de réagir à toute catastrophe est une des clés du succès. La seule ampleur de la plupart des catastrophes implique que toutes 
les personnes disponibles sont nécessaires. La réhabilitation de l'écosystème exige le soutien des personnes qui vivent dans la région et, souvent, d'un financement gouvernemental des agences de coopération. Les capacités comprennent aussi le support et l'engagement de tous dans les activités de restauration. Il faudra aussi faire des campagnes de sensibilisation du public à l'importance du rôle des écosystèmes dans la restauration.

\section{Support politique des activités}

De catastrophes passées, on a déjà tiré plusieurs leçons sur le besoin de politiques favorables, qui viennent en aide à la gestion de l'environnement à long terme. Par exemple, il faut des politiques en matière de code de construction des bâtiments, qui définissent le zonage des types de construction et les matériaux utilisés; et il faut évidemment rendre obligatoires les exigences de l'Etude d'impact environnemental (EIE) en matière d'infrastructures, à court et à plus long terme. De plus, comme les catastrophes peuvent gravement altérer l'environnement de plusieurs régions, la réhabilitation peut exiger la création et le maintien d'aires protégées en guise de zones tampons.

En préparant des plans à long terme, il faudrait envisager plusieurs politiques comme :

- Les politiques en matière d'écosystème qui encouragent une hétérogénéité spatiale et biologique lorsqu'elles choisissent des sites et qui améliorent la résilience écologique en rétablissant les processus écologiques dont les communautés agricoles et naturelles dépendent (ex. cycles de l'eau, des nutriments, des débits) ;

- Les politiques socioéconomiques qui favorisent le développement d'infrastructures qui minimisent l'impact sur les écosystèmes, créent des ressources nouvelles et éventuellement renouvelables, et ajoutent à la diversité des ressources économiques disponibles.
Lorsque les connaissances, les capacités et les politiques sont acquises, les actions efficaces peuvent suivre. Il faut les aborder avec, à l'esprit, une approche à l'échelle du paysage. La gestion à cette échelle est suffisamment vaste pour reconnaître le rôle de tous les facteurs et des parties prenantes critiques qui influencent les décisions en matière d'aménagement du territoire (McNeely et Scherr, 2003 ; Scherr et McNeely, 2007). Une bonne gestion du territoire peut satisfaire les besoins sociétaux en équilibrant équitablement les compromis entre les exigences de production et les exigences sociales et environnementales de l'utilisation actuelle du territoire.

Les approches par paysage doivent inclure une attention spécifique pour le flux environnemental des eaux. Un flux environnemental est l'eau produite dans un cours d'eau, une zone humide ou une zone côtière et qui sert à préserver les écosystèmes et leurs avantages (Dyson et al., 2003). L'aménagement des ressources en eau en amont d'un cours d'eau a des conséquences sur les moyens de subsistance des personnes et sur les écosystèmes en aval. Des flux environnementaux corrects contribuent de façon critique à la bonne santé du cours d'eau, au développement économique et à la réduction de la pauvreté. Ils garantissent la disponibilité constante des nombreux bénéfices que les cours d'eau en bon état et les systèmes souterrains procurent à la société. Ceci est crucial pour l'atteinte de l'Objectif 7 du millénaire pour le développement (OMD) (durabilité environnementale) et, de manière indirecte, cela participe à l'atteinte des OMD liés à la santé et à l'éducation.

En préparant les plans de restauration des écosystèmes, il faut envisager plusieurs options. De nombreuses parties prenantes différentes sont impliquées, des villageois qui sont sur place, des citadins qui dépendent des produits de la région mais aussi des touristes internationaux qui 
apportent des revenus importants. Chacun peut vouloir quelque chose de différent, et les choix de développement devront équilibrer ces demandes. Il existe des outils pour aider à mener à bien cet exercice, pour développer des scénarios, estimer des débits, et réaliser des études stratégiques d'impacts environnementaux. En fin de compte, les planificateurs doivent reconnaître que le résultat idéal « gagnant-gagnant » est peu probable et qu'ils devront donc trouver la meilleure option «plus gagnant - moins perdant».

Les expériences en matière de rétablissement d'écosystèmes suite à des catastrophes naturelles ou des dommages dus aux hommes ont permis de formuler plusieurs principes clés à intégrer dans la planification d'une restauration après une catastrophe :

- Saisir l'occasion de mieux faire les choses

- Ne pas simplement planifier de recréer ce qui existait avant

- Choisir de travailler dans les zones les plus viables

- Toutes les régions affectées par une catastrophe n'ont pas besoin d'une réhabilitation active

- Concevoir un plan suffisamment flexible pour être adapté

- Présumer que le plan pourra changer en cours de réalisation

- Ne pas imaginer une stratégie " taille unique »

- Eviter que les actions entreprises ne causent de nouveaux dommages à l'environnement.

\section{L'INFLUENCE DES CHANGEMENTS CLIMATIQUES SUR}

\section{LA PLANIFICATION DE LA RESTAURATION}

Enfin, en préparant un programme de restauration, il sera vital d'inclure les impacts potentiels des changements climatiques futurs, aussi bien en termes d'adaptation que de mitigation. Les options devraient comprendre des efforts pour atténuer les émissions de gaz à effet de serre (GES), éviter les mesures qui risqueraient d'augmenter les émissions, et préparer des mesures qui aideront les communautés affectées à s'adapter aux changements climatiques prévus.

Étant donné les leçons tirées lors de la gestion de réponses apportées à d'autres catastrophes et ce que l'on sait aujourd'hui des facteurs aggravants comme les impacts des changements climatiques, toute mesure prise dans le cadre du plan de restauration devrait tenir compte des écosystèmes. Cela signifierait aussi de s'adapter à l'avenir en étudiant attentivement quelles espèces utiliser pour la reforestation et à quel endroit relocaliser les villages pour réduire leur vulnérabilité face aux risques futurs.

Une restauration complète exige des efforts gigantesques et coordonnés, et un investissement de ressources considérable. Mais c'est aussi l'occasion d'unir les gens et de créer un avenir meilleur, grâce à une gestion durable des services écosystémiques qui aide au développement, et de prendre des mesures pour atténuer les changements climatiques ou pour s'y adapter.

L'occurrence d'événements extrêmes et de catastrophes, spécialement sous l'influence des changements climatiques, est de plus en plus inquiétante pour l'environnement. Au cours de la prochaine décennie, la communauté de la conservation devra tirer les leçons des expériences d'adaptation aux changements climatiques basée sur l'écosystème, et les appliquer pour aider à réduire la vulnérabilité des gens face à ces événements extrêmes. Nous devons aussi encourager une meilleure gestion des services écosystémiques qui vont protéger les communautés de ces événements extrêmes et proposer des options de reconstruction et d'adaptation. 


\section{Santé humaine et biodiversité : 11. que peut faire la conservation ?}

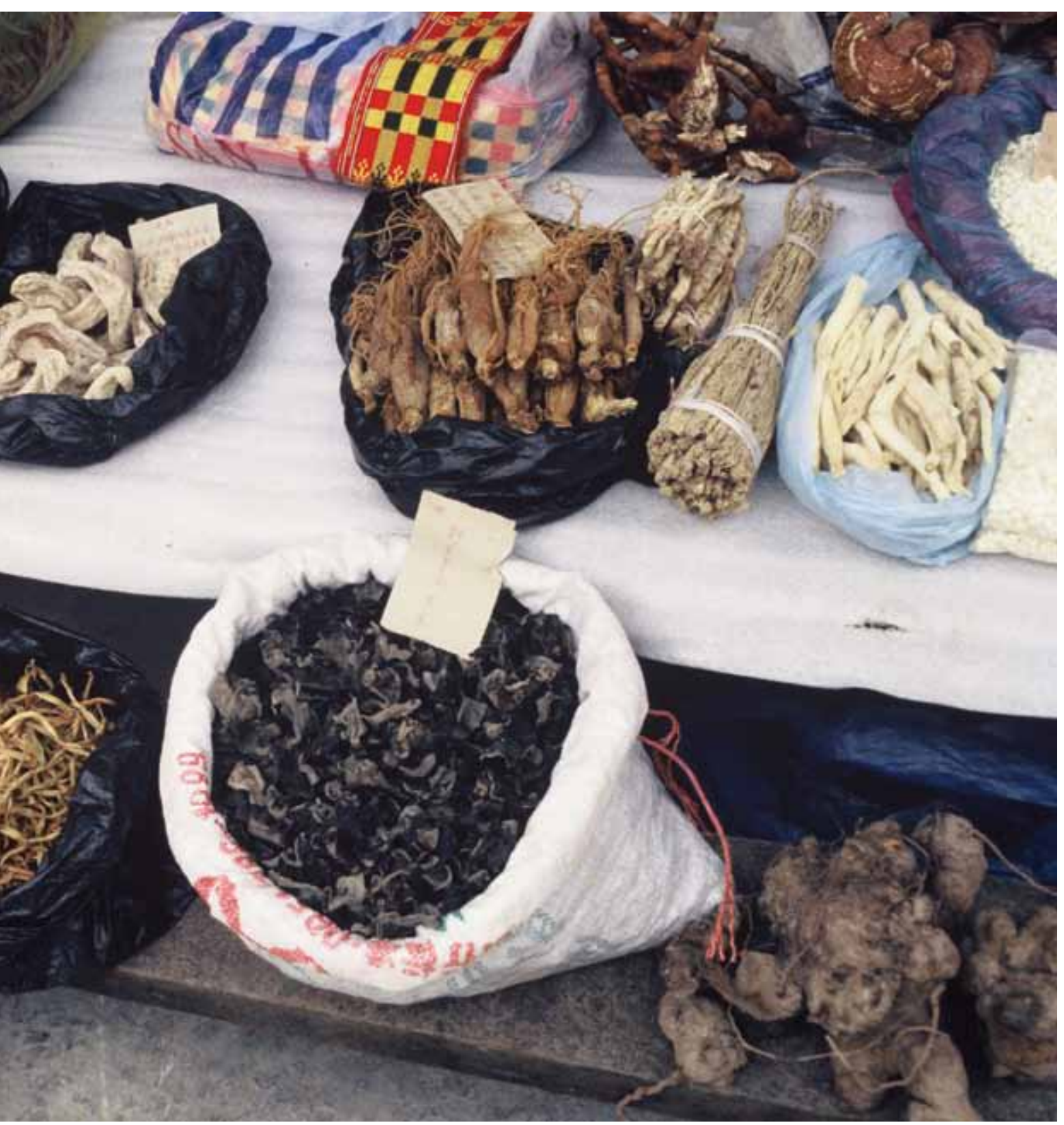


À un moment, au 20 ème siècle, on a cru que les maladies transmissibles étaient pratiquement vaincues, ou au moins sous contrôle. Mais aujourd'hui, la pandémie de SIDA s'étend, des agents infectieux toujours plus nombreux deviennent résistants aux antibiotiques, un nombre croissant de maladies endémiques explosent dans des endroits où elles étaient sous contrôle, les agents pathogènes se répandent et de nouvelles maladies voient le jour plus vite que la société ne peut y donner de réponse.

Alors que l'état sanitaire mondial devient alarmant, la relation entre santé et biodiversité fait l'objet d'une attention plus grande (Chivian et Bernstein, 2008). Quels sont les liens entre santé, changements climatiques, et biodiversité ? Pourrions-nous donner des réponses politiques plus efficaces en étudiant ces liens ? Les alliances entre scientifiques et décideurs politiques concernés par ces secteurs respectifs pourraientelles conduire à des mesures plus efficaces ?

La vie urbaine et les technologies modernes font que le public en général a moins conscience du fait que le maintien d'une bonne santé humaine dépend d'un environnement naturel sain. De plus, la recherche médicale actuelle tend à se focaliser sur les comportements à risque ou sur les organismes qui causent des maladies particulières. On accorde beaucoup trop peu d'attention aux facteurs influant sur la santé qui agissent au niveau des populations, comme la qualité de l'eau et des sols, les polluants qui modifient le système immunitaire, les produits chimiques organiques qui perturbent le système endocrinien et augmentent le risque de certains cancers, ou les changements climatiques qui influencent la dispersion des vecteurs de certaines maladies, comme les moustiques responsables de la malaria et de la dengue. Il faut aussi penser à ces facteurs dans des approches plus globales de la santé humaine.

Même si elle ressort de l'économie mondiale, de nombreuses personnes croient encore que la santé est d'abord une question personnelle, la prévention et les traitements étant centrés sur l'individu. Mais la santé est aussi une caractéristique des populations, et l'envisager dans une perspective plus holistique et plus large de la société peut mener dans une tout autre direction. Bien sûr, c'est l'individu qui contracte une maladie particulière, mais ce risque est largement influencé par le contexte sociologique et écologique où vit la population.

Tout confirme l'importance de la biodiversité dans la complexité des problèmes de santé, que ce soient les maladies infectieuses émergentes résultant de la destruction et de la fragmentation des forêts tropicales et d'autres écosystèmes 
(comme le virus Ebola), les liens sanitaires entre hommes et faune sauvage (comme le virus du Nil oriental), les nombreux produits pharmaceutiques connus et encore inconnus qui se trouvent dans la nature, la contribution des écosystèmes à la santé humaine, la reconnaissance croissante des disrupteurs endocriniens dans la santé humaine et animale ou l'effet des changements climatiques sur les écosystèmes.

Les composantes de la biodiversité peuvent se révéler des alliées ou des ennemies de notre santé. Les bactéries et les virus peuvent causer des maladies ; les grands carnivores, les créatures venimeuses et certaines plantes peuvent nous tuer; des conflits avec de petits herbivores comme certains rats ou des grands comme les éléphants peuvent saborder nos systèmes de production de nourriture, et par là même notre alimentation ; et certaines espèces non indigènes menacent notre santé.

Les bienfaits de la biodiversité sur notre santé, comme les médicaments extraits de plantes ou d'animaux et les services écosystémiques qui nous fournissent eau et air purs excèdent de loin les impacts négatifs. Si de nombreuses études se focalisent sur les bienfaits d'espèces animales et végétales sur notre santé, d'autres aspects de la biodiversité sont aussi importants pour la santé. La nature peut être aussi une source de santé mentale et physique, spécialement pour les jeunes (Louv, 2005). Pourtant certains de nos actes d'aujourd'hui menacent notre santé de demain et pour bien plus longtemps encore. La dégradation environnementale causée par la perte et la surexploitation d'habitats et les changements climatiques peut avoir des implications pour la santé et le bien-être humains.

\section{BIODIVERSITÉ : COMMENT LES ESPĖCES VÉGÉTALES ET ANIMALES CONTRIBUENT À LA SANTÉ}

Notre compréhension de la biodiversité médicinale est fondée sur les espèces. Le rôle des espèces dans le support de la santé humaine va des sources directes de produits pharmaceutiques à des modèles pour la recherche. D'autre part, la façon dont nous gérons la biodiversité (y compris nous-mêmes) peut avoir des effets sur la santé humaine.

\section{Une alliée pour la santé}

Quelque 50.000 - 70.000 espèces végétales servent en médecine, et 15.000 d'entre elles sont menacées (Schippman et al., 2006). La fabrication et la production de produits pharmaceutiques dérivés de plantes médicinales peuvent renforcer la santé humaine et préserver la biodiversité des plantes. Près de $80 \%$ de la population mondiale utilise des remèdes et des médicaments qui contiennent des agents végétaux actifs, extraits dans les pays mêmes où ils sont consommés. Le commerce mondial de plantes médicinales a augmenté de 85\% depuis 1991, encore que la grande majorité de ce commerce ne concerne qu'une douzaine de pays.

Les espèces médicinales font partie de la médecine traditionnelle et de la pharmacopée « occidentale ». Des problèmes tels que l'obésité sont maintenant traités au moyen de plantes médicinales venues d'Afrique, comme Hoodia $s p$. La plupart des connaissances traditionnelles autochtones ont une connexion directe avec le monde de la nature. De nombreuses pratiques traditionnelles se sont avérées avoir des effets positifs mesurables et elles peuvent mener à des découvertes de plantes ou d'animaux médicinaux.

Les connaissances traditionnelles (CT) en matière de santé sont souvent différentes chez les hommes et chez les femmes. Une étude ethnobotanique réalisée au Parc National de Jaú, au Brésil, a révélé que les sagesfemmes connaissaient certaines plantes et que les guérisseurs traditionnels hommes en connaissaient d'autres. Dans la communauté de Los Guatuzos, au Nicaragua, lorsque l'on 
demandait aux hommes quels types de plantes médicinales on trouvait dans leur parcelle ou en forêt, ils demandaient à leur épouse de répondre à cette question (Azofeifa, 2003, dans Rodriguez, Blanco et Azofeifa, 2004).

De nombreux animaux donnent aussi d'importants modèles de recherche en santé humaine, comme les implications pour l'ostéoporose du fait que des ours hibernent sans perdre de masse osseuse (Chivian et Bernstein, 2008). Les animaux ont aussi des physiologies uniques qui ouvrent des perspectives intéressantes qui pourraient améliorer la santé humaine, comme les grenouilles qui couvent leurs œufs dans leur estomac qui pourraient aider à comprendre les ulcères peptiques (encore que ces grenouilles sont peut-être éteintes maintenant).

De nombreux animaux fournissent aussi des médicaments, souvent à base de toxines utilisées pour l'agression (par exemple, les serpents venimeux et les cônes toxiques) ou la défense (les amphibiens).

\section{Un défi pour la santé}

Notre espèce abrite plus de microorganismes dans le corps ou en dehors que nous n'avons de cellules. De ce nombre surprenant, plus de 1400 peuvent être pathogènes pour l'homme, au moins dans certaines conditions. Ils comprennent au moins 217 virus et prions, 538 bactéries et rickettsies, 307 champignons, 66 protozoaires et 287 helminthes (Chivian et Bernstein, 2008). La plupart du temps, notre corps dépend de ces microorganismes pour son bon fonctionnement, mais cette abondance et cette diversité sont aussi autant de possibilités d'accès pour de nouvelles maladies. Si les hommes hébergent une tellement grande diversité d'organismes, c'est parce que nous sommes d'excellents hôtes pour une dispersion mondiale, que nous voyageons bien plus loin et que nous occupons beaucoup plus d'écosystèmes que toute autre espèce.
Comme ces microorganismes entrent de plus en plus en contact les uns avec les autres, par nos voyages ou par des changements des écosystèmes, de nouvelles maladies apparaissent. Par exemple, près de 190 espèces d'arbovirus et d'autres virus ont été identifiées en Amazonie brésilienne entre 1954 et 1998 (Vasconcelos et al., 2001). L'Amazonie brésilienne est très riche en arbovirus, ce qui reflète sa riche biodiversité générale. On sait très peu de choses de la plupart de ces virus. Les changements environnementaux qui entrainent la perte de la biodiversité - à savoir la déforestation, l'exploitation minière, la construction de barrages et d'autoroutes, la colonisation par les hommes et l'urbanisation sont les principaux changements que l'on associe à l'émergence, ou la réémergence d'arbovirus adaptés, et certains sont pathogènes pour les hommes. D'autres maladies sont aussi des effets secondaires de la perte de biodiversité.

Des relations écologiques modifiées peuvent augmenter les risques épidémiologiques à cause de l'introduction de nouveaux agents pathogènes chez de nouvelles populations. Par exemple, quand les hommes se dispersent vers des aires de nidification plus intense d'oiseaux sauvages, ils peuvent augmenter les risques d'échanges génétiques. Un mécanisme particulièrement inquiétant est l'échange génétique entre des virus infectant des hommes et des animaux sauvages ou domestiques, les deux virus prélevant des gènes l'un de l'autre, ce qui leur permet de produire une nouvelle enveloppe externe et de sortir du système immunitaire humain. C'est le principal mécanisme en cause dans les pandémies de grippe, qui impliquent souvent un virus humain et un autre porté par des canards, notamment des canards sauvages, et par d'autres espèces d'oiseaux.

Certains programmes de contrôle des maladies qui ciblent les microorganismes, même s'ils 
sont fructueux, minent souvent la santé s'ils perturbent des systèmes écologiques. Un exemple en est l'application de DDT, utilisé pour tuer les moustiques vecteurs de la malaria. Cela eut pour résultats inattendus de perturber les interactions entre insectes pollinisateurs, de réduire la reproduction de certaines espèces d'oiseaux et de faire baisser la production alimentaire. Les projets de développement agricole, conçus pour améliorer la santé grâce à une meilleure alimentation, peuvent aussi perturber les écosystèmes en modifiant le schéma des maladies. Pour favoriser durablement une bonne santé, il faut envisager de multiples échelles, de multiples perspectives et un degré non négligeable d'incertitude, et donc adopter une approche par écosystème des problèmes de santé.

L’Organisation mondiale de la santé (OMS) (2002) notait que les aléas environnementaux sont responsables d'environ $25 \%$ du poids total des maladies dans le monde, et jusqu'à 35\% en Afrique subsaharienne. L'amélioration des conditions environnementales pourrait sauver 13 millions de vies par an. Une meilleure gestion de l'environnement pourrait empêcher 40\% des décès dus à la malaria, $41 \%$ des décès dus à des infections des voies respiratoires et $94 \%$ des décès dus à des maladies diarrhéiques - trois des principales causes de mortalité infantile. Garantir la santé des écosystèmes peut aussi permettre de réduire la vulnérabilité des gens aux impacts des événements naturels extrêmes.

Les impacts sur la santé liés à la biodiversité peuvent se combiner à d'autres menaces. La mondialisation, par exemple, avec le nombre croissant de voyageurs et de biens qui traversent la planète, est en train d'étendre l'aire de dispersion de nombreux virus potentiellement dangereux pour les hommes. Les changements climatiques ont des impacts sur la biodiversité - ils changent la distribution et le schéma des chutes de pluies qui ont des effets secondaires sur la santé humaine, comme des changements de la distribution des vecteurs de maladies et aussi de l'approvisionnement en eau et en nourriture. Patz et al. (2004) notent le lien qui existe entre changement d'aménagement du territoire et émergence de maladies infectieuses, et insistent sur le besoin d'adopter une perspective plus large en matière d'aménagement du territoire en raison des relations complexes entre les écosystèmes d'un même paysage.

\section{OPTIMISER LE SUPPORT DE LA SANTÉ HUMAINE FONDÉ SUR LA BIODIVERSITÉ}

La gamme compète des services écosystémiques vient en aide à la santé humaine. Elle comprend les services d'approvisionnement, sous la forme de médicaments ou de nourriture, les services de support fournis par les sols pour une meilleure alimentation, les services de régulation, concernant la qualité de l'eau et de l'air, et les services culturels qui viennent soutenir la santé mentale. Les recherches ont montré que l'interaction avec la nature a un effet calmant sur les gens, que le temps passé dehors peut rétablir la santé, soulager le stress et donner un certain équilibre à la vie. Ces valeurs qui trouvent leur source dans le contact avec la nature sont universelles et elles ne se limitent pas aux pays « développés ». Lorsque l'on demande aux gens d'imaginer un endroit paisible, la grande majorité cite un endroit dans la nature, comme une plage, une forêt ou un lac.

Il est essentiel de reconnaître comment les changements du monde d'aujourd'hui peuvent affecter la biodiversité et la santé pour savoir d'où les menaces peuvent surgir et comment y répondre. Les environnementalistes peuvent apporter leur contribution en valorisant les plantes médicinales et en encourageant la recherche et l'utilisation durable des espèces indigènes qui sont intéressantes pour l'industrie de la santé, en reconnaissant l'impact de la nature 
dans tous les aspects de la santé et en se focalisant sur des politiques liées à la biodiversité.

La protection des plantes médicinales concerne de nombreuses parties prenantes. Ceux qui s'intéressent à la conservation de la nature se focalisent principalement sur la protection des habitats, la récolte durable dans la nature, les contrôles appropriés des commerces, etc. Ceux dont l'intérêt est plus social viseront la reconnaissance des savoirs traditionnels et des revenus réguliers suffisants pour permettre à ceux qui récoltent et aux agriculteurs d'avoir un revenu viable. Ceux qui s'intéressent plus au côté économique s'inquièteront de normes de qualité, de commerce prospère et d'une industrie de la transformation profitable donnant lieu à un commerce lucratif. L'OMS, avec l'UICN, le WWF et TRAFFIC, a rédigé des lignes directrices pour la conservation d'espèces d'intérêt médicinal (1993). De plus, la Norme internationale pour la collecte sauvage soutenable de plantes médicinales et aromatiques (ISSC-MAP) (Groupe CSE/UICN de spécialistes des plantes médicinales, 2007) a été créée par un partenariat industrie - communauté de la conservation - gouvernement. Elle se fonde sur les lignes directrices de 1993 ainsi que sur les Bonnes pratiques en matière d'agriculture et de collecte (GACP) de plantes médicinales (OMS, 2003). C'est un véhicule clé pour une future approche participative de l'utilisation et du commerce durables des plantes médicinales et aromatiques prélevées dans la nature.

Les liens entre santé humaine et animale sont aussi explorés par les conservationnistes. La Wildlife Conservation Society, l'UICN et des partenaires ont lancé un programme intitulé
Santé animale pour l'environnement et le développement (AHEAD) lors du Vème Congrès mondial des parcs, en 2003. Depuis lors, AHEAD a réuni différentes parties prenantes de toute l'Afrique australe pour examiner au niveau du paysage le réseau constitué par le triangle de la santé de la faune sauvage, celle de la faune domestique et la santé et les moyens de subsistance humains, appuyés par une bonne gouvernance environnementale.

Ils se sont particulièrement intéressés à l'Aire de Conservation transfrontalière (TFCA) du Great Limpopo où AHEAD favorise un dialogue et une planification multidisciplinaires sur la gestion des maladies de la faune sauvage et du bétail (dont des zoonoses - ces maladies transmissibles entre animaux et hommes) dans le paysage transfrontalier en développement. Dans les aires de conservation transfrontalières, les corridors ne servent pas seulement à connecter les animaux et les gens; ils constituent aussi des « ponts biologiques " pour les vecteurs et les agents pathogènes qu'ils véhiculent. Le besoin d'une approche holistique des activités de planification de l'aménagement du territoire à une si grande échelle ne pouvait pas être plus pressant. Dans les TFCA comme le Great Limpopo, les clôtures tombent déjà, ce qui donne à la faune sauvage et au bétail un accès à de nouveaux espaces et aussi entre eux pour la première fois depuis des décennies. C'est peut-être un moment important pour les revenus que rapportent la conservation et le tourisme nature (photographies, chasse, etc.), mais cela exige de jeter un regard plus attentif à certaines des implications. Quels effets ces aires transfrontalières pourraient-elles avoir sur la santé et la durabilité de la faune sauvage, 


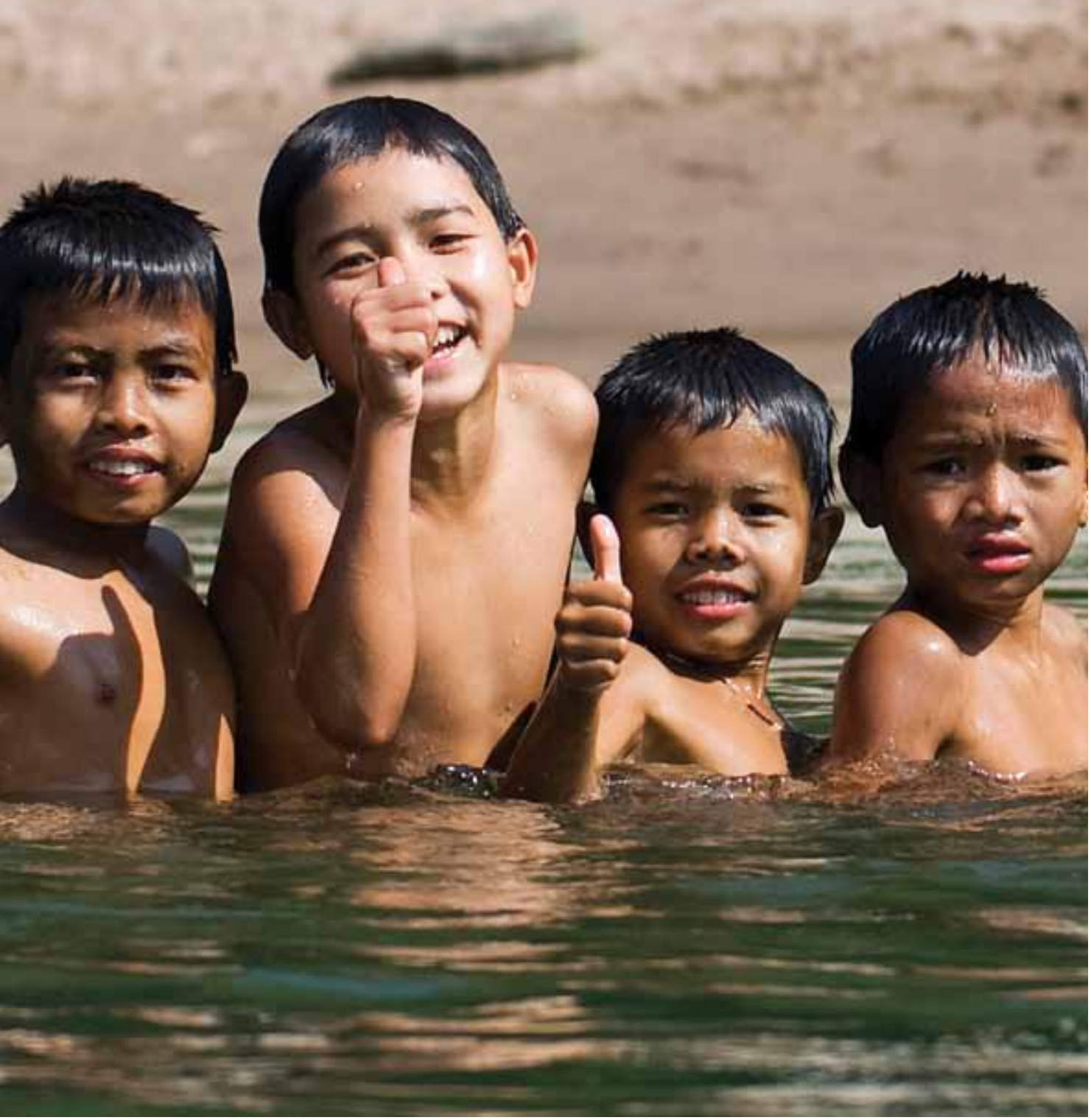


des animaux domestiques et des communautés humaines ? AHEAD, mécanisme rassembleur et facilitateur, travaille activement pour créer des environnements favorables qui permettront à des secteurs différents et souvent rivaux de s'asseoir littéralement à la même table et de trouver des moyens de relever ensemble ces défis et de s'occuper de la santé et des maladies non pas isolément mais dans un contexte socioéconomique et environnemental régional donné (Osofsky et al., 2005).

La croissance de la population humaine, la mondialisation et le commerce international, ajoutés aux changements climatiques, accélèrent la perte d'habitats, introduisent de nouvelles souches de maladies et changent la façon dont les systèmes naturels se régulent eux-mêmes. Une plus grande diversité d'espèces remplissant des fonctions similaires dans un écosystème pourra augmenter la probabilité de préserver les processus écosystémiques en cas de changement environnemental. Du point de vue de la santé humaine, plus grande est la diversité d'un écosystème, plus il est probable que les services écosystémiques dont dépend notre santé continueront à être produits.

Observer la biodiversité par la lunette de la santé humaine permet d'avoir une autre perspective sur la politique et les pratiques de la conservation de la biodiversité, dans le but de préserver la santé humaine. De la même façon, on peut dire que conserver la biodiversité pour le bien de la santé humaine peut amener un plus grand public à de meilleures pratiques de conservation. Le fait de montrer les liens qui existent entre la conservation et la santé humaine met la conservation au centre des préoccupations humanitaires.

Si l'on pense à l'importance évidente de toutes les composantes de la biodiversité pour la santé humaine, la pratique d'une conservation de la biodiversité qui appuie le maintien ou l'amélioration de la santé humaine va exiger un meilleur partage des connaissances, l'extension des partenariats, la gestion des ressources de la biodiversité à l'échelle du paysage et la mise en pratique de politiques et de lignes directrices, telles les ISSC-MAP, en faveur de la conservation de la biodiversité médicinale. 
12. Développer une économie « verte»

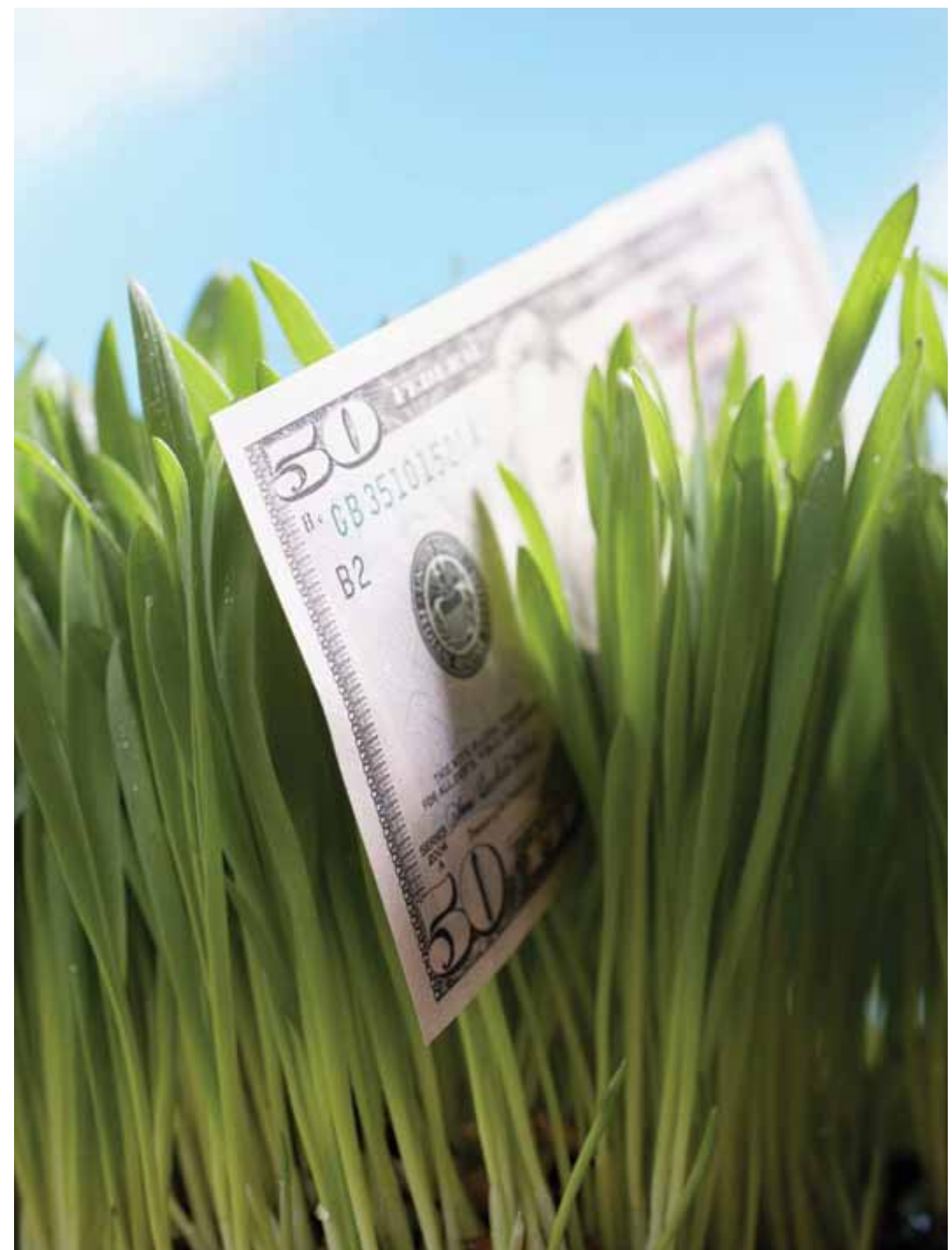


La crise financière de 2008 et les renflouements financiers et les mesures d'austérité qui y ont répondu pourraient avoir des implications encourageantes pour les conservationnistes : avec un système prêt pour le changement, il serait possible de trouver de la place pour une économie verte. Mais les inquiétudes suscitées par le système économique qui s'effondre détournent l'attention d'un autre système qui est au bord du gouffre, l'écosystème mondial.

Les changements climatiques, la pêche qui diminue, l'accès de plus en plus restreint à l'eau potable, des écosystèmes dégradés au-delà de toute possibilité de se restaurer, la litanie peut continuer comme le révèle l'Evaluation des écosystèmes pour le millénaire (EM). Un modèle de croissance semblable à celui qui a conduit à la crise financière est en train de nous pousser vers une crise écologique aux dimensions dévastatrices. Un modèle de croissance qui défie toute limite et externalise ses coûts est difficilement durable. Mère Nature, contrairement aux gouvernements, ne fait pas de « renflouement».

Le système opérationnel actuel de l'économie monétaire est fondé sur une production non durable d'énergie et de biens matériels et sur la consommation des biens produits et des services qui les accompagnent. Les gens sont heureux de consommer et il est certain que beaucoup en veulent encore. Pourtant, les impacts d'une consommation non soutenable sur l'environnement sont de mieux en mieux connus et des consommateurs commencent à réagir. Le National Geographic, en collaboration avec Globescan, a mis au point un indicateur « Greendex » pour mesurer le choix des consommateurs et l'environnement. Leur rapport 2009 donne des informations sur 17 pays, d'où il ressort que l'Inde et le Brésil sont les consommateurs les plus sensibles au point de vue de l'environnement tandis que les Canadiens et les Américains se trouvent au bas du tableau (Figure 12.1).

Comment l'acte de consommer pourrait-il être rendu neutre par rapport à l'environnement à l'échelle mondiale et pas seulement dans les pays qui reçoivent la plus haute cotation du Greendex ? C'est le défi majeur d'une économie verte. Au niveau individuel, vivre durablement est une question de gestion de notre consommation personnelle, pour qu'elle n'exerce pas une pression excessive sur la Terre. À l'autre bout de l'échelle, la durabilité d'une entreprise consiste à rendre plus à la Terre qu'elle ne lui prend. L'économie verte reconnaît que l'environnement, l'économie et la société sont tous des éléments de la même feuille de calcul. La santé de l'économie, basée sur la consommation et la production, est en équilibre avec la santé de l'environnement. 
GREENDEX: CLASSEMENT GÉNÉRAL DES CONSOMMATEURS DANS CHAQUE PAYS, 2008-2009
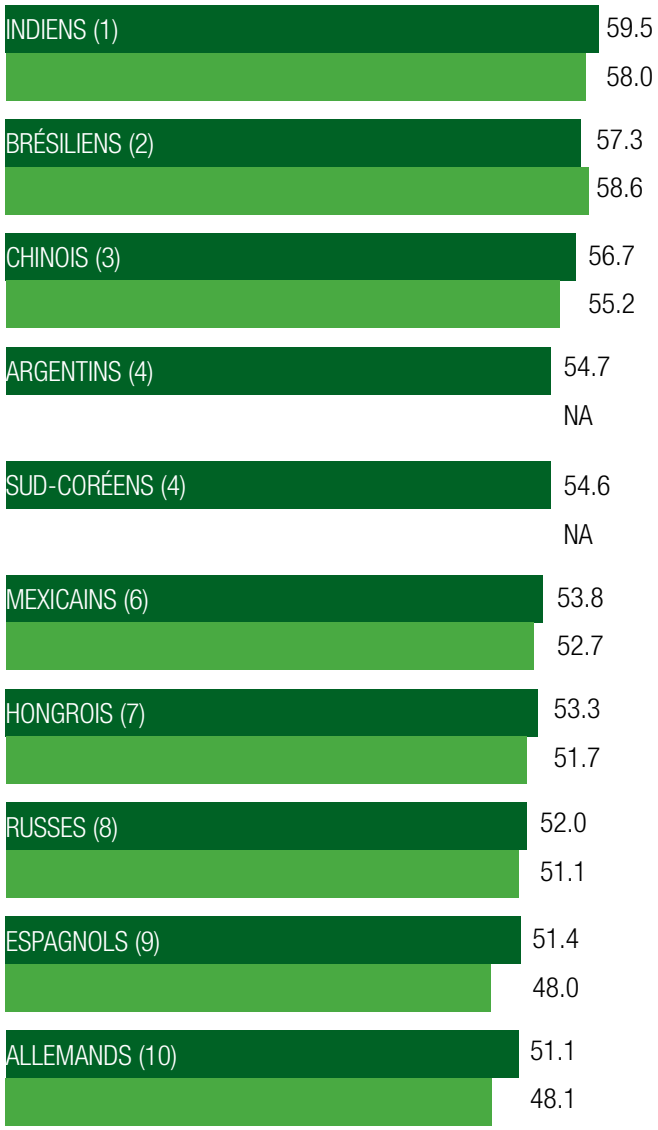

SUÉDOIS (10)

NA
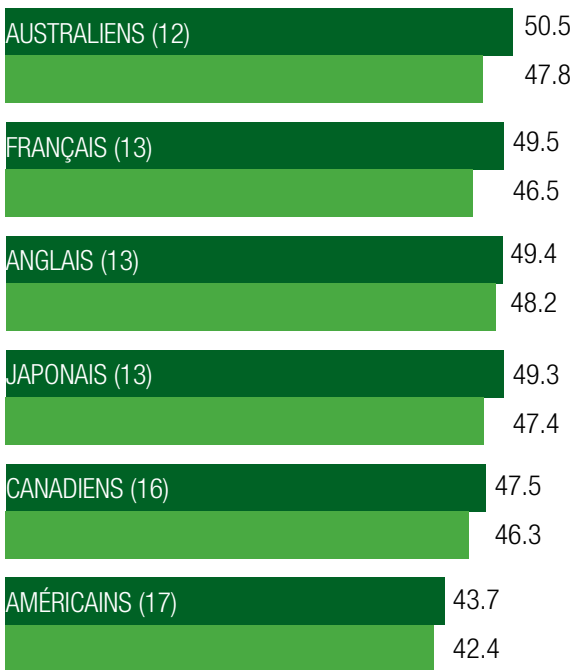

Figure 12.1 Greendex 2009 (National Geographic)

\section{MESURER LA DURABILITÉ}

Une des clés pour une terre plus équilibrée et plus saine est de se dissocier de l'idée que l'utilisation des ressources naturelles est un moteur de croissance économique. En intégrant l'environnement et les services écosystémiques dans le calcul de l'économie mondiale, on peut utiliser des instruments économiques pour soutenir l'environnement, sa protection et sa conservation. Mais la façon dont nous mesurons le progrès actuellement, par le Produit intérieur brut (PIB), l'Indice de développement humain (IDH) et d'autres indices semblables, ne reflète pas correctement la contribution de l'environnement.

L'impact environnemental de la surconsommation dans les pays développés est amplifié du fait que les prix des biens et des services naturels ne reflètent pas correctement le coût véritable pour les écosystèmes et l'environnement en général. Les personnes fortunées endommagent l'environnement par leurs habitudes de consommation, entraînant des forces du marché qui épuisent les ressources naturelles locales et dans le monde en développement, où les normes environnementales n'existent pas ou sont peu appliquées.

\section{OUTILS DE TRANSITION VERS UNE ÉCONOMIE VERTE}

\section{Valorisation de l'environnement}

Les études économiques cherchent de plus en plus à comprendre la valeur de l'environnement pour notre économie, et le coût de l'inaction ou du fait de ne pas le préserver. Ten Brink (2008) a estimé que si nous ne stoppons pas aujourd'hui la perte de biodiversité, les coûts cumulatifs pour été recalibrés d'une façon qui ne modifie pas la signification des résultats 
le bien-être humain, à cause de la perte des seuls produits et services forestiers, pourraient atteindre 14 trillions d'euros (7\% du PIB mondial) en 2050.

L'Économie des écosystèmes et de la biodiversité (TEEB, Chapitre 4) vise à une meilleure appréhension des coûts réels de la consommation, ainsi que des problèmes d'externalité dans les chaînes d'approvisionnement et les systèmes de production modernes (TEEB, 2008). Les chaines d'approvisionnement sont l'ensemble des producteurs, transformateurs et distributeurs qui achètent et vendent les uns aux autres pour fournir des produits finis aux consommateurs. Les gestionnaires de chaînes d'approvisionnement qui recherchent des bons rendements parviennent parfois à faire des économies en refusant de payer le prix total d'une production durable. On en prendra pour exemple l'« économie » réalisée en ne traitant pas l'eau polluée, ou en ne compensant pas les puits de carbone perdus lors du dénuement de terrains. Ce sont des coûts réels qui doivent se refléter dans les chaînes d'approvisionnement et, en fin de course, chez les acheteurs, pour que les consommateurs comprennent la valeur des services écosystémiques qui sont intervenus dans la production du produit qu'ils ont acheté.

\section{INCITANTS - LE BON ET LE MAUVAIS}

Les mécanismes du marché utilisés pour favoriser la conservation sont extrêmement variés et ils vont du simple schéma de tarification de l'eau à des fonds spéculatifs (bedge funds) sophistiqués. Idéalement, tout mécanisme financier de ce genre, plutôt que de fournir un financement unique, devrait fonctionner comme un incitant continu de la conservation. En tenant compte, par ces mécanismes, du rôle à long terme des écosystèmes, on intègre la durabilité dans les décisions économiques. Évidemment, ces mécanismes ne fonctionnent que s'il y a des normes crédibles, et des systèmes de vérification et de mise en application.

\section{Compensations}

Une compensation est une mesure prise pour contrer ou pour compenser l'impact d'autres actions. Pour les changements climatiques, le programme de compensation du carbone le mieux connu est le Protocole de Kyoto (et en particulier le Mécanisme pour le développement propre - MDP) qui fut inventé pour permettre aux pays qui avaient pris des engagements au sujet de leurs émissions dans le cadre du Protocole d'opérer certaines des réductions d'émissions exigées dans des pays en développement. Le MDP fut conçu pour aider à respecter les engagements intergouvernementaux, dans un cadre juridique contraignant, mais il existe aussi un marché volontaire du carbone qui, en 2008, a presque doublé tant au niveau du volume de carbone vendu que de sa valeur, par rapport à 2007, avec un montant total de 705 millions de dollars en 2008 (Hamilton et al., 2009). Des compensations ont aussi été appliquées dans certains cas à des espèces et à des habitats. Ces dernières applications sont relativement récentes mais le Business and Biodiversity Offsets Programme $(\mathrm{BBOP})$ cherche à définir des principes et des méthodologies pour soutenir les bonnes pratiques en matière de compensations volontaires de la biodiversité (BBOP, 2009).

\section{Paiements pour les services écosystémiques (PES)}

Les paiements pour les services fournis par les écosystèmes (PES) sont mentionnés fréquemment dans ce volume car ils sont un excellent moyen de refléter la valeur des bénéfices que les gens retirent de la nature (Wunder, 2005). Un service écosystémique pour lequel les plans de PES existent dans plusieurs pays, spécialement en Amérique latine, est le paiement pour la protection des bassins de rivière. Comme les consommateurs sont de plus en plus conscients des nombreux services fournis par les bassins 
de rivière (qualité et quantité de l'eau pour les industries et les besoins domestiques et récréatifs), ils sont plus motivés par leur conservation (Smith et al., 2006). Ces paiements peuvent être des paiements d'utilisateurs privés à des agences gouvernementales et à des organisations non gouvernementales (ONG) de conservation aussi bien que des paiements directs par le gouvernement central à des propriétaires privés. L'expérience montre que le paiement pour la protection des bassins est surtout indiqué lorsque :

- Il est trop coûteux (et inutile) d'acheter directement la ressource ;

- Les paiements sont moins chers que les solutions techniques alternatives (ex. infrastructures);

- La fourniture du service souhaité est vérifiable et mesurable ;

- Les coûts de transactions ne sont pas prohibitifs ; et

- Quelqu'un veut bien payer le prix (Kousky, 2005).

Le développement et la mise en route effectifs de marchés pour les services écosystémiques sont limités par plusieurs facteurs dont des organismes de marché trop faibles et surtout des droits de propriété mal définis, une reconnaissance inadéquate de la responsabilité en cas de dommages environnementaux, une absence de toute culture de la récompense pour des contributions positives au bon état de l'environnement, et des capacités médiocres en matière de réglementations (Bishop et al., 2009).

\section{Certification}

Les programmes et les normes en matière de biodiversité sont de plus en plus considérés comme des outils importants pour améliorer la performance de l'industrie dans le domaine de la biodiversité. Les programmes de certification supposent que les consommateurs vont préférer acheter ou même payer plus pour des biens et des services certifiés. La certification tient déjà une place importante dans plusieurs secteurs comme l'agriculture, la foresterie, le tourisme et les services financiers (Bishop et al., 2009). La valeur des produits certifiés est importante et elle s'accroît, comme la valeur du marché mondial des produits biologiques qui était de 23 milliards de dollars en 2002 (Willer et Minou-Yussefi, 2006). De même, le volume de la production certifiée s'accroît : par exemple, la superficie des forêts certifiées est passée de 5,8 millions d'hectares en 1998 (www.earthtrends.org) à plus de 300 millions d'hectares dans le monde entier à la mi-2008, la plupart se trouvant dans la région de l'UNECE, grâce à l'encouragement des systèmes de construction verte et des politiques publiques de passation de marché (UNECE et FAO, 2008).

\section{Subsides et incitants fiscaux}

Les incitants économiques de la conservation de la biodiversité servent depuis des décennies (McNeely, 1988) mais cette pratique est restée relativement modeste et il faut l'encourager de façon significative (comme le demande l'Article II de la Convention sur la diversité biologique - CDB) (CDB, 2004a). Dans certains pays, les incitants destinés à encourager la conservation des ressources comprennent des subsides et des incitants fiscaux, par exemple, sous forme de réductions fiscales pour des contributions caritatives. Ce mécanisme fut une motivation pour des cessions de terres aux États-Unis et en Europe, ce qui a permis de protéger des millions d'hectares (The Trust for Public Land, 2009 ; Bräuer et al., 2006).

Pourtant, il est possible que des incitants comme des subsides aient des impacts négatifs comme le montre la situation actuelle de la pêche mondiale. En 2000, on estime que 26 milliards de USD de subsides ont été payés au secteur de la pêche, dont 16 milliards étaient destinés à augmenter la capacité de pêche dans un monde où la majorité des stocks étaient déjà surexploités (Chapitre 17) (Sumaila et Pauly, 2006). 
FAIRE DE L'INVESTISSEMENT DANS LA GESTION DES RESSOURCES NATURELLES UN FACTEUR

DE CROISSANCE

Les ressources naturelles sont longtemps restées la base de la croissance économique.

Cette croissance mal gérée ou non contrôlée a causé de la pauvreté, des conflits et de la dégradation environnementale. Historiquement, le colonialisme était notamment attisé par la quête de ressources naturelles, en grande partie au bénéfice des pouvoirs coloniaux. Des exemples plus récents incluent le cuivre, le coltan et le cobalt produits en République Démocratique du Congo, les mines de diamants de la Sierra Leone et le pétrole du Nigeria.

La dégradation des ressources naturelles trouve sa source en grande partie dans une mauvaise gouvernance. Même des pays où la gouvernance est solide importent des ressources pour les transformer ou pour produire de l'énergie, un signe que la production n'y est pas durable et une justification indéniable du commerce mondial. La gestion durable des ressources naturelles au niveau local et mondial aurait le pouvoir de soutenir à long terme une croissance économique en faveur des plus pauvres et donc d'atteindre de plus vastes objectifs du développement.

L'utilisation des ressources naturelles peut contribuer à la réduction de la pauvreté et à la santé et au bien-être des populations. Il est essentiel de préserver un capital naturel pour garder un capital humain. L'OCDE (2008) en appelle à ce « capital naturel critique »- le seuil de capital naturel nécessaire pour qu'un autre capital, tel le capital humain, puisse exister. Les agriculteurs de subsistance, qui connaissent bien les processus agricoles locaux en sont un exemple. Si la fertilité du sol disparait, ils ne seront plus capables de produire et ils perdront leurs compétences humaines en même temps que le capital naturel du sol. Pour garantir que les ressources naturelles sont un facteur de croissance, qu'elles la portent et qu'elles contribuent à sortir les gens de la pauvreté, les pays développés cherchent des utilisations efficaces, équitables et durables de leurs ressources naturelles (Encadré 12.1).

Encadré 12.1 Des diamants pour le développement : le cas du Botswana

Le Botswana utilise sa richesse en ressources naturelles (diamants) pour réduire la pauvreté, grâce à la création d'un Fonds de stabilisation des revenus et d'un Fonds de service de la dette publique. Les diamants ne sont pas une ressource naturelle renouvelable, mais le capital humain, oui. En développant son industrie de transformation des diamants, en encourageant la diversification économique et en canalisant les revenus fiscaux du secteur des minerais vers ceux de l'éducation et de la santé, le Botswana a pu réinvestir les bénéfices de l'industrie diamantaire dans le relèvement du pays via le développement des institutions villageoises, la responsabilisation locale, l'identité et la culture villageoises et une dépendance moindre par rapport au soutien du gouvernement. Tout cela a permis de soulager la pression exercée sur l'environnement puisque les gens n'étaient plus tentés d'exploiter les ressources naturelles pour survivre.

Source: PEP, 2005

\section{UNE AUTRE OPTION : DÉVELOPPER UN NOUVEAU SYSTĖME ÉCONOMIQUE}

Les instruments économiques détaillés ci-dessus veulent intégrer les coûts environnementaux dans des politiques macroéconomiques. Si beaucoup applaudissent ce pas vers la réunion des systèmes naturel et monétaire, d'autres s'inquiètent parce que l'économie actuelle se fonde sur le modèle de la croissance perpétuelle et que, quelle que soit la façon dont nous amenderons le modèle, nous allons inévitablement continuer sur une 
voie insoutenable (p.ex. Speth, 2008). Le modèle d'aujourd'hui va bientôt atteindre le point où l'économie va dépasser la capacité qu'a la terre de la soutenir. Nous vivons au-dessus de notre capacité de charge (Encadré 12.2).

De nombreuses personnes (et pourtant pas obligatoirement une majorité d'économistes)

Encadré 12.2 Au-delà de la capacité de charge

En novembre 2005, l'Agence européenne pour l'environnement (AEE) a livré son rapport " L'Environnement en Europe, état et perspectives 2005 ». Il conclut qu'il faut 2,1 fois la capacité biologique de l'Europe pour soutenir l'Europe. Avec une population qui équivaut à $7 \%$ du total mondial, sa demande sur la capacité écologique mondiale est d'environ 20\% de la productivité mondiale. Cette capacité biologique pour l'Europe provient du reste du monde. Quel serait le prix correct à payer pour cette demande de surplus et comment pourrait-il être payé ? Source: AEE, 2005 croient que le monde doit investir dans un nouveau système, un qui ne donnera pas d'incitants pervers pour une croissance non durable. Le monde a besoin d'un système qui modifie les habitudes de consommation des gens, qui investit dans des infrastructures vertes (ce qui signifie aussi bien des investissements dans l'environnement lui-même en tant qu'infrastructure fondamentale que dans des infrastructures physiques construites de manière respectueuse de l'environnement) et qui pense stratégiquement à la façon dont nous vivons sur cette fragile planète. Les systèmes mondiaux qui s'effondrent montrent douloureusement qu'il est temps de changer fondamentalement. Les modèles proposés suggèrent une " économie circulaire » comme elle est exprimée clairement en Chine, où les objectifs économiques et environnementaux sont poursuivis en tandem et où « les déchets d'une unité de production sont les intrants d'une autre " (Pinter, 2006). McDonough et Braungart adoptent une philosophie similaire dans Cradle to Cradle (2002). Une autre approche vise une réforme

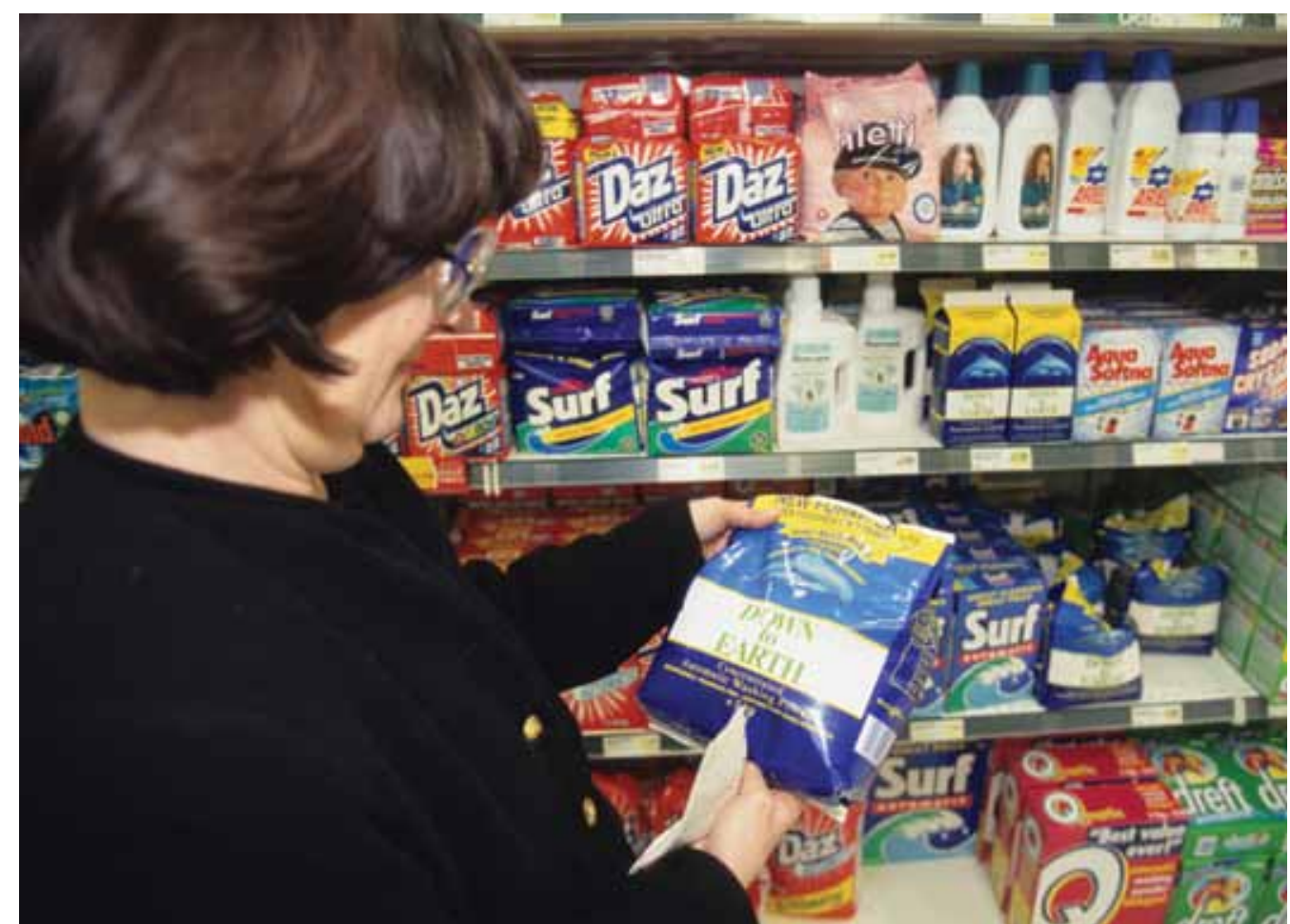


conceptuelle vers une économie durable qui encourage le développement plutôt que la croissance, qui intègre complètement les valeurs de la nature dans le système et qui applique une approche préventive de la politique économique publique (Worldwatch Institute, 2008).

Une autre option pour une économie verte est de retourner plus près de l'origine de la plupart des ses intrants. Le mouvement « acheter local » en est un indicateur. Si ses partisans reconnaissent qu'un marché mondial est indispensable, ils rejettent l'idée de dépendre totalement de l'économie mondiale et soutiennent le développement d'une plus grande autosuffisance locale pour tout, de la nourriture à l'énergie. Ceci impliquerait une plus grande collaboration au sein des communautés, une adaptation millénaire qui est négligée depuis les temps modernes (McKibben, 2007).

Quel que soit le modèle choisi, les choix personnels en matière de consommation et les valeurs de la nature seront des activateurs importants en faveur d'un système économique plus durable. Le soutien d'instruments économiques comme les compensations carbone peuvent se faire au niveau individuel chaque fois qu'une personne voyage, et l'achat de produits certifiés durables est un moyen de garantir que les ressources naturelles consommées sont gérées efficacement.

Une « économie verte » est une condition essentielle pour que l'UICN remplisse sa mission. Si la forme de cette nouvelle économie évolue rapidement grâce à des activités comme la TEEB, l'Initiative pour une économie verte du Programme des Nations Unies pour l'environnement (PNUE), le paiement des services écosystémiques et diverses initiatives nationales, les membres de l'UICN s'appliquent activement à vérifier que la biodiversité et des services écosystémiques y seront bien représentés.

\section{L'utilisation des ressources naturelles}

\section{peut contribuer à la réduction de la pauvreté et à}




\section{Technologie et conservation}

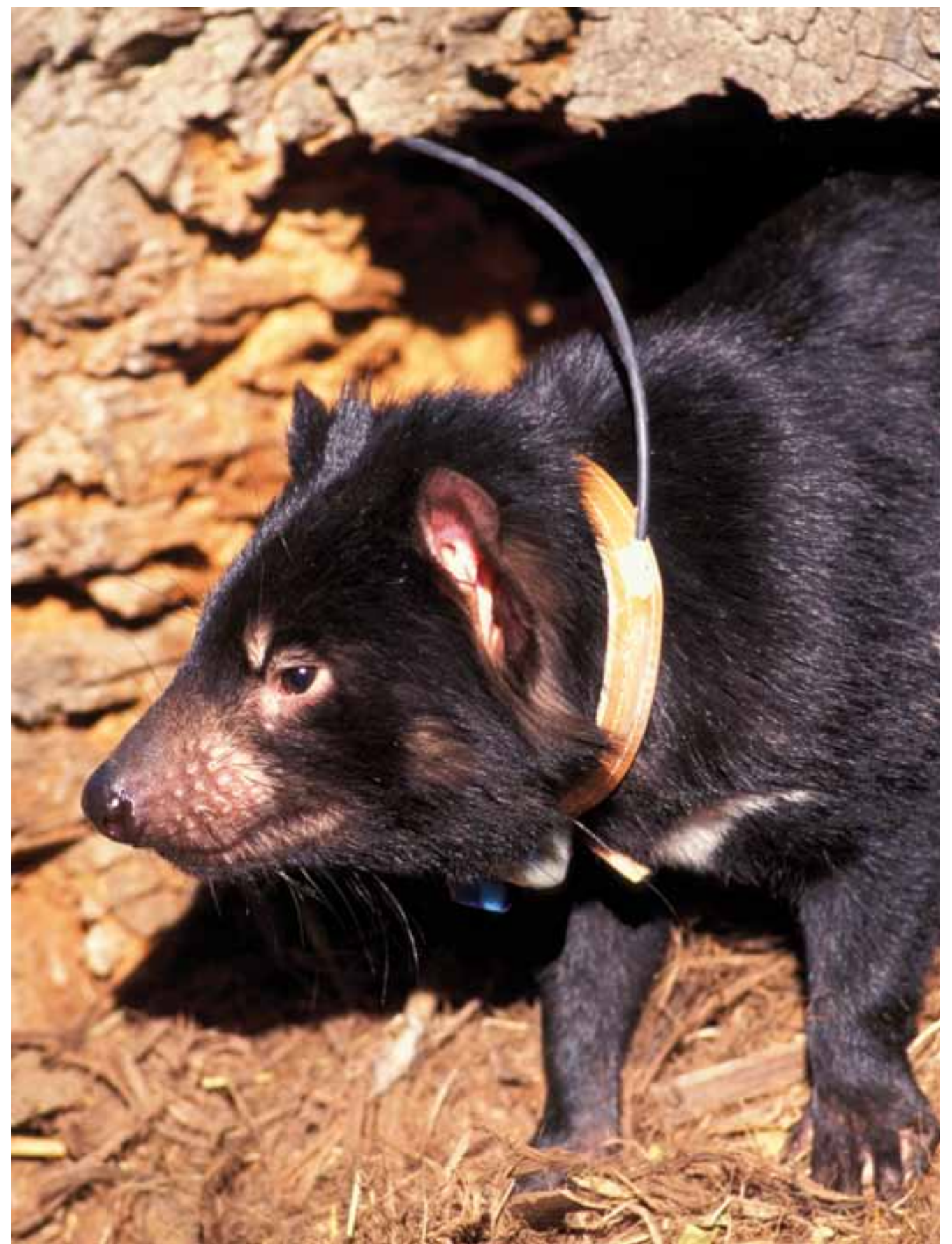


En octobre 1992, Julian Simon et Norman Myers ont eu un débat historique à l'Université de Columbia sur " la Rareté et l'abondance " (Myers et Simon, 1992). Alors que Myers, identifié alors par certains comme un " prophète du malheur ", arguait que tous les indicateurs environnementaux allaient dans la mauvaise direction et qu'en fin de compte, les hommes en paieraient le prix, Simon se félicitait de la croissance de la population humaine et affirmait que plus de gens signifiait plus d'intelligences pour développer des solutions technologiques pour les défis à venir. Cette discussion sur le pouvoir de la technologie qui pourrait vaincre les impacts humains sur notre monde est toujours en cours (Ehrlich et Ehrlich, 2008) mais les preuves s'accumulent pour montrer que Myers pouvait avoir raison déjà en 1992. Cela vaut quand même la peine de se demander si la technologie peut nous aider à éviter les pires implications du pronostic malthusien, une humanité qui excède la capacité de charge de la terre.

De par le monde, au cours des millénaires, les communautés ont développé leurs propres technologies. Les technologies traditionnelles ont cédé la place à des formes plus modernes, mais elles pourraient encore avoir beaucoup à nous offrir (Klee, 1980 ; Gadgil et Berkes, 1991). Nombre de ces technologies traditionnelles sont basées sur le bio-mimétisme et elles peuvent être améliorées par quelques éléments modernes. La Commission UICN des politiques environnementales, économiques et sociales (CEESP) a largement favorisé ces approches. Elles entrent de plus en plus dans le courant de pensée sur le développement et offrent un potentiel considérable dans le domaine des économies vertes.
Beaucoup de technologies modernes ont contribué à l'exploitation plus globale des ressources naturelles et n'ont pas anticipé les effets collatéraux qui ont causé certains des défis environnementaux les plus insolubles. Cependant, comme le dirait Simon, les nouvelles technologies sont aussi la base de certaines des solutions.

\section{QUELLES TECHNOLOGIES ET QUEL IMPACT ?}

Au point de vue de l'environnement, parmi les technologies clés qui ont à la fois aidé et entravé la conservation de l'environnement, on peut citer la technologie de gestion de l'information (TI), la biotechnologie et le géo-engineering et la technologie énergétique (Chapitre 8). 


\section{Technologie de l'information}

À l'époque du Sommet de la Terre de Rio de Janeiro, en 1992, personne n'avait de téléphone portable, Internet n'était pas encore opérationnel et les ordinateurs portables étaient considérés tout au plus comme des ordinateurs de bureau transportables. En un peu moins de 15 ans, la TI a fait des progrès remarquables qui entraînent aussi bien des coûts que des avantages pour la biodiversité.

Les coûts des progrès de la TI peuvent se calculer d'une part par les impacts d'un plus grand accès à l'information et d'autre part par ceux du développement et de la fourniture d'une technologie pour assurer cet accès. Un accès plus facile aux informations

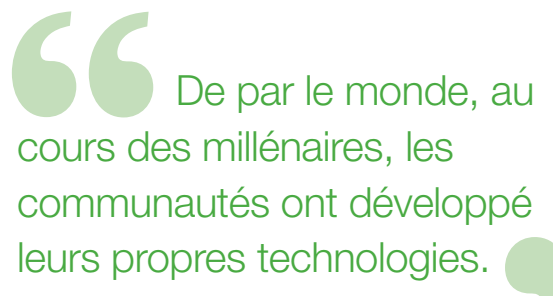
fait que ceux qui cherchent à exploiter la nature peuvent identifier où se trouvent les ressources intéressantes et les marchés potentiels. Les matières premières nécessaires aux ordinateurs et aux téléphones portables grâce auxquels nous récoltons et partageons nos connaissances peuvent avoir des effets négatifs sur l'environnement, tout comme la façon dont nous nous en débarrassons lorsqu'un nouveau modèle arrive sur le marché. L'exploration et l'exploitation de matières premières comme le coltan ont déjà des effets dévastateurs sur la biodiversité dans des endroits comme la République Démocratique du Congo. La fabrication et l'utilisation des ordinateurs et des téléphones portables consomment de l'énergie et de l'eau et ont donc un impact sur le climat.

L'utilisation des produits de la TI consomme d'énormes quantités d'énergie, que ce soit l'électricité pour faire fonctionner les PC ou les besoins des parcs de serveurs et autres infrastructures de la TI qui font fonctionner
Internet. La consommation annuelle des ordinateurs varie de 52 à $482 \mathrm{kWh}$, et celle des écrans va de 22 à $754 \mathrm{kWh}$ (Bray, 2006); les différences dépendent des spécifications et de l'âge des ordinateurs et des écrans testés. Par comparaison, disons que la consommation annuelle moyenne des réfrigérateurs aux États-Unis était de $1239 \mathrm{kWh}$ (http:// www.eia.doe.gov/emeu/reps/enduse/er01_ us.html\# Electricity).

Enfin, lorsque des ordinateurs obsolètes sont mis au rancart, le plomb, le mercure et les autres substances toxiques qui ont été utilisées pour leur fabrication peuvent causer de graves problèmes de pollution. L'importance de ces déchets est immense. En 2005, les équipements électriques usagés représentaient quelque deux millions de tonnes de déchets, dont la plupart étaient jetés dans des décharges. Rien qu'au Royaume-Uni, on jette 1700 téléphones portables par heure, 15 millions par an. Les métaux lourds et autres polluants comme le mercure, le plomb, le cadmium et les retardateurs de flamme bromés sont abandonnés et polluent les sols. Une grande partie du matériel électronique jeté par les pays industrialisés part vers des pays pauvres d'Afrique ou d'Asie dont les politiques environnementales sont inefficaces. Par contre, le recyclage des téléphones mobiles réduit les émissions de gaz à effet de serre (GES), permet de ne pas jeter en décharges et dans les incinérateurs des matières de valeur, et préserve les ressources naturelles. Le recyclage d'un million de téléphones portables a le même effet sur la réduction des émissions de GES que si l'on retirait 1368 voitures de la circulation pendant un an.

Si les technologies de la communication et de l'information (TCI) ne sont pas spécialement 
favorables à l'environnement, elles sont de plus en plus sollicitées pour améliorer la gestion des services écosystémiques et de la biodiversité. Par exemple :

- La consolidation des informations comme la Base de données mondiale sur les aires protégées (www. wdpa.org); source libre d'accès et téléchargeable d'informations sur les aires protégées du monde entier, elle est utilisée pour l'analyse écologique des lacunes, l'évaluation des impacts sur l'environnement, les prises de décisions dans le secteur privé et la création de nouveaux produits de données.

- Les synthèses d'informations, comme les guides de terrain électroniques sur les plantes ou les animaux, permettent aux scientifiques et aux touristes d'identifier les espèces qu'ils rencontrent dans la nature.

- La télédétection et le pistage d'espèces discrètes comme le léopard des neiges et le tigre permettent aux scientifiques de faire des recensements et même de photographier des espèces récemment découvertes comme le saola.

- La recherche dynamique des changements environnementaux aide à apporter une réponse aux feux de forêt ou aux changements climatiques.

- Les appareils portables, particulièrement les téléphones, permettent aux agriculteurs d'améliorer considérablement leur productivité et leurs profits, parce qu'ils ont un accès direct et plus précis au prix payé pour leur récolte, aux prévisions du temps et à de meilleurs régimes d'irrigation. En supprimant les intermédiaires, ces TCI peuvent aider les agriculteurs à se sortir de la pauvreté.

- Laccès de tous aux informations sur l'environnement, par exemple via Google Earth ou les nombreux sites qui ont des webcams braquées sur des animaux sauvages, a accru la sensibilisation et l'attention pour le monde naturel et pour les changements qui s'y passent.

- La TI a entraîné de nouvelles formes de démocratie en gestion des ressources, parce que les ruraux l'utilisent pour avoir un meilleur contrôle de leurs ressources naturelles.

- La détection à distance, qui passe souvent par des satellites pour récolter des informations géographiques temporelles sur la terre, est devenue une technologie majeure de la gestion environnementale, que l'on utilise dans de nombreux contextes.

Les progrès des TI se manifestent aussi dans la taille réduite des instruments. De nombreuses espèces élusives peuvent désormais être étudiées par radio-tracking, et l'on pose maintenant de minuscules émetteurs sur des papillons, ce qui montre le degré de miniaturisation auquel on est arrivé. On a attaché des caméras miniatures sur le corbeau de Nouvelle-Calédonie, en danger critique d'extinction, qui ont permis pour la première fois aux scientifiques de comprendre la vie compliquée que mène cet oiseau intelligent qui utilise des outils. À l'autre bout de l'échelle, on a aussi équipé des éléphants de radiotransmetteurs pour pouvoir les suivre par radiotracking, aussi bien à des fins scientifiques que pour aider les agriculteurs lorsque leurs champs risqueraient être ravagés par des pachydermes affamés à la recherche d'un repas gratuit.

Les progrès des TI, et les informations disponibles grâce à elles, permettent aux décideurs politiques et aux conservationnistes de mieux gérer les espèces et les écosystèmes menacés. Les TI soustendent aussi des prises de décisions dans d'autres domaines, et spécialement les changements climatiques, en aidant à évaluer leurs impacts réels, par exemple, en comparant la taille des glaciers dans des endroits éloignés, en mesurant la fonte des calottes glaciaires et en prenant à distance la température de la terre. Les TI seront aussi vitales pour comprendre et surveiller la réponse des écosystèmes aux mesures prises. L'utilisation la plus sophistiquée des TI est celle des généticiens qui, sans les technologies 
modernes, auraient peu de chances de comprendre la structure génétique des nombreuses espèces dont le génome a maintenant été décrypté. Des dizaines de bases de données partagées sur les génomes existent maintenant, qui couvrent du riz aux rats, des poissons-zèbres aux hommes, et même l'ornithorynque. Ces bases de données modèles sont un outil de recherche très poussé pour les scientifiques et elles leur permettent de faire des bonds en avant prodigieux dans la sophistication des questions scientifiques auxquelles ils sont capables de répondre.

Malgré ces progrès, le plus grand défi reste de s'assurer qu'une connaissance plus complète de la biodiversité contribue à des politiques et à des prises de décisions plus efficaces. Les TI peuvent et doivent jouer un rôle pivot face à ce défi. Tout indique que ces avancées technologiques vont aller toujours plus vite et donneront accès à des informations importantes toujours plus variées, allant de l'analyse de l'ADN à la richesse des microorganismes des sols ou au calcul de l'empreinte écologique de l'humanité. Tout cela offre la possibilité de construire un avenir technologique qui aidera à améliorer de façon significative la gestion des ressources biologiques, un mariage de la technologie et de la biologie qui pourrait mener à un avenir plus durable.

Il n'y a pas que les aspects matériels des TI qui changent ; il y a aussi les façons dont nous traitons et manipulons les informations. Alors que les ordinateurs sont toujours plus puissants, comme l'avait prédit la loi de Moore (la capacité de stockage des micropuces va doubler tous les 18 mois), notre capacité d'explorer des domaines qui exigent une évaluation très large et très complexe s'est aussi accrue.

Un des facteurs qui limitent la prédiction des impacts sur la nature est l'incertitude associée une donnée qui empoisonne les climatologues depuis des années. De nouvelles méthodologies pour intégrer l'incertitude dans les calculs et les modélisations se font jour, comme les nombres flous et les réseaux bayésiens. Tout ceci est désormais utilisé dans la recherche et la gestion environnementales, y compris dans les évaluations entreprises dans le cadre de la Liste rouge de l'UICN des espèces menacées.

\section{BIOTECHNOLOGIE}

La biotechnologie est étroitement liée à la nouvelle gestion de l'information. On peut ainsi définir toute application de la technologie à des systèmes biologiques. C'est une longue histoire qui remonte à l'utilisation de la levure dans la préparation du pain et la fermentation des boissons alcoolisées. Ces applications anciennes ont été rejointes par d'autres plus modernes, comme les nanotechnologies, le biomimétisme et les modifications génétiques. Certaines de ces nouvelles applications de la biotechnologie ont des effets potentiellement importants et inédits, ce qui exige l'application du principe de précaution.

\section{Nanotechnologies}

Les nanotechnologies signifient que l'on travaille à l'échelle de l'atome, soit environ $10^{-10}$ mètres.

À cette échelle, les matières se comportent de façon très différente que lorsqu'elles sont combinées à d'autres pour former des molécules, des composés, etc. Les nanoparticules sont si petites qu'elles peuvent pénétrer dans des cellules imperméables à des particules plus grosses. Donc, leur usage en cosmétique, par exemple, peut avoir des implications pour la santé. De plus, les nanoparticules ont une surface relativement grande par rapport à leur volume, ce qui augmente leurs propriétés chimiques et électriques et aussi le risque qu'elles entraînent des réactions négatives dans la cellule qu'elles ont envahies.

Si des nanoparticules peuvent être produites naturellement, par exemple par les volcans, les 
nanoparticules fabriquées deviennent un business très sérieux. Les investissements mondiaux dans les nanotechnologies étaient de 10 milliards de USD en 2005 et l'on s'attend à ce qu'ils atteignent mille milliards de dollars en 2011-2015 (Navarro et al., 2008). On en attend des avantages pour les gens dans les domaines de la médecine, de l'électronique et de l'environnement. Par exemple, la capacité des nanoparticules de se lier à des polluants chimiques pourrait réduire la biodisponibilité de ces substances toxiques. Cependant, on a aussi reconnu que les nanoparticules pouvaient avoir des effets toxiques et provoquer, par exemple, une irritation des poumons. Les inconnues entourant l'utilisation des nanoparticules sont nombreuses (Navarro et al., 2008).

Le domaine des nanotechnologies n'est pratiquement pas réglementé aujourd'hui, et peu d'études, s'il y en a, furent faites sur leurs impacts possibles sur la biodiversité. Comme toute nouvelle technologie susceptible d'avoir des conséquences importantes, il faudrait les approcher avec prudence, et l'application du principe de précaution semble indiquée. Sutherland et al. (2008) inscrivaient les nanotechnologies parmi les 25 nouvelles menaces pour la biodiversité. Ils recommandaient, "si l'usage se généralisait ou que ces structures étaient incorporées dans des systèmes " quasi vivants ", de faire appel à de nouvelles approches des risques ». Pour sa part, la Commission européenne (CE) a émis un " code de conduite " pour les nanotechnologies (CE, 2008). Sa section sur la durabilité déclare que :

Les activités de recherches en nanosciences et nanotechnologies sont sûres, respectent les principes éthiques et contribuent au développement durable. Elles concourent aux objectifs de durabilité de la Communauté et contribuent aux Objectifs $d u$ millénaire pour le développement des Nations Unies. Elles ne nuisent ni aux personnes, ni aux animaux, ni aux végétaux, ni à l'environnement et ne constituent pas une menace biologique, physique ou morale à leur égard, à l'heure actuelle comme à l'avenir.

Le Conseil international de la gestion du risque (IRGC) note aussi que, même si les nanotechnologies représentent de grands bénéfices potentiels, elles sont aussi des risques sérieux avec des implications sociales, économiques, politiques et éthiques importantes. L'IRGC suggère que, comme les problèmes soulevés par les nanotechnologies sont plus complexes et profonds que pour de nombreuses autres innovations, les décideurs doivent gérer aussi les incertitudes et les risques associés (IRGC, 2007).

\section{Biomimétisme}

Le mot «biomimétisme » est constitué de la racine grecque " bios", la vie, et de " mimesis », l'imitation. Ce terme s'applique aux applications de modèles et de procédés issus de la nature à des conceptions industrielles ou agricoles destinées à résoudre des problèmes humains. Comme le précise Janine Benyus (1997), c'est une approche qui apprend «de » la nature plutôt que seulement «sur» la nature.

Le biomimétisme est basé sur le principe selon lequel, par l'évolution, la nature a appris ce qui marche, ce qui est approprié et ce qui est durable. La nature comprend des organismes qui volent, qui occupent la terre entière, qui préservent des conditions de vie appropriées et construisent des structures incroyablement complexes. La nature a développé des matières biodégradables comme les colles, produites par des moules, qui fonctionnent sous l'eau, des soies de toiles d'araignées qui sont plus solides que les matières les plus résistantes produites par les hommes, des termitières qui sont capables de maintenir une température constante alors que la température extérieure passe de $40^{\circ} \mathrm{C}$ pendant la journée à 
près de $0^{\circ} \mathrm{C}$ pendant la nuit, et les pattes des geckos qui leur permettent de s'accrocher aux plafonds les plus lisses.

Nous utilisons déjà des applications

biomimétiques dans la vie de tous les jours.

L'invention du Velcro fut inspirée par les bractées de la bardane, et lorsque les frères Wright ont conçu le premier avion à moteur, ils se sont inspirés des ailes des oiseaux. Les panneaux solaires qui alimentent les satellites en orbite sont déployés selon un schéma appris en dépliant des bourgeons minuscules, et les constructions modernes à basse énergie se fondent sur le modèle des termitières. Le travail sur le biomimétisme illustre le rôle d'une nouvelle génération de technologies bien adaptées, basées sur les principes de conception de la nature, pour un avenir durable.
L'importance de la nature pour de meilleurs moyens de subsistance grâce à l'application du biomimétisme est de plus en plus reconnue, et la valeur intrinsèque de la biodiversité comme laboratoire vivant des besoins de l'avenir est toujours plus évidente. La raison d'être de la conservation de toute la nature, stratégie clé de la gestion du risque pour capter la valeur d'option, est largement confortée par les avancées du biomimétisme.

\section{Organismes génétiquement modifiés (OGM)}

Les organismes génétiquement modifiés sont un aspect particulièrement controversé de la modification de la diversité génétique. Ils deviennent de plus en plus présents dans de nombreux pays et sont utilisés dans beaucoup de secteurs, de l'agriculture à la santé et à la fourniture d'énergie. Les Membres de l'UICN

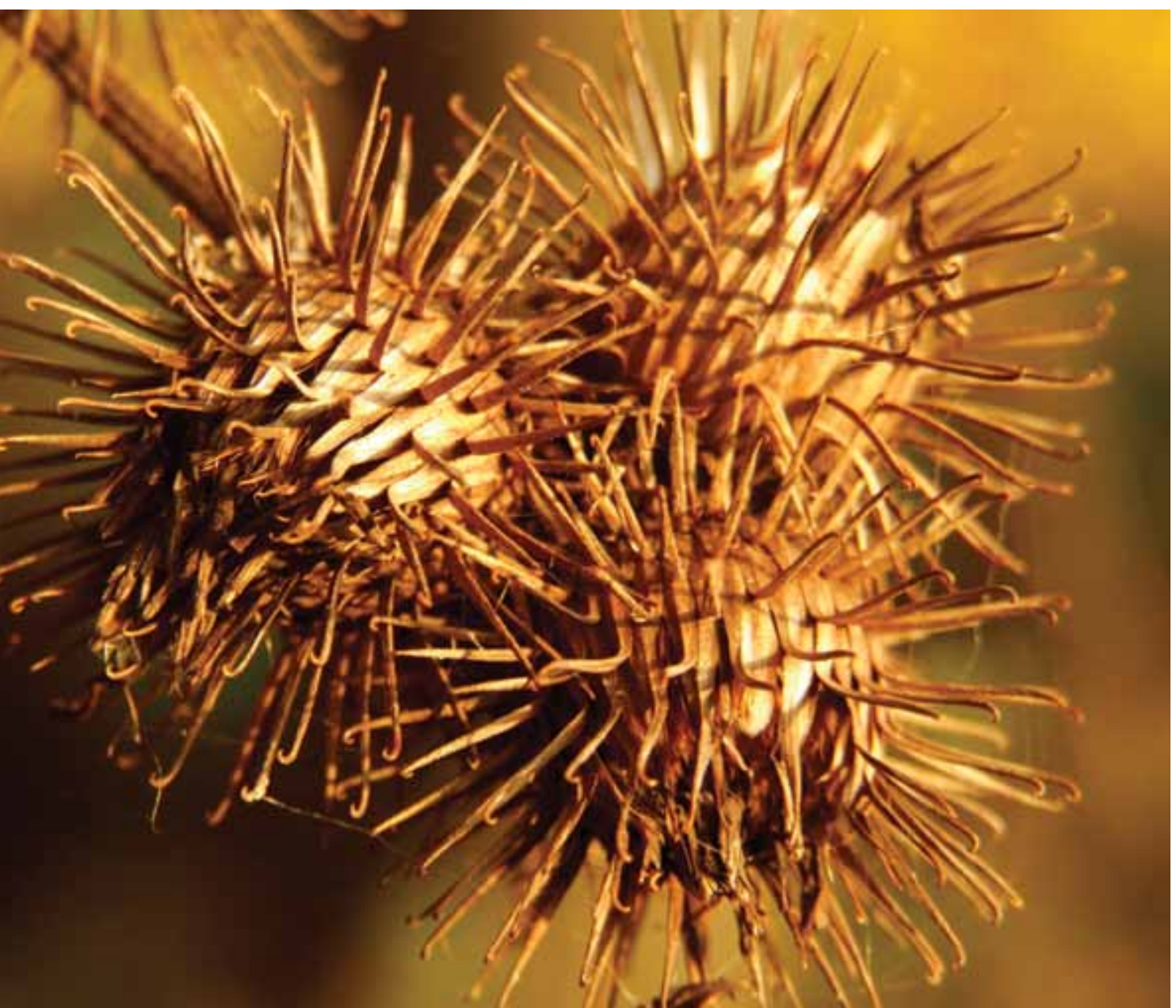


reconnaissent cette tendance croissante et, tout en notant qu'il est possible que les OGM améliorent les moyens de subsistance et favorisent le développement, ils ont exprimé leur crainte concernant les éventuels effets négatifs des OGM sur la sécurité alimentaire et sur l'environnement. Cette crainte se reflète dans la Résolution de l'UICN CMN 3.007 dans laquelle l'Union demande " un moratoire sur les futures libérations d'OGM dans l'environnement jusqu'à ce que l'on ait la preuve quasi-certaine que les OGM sont sans danger pour la diversité biologique et la santé humaine et animale ». Les Membres de l'UICN reconnaissent aussi les développements rapides dans les différents domaines de la technologie génétique et ont demandé des mises à jours continues sur cette question.

Les éventuelles incidences négatives des OGM incluent une réduction de la biodiversité, des risques pour la santé humaine, des conséquences inattendues du transfert de gènes entre plantes et la création de nuisibles ou de mauvaises herbes résistantes à tout contrôle. Les Parties à la Convention sur la diversité biologique (CDB) ont reconnu les bénéfices et les coûts potentiels des OGM dans le Protocole de Carthagène, qui encourage l'utilisation informée et prudente de cette technologie et qui travaille à renforcer les capacités dans tous les pays qui appuient les processus de prises de décisions impliqués. Les Membres de l'UICN ont demandé aux gouvernements de ratifier le Protocole de Carthagène.

Les organisations des Nations Unies responsables de la santé humaine et de la production alimentaire n'ont trouvé aucune preuve, à ce jour, d'impacts négatifs des OGM sur la biodiversité et la santé humaine. En 2003, un examen des recherches entreprises pour évaluer les impacts environnementaux des cultures transgéniques a conclu que le suivi et les expériences étaient insuffisants pour tirer des conclusions à cet égard (Ervin et al., 2003). Même si les scientifiques ont trouvé peu de preuves concluantes d'impacts négatifs directs des OGM sur la biodiversité ou la santé humaine, d'autres questions éthiques doivent être envisagées. Certaines organisations partagent les vues de Via Campesina, un mouvement mondial de paysans agriculteurs, qui croit que la technologie des OGM constitue un risque grave et immédiat pour la sécurité et les moyens de subsistance des paysans agriculteurs (www.viacampesina.org). D'autre part, certains agriculteurs des pays en développement comme la Chine, l'Inde, l'Argentine et le Brésil accueillent les cultures d'OGM les bras ouverts, spécialement le coton, le soja et le maïs.

\section{Géo-engineering}

Le géo-engineering est la modification délibérée de l'environnement pour obtenir des résultats spécifiques liés aux besoins humains. En ce qui concerne les changements climatiques, deux aspects du géo-engineering sont envisagés : la gestion du rayonnement solaire, par exemple par la création d'aérosols sulfatés solaires, et celle des émissions de GES, par exemple par des techniques de capture et de stockage du carbone ou l'emploi de biochar comme puits de carbone (Victor et al., 2009). Les effets collatéraux de ces technologies sont encore à peu près inconnus. $\mathrm{Au}$ moins une technologie de géo-engineering a été testée, la fertilisation de l'océan par du fer pour encourager la croissance d'un phytoplancton qui séquestre le carbone, et elle a fait l'objet de nombreux débats dans les arènes mondiales de la politique environnementale; des gouvernements ont accepté un moratoire sur de nouveaux tests de cette technologie.

Mathews et Caldera (2007), qui étudiaient spécifiquement le problème de la gestion du rayonnement solaire, rapportent que, si les solutions que donne le géo-engineering peuvent apporter quelques atténuations, ces technologies 
masquent aussi des augmentations des émissions de GES. Si les solutions du géo-engineering devaient échouer ou s'arrêter soudainement, il pourrait en résulter des changements climatiques très rapides, avec un réchauffement qui serait 20 fois plus rapide que le rythme actuel. Ils concluent qu'en se fiant uniquement au géoengineering sans effort supplémentaire pour réduire les émissions de carbone, le système climatique mondial serait en grand danger.

\section{Biologie synthétique}

Si certains considèrent encore que la biologie synthétique est simplement une extension du génie génétique, il est en fait bien plus complexe et il implique la fabrication de nouveaux systèmes et fonctions biologiques, qui n'existent pas dans la nature, et la re-conception ou la re-fabrication à des fins utilitaires d'éléments biologiques existants (IRGC, 2008b). Alors que l'ingénierie génétique n'implique classiquement qu'un ou quelques gènes à la fois, la biologie synthétique crée des organismes ou des unités métaboliques entièrement nouveaux. Cette technologie n'en est encore qu'à ses débuts, mais elle a montré qu'il est possible de créer des génomes viraux comme le virus de la polio (Cello et al., 2002) et de reconstruire le virus qui fut responsable de la pandémie de grippe de 1918 (Tumpey et al., 2005).

Comme c'est une branche émergente de la biologie, la plus grande partie des travaux dans ce domaine sont encore loin d'avoir des applications commerciales. Mais ses défenseurs entrevoient des possibilités en bioremédiation (par exemple, pour dégrader des pesticides et enlever des polluants), dans le développement de biosenseurs qui peuvent détecter des toxines chimiques, le développement de bactéries ou de virus qui peuvent identifier les cellules cancéreuses et transporter les agents thérapeutiques à l'endroit requis, le développement de produits pharmaceutiques plus efficaces, la création de microorganismes qui peuvent produire de nouvelles sources d'énergie et d'autres applications qui dépassent l'imagination actuelle.

D'autre part, la biologie synthétique peut présenter de sérieux risques, comme les effets destructeurs inattendus de la dispersion accidentelle dans l'environnement d'organismes synthétisés, comme ceux qui ont été conçus au départ pour la bioremédiation. Recourir à la biologie synthétique pour créer des microorganismes peut entraîner des effets imprévisibles : dans un scénario catastrophe, des organismes nuisibles pourraient être créés délibérément (encore qu'il soit plus facile, de nos jours, d'obtenir des agents pathogènes autrement). D'un point de vue philosophique, il est possible que la biologie synthétique conduise au constat que la plus grande partie de l'évolution se passe désormais dans les laboratoires plutôt que dans la nature, ce qui pourrait présenter des risques importants pour le concept même de nature, et pour la biodiversité.

En 2003, J. Craig Venter et son équipe de chercheurs ont réussi à fabriquer un chromosome entièrement synthétique en deux semaines. Depuis lors, l'Institut Venter est à la pointe de la génomique synthétique pour étudier et répliquer la génétique de la vie (Smith et al., 2003). En 2008, des scientifiques de l'Institut de recherches de J. Craig Venter ont annoncé le premier génome bactérien entièrement synthétique (Mycoplasma genitalium) franchissant ainsi un pas important vers la vie artificielle.

Les outils de la biologie synthétique sont facilement disponibles en ligne dans toute bibliothèque ouverte, le Registry of Standard Biological Parts (http://parts.mit.edu). Des étudiants participent déjà à des compétitions où ils utilisent des « biobriques " pour construire leurs propres systèmes biologiques synthétiques, alors qu'il n'existe encore aucune mesure de réglementation pour garantir que de telles 
expériences ne menacent pas l'environnement

(IRGC, 2008). La biologie synthétique est un domaine qui en appelle à coup sûr au principe de précaution et elle mérite une plus grande attention de la part du domaine de la conservation qu'elle n'en reçoit pour le moment.

\section{TIRER LE MEILLEUR PARTI DE LA TECHNOLOGIE}

\section{D'AUJOURD'HUI EN SOUTENANT L'ENVIRONNEMENT}

Pour relever les défis de la conservation d'aujourd'hui, les nouvelles technologies seront particulièrement importantes car elles nous fourniront les moyens de traiter certaines des principales menaces sur la biodiversité et les services écosystémiques, comme les changements climatiques, la pollution, et les espèces exotiques envahissantes. Dans tous les cas, tirer le meilleur parti de la technologie compatible avec la conservation de l'environnement signifie que dans la prochaine décennie, nous allons devoir soutenir le développement des outils et de la technologie de l'information nécessaires pour gérer efficacement les écosystèmes fragiles et pour assurer des moyens de subsistance durables aux personnes qui vivent dans ces régions. De plus, nous devrons appliquer le principe de précaution pour gérer les nombreuses incertitudes au sujet des impacts à long terme de certaines de ces technologies et adopter certains changements comportementaux fondamentaux pour gérer les incidences de l'utilisation de ces technologies, en faisant attention aux $3 \mathrm{R}$ : réduire, recycler, réutiliser. 


\section{Coopération internationale}

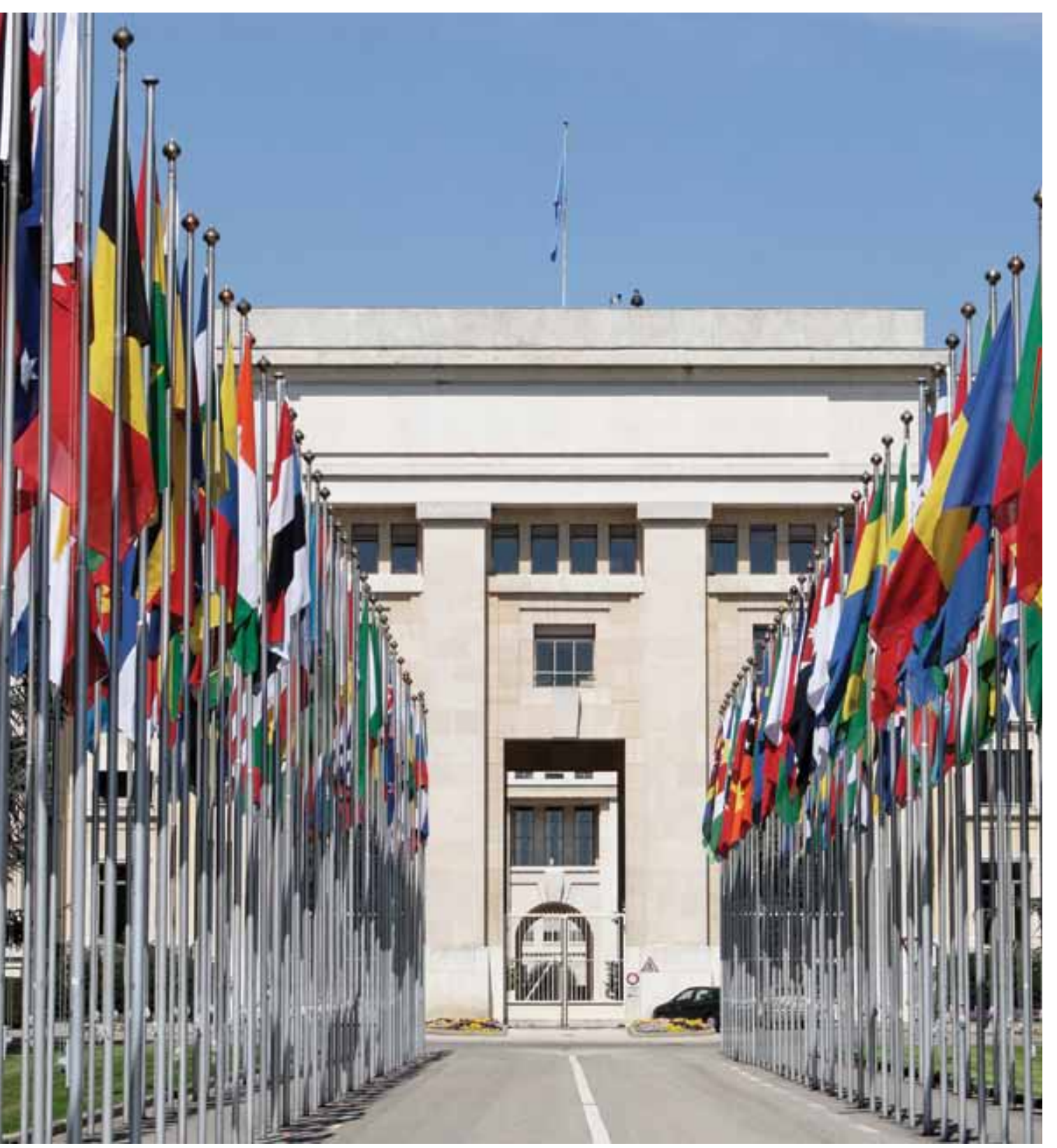




\section{L'agenda politique international d'aujourd'hui est largement centré}

sur les problèmes économiques et sécuritaires, comme les risques d'effondrement financier, le terrorisme, la prolifération des armes et les changements climatiques. La dimension environnementale de ces questions commence à bénéficier d'un peu de temps d'antenne, mais elle se situe encore loin derrière l'attention accordée aux impacts directs sur les hommes du monde entier. Pourtant, on commence maintenant à discuter et à accepter la responsabilité des incidences sur les biens publics mondiaux, y compris sur la biodiversité et les services écosystémiques, dans des secteurs qui vont de la gestion de la pêche aux changements climatiques.

En termes d'agenda international de la biodiversité, la plupart des accords multilatéraux sur l'environnement (AME) et des processus se concentrent sur la mise en œuvre des engagements et les programmes de travail existants. Les nouveaux défis sont, entre autres, les discussions sur le besoin d'un objectif et d'un cadre en matière de biodiversité pour l'après-2010, la négociation en cours pour un régime international d'accès et de partage des avantages (APA) dans le cadre de la Convention sur la diversité biologique (CDB), et des mesures pour s'attaquer au problème de la gouvernance en haute mer, en dehors des juridictions nationales, dans le contexte de l'Assemblée générale des Nations Unies. Celles-ci ont déclaré l'année 2010 Année internationale de la biodiversité, orientant les projecteurs sur les discussions de la 10ème Conférence des Parties à la Convention sur la diversité biologique (CDB COP 10) qui aura lieu au Japon en octobre 2010. La Commission sur le développement durable (CDD) se focalisera aussi sur la biodiversité en 2010 .

Les changements climatiques offrent aux questions environnementales une occasion de devenir un aspect important de ces négociations. Mais de nombreuses contraintes existent avant d'intégrer totalement l'environnement dans ces discussions. Il faut d'abord se mettre d'accord sur le besoin d'harmoniser instruments et discussions, emballer ensemble de façon convaincante biodiversité et changements climatiques (Chapitre 5), renforcer les capacités (techniques et financières) pour mettre en œuvre les décisions prises et mobiliser les volontés politiques pour qu'elles agissent pour le bien du monde. 
HARMONISATION ET SYNERGIE ENTRE

LES ACCORDS INTERNATIONAUX

Au cours de la seconde moitié du 20ème siècle, et surtout après la Conférence de Stockholm et la publication du rapport de la Commission Brundtland en 1987, des centaines d'accords environnementaux furent rédigés et ratifiés.

Les plus remarquables, au point de vue de la biodiversité, comprennent la Convention sur la diversité biologique, la Convention sur les espèces migratrices (CMS), la Convention sur le commerce international des espèces de faune et de flore menacées (CITES), la Convention sur le patrimoine mondial et la Convention de Ramsar sur les zones humides d'importance internationale.

Ce réseau d'instruments a créé, dans certains cas, un cadre très enchevêtré et difficile à appliquer pour la conservation. Par exemple, les tortues imbriquées de la région des Caraïbes font l'objet, à elles seules, de la juridiction de plus de 12 instruments mondiaux (de la CITES à Ramsar en passant par la Convention des Nations Unies sur le droit de la mer - UNCLOS), de plus de sept accords régionaux et de trois accords atlantiques (CITES, 2001). Malheureusement, les obligations et les exigences de tous ces instruments ne sont pas toujours accordées entre elles et cela oblige les Caraïbes à se débrouiller pour identifier le programme de gestion approprié pour les tortues imbriquées.

Dans le même ordre d'idée, alors que la Convention-cadre des Nations Unies sur les changements climatiques (CCNUCC) est l'instrument primordial pour une coopération globale pour les changements climatiques, de nombreux autres accords mondiaux et régionaux reprennent aussi le climat dans leur travail. Au point de vue de la biodiversité, citons la CDB, la Convention des Nations Unies sur la lutte contre la désertification (UNCCD), l'UNCLOS, la CMS, et Ramsar au niveau international (McNeely, 2008). Mais les agendas et les exigences individuels
Encadré 14.1 Définition des terres arides en termes de politique

La CCNUCC définit les zones arides et semi-arides comme des zones, à l'exclusion des zones arctiques et subarctiques, dans lesquelles le rapport entre les précipitations annuelles et l'évapotranspiration possible [l'indice d'aridité] se situe dans une fourchette allant de 0,05 à 0,65 .

La CDB les définit comme les terres arides et subhumides, y compris les régions arides et semi-arides, les prairies, les savanes et les paysages méditerranéens.

varient aussi entre ces instruments, laissant les Parties devant une panoplie un peu étourdissante d'actions à réaliser. Dans certains cas, même la définition du problème ou de sa portée est différente, par exemple la définition de zone aride dans la CCNUCC et dans la CDB (Encadré 14.1).

Plus de 700 accords internationaux concernent l'environnement et l'on n'a établi aucune architecture internationale réelle pour coordonner cette multitude d'accords officiels, ce qui aboutit à des fragmentations et à des duplications, mais aussi à de sérieux problèmes de capacités pour de nombreux pays - le problème que les anglophones qualifient de "treaty congestion". Alors que le nombre de traités augmente, et avec lui le nombre de décisions et de mesures à prendre, les Parties appellent à de meilleures harmonisation et synergie. Parmi les essais pour consolider l'efficacité, l'harmonisation et la synergie, se trouve Tematea (www. tematea.org), un outil en ligne qui donne des informations rapides sur les décisions et les résolutions d'un grand nombre de traités et de conventions en fonction des sujets. Il y a aussi ECOLEX (www.ecolex.org), une plateforme créée par le Centre du droit de l'environnement 


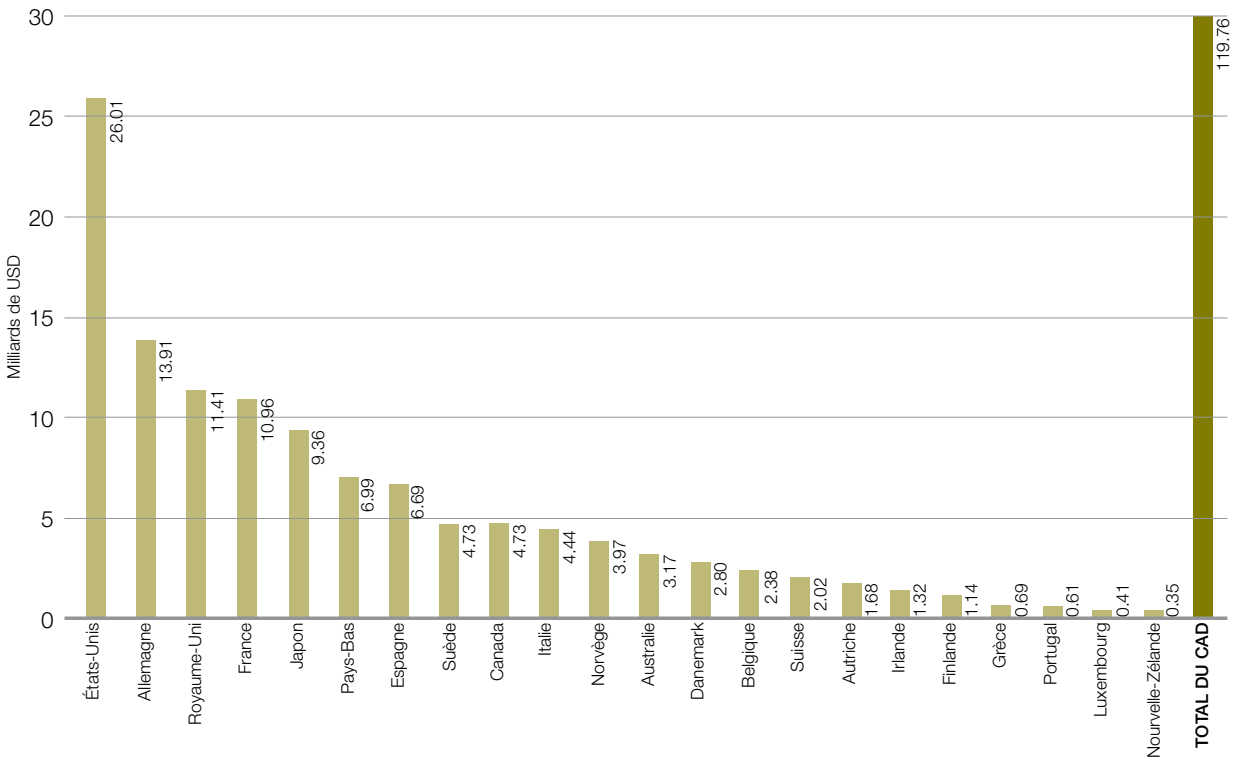

APD nette en 2008 - en pourcentage du RNB

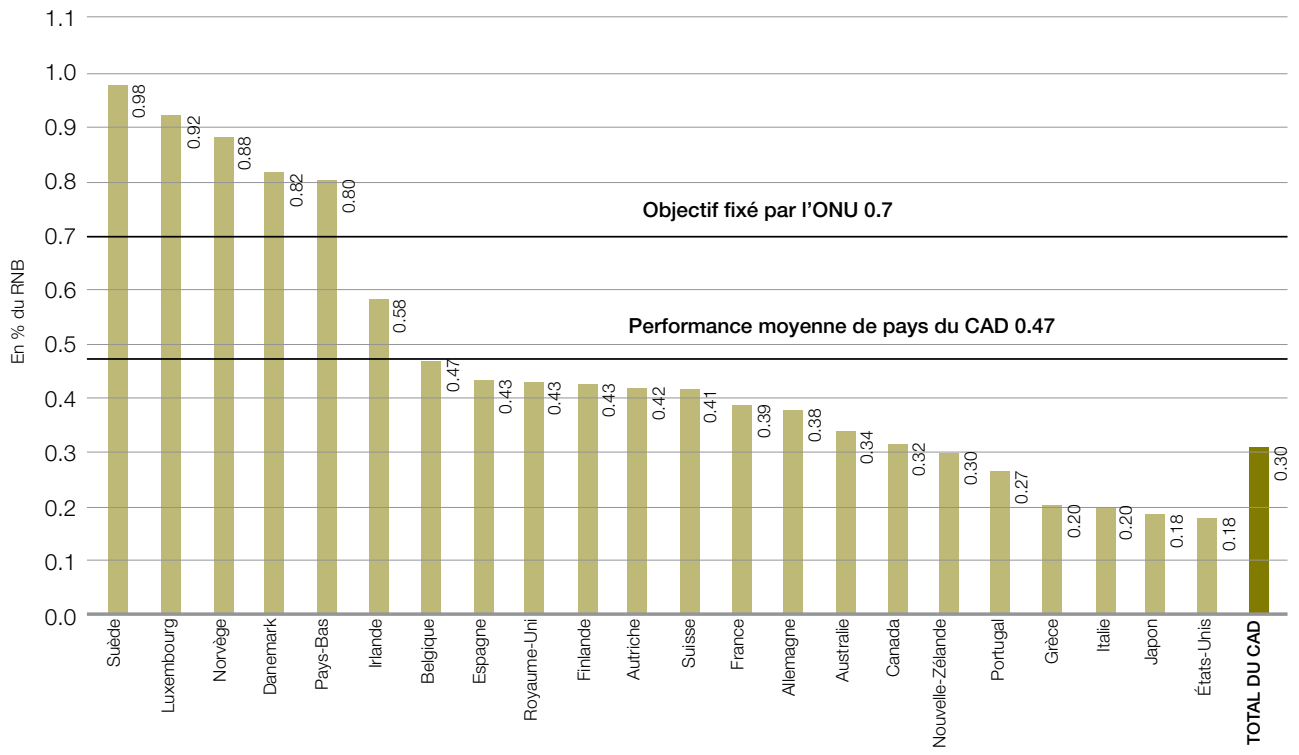

Figure 14.1 Tendances de l'APD (OCDE, 2009)

(CDE) de l'UICN, en collaboration avec le Programme pour l'environnement des Nations Unies (PNUE et l'Organisation des Nations Unies pour l'alimentation et l'agriculture (FAO), qui donne accès à plus de 600 traités multilatéraux et à 45.000 lois et réglementations nationales, qui traitent de la conservation et de l'utilisation des ressources naturelles ainsi que de la contamination de l'environnement par de la pollution ou des déchets. La possibilité d'avis contraires, comme dans le cas de la tortue imbriquée, grandit avec chaque ajout d'instrument et il faut faire attention à assurer une synergie avec ceux qui existent déjà ainsi qu'aux moyens de les mettre réellement en œuvre. 


\section{CAPACITÉS DE MISE EN CEUVRE}

Si l'harmonisation fait certainement partie de la réponse, un autre aspect de la coopération internationale doit être pris en compte : c'est celui des capacités, aussi bien techniques que financières, de respecter les engagements existants. Ceci est particulièrement préoccupant dans les pays en développement dont on attend qu'ils soient des partenaires à part entière dans les accords pris lors de discussions de réunions internationales, mais qui manquent du support et des systèmes sous-jacents qui leur permettraient de participer pleinement aux négociations ou de mettre en pratique les décisions prises.

Le Fonds pour l'environnement mondial (FEM) fut créé pour être le premier mécanisme financier de soutien à la CDB. Entre 1991 et 2006, le FEM a accordé près de 2,2 milliards de USD de subventions et a participé, avec levier financier, à hauteur de 5,17 milliards de dollars au cofinancement de plus de 750 projets pour la biodiversité, dans 155 pays. Ces montants sont insignifiants face aux besoins généraux de la conservation. On estime qu'une conservation mondiale efficace requerrait un investissement de 20-25 milliards de dollars par an (James et al., 2001) - un objectif qui est tout à fait dans les moyens des systèmes financiers actuels, eux qui dépensent des milliards pour renflouer les banques.

En termes de capacité financière, étant donné le rôle de la biodiversité dans le bien-être des hommes, une autre voie de soutien devrait être l'Aide publique au développement (APD) des pays en développement. En 2008, l'APD fut de 119,8 milliards de dollars, c.-à-d. 0,3\% seulement $\mathrm{du}$ Produit national brut (PIB) des pays membres du Comité d'aide au développement de l'OCDE (CAD), beaucoup moins que les $0,7 \%$ sur lesquels ils étaient tombés d'accord (OCDE, 2009 - Figure 14.1). L'APD cible d'habitude les problèmes de développement humain (par exemple, l'éducation et la santé) et pas la conservation. Mais l'OCDE$\mathrm{CAD}$ - étant donné que nous sommes de plus en plus conscients de la dépendance des populations vulnérables vis-à-vis des services écosystémiques de leur environnement - a mis en évidence le rôle de la gestion durable des ressources naturelles dans la croissance "pour les pauvres » et il a recommandé de « fournir une aide à la coopération au développement pour une meilleure gestion des ressources naturelles » (OCDE, 2008).

Cinq pays ont dépassé la cible des Nations Unies des 0,7\% du PIB : le Danemark, le Luxembourg, les Pays-Bas, la Norvège et la Suède. Les augmentations les plus importantes en volume sont venues des États-Unis, du Royaume-Uni, d'Espagne, d'Allemagne, du Japon et du Canada. Pourtant, vu le changement soudain du climat financier mondial en 2008, ce niveau d'investissement pourrait être difficile à tenir. D'autre part, comme les dommages environnementaux causés par les pays riches deviennent plus visibles, les pays en développement qui soufrent de façon disproportionnée de ces dommages ont des arguments plus solides à présenter en faveur d'un appui à la préservation (ou à la restauration) des écosystèmes en bon état (Srinivasan et al., 2008). Les paiements internationaux des services écosystémiques (PES) pourraient être un important moyen de rendre l'économie mondiale plus verte et de faire naitre une collaboration internationale pour la conservation.

Mais tout examen du support de l'APD pour les pays en développement ou du FEM pour la conservation de la biodiversité passe à côté du fait que l'intrant financier le plus important pour ces pays vient de sources d'investissement bilatérales. En 2007, la Conférence des Nations Unies sur le commerce et le développement (CNUCED) a rapporté un montant de près de 2 trillions de dollars d'Investissement direct à l'étranger (IDE), dont 500 milliards furent investis dans 
les économies des pays en développement. Le montant des sources IDE est plusieurs fois celui de l'APD, mais ces chiffres peuvent être très volatils d'année en année et changer rapidement, comme cela s'est passé lors de la chute des économies des "Tigres " asiatiques à la fin des années 1990, et comme cela risque de se poursuivre ces prochaines années lorsque la dégringolade financière due à la crise mondiale des crédits commencera à faire son effet.

Cet investissement étranger direct reflète lui aussi l'existence d'un nombre significatif d'accords bilatéraux. Crawford et Fiorentino (2005) rapportent que les Accords commerciaux régionaux (ACR) sont « une caractéristique majeure et peut-être irréversible du Système commercial multilatéral (SCM) actuel ». Ils suggèrent que les progrès limités des négociations internationales dans le cadre du Cycle de Doha pourraient avoir accéléré le développement d'ACR dans le monde, et particulièrement dans l'hémisphère occidental et la région AsiePacifique (Figure 14.2).

\section{VOLONTÉ POLITIQUE ET OPINION PUBLIQUE}

En fin de compte, le facteur limitatif de toute coopération internationale est la volonté politique, aussi bien de s'accorder sur des décisions, que de soutenir la mise en œuvre complète de ces décisions.

Depuis 1992, la Fondation Asahi Glass mène une enquête sur les problèmes environnementaux et la survie de l'humanité. Les résultats de l'étude pour 2008 (Figure 14.3) portent sur les réponses données par 732 personnes venant de 81 pays et indiquent que $70 \%$ des participants ont cité le réchauffement climatique comme le souci majeur, suivi par les pénuries d'eau (50\%) et la perte de biodiversité (43\%). L'enquête comprend une mesure de la sensibilisation à l'impact des problèmes environnementaux que connaît l'humanité, représentée sur une « Horloge du Jugement dernier » environnemental (Environnemental Doomsday Clock) (qui se dirige vers minuit). En 2008, les participants de toutes les régions, à l'exception du MoyenOrient et de l'Asie, lorsqu'ils ont choisi sur cette horloge l'heure qui correspondait à leur niveau d'inquiétude au sujet de la détérioration de l'environnement, ont en moyenne indiqué 21h33, ce qui était une avance de deux minutes vers minuit, la plus forte accélération annuelle depuis le début de cette étude (Asahi Glass Foundation, 2008).

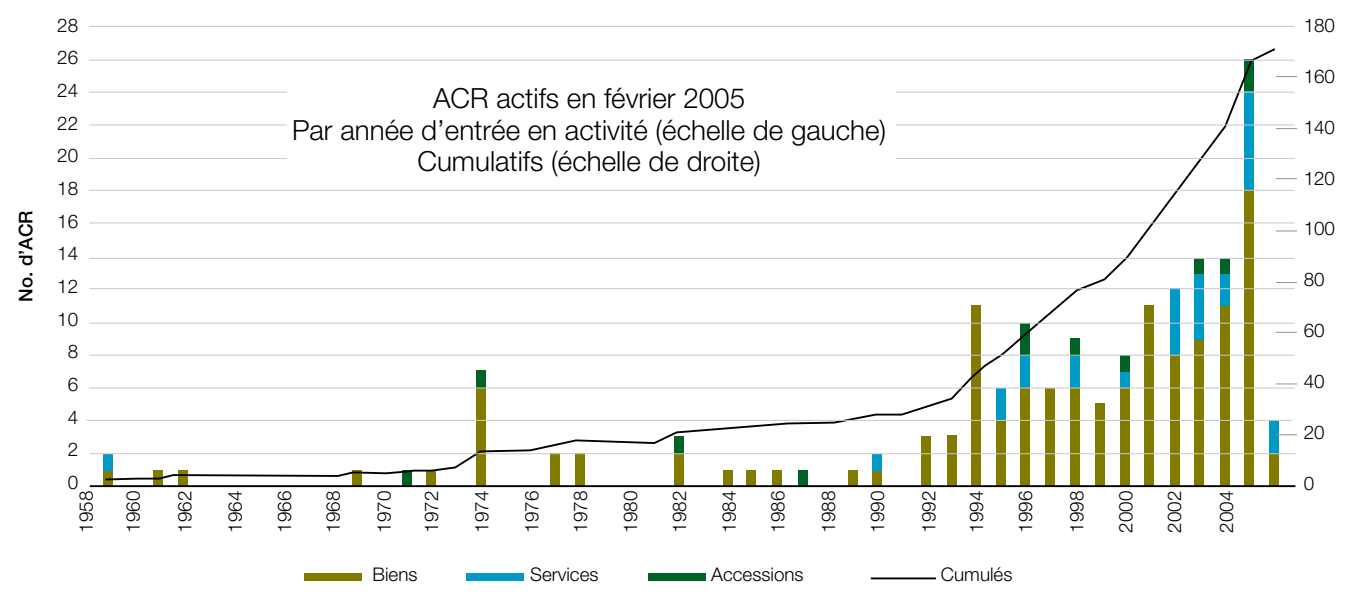


Dans la mesure où la volonté politique reflète l'opinion publique, le facteur limitatif implique aussi en réalité la communication des problèmes environnementaux au public. Le temps, les efforts et l'investissement nécessaires pour mettre le climat à l'agenda politique doivent être répétés pour la biodiversité.

Pour la communauté environnementale, faire reconnaître le rôle de la conservation dans des discussions qui ne concernent pas l'environnement est un moyen important pour y engager les décideurs et susciter cette volonté politique et le ferme soutien du public. Les nouvelles façons de penser le développement et l'aide au développement signifient qu'il faut accorder de l'attention aux programmes de réduction de la pauvreté pour s'assurer que l'environnement est intégré systématiquement dans le courant dominant du développement. Il faudra clarifier les besoins de bonne gouvernance pour réaliser une conservation et une gestion des ressources naturelles effectives et équitables, spécialement au niveau des communautés.
L'intérêt des donateurs pour ces aspects de la gouvernance fut révélé par exemple par le lancement de la Commission de haut niveau sur le renforcement du pouvoir légal des pauvres, en marge du Sommet mondial de 2005

(PNUD, 2005).

Les engagements pris en dehors des accords environnementaux multilatéraux liés à la biodiversité, comme l'engagement du Sommet mondial du développement durable (SMDD) envers la Cible de la Biodiversité 2010 et audelà, pourraient être critiques pour assurer la conservation et le développement à l'avenir. Néanmoins, les progrès accomplis vers l'atteinte de la Cible 2010 et des Objectifs du millénaire pour le développement (OMD) en général, ont été lents (Chapitre 1). Pour atteindre ces cibles, la communauté internationale doit faire des efforts sans précédent.

Dans certains cas, la volonté politique peut être plus facilement acquise au niveau régional ou transfrontalier. Un grand nombre de processus

$\begin{array}{lllllllll}0 & 10 & 20 & 30 & 40 & 50 & 60 & 70 & 80(\%)\end{array}$

Problèmes environnementaux généraux

Réchauffement climatique

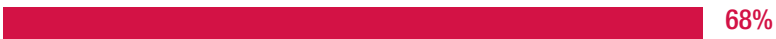

Pollution de l'air, contamination de l'eau, pollution des cours d'eau/océans

Pénuries d'eau, problèmes alimentaires

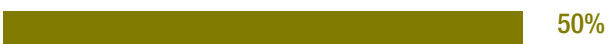

Déforestation, désertification, perte de biodiversité

Modes de vie des gens, problèmes liés à l'eau

Problèmes environnementaux et activités liées à l'économie/ au commerce

Population, pauvreté, statut des femmes

Autres

Inconnus 
et d'institutions régionales forment déjà une part importante de l'agenda de l'environnement et du développement durable. Citons par exemple la Convention africaine, la Convention de Barcelone, la Comisión Centroamericana de Ambiente y Desarrollo (CCAD), la Conférence sur les Ecosystèmes de Forêts Denses et Humides d'Afrique Centrale (CEFDHAC), la Stratégie paneuropéenne pour la protection de la diversité biologique et paysagère (PEBLDS), le Nouveau partenariat pour le développement de l'Afrique (NEPAD), le Mémorandum d'accord sur la conservation et la gestion des tortues marines et de leurs habitats de l'océan Indien et de l'Asie du sud-est (IOSEA), le Programme pour l'environnement des Caraïbes, l'Amazon Treaty Cooperation Organization, et la Free Trade Area for the Americas (FTAA). L'importance de ces processus a été largement reconnue au niveau mondial par des initiatives diplomatiques (ex. SMDD, CDD et le forum des Nations Unies sur les forêts), même si les gouvernements débattent encore pour trouver des modèles de coordination et de collaboration efficaces entre le niveau régional et le niveau mondial. Le grand public ignore souvent ces processus, ce qui suggère que le support du public est plus souvent supposé que systématiquement élaboré.

Les mécanismes pour susciter la volonté politique et publique à un niveau plus local incluent les aires de conservation transfrontalières, comme l'aire de Conservation Transfrontalière du Grand Limpopo, et la gestion d'écosystèmes à l'échelle des paysages (ex. bassins de rivières), ce qui signifie souvent une coopération et une collaboration internationales.

\section{L'AVENIR DE LA COOPÉRATION INTERNATIONALE}

Les défis actuels à relever pour arriver à une synergie des instruments légaux et des capacités de les mettre en ouvre laissent penser que pour être plus efficace à l'avenir, la coopération internationale devra poser un regard nouveau sur les mécanismes actuels et examiner de nouvelles approches pour atteindre les objectifs fixés.

Un «quick win", un « gain rapide " possible consisterait à coordonner durant la prochaine décennie les objectifs et les extrants des nombreuses réunions importantes en matière d'environnement et de développement. De 2009 à 2015, de nombreuses réunions politiques internationales sur l'environnement (Conférence des Parties (COP) à la CCNUCC 15 et au-delà, COP CDB 10, 11 et 12, Rio + 20, OMD 2015) se tiendront en plus du programme régulier du G8 et de l'Organisation mondiale du commerce (OMC) (qui devraient aussi parler des problèmes environnementaux). Si les capacités investies séparément dans tous ces événements pouvaient être focalisées sur des objectifs communs, il y aurait plus de chances que des solutions intégrées, traitant des défis environnementaux, sociaux et économiques, en résultent.

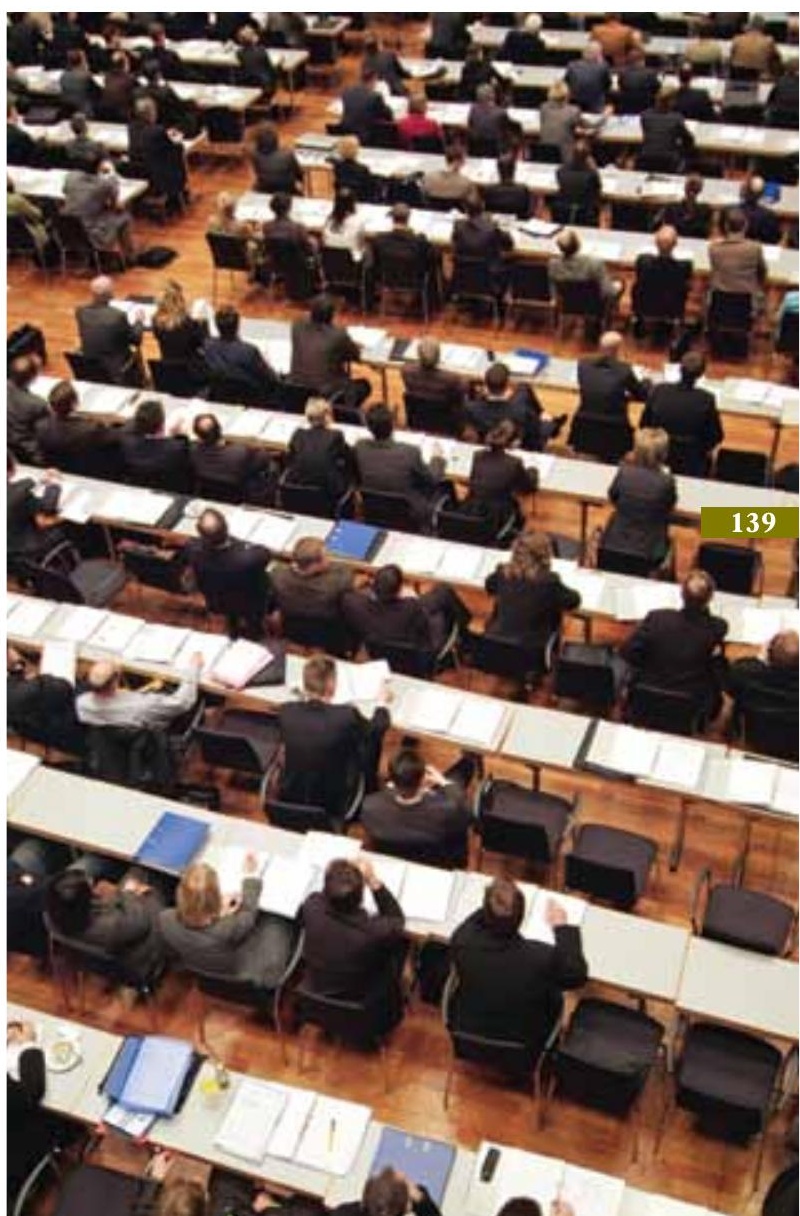


Les défis de la mise en œuvre et de

l'application d'une loi environnementale (et des engagements internationaux en général) mènent à penser qu'il faut reconsidérer la fiabilité des mesures juridiques. Il ne s'agit pas seulement des contraintes de la mise en œuvre et de l'application, mais aussi des techniques législatives qui sont traditionnellement à l'origine des réglementations en matière d'environnement et qu'il faudrait remettre en question. Tout en restant un outil central, la législation est de plus en plus complétée par des mesures plus souples, et d'abord des instruments économiques qui donnent des incitants pour atteindre les objectifs souhaités. Cela comprend des concepts comme le paiement pour les services écosystémiques (PES), qui permet des compensations par le biais d'arrangements statutaires ou contractuels entre acheteurs et vendeurs de services écosystémiques (Chapitre 4). Cette tendance inclut aussi le recours à des approches basées sur les droits, qui devraient accorder plus de poids à la mise en œuvre d'approches traditionnelles fondées sur la responsabilité des états, dont on attend qu'ils remplissent leurs engagements vis-à-vis de leurs citoyens et de la santé des écosystèmes dont ils dépendent.

De nouveaux droits et responsabilités en matière de bonne gouvernance résultent de la reconnaissance du fait que, de plus en plus, les gouvernements ne sont pas les principaux vecteurs de changement. Au niveau mondial, le rôle des industries (évident dans l'augmentation des IDE) a des répercussions importantes sur l'environnement et sur le bien-être humain. En intégrant les instruments économiques "plus souples » qui favorisent la conservation internationale, comme le paiement des services écosystémiques (Chapitre 12), ainsi que des approches basées sur les droits et la responsabilité sociale des entreprises (RSE), on favorisera la présence du secteur privé sur la scène internationale de la conservation.
De nombreux problèmes environnementaux rencontrés par l'humanité sont des problèmes mondiaux qui exigent des efforts internationaux concertés pour obtenir des solutions positives. Durant la prochaine décennie, la communauté de la conservation devra promouvoir des synergies entre les accords multilatéraux et avec tous les nouveaux instruments qui pourraient être mis au point. Il faudrait placer en haut des agendas de chacun l'appui à une mise en œuvre complète des accords existants, via le renforcement des capacités et l'engagement des toutes les parties prenantes, spécialement celles du monde des affaires. Enfin, s'il était possible d'ajouter aux outils disponibles que sont les accords contraignants et la législation restrictive des options volontaires et des incitants positifs, l'engagement actif dans la conservation aurait tout à y gagner. 


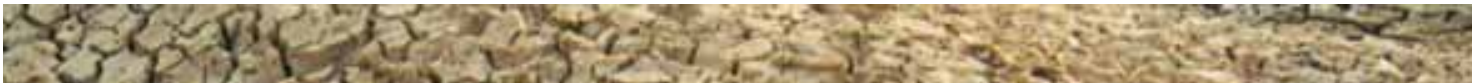

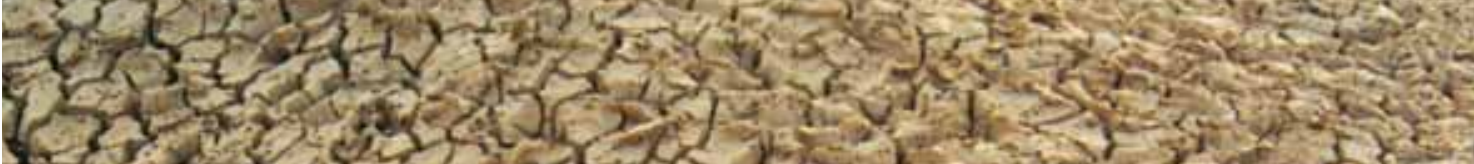

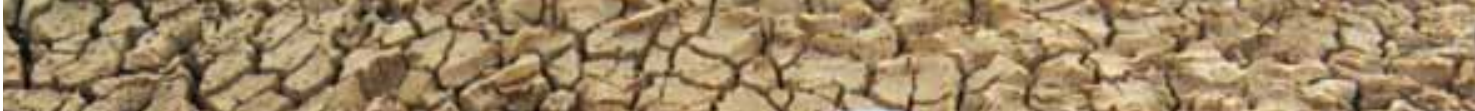

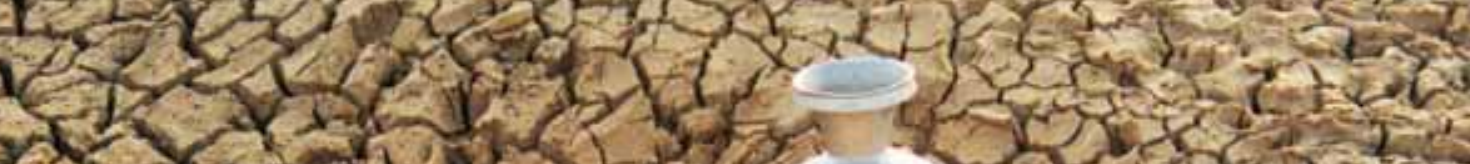

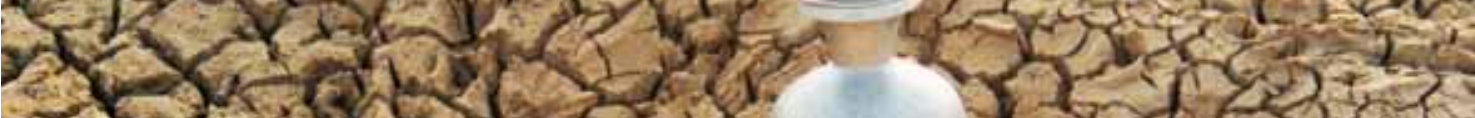

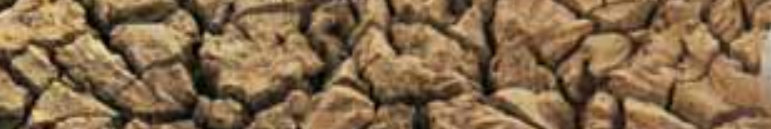

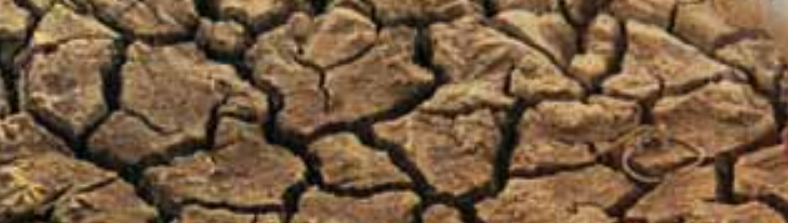
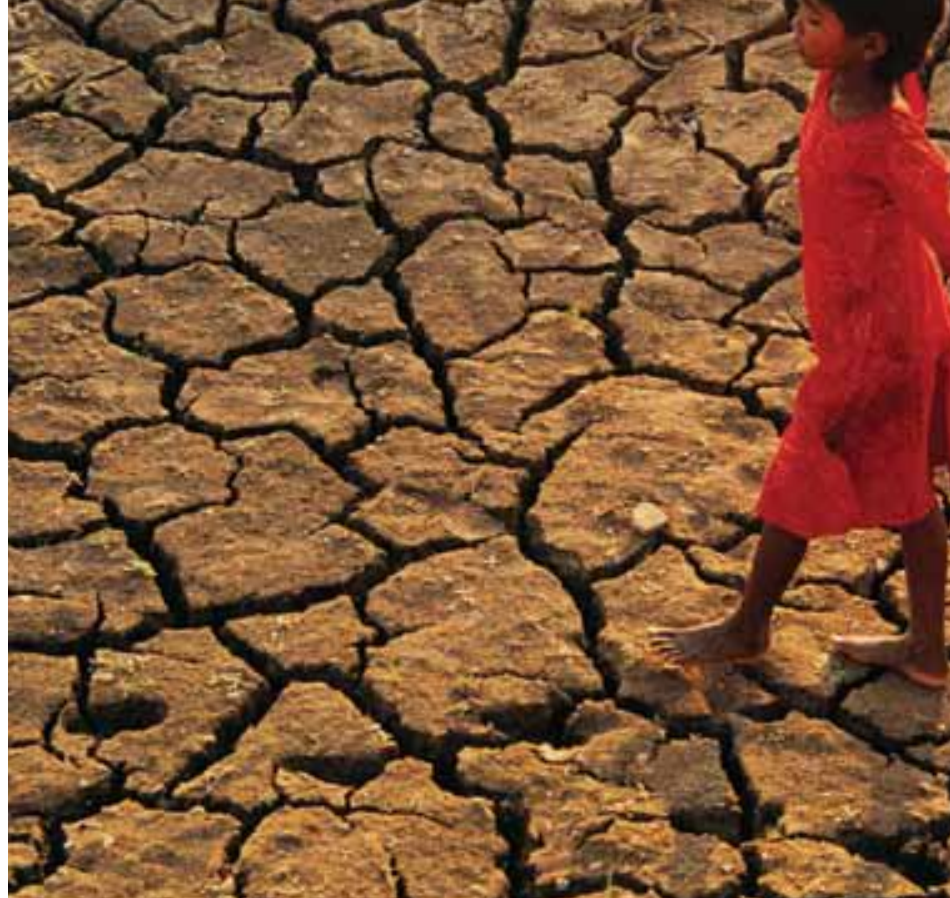

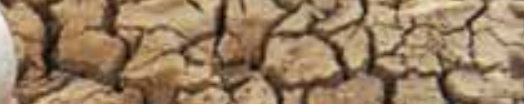

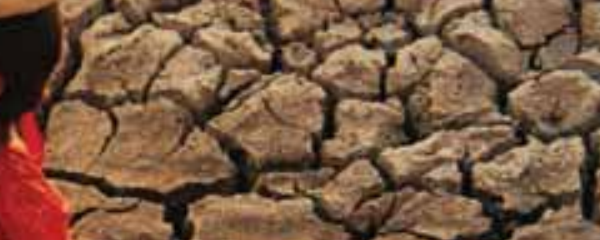

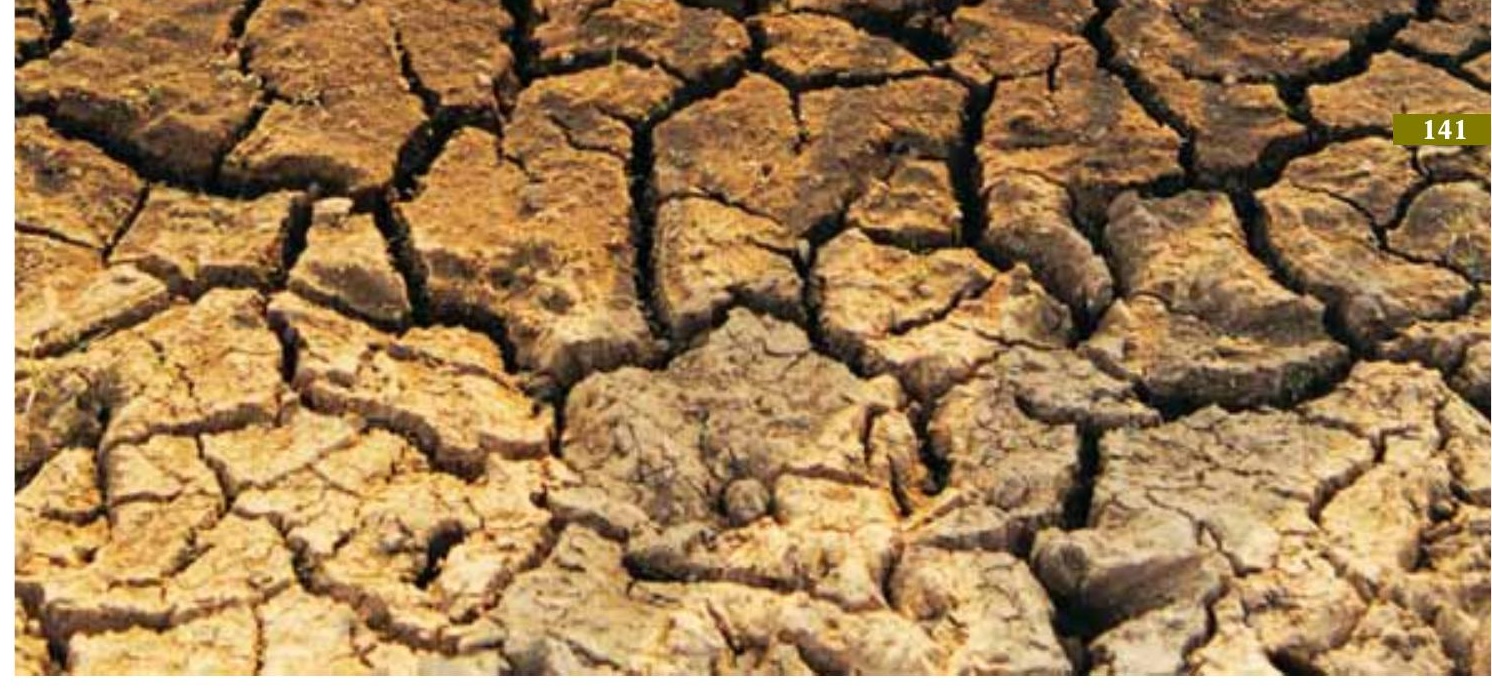


15. Travailler avec le secteur privé

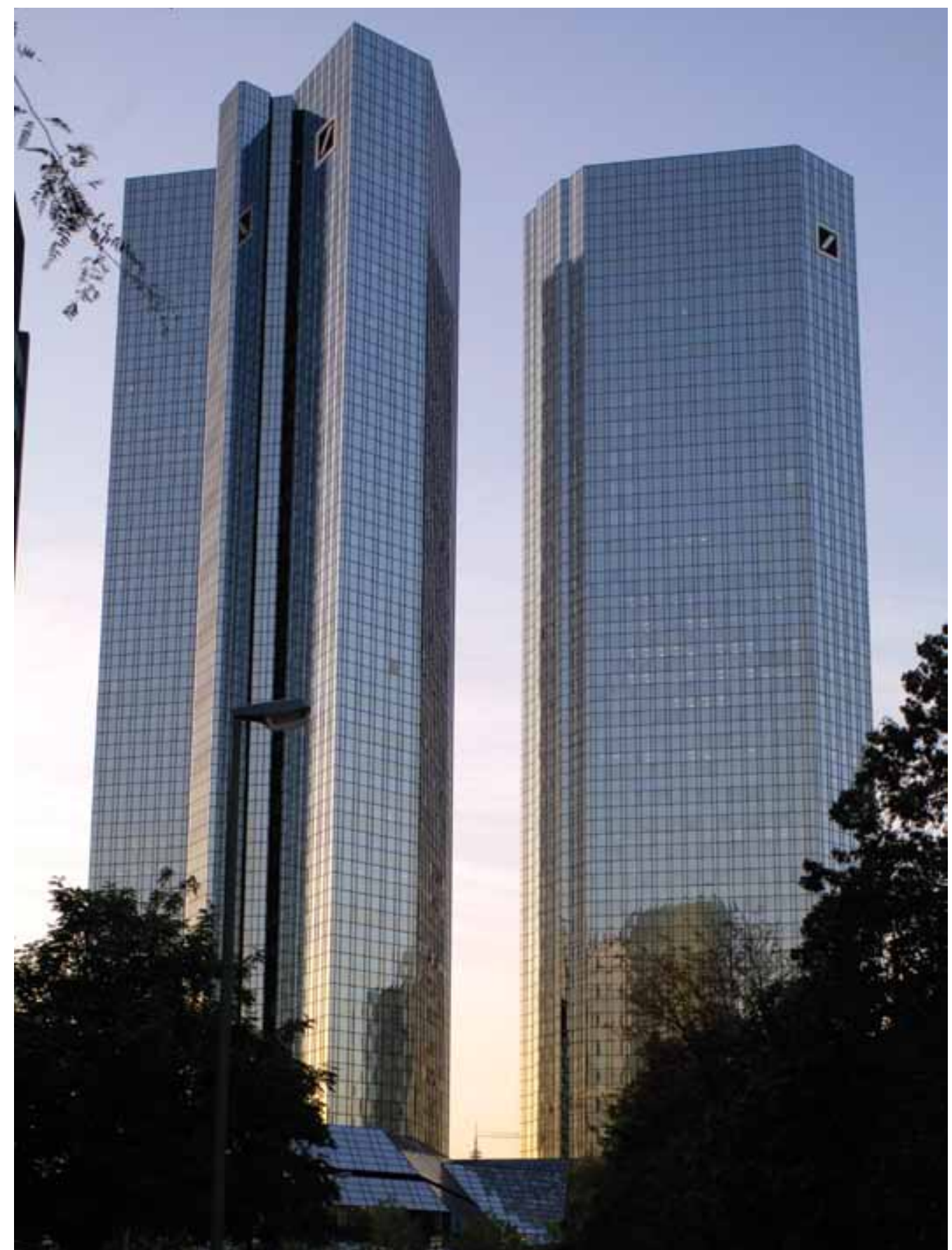


Alors que des banques s'écroulent, que les marchés monétaires se figent et que les politiciens se chamaillent sur les termes d'un sauvetage (ou d'un rachat), de plus en plus de personnes se penchent sur les résultats financiers et la stabilité économique de leur propre avenir, et moins sur des perspectives comme le rôle des marchés et des entreprises dans la conservation de la biodiversité. II est pourtant toujours plus évident que les marchés et les entreprises, vus jadis comme un " problème » de conservation par les environnementalistes, devront être une bonne partie de la solution.

Les entreprises qui pourraient tout particulièrement bien avoir des impacts sur la biodiversité se classent dans les quatre catégories suivantes :

- Les industries à forte « empreinte " (exploitation minière, pétrole et gaz, automobile et fournisseurs d'énergie)

- Les industries qui dépendent de la biodiversité (agriculture, foresterie, pêche, chasse et commerce de vie sauvage)

- Les services financiers (banques, assurances et autres intermédiaires financiers)

- Les entreprises « vertes » (agriculture biologique, coupes de bois à faible impact, énergies renouvelables, tourisme-nature, commerce équitable).

Aujourd'hui, divers secteurs, appartenant ou non au monde des affaires, adoptent des approches variées pour essayer de transformer les pratiques des entreprises, le marché des matières premières et les relations entre entreprises et associations de producteurs, dans le but d'arriver à une meilleure durabilité environnementale.
Pendant le Forum de Barcelone, plusieurs secteurs des affaires ont fourni des thèmes de discussions tels que la pêche (Chapitre 17), l'énergie (Chapitre 7), l'agriculture (Chapitre 20), les industries extractives et le tourisme.

Le lien entre les industries extractives et la conservation est très émotionnel et il est un sujet d'inquiétude intense pour les Membres de l'UICN depuis des années, particulièrement en ce qui concerne les impacts sur les communautés locales et autochtones. Le Conseil international des mines et métaux (CIMM) (2006) a mis au point des Orientations de bonnes pratiques pour l'exploitation minière et la biodiversité qui donnent des informations pour toutes les étapes des opérations et incluent des orientations spécifiques pour la consultation des parties prenantes. Plusieurs résolutions (CMN 4.084, 4.087, 4.088, 4.089 et 4.090) ont été adoptées à Barcelone, qui visent les impacts de l'exploitation minière sur la biodiversité aux niveaux régional et mondial, et plus spécifiquement les impacts sur les aires protégées et sur les communautés locales et autochtones. 
L'Organisation mondiale du tourisme des Nations Unies (OMT) a rapporté que 924 millions de touristes avaient fait un voyage international en 2008 et elle prévoit que ce nombre sera porté à 1,6 milliard en 2020 (OMT, 2009). Le Partenariat pour les Critères mondiaux d'un tourisme durable (GSTC), qui est une coalition de 32 organisations dont l'UICN, a touché près de 100.000 parties prenantes du tourisme, analysé plus de 4500 critères venant de plus de 60 certifications existantes et d'autres ensembles de critères volontaires, et il a reçu les commentaires de plus de 1500 particuliers, pour développer les Critères mondiaux de tourisme durable (Partenariat GSTC, 2008). Ils représentent les normes minimales que toute entreprise touristique doit aspirer à atteindre pour protéger et préserver les ressources naturelles et culturelles du monde tout en s'assurant que le tourisme fait tout ce qu'il peut pour réduire la pauvreté (http://www.sustainabletourismcriteria. org). L'UICN, en collaboration avec les hôtels Accor et l'Association internationale des hôtels et des restaurants, a aussi lancé Biodiversité : mon hôtel agit, des lignes directrices pour l'utilisation durable des ressources biologiques dans ce secteur (UICN, 2008a).

De plus, un secteur émergent de l'« entreprise de la biodiversité » - des entreprises commerciales qui génèrent des profits grâce à des activités qui préservent la biodiversité, qui utilisent des ressources biologique de manière durable et qui partagent équitablement les bénéfices réalisés de cette façon - suscite une attention croissante. Bishop et al. (2008) affirment que le défi actuel de la biodiversité est de réorienter les incitants économiques qui stimulent l'investissement, la production et la consommation privés, et de faire de la conservation de la biodiversité une possibilité de business viable en soi.

Le défi que la dégradation environnementale pose à tous ceux-ci comprend la raréfaction de l'eau, les changements climatiques, le changement des habitats, les espèces envahissantes, la surexploitation des océans et l'eutrophisation. Les tourments actuels des marchés, joints aux défis environnementaux, rendent encore plus pertinente la promotion d'une collaboration entre le secteur public, le secteur privé et les gouvernements dans la protection de la biodiversité et des services écosystémiques qui constituent une partie intégrante de nombreuses opérations commerciales (Encadré 15.1). Les organisations de conservation peuvent aider à mettre en place des mesures/plans de gestion de la biodiversité pour les entreprises dont l'empreinte est importante, comme la réhabilitation de sites miniers. Cette approche ne minimise pas seulement les impacts de l'exploitation minière sur la biodiversité, elle

Encadré 15.1 Le WWF et Coca-Cola - un partenariat pour la conservation de l'eau

En 2006, la Société Coca-Cola et ses sociétés franchisées utilisaient approximativement 290 milliards de litres d'eau pour la production de leurs boissons, dont 114 milliards étaient contenus dans les boissons vendues autour du monde tandis que 176 milliards de litres servaient lors des différentes étapes de conditionnement: lavage, rinçage, réchauffement et refroidissement.

En 2007, Coca-Cola et le WWF ont créé un partenariat qui agit aussi bien sur une utilisation plus efficace de l'eau que sur la réduction des émissions de carbone lors de toutes les opérations de la Société et qui aide celle-ci à encourager des pratiques agricoles durables. De plus, Coca-Cola et le WWF vont collaborer dans des projets pour préserver certains bassins d'eau douce.

Source: WWF, 2007 
aide aussi l'entreprise à obtenir une licence pour travailler dans un nouveau projet.

Le premier objectif d'une société est de faire du profit, mais le secteur privé reconnaît de plus en plus les effets délétères de certaines de ses activités sur l'environnement et le fait que ces impacts sont un risque pour sa propre réussite à long terme. Les entreprises trouvent qu'en accordant plus attention au fait d'être "vertes", elles peuvent aussi accroître leur efficacité dans la mesure où des pratiques judicieuses peuvent rendre leurs activités à la fois plus vertes et susceptibles de mieux marcher. En effet, on a découvert que les sociétés soucieuses de durabilité avaient mieux réussi pendant la crise financière (AT Kearney, 2009). Les bénéfices potentiels d'une entreprise ayant une philosophie de développement durable sont une des questions explorées par le Conseil mondial des affaires sur le développement durable (WBCSD - www.wbcsd.org), une association mondiale de quelque 200 sociétés, dirigée par un de leurs PDG, qui examine le rôle de l'industrie dans le développement durable.

Tant du côté de l'offre que de celui de la demande, les entreprises ont des raisons de développer et de mettre en œuvre des programmes de Responsabilité sociale des entreprises (RSE). Comme le notent Lyon et Maxwell (2008), du côté de la demande, les produits respectueux de l'environnement sont un business en croissance ; les investisseurs poussent les sociétés à adopter des pratiques "vertes ", et les employés préferent travailler pour des entreprises qui « font du monde un meilleur endroit». Du côté de l'offre, les efforts environnementaux sont souvent plus rentables que d'autres options et ils améliorent à long terme l'accès aux ressources nécessaires à leur production. Le fait, par exemple, de convaincre les clients d'un hôtel de consommer moins d'eau pour favoriser la conservation allège aussi la facture d'eau de l'hôtel. Néanmoins, Margolis et al. (2007) ont examiné le lien entre les performances sociales des entreprises et leurs performances financières et n'ont trouvé qu'une très faible relation, mais elle était positive.

Les sociétés peuvent aussi prendre une autre mesure, à savoir s'engager dans des programmes environnementaux volontaires. Selon que ces programmes font l'objet d'une auto-évaluation ou d'une évaluation externe, leur impact sur la société est très variable (Darnall et Sides, 2008).

De grandes entreprises multinationales peuvent avoir de plus solides raisons de « passer au vert » en raison de l'influence de leur notoriété sur leurs activités et des risques sur leur réputation, mais aussi parce qu'ils ont le pouvoir de faire changer les choses. Une grande compagnie qui a des pratiques environnementales contestables risque d'être critiquée publiquement. Les compagnies multinationales ont des normes mondiales qu'elles se doivent de respecter. C'est à ce titre de leaders du secteur que les grandes sociétés ont un rôle positif à jouer. Elles peuvent offrir des procédures et des lignes directrices, des formations et de la sensibilisation.

Pour les organisations de conservation, le défi consiste à voir comment impliquer aussi les petites et les moyennes entreprises (PME). Les PME n'ont pas souvent les ressources ou les capacités nécessaires pour faire des efforts de conservation, ou alors elles n'ont pas la réputation internationale, ou même nationale, qui ferait que la démarche en vaudrait la peine ou qui la rendrait politiquement nécessaire.

Les nouveaux outils ou mécanismes pour aider les entreprises à s'engager de façon constructive dans la conservation comprennent des mesures d'implication dans la mitigation ou encore un support proactif de la conservation. Le Chapitre 12 présente d'autres discussions sur les outils d'une économie verte, comme les compensations, 
les paiements pour les services écosystémiques (PES), les incitants et les subsides.

\section{ÉTABLIR DES RELATIONS AVEC DES ENTREPRISES}

L'UICN a développé des lignes directrices opérationnelles pour travailler avec le secteur privé (UICN, 2009a) et son engagement en la matière ne date pas d'hier. Les organisations de conservation ne peuvent ignorer ou oublier le secteur privé et son énorme impact sur la biodiversité et les services écosystémiques. S’il est important de prendre la parole pour sensibiliser et exercer des pressions, l'engagement constructif est une autre stratégie, complémentaire. L'UICN et les autres organisations de conservation peuvent influencer, encourager et aider à améliorer les pratiques commerciales, avec comme objectif d'améliorer les politiques et les pratiques des industries toutes entières. L'UICN et beaucoup de ses Membres font cela en travaillant avec des acteurs industriels pour améliorer les normes environnementales, et en fin de compte, les réglementations gouvernementales. En s'engageant auprès d'une compagnie, l'UICN peut créer un point d'entrée vers tout un secteur.

Lorsqu'ils envisagent de s'engager avec des sociétés, les conservationnistes doivent d'abord comprendre que les entreprises ne fonctionnent pas comme des ONG ou comme des gouvernements. Pour établir une relation, il faut d'abord comprendre comment elle pourra être bénéfique pour les deux parties. Les questions que les sociétés se posent lorsqu'elles envisagent de travailler avec une organisation de conservation, c'est ce que sont des partenariats verts, pourquoi être plus vert et comment être plus vert.
Comme toute autre organisation qui inaugure une relation avec une société, l'UICN doit être un ami critique et non la promesse d'une approbation sans condition. L'UICN en tant qu'organisation de membres est régie par ses membres, qui sont des gouvernements et des ONG. De nombreux Membres se sont dits inquiets de ces partenariats parce qu'ils y décelaient un manque de transparence. Une telle relation risque aussi de détourner une ONG de sa mission et de sa raison d'être. La principale réticence des conservationnistes vient du fait qu'ils craignent de faire partie d'un "blanchiment écologique ", si une société utilise l'organisation de conservation pour impliquer qu'elle mène des actions de conservation positives alors que cela ne reflète pas la réalité. Pour empêcher cela, il est essentiel que le partenaire " vert » soit professionnel, objectif et transparent dans ses négociations avec ses partenaires du secteur privé. Du point de vue du secteur privé, cela est aussi vital parce que cela garantit la crédibilité du partenaire; si le partenaire « vert » n'est pas crédible, la relation perd toute valeur.

Il peut être contestable de travailler avec le secteur privé et il faut prendre certaines précautions, mais de nombreuses ONG membres de l'UICN sont déjà engagées dans des relations productives avec le secteur privé, dans toutes les régions du monde. Les gouvernements membres de l'UICN appuient en général la collaboration entre ONG et sociétés, parce qu'ils croient que les deux parties peuvent en tirer profit et que cela entraîne une meilleure conservation et de meilleures pratiques commerciales. Le premier avantage que les ONG perçoivent 


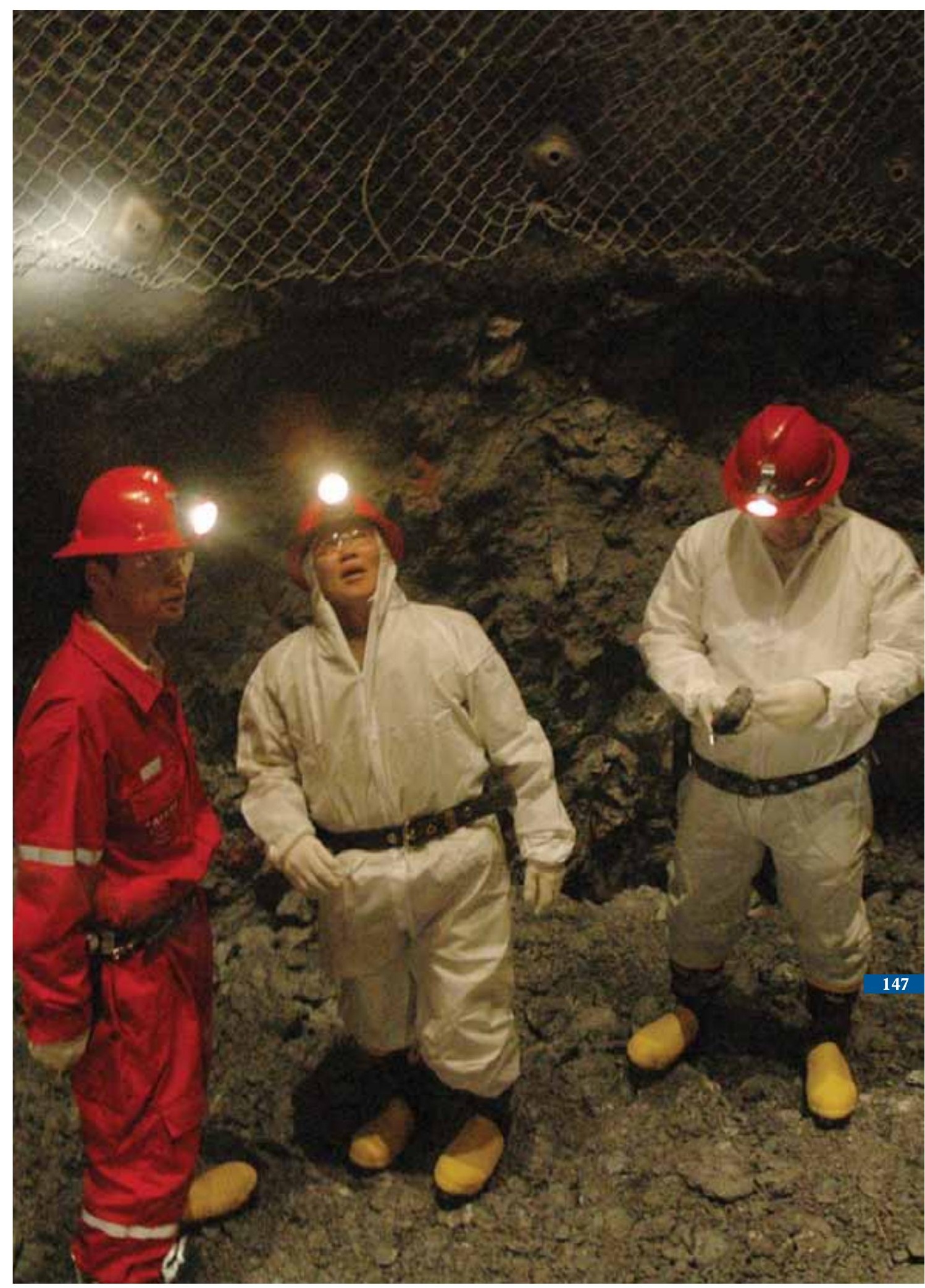


lorsqu'elles s'engagent dans ce type d'accords, c'est le pouvoir qu'ont les sociétés d'influencer les parties prenantes et donc de contribuer à la conservation sur une grande échelle. Ce pouvoir et cette influence peuvent être séduisants, mais la relation peut facilement dégénérer si l'ONG et la société n'observent pas de rigoureuses lignes directrices. Si elles ne le font pas, les bénéfices à long terme de l'engagement risquent d'être perdus, dévalorisant ainsi la réputation des deux parties.

Le type de relation le plus facile entre une organisation de conservation et une entreprise est souvent lié à des accords de sponsoring, comme les logos, mais en s'engageant dans ce genre de relation, les associations risquent de se rendre suspectes de "blanchiment écologique ". De même, le soutien d'organisations de conservation à des programmes de RSE (c'està-dire des mesures autonomes qui vont audelà des exigences légales pour la protection environnementale et sociale) a été critiqué, sous prétexte que ces organisations aidaient au «blanchiment écologique » de la société. En effet, la RSE est un programme volontaire centré sur des pratiques durables; elle ne signifie pas obligatoirement que l'on intègre la conservation dans les affaires et elle n'est d'ailleurs pas forcément intégrée dans les pratiques générales ou les décisions d'affaires de l'entreprise.

Des relations plus engagées pourraient aller des projets de conservation réalisés en partenariat commercial au soutien et à l'analyse des activités des sociétés. Depuis 2004, par exemple, l'UICN travaille avec Sakhalin Energy de Russie, un consortium de Gazprom, Shell, Mitsui et Mitsubishi, à qui elle fournit conseils et recommandations sur la façon de minimiser les risques des développements gaziers et pétroliers pour les baleines et certaines portions au moins de leurs habitats. Une part majeure de ce travail fut la création, en 2006, du Comité consultatif sur la baleine grise occidentale (WGWAP), un panel de scientifiques indépendants qui fournit des conseils scientifiques pour les plans opérationnels de la compagnie. Ce panel fut un partenariat réussi qui a convaincu la compagnie de détourner le tracé de pipelines sousmarins pour éviter les sites d'alimentation des baleines, sur les conseils de l'UICN. Plus récemment, les recommandations du WGWAP au sujet des activités sismiques qui dérangeaient les populations de baleines ont entraîné l'arrêt des études sismiques dans la région (Communiqué de presse de l’UICN du 24 avril 2009 - http:// www.iucn.org/about/work/programmes/marine/ marine_news/?3069/Stop-all-oil-and-gasactivities-that-could-harm-Western-Gray-Whalessays-panel).

\section{AVANTAGES D'UNE COLLABORATION CONSERVATION/ENTREPRISE}

La collaboration entre le secteur privé et les organisations de conservation peut encourager une conception novatrice et des solutions techniques aussi bien pour les entreprises que pour la conservation. Les organisations de conservation peuvent aider les sociétés à améliorer leurs performances opérationnelles au point de vue de l'environnement. Cela peut se faire par la conception, par biomimétisme, de 
produits ou de bâtiments, ou par des conseils en matière de réductions d'émissions, d'efficacité opérationnelle ou de sources d'énergie et de technologies alternatives. Le fait de créer une chaîne logistique plus verte (et plus efficiente) peut avoir de nombreux effets secondaires positifs, surtout pour les grandes entreprises. Une chaîne logistique durable transmet ses normes et ses critères environnementaux vers ses fournisseurs, dans la mesure où ils font partie des critères liés à leurs demandes d'offres et des normes exigées des fournisseurs de matières premières. Les normes environnementales permettent aux entreprises de forger des relations plus solides avec leurs fournisseurs, avec un bénéfice pour la santé, la sécurité et la qualité. Si l'on intègre des considérations pour la conservation très tôt dans la conception du projet ou du site, cela paiera à long terme.

D'autre part, les organisations de conservation peuvent profiter des conseils du secteur privé pour la gestion de projets, les relations publiques, la gestion financière et la conception d'opérations complexes. Pour les organisations de conservation, le défi, en termes de chaîne logistique, consiste à déterminer où leurs conseils peuvent être les plus utiles en matière de conservation, jusqu'où dans la chaîne les normes de conservation peuvent se transmettre de sous-contractant à sous-contractant, et enfin jusqu'à quel niveau de la chaîne logistique une société peut être tenue pour responsable.

\section{DÉFIS FUTURS}

Lorsqu'une société a décidé qu'elle va rendre ses opérations plus vertes, quels changements opérationnels sont nécessaires et comment une organisation de conservation peut-elle contribuer au mieux ? Comment réconcilier les intérêts privés avec le bien de tous?

La sphère environnementale reconnaît généralement qu'une responsabilité sociale et environnementale d'entreprise et des réglementations gouvernementales plus strictes sont nécessaires, tout comme c'est le cas dans les marchés financiers mondiaux. La question est de savoir quels genres d'initiatives ou de réglementations volontaires pourront assurer des résultats qui répondent aux exigences duales de la conservation et de la profitabilité.

De nombreuses sociétés sont intéressées par un partenariat avec des organisations de conservation pour avoir de meilleurs impacts environnementaux ou pour faire des économies. Mais peu s'intéressent vraiment à apporter des changements fondamentaux et, éventuellement, perturbateurs dans leurs pratiques de gestion.

Certaines compagnies veulent entamer des partenariats avec des organisations environnementales pour améliorer leurs pratiques, mais la plupart des discussions tournent plus autour de pratiques améliorées que de pratiques nouvelles.

Les partenariats entre des organisations de conservation et le secteur privé peuvent aider à rendre les affaires plus vertes. Mais tant que les entreprises vertes ne deviennent pas la norme et que la compétition entre sociétés, basée sur des critères verts, ne forcera pas les entreprises à apporter des changements profonds et fondamentaux, le nombre d'entreprises réellement « vertes » restera modeste. Pour encourager la transition vers des " entreprises vertes ", les organisations de conservation et le secteur privé devront développer un langage commun pour évaluer les nombreux rôles que la biodiversité peut jouer dans le monde des affaires. A terme, celui-ci devra reconnaître cet aspect de plus en plus et c'est en son sein qu'un leadership devra se créer. Pendant ce temps, il faudra aussi un meilleur dialogue entre industries, société civile et organismes de contrôle. 
De tous les écosystèmes terrestres, ce sont les forêts qui contiennent le plus d'espèces, et 75\% des Centres de diversité botanique se trouvent dans des forêts. D'après l'Organisation des Nations Unies pour l'alimentation et l'agriculture (FAO), le couvert forestier augmente très légèrement (3,86 milliards d'ha en 2000 et 3,95 milliards en 2005), mais ces chiffres comprennent les plantations et la régénération des forêts tempérées. Si l'on exclut les plantations, le rythme de la déforestation s'est maintenu à près de 13 millions d'ha par an entre 1990 et 2005, avec quelques signes d'une diminution significative avec le temps (FAO, 2005). Cependant, au niveau des espèces proprement dites, la Liste rouge 2008 de l'UICN des espèces menacées rapporte que 172 des 620 espèces (28\%) de conifères sont menacées d'extinction (UICN, 2008d).

L'État des forêts du monde 2009 (FAO, 2009a) dresse un tableau très divers des problèmes de forêts dans le monde. Si l'on prévoit que la superficie des forêts va se stabiliser dans les pays développés d'Asie, en Amérique du Nord et dans certaines parties de l'Asie de l'Ouest et centrale, on s'attend aussi à ce qu'elle diminue dans la plus grande partie de l'Afrique et en Amérique du Sud encore que, pour cette dernière, les forêts plantées devraient augmenter. Le fait de sensibiliser les gens à la grande variété des services fournis par les forêts, et particulièrement les services relatifs à l'atténuation et à l'adaptation aux changements climatiques, permet d'attirer un nouveau public et, éventuellement, de nouveaux investissements dans la gestion durable des forêts. Le rapport note des innovations constantes dans le secteur forestier, mais aussi un fossé entre pays développés et pays en développement pour l'accès à ces innovations. Enfin, le rapport se demande quels impacts la crise économique de 2008/2009 va avoir sur les forêts dans le monde entier et si le développement va emprunter à l'avenir une " voie verte " qui favorisera la gestion durable des forêts.

Ce déclin mondial des forêts est un problème pour chacun, mais spécialement pour les pauvres des régions rurales. En 2004, Vedeld et al. notaient que les forêts procuraient aux familles rurales $22 \%$ de leurs revenus dans 17 pays répartis sur trois continents. La majorité des revenus proviennent de la nourriture trouvée dans la nature et du bois de feu, le fourrage, les grumes, les chaumes et les plantes médicinales occupant aussi une place importante. Les forêts et les nombreux services qu'elles offrent sont vraiment une part importante de la richesse des pauvres.

Aujourd'hui, la conservation des forêts se focalise sur la gestion à l'échelle du paysage, qui permet une meilleure application des lois et une meilleure 
gouvernance des forêts ; elle applique une approche par écosystème et encourage les dialogues et les partenariats qui mettent en évidence le rôle des forêts dans la subsistance des communautés locales. Chacun de ces thèmes figure en bonne place dans l'agenda des forêts et a reçu une attention bien méritée au CMN de Barcelone.

\section{DES FORÊTS FONCTIONNELLES GRÂCE À LA}

\section{RESTAURATION DU PAYSAGE FORESTIER}

Les forêts, avec leur riche biodiversité, fournissent un grand nombre de biens et de services qui contribuent au bien-être des hommes. Pourtant, ces services exigent une approche qui va au-delà des arbres et adopte une vision plus large de l'aménagement du territoire, et qui favorise en retour des bénéfices environnementaux, sociaux, culturels et économiques pour les populations.

Le monde de la conservation a largement adopté la gestion durable des forêts (GDF). Sayer et Maginnis (2005) ont proposés dix principes de bonne pratique pour la GDF (Encadré 16.1).

Les approches par écosystème, la GDF et la restauration des paysages forestiers ont évolué

\section{Encadré 16.1 Dix principes de bonne pratique pour une gestion durable des forêts}

1. Il n'existe pas une approche unique par écosystème ; elles sont au contraire multiples et doivent être adaptées et appliquées à chaque situation de manière pragmatique.

2. Les hommes font partie des écosystèmes - leurs emplois, leurs moyens de subsistance et la production de richesses sont aussi importants que les oiseaux et les singes.

3. Toute gestion environnementale doit être modulable : nous gérons, apprenons, adaptons et gérons de nouveau.

4. Les approches par écosystème requièrent des outils qui mesurent les performances de la totalité du système, dont les bénéfices environnementaux et les améliorations des moyens de subsistance des populations.

5. Des droits fonciers clairs et défendables, des institutions démocratiques et le règne du droit sont des éléments importants d'un environnement propice à des approches par écosystème.

6. Les forestiers professionnels doivent être éclectiques, avoir d'excellentes compétences interpersonnelles et gagner le respect de tous les groupes qui sont parties prenantes.
7. La science ne donne pas les réponses, mais elle nous aide à apprendre de nos erreurs, à nous adapter et à explorer des options novatrices.

8. Le côté souple des approches par écosystème est plus important que le côté rigide. Ces approches ne sont pas qu'une formule - elles impliquent de nouvelles attitudes, de nouvelles approches, de nouvelles compétences et une gamme de capacités plus étendue.

9. De nombreux éléments des approches par écosystème ne relèvent pas directement du contrôle des Départements des forêts ; ces agences doivent donc apprendre à user de leur influence et à négocier des arrangements avec les autres parties prenantes.

10.Les approches par écosystème ne feront pas disparaître les conflits ; elles peuvent rendre les compromis plus explicites mais il y aura toujours des gagnants et des perdants. Les approches par écosystème peuvent aider à réduire les différentiels de pouvoir entre les parties prenantes et mener à des résultats plus équitables, garantissant ainsi que la société en général et les groupes de parties prenantes en particulier gagnent plus et perdent moins

Source : Sayer et Maginnis, 2005 
et vont désormais au-delà des caractéristiques biophysiques pour inclure des composantes sociales, politiques et autres du système (Sayer et al., 2007). La restauration des paysages forestiers se fait à une échelle qui intègre tous les types paysagers environnants, créant une mosaïque de forêts, de terres agricoles, d'aires protégées et d'installations humaines où ont lieu la planification et la réalisation, et qui incorpore aussi la participation de toutes les parties prenantes concernées en fonction des utilisations multiples de ce paysage qui sous-tendent les moyens de subsistance (Fisher et al., 2008).

Le concept unificateur de ces approches tient à l'idée d'intégrer conservation et développement. Les conservationnistes ont souvent la lourde tâche de convaincre les communautés locales que les bénéfices à long terme de la conservation vont dépasser les bénéfices à court terme des récoltes en forêt. Sayer et al. (2007) proposent de planifier des projets qui incluent des indicateurs fondés sur les cinq atouts majeurs (capital financier, social, physique, humain et naturel) comme moyens de s'assurer que les perspectives des populations locales et de la conservation sont réunies.

L'intégration des connaissances traditionnelles dans la conception et la mise en œuvre des projets est cruciale pour préserver à court et à moyen terme l'intérêt pour les projets. Les conservationnistes doivent envisager très soigneusement les implications sociales et environnementales des projets, comme les droits fonciers, les besoins de bonne gouvernance, les droits des populations autochtones et la corruption. Le fait d'ignorer les causes originelles de la déforestation, qui sont souvent des causes sociales, fut une des raisons principales des échecs des premiers programmes de conservation des forêts.

Alors quelles sont les orientations futures des mesures de conservation dans le développement des paysages forestiers? Il y a d'abord les
Encadré 16.2 Forêt vs agriculture - le cas de la Réserve forestière de Mabira

La Réserve forestière de Mabira, sur les rives du lac Victoria, en Ouganda, abrite une faune sauvage de valeur, sert de ressource en grumes, procure des services écosystémiques liés à l'équilibre hydrique, et les forêts pluviales sont une destination touristique. Suite au projet d'éclaircir un tiers de la réserve pour l'agriculture, des chercheurs locaux ont calculé les valeurs de la forêt. Cette évaluation économique de la forêt montre que, vu à court terme, faire pousser de la canne à sucre générerait plus de bénéfices économiques que de conserver la réserve forestière, avec un rendement de 3,6 millions de dollars par an, par rapport à 1,1 million de dollar pour la conservation. Mais la production de canne à sucre n'est optimale que pendant une courte période d'environ cinq ans. Lorsque l'on compare les alternatives d'aménagement du territoire sur la durée du stock de grumes, 60 ans, les avantages de la forêt et des services écosystémiques qu'elle offre dépassent ceux de la plantation de canne à sucre.

Source : Environment Times \#5, http://werere.grida.no/ publications/et/ep5/page/2351.aspx

nouvelles opportunités et menaces suscitées par l'expansion agricole et agro-industrielle et par l'instabilité économique. De nombreuses études récentes ont cherché à identifier le seuil à partir duquel il est plus profitable pour les occupants des forêts de nettoyer le terrain pour l'agriculture que de garder la forêt sur leurs terres (Encadré 16.2). Les conditions qui pousseraient à cette déforestation seraient par exemple une hausse des prix mondiaux de produits alimentaires comme le soja ou le bœuf, ou encore une meilleure accessibilité des forêts grâce à l'amélioration des infrastructures des transports. En plus de 
l'évaluation des coûts/bénéfices sur des périodes suffisamment longues, d'autres techniques peuvent encourager la GDF, comme les systèmes de certification, les coupes de grumes à impact réduit et des instruments financiers comme le paiement pour les services écosystémiques (PES).

\section{MISE EN APPLICATION DES LOIS ET GOUVERNANCE EN FORÊT}

L'échec des mécanismes de bonne gouvernance pour les ressources forestières est au cœur des problèmes relatifs à la GDF. La Banque mondiale (2006) estime que les pertes des gouvernements dues aux coupes illégales de bois se montent à 10 milliards de USD par an, bien plus que le montant de l'Aide publique au développement (ODA) investi dans la GDF. De plus, on estime que 5 milliards de dollars sont perdus chaque année à cause de la corruption, en royalties non récoltées et en taxes non perçues sur des récoltes illégales sanctionnées par la loi. En fin de compte, ce sont les millions de personnes dont les moyens de subsistance dépendent des ressources forestières qui sont les grands perdants.

Au Congrès mondial de la nature $(\mathrm{CMN})$ d'Amman, en Jordanie, en 2000, les Membres de l'UICN ont reconnu l'impact de la corruption sur le secteur forestier et la nécessité d'encourager une meilleure gouvernance (Résolution CMN 2.039) (UICN, 2000a). De nombreux pays et régions essaient aujourd'hui de traiter ce problème des crimes contre les forêts et de leurs conséquences en s'engageant dans l'Application des réglementations forestières et la gouvernance (FLEG).

Le Programme d'action du G8 pour les forêts (1998) a identifié les coupes illégales comme un obstacle majeur à la gestion durable des forêts. Le Programme a fourni un incitant important pour multiplier les actions contre les coupes illégales. Par la suite, trois Conférences ministérielles sur l'Application des réglementations forestières et la gouvernance (FLEG), à savoir la FLEG d'Asie de l'Est (EA FLEG) en septembre 2001, à Bali, en Indonésie ; la FLEG d'Afrique à Yaoundé, au Cameroun, en octobre 2003 ; et la FLEG Europe et Asie du Nord à Saint Petersburg, Fédération de Russie, en novembre 2005. Les trois Conférences FLEG ont rassemblé des gouvernements, des industries, des ONG et des chercheurs venant de pays situés dans et en dehors de la région pour améliorer la gouvernance et susciter un dialogue international sur les activités illégales dans le secteur forestier. Ils ont également collaboré pour établir des cadres qui permettraient aux gouvernements des pays producteurs de travailler les uns avec les autres pour améliorer les liens et harmoniser les réglementations, et avec les gouvernements des pays consommateurs pour s'attaquer aux coupes de bois et aux pratiques commerciales illégales.

Une importante initiative est venue de la Commission européenne (CE) lorsqu'elle a approuvé un Plan d'action pour l'Application des réglementations forestières, gouvernance et échanges commerciaux (FLEGT) en 2003. Ce plan fut approuvé par les Etats membres la même année. Ce Plan d'action veut interdire l'entrée de toute grume illégale sur le marché de l'Union européenne (EU), par le biais de stratégies comme l'application d'accords de partenariats volontaires (APV) avec les pays producteurs. Ces accords instaureront dans chaque pays un système de licences pour les grumes légales qui auront l'autorisation d'être importées par les pays membres de l'UE, empêchant les produits ligneux dépourvus de licence et donc potentiellement illégaux d'entrer sur le marché de l'UE. En mai 2009, par exemple, un APV a été initié avec le Ghana, et les négociations avec le Congo (Brazzaville) et le Cameroun sont presque terminées. Des négociations sont aussi en cours avec la Malaisie et l'Indonésie, et d'autres vont commencer en 2009 avec le Gabon. La CE discute aussi avec la Chine, le Vietnam et d'autres pays pour traiter le problème des coupes de bois illégales par d'autres voies. 
Parmi les autres stratégies utilisées ou en discussion, citons les politiques de passations de marché et des législations supplémentaires pour empêcher les grumes illégales de pénétrer des marchés de consommateurs.

Les leçons tirées de ces initiatives comprennent :

- Les coupes de grumes illégales sont un résultat mais aussi un symptôme d'une mauvaise gouvernance forestière, et les mesures pour favoriser une meilleure application de la législation devront s'accompagner de réformes plus fondamentales de la gouvernance forestière pour corriger les causes sous-jacentes des coupes illégales.

- Les coupes "légales" ne font pas obligatoirement partie d'une gestion forestière " équitable et durable ». L'UICN veut mettre en place des accords de gouvernance forestière qui favorisent la gestion forestière durable et équitable.

- Les mesures pour traiter les coupes illégales et les réformes plus générales de la gouvernance forestière doivent se baser sur des processus efficaces qui englobent diverses parties prenantes afin d'améliorer la qualité des décisions et d'amplifier le support sociétal de leur application.

- Les processus efficaces multipartenaires qui accompagnent les initiatives visant à corriger les coupes illégales, comme les APV, pourraient devenir le tremplin nécessaire pour entamer des réformes plus profondes de la gouvernance forestière.

- Des expériences récentes de réformes de la gouvernance forestière, dans le contexte de la FLEG(T) et les processus multipartenaires associés fournissent des intrants très intéressants dans les discussions sur les accords en matière de gouvernance forestière qui doivent appuyer les programmes de Réduction des émissions issues de la déforestation et de la dégradation (REDD).
PARTENARIATS FONDÉS SUR LA FORÊT EN AIDE AUX MOYENS DE SUBSISTANCE

Les forêts fournissent de nombreux biens et services à de multiples parties prenantes. Cela doit se refléter dans la manière dont les paysages forestiers sont gérés et préservés, mais cela ne peut se faire équitablement que si l'on écoute toutes les voix concernées. Les partenariats sont donc indispensables pour partager les besoins et les points de vue et pour trouver des synergies. Dans ce contexte, une approche de la gouvernance et de la gestion forestière qui se ferait d'un unique point de vue serait contreproductive - si ceux qui ont un intérêt légitime dans les forêts n'ont aucun mot à dire sur l'avenir des paysages forestiers, il est probable qu'ils vont saboter toute tentative de faire des progrès. Supposer que les forêts sont destinées à « la séquestration du carbone » ou à « la conservation de la biodiversité " n'autorise pas pour autant à détourner l'attention des biens et services qu'elles procurent aux populations rurales, dont un nombre considérable comptent sur ces ressources pour leur subsistance élémentaire. Il ne suffit pas non plus de supposer que les scénarios gagnant-gagnant simplistes entre conservation et développement pourraient se produire de cette façon. Pour satisfaire de multiples voix en concurrence, il faut négocier des compensations, ce qui se fait plus facilement dans un esprit de collaboration et de partenariat.

Le Dialogue sur la forêt tropicale, dont le secrétariat se trouve à l'Université de Yale, encourage les dialogues depuis sa création en 1998. Il part du principe qu'il faut susciter la confiance entre les différents groupes qui participent aux dialogues et qu'il faut leur donner des outils, des idées et un environnement dans lequel ils peuvent former leurs propres partenariats. Ces partenariats ne doivent pas forcément aboutir à des compromis inopérants ; la création même d'un consensus peut être radicale. L'UICN a contribué à Intensively Managed Planted Forests: towards best practice (Forêts plantées gérées intensivement : vers de meilleures 
pratiques) et Au-delà de REDD: le rôle des forêts dans le changement climatique, une déclaration sur les forêts et les changements climatiques fondée sur un consensus.

Le Partenariat de collaboration sur les forêts (CPF) est un accord volontaire de 14 organisations et secrétariats internationaux qui ont d'importants programmes pour les forêts (CIFOR, FAO, OIBT, IUFRO, CDB, FEM, CCD, FNUF, CCNUCC, PNUD, PNUE, ICRAF, BM, UICN) ${ }^{3}$. La mission $\mathrm{du}$ CPF est de favoriser la gestion, la conservation et le développement durable de tous les types de forêt et de consolider les engagements politiques à long terme allant dans ce sens. Les membres du CPF travaillent de plus en plus ensemble dans des projets et mobilisent des ressources qui aident les pays à atteindre leurs objectifs en matière de forêts et à mettre en œuvre une gestion durable des forêts (FAO, 2009b).

En 2007, la Banque mondiale a proposé de créer une initiative de Partenariats forestiers en développement (PFD) qui lie les procédures locales et mondiales et favorise le fait que la prise de décisions sur la scène internationale reflète les vues et les besoins des parties prenantes forestières. La Banque mondiale a alors demandé à l'Institut international pour l'environnement et le développement (IIED) de réaliser une évaluation indépendante de sa proposition avec une grande variété de parties prenantes. Plus de 600 experts forestiers ont répondu à l'enquête de l'IIED, ou y ont participé dans des groupes focaux au Brésil, en Chine, au Ghana, en Guyane, en Inde, et Russie et au Mozambique, ainsi que lors de réunions internationales. L'UICN a aussi joué un rôle clé dans les PFD. Cette initiative, soutenue par la FAO et la Banque mondiale, veut rendre la foresterie véritablement durable en construisant et en renforçant de nouveaux partenariats qui reflètent les besoins locaux et protègent les biens publics dans le monde entier. Par sa focalisation spécifique sur le fait d'être pilotée de bas en haut, elle differe totalement du CPF et elle le complète. Le point focal immédiat du PFD de l'UICN est de développer des partenariats au Mozambique, au Ghana et au Guatemala.

Certaines ONG ont fait un intéressant pas en avant en aidant des communautés locales à renforcer leurs capacités en développement d'entreprises. Forest Trends, un Membre de l'UICN, a mis au point un Business Development Facility, un mécanisme de développement d'entreprises pour apporter une assistance technique aux opérateurs forestiers en évaluant, identifiant et développant des opportunités pour que les sources de revenus de produits non ligneux optimisent la valeur de la forêt, comme la séquestration de carbone, la conservation des bassins hydrologiques et la conservation de la biodiversité. Les conservationnistes peuvent aider les gens qui vivent en forêt en passant d'une " approche par un seul atout » où la coupe de grumes est considérée comme la seule valeur réelle des forêts, à une « approche par atouts multiples » qui diversifie les opportunités d'améliorer les moyens de subsistance en capitalisant les produits non ligneux et les services et en facilitant l'accès aux marchés.

\section{FORÊTS ET CHANGEMENTS CLIMATIQUES}

Le chapitre 5 souligne le rôle des forêts dans l'atténuation des changements climatiques par la REDD. En plus de la REDD, les forêts présentent une autre possibilité dans ce domaine. Les terres forestières dégradées

\footnotetext{
${ }^{3}$ Intitulés complets de ces sigles : CIFOR - Centre international de recherche sur les forêts; FAO : Organisation des Nations Unies pour l'alimentation et l'agriculture ; OIBT : Organisation internationale des bois tropicaux; IUFRO : Union internationale des instituts de recherches forestières ; CDB : Convention sur le diversité biologique ; FEM : Fonds pour l'environnement mondial ; CCD : Convention des Nations Unies sur la lutte contre la désertification dans les pays gravement touchés par la sécheresse et/ou la désertification, en particulier en Afrique; FNUF : Forum des Nations Unies sur les forêts; CCNUCC : Convention-cadre des Nations Unies sur les changements climatiques ; PNUD : Programme des Nations Unies pour le développement; ICRAF : Conseil international de recherches en agroforesterie ; BM:Banque mondiale.
} 
couvrent aujourd'hui quelque 800 millions

d'hectares. Même si leurs stocks de carbone sont sensiblement réduits, ces terres conservent encore un couvert forestier suffisant pour qu'elles ne soient pas classées comme éclaircies complètes et donc pour être éligibles pour des fonds de reboisements dans le cadre des mécanismes de Kyoto. Le Quatrième rapport d'évaluation du Groupe d'experts intergouvernemental sur l'évolution du climat (GIEC) estime que la restauration de ces sols pourrait représenter approximativement 117 millions de tonnes d'équivalent carbone (en " traduisant » les autres gaz à effet de serre en dioxyde de carbone) d'ici 2030. Cela équivaut à une fois et demi le potentiel estimé disponible pour prévenir la déforestation jusqu'en 2030. La restauration des sols forestiers dégradés présente un triple bénéfice : des émissions évitées en stoppant la dégradation en cours ; une quantité significative de carbone supplémentaire séquestré grâce à la restauration ; et les avantages de l'adaptation aux changements climatiques à l'échelle du paysage, en ce qui concerne la fourniture de services par un écosystème restauré, comme une meilleure régulation des cycles hydrologiques.

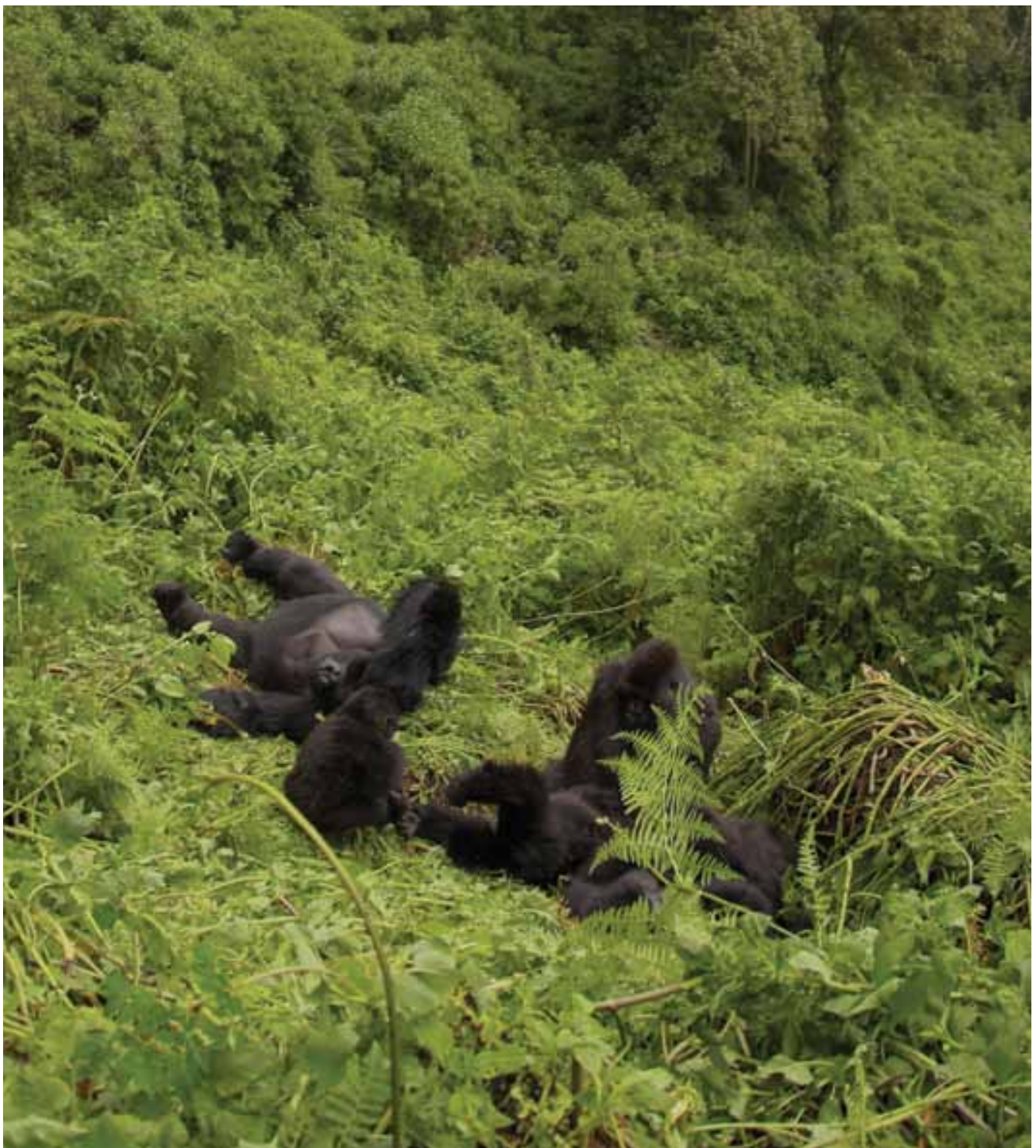




\section{Systèmes marins : \\ 1. orienter la conservation vers la mer}

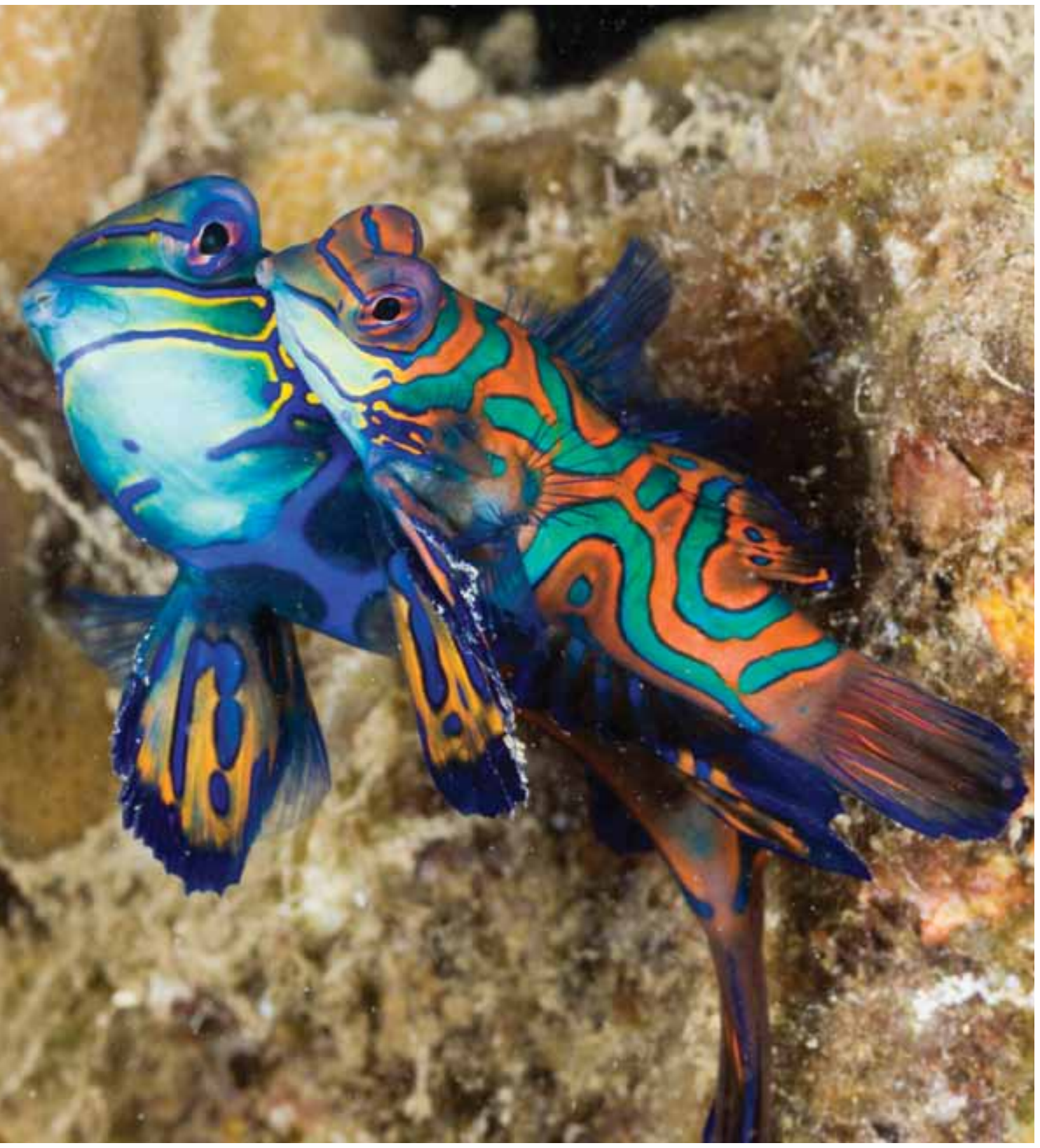


Après être resté dans l'ombre pendant de nombreuses années, alors qu'il représente la grande majorité de la surface de la planète, le domaine marin requiert aujourd'hui notre attention. Comme les ressources terrestres s'épuisent de plus en plus, le monde se tourne vers les mers pour y trouver des solutions. Et comme les solutions terrestres aux problèmes des changements climatiques sont aussi de plus en plus difficiles à trouver, les politiques se demandent comment les océans pourraient les y aider.

Parmi les nombreux problèmes à traiter d'urgence dans les milieux marins, citons la concentration croissante du $\mathrm{CO}_{2}$ et d'autres gaz à effet de serre (GES) et la surpêche. Dans cette lutte, deux outils majeurs, qui doivent être affinés et adaptés aux océans, sont la gouvernance des ressources marines dans les mers situées au-delà de toute juridiction nationale et la planification spatiale, comme les aires marines protégées (AMP).

\section{OCÉANS ET CHANGEMENTS CLIMATIQUES}

Les océans jouent un rôle crucial dans la régulation du climat mondial et ils fournissent aussi nourriture et revenus à des milliards de personnes de par le monde. Comme le climat mondial change à un rythme sans précédent, principalement à cause des émissions de GES d'origine humaine, il n'est plus possible d'ignorer les preuves des impacts sur les environnements marins et côtiers. Le réchauffement des océans, l'augmentation de l'acidité des eaux, le blanchissement des coraux et la hausse du niveau des mers sont des effets déjà visibles et ils ont des conséquences sérieuses sur la biodiversité marine et sur les sociétés humaines. La communauté internationale a donc comme obligation prioritaire de mieux comprendre les changements climatiques et la façon dont ils interagissent, exacerbent de nombreux stress directs comme la pollution et la surpêche, et d'employer ces connaissances pour atténuer les changements climatiques ou pour s'y adapter.

Les océans seront à la fois les victimes et la solution possible des impacts des changements climatiques. Les récifs coralliens sont un des écosystèmes les plus fragiles du monde face aux changements climatiques, au point qu'on peut les considérer comme les « canaris dans la mine de charbon des changements climatiques ». Le rapport 2008 sur le statut des récifs coralliens du monde indique que $19 \%$ des récifs ont disparu, avec un impact sur 500 millions de personnes dont les moyens de subsistance dépendent de ces récifs (Wilkinson, 2008). Mais il n'y a pas que les récifs qui présentent des signes d'impacts des changements climatiques ; les mangroves 
et les autres écosystèmes côtiers sont aussi vulnérables à la hausse du niveau des mers. De plus hautes températures et des changements du taux d'acidité affectent les prairies sous-marines utilisées par la médecine traditionnelle, pour la fabrication de meubles et de toits pour les maisons, qui offrent aussi des habitats essentiels pour de nombreuses espèces, des poissons aux dugongs.

La pêche, et les communautés qui en vivent, ressentiront certainement les impacts des changements climatiques. Alison et al. (2009) ont comparé la vulnérabilité de 132 économies nationales face aux impacts possibles des changements climatiques sur les prises, et ils ont déterminé que le Malawi, la Guinée, le Sénégal, le Pérou, la Colombie, le Bangladesh, le Cambodge, le Pakistan et le Yémen étaient les pays les plus fragiles. Cette vulnérabilité est due aux effets combinés du réchauffement annoncé, de l'importance relative de la pêche dans les économies et des habitudes alimentaires nationales, et à la capacité sociétale limitée de s'adapter aux impacts et aux opportunités possibles.

Parmi les solutions aquatiques aux menaces des changements climatiques, une attire particulièrement l'attention, c'est le géo-engineering des océans. Le phytoplancton peut faire des océans le plus grand puits de carbone du monde; des propositions pour " géo-ingénieurer » les océans suggèrent d'augmenter l'absorption de carbone en stimulant la prolifération des algues par l'addition de fer et d'autres nutriments pour capter le $\mathrm{CO}_{2}$, ou d'injecter directement le $\mathrm{CO}_{2}$ dans des structures géologiques sous le fond marin, processus connu sous le nom de capture et stockage de carbone (CSC) (Victor et al., 2009). Les nombreuses discussions enflammées sur le potentiel des océans pour aider à la mitigation des changements climatiques ne peuvent pas faire oublier le fait que nous en savons encore très peu sur les processus biochimiques océaniques et sur la capacité d'absorber le $\mathrm{CO}_{2}$. Si certaines études montrent que la fertilisation des océans ne stimule pas la prolifération des algues, il n'est toujours pas certain que le transport de carbone dans les sédiments des océans soit efficace, et les impacts de ces activités sur l'environnement marin, du plancton aux baleines, et sur le climat mondial restent inconnus et imprévisibles. Avant d'envisager la vente de compensations carbone pour des projets de fertilisation, il faut faire de nouvelles recherches pour en évaluer les risques pour nos océans ; les activités de fertilisation à grande échelle exigent la plus extrême prudence. De même, l'énergie et la rentabilité des CSC ne sont pas prouvées, et les implications de fuites éventuelles sont graves. Enfin, les Cadres réglementaires pour la fertilisation des océans et les CSC ne sont pas suffisants actuellement, et aucune des ces activités ne contribue pour le moment à réduire la production du $\mathrm{CO}_{2}$ d'origine humaine.

\section{SURPÊCHE}

\section{La Situation mondiale des pêches et de l'aquaculture} de l'Organisation des Nations Unies pour l'alimentation et l'agriculture (FAO, 2008a) répète les conclusions de son rapport de 2007 mais, si le fait que la situation soit restée stable au cours des deux dernières années est un signe positif, il n'en reste pas moins que la majorité des pêches dans le monde sont déjà totalement exploitées et que $28 \%$ de plus sont surexploitées. Le rapport confirme qu'en 2006, la pêche et l'aquaculture ont fourni plus de poissons de consommation que jamais et qu'une proportion croissante de ces poissons proviennent de l'aquaculture. Les tentatives pour contrer les principaux facteurs de la surpêche ont eu des résultats minimes. Ces facteurs sont la gestion des prises accessoires, la surcapitalisation des flottilles de pêches du monde entier, le contrôle de la pêche illégale et l'atténuation des dommages causés par certaines 
méthodes de pêche comme la pêche au chalut et celle au cyanure.

Le rapport de la FAO ne mentionne pas certaines autres observations inquiétantes constatées récemment. Quelque 26\% des requins et des raies du nord-est de l'Atlantique sont menacés d'extinction par la pêche excessive de ces espèces qui grandissent lentement (Gibson et al., 2008). De nombreux oiseaux de mer, en particulier les albatros, sont aussi menacés par les activités de pêche et ils peuvent faire partie des prises accessoires, encore que des changements apportés aux méthodes de pêche au thon par les longues lignes à Hawaï aient permis une réduction de $67 \%$ des prises accessoires d'oiseaux de mer (Gilman et al., 2008). Une autre conséquence troublante de nombreuses années de surpêche, spécialement pour des poissons comme le cabillaud, c'est le fait que certaines populations de poissons, au terme d'une décennie qui les a vues décliner dramatiquement, arrivent maintenant à maturité plus tôt et à une taille plus petite (Fudge et Rose, 2008). Les pratiques de pêche modernes aboutissent à des changements évolutifs bien plus rapides que nous n'aurions pu l'imaginer.

La volonté politique de gérer les pêches semble peu répandue. Un des événements récents les plus décevants fut la décision prise en novembre 2008 par la Commission internationale pour la conservation des thonidés de l'Atlantique (CICTA) d'adopter pour le thon de l'Atlantiquenord un quota supérieur de presque $50 \%$ au niveau de pêche recommandé, qui était pourtant proposé par ses propres conseillers scientifiques (UICN, 2008c). La population de thons rouges a atteint un niveau critique et tous les avis scientifiques se rejoignent sur la nécessité de réduire drastiquement le niveau de cette pêche, voire de la fermer pendant la saison de frai pour permettre aux stocks de se reconstituer. Les objectifs à court terme en matière d'économie et d'emplois prévalent sur les besoins à plus long terme des écosystèmes qui sont à la base de ces mêmes objectifs. Cela remet en question le rôle des organisations régionales de gestion des pêches et leur capacité de gérer des récoltes soutenables des ressources de haute mer. À plus petite échelle, cependant, on rapporte certains succès de gestion de pêches grâce à l'allocation de « catch shares ", des quotas partagés entre pêcheurs privés, une approche de la gestion des ressources essentiellement basée sur les droits (Costello et al., 2008).

Comme s'il ne suffisait pas de devoir traiter les impacts des décisions de gestion peu judicieuses, de nouveaux résultats sont maintenant disponibles concernant des glissements de distribution et des changements d'abondance de poissons et d'invertébrés d'intérêt commercial induits par les changements climatiques. Ils laissent penser que les espèces des eaux plus chaudes se déplacent vers de plus hautes latitudes et que la productivité de poisson risque de décliner aux basses latitudes (c.-à-d. dans la plupart des océans, des mers et des lacs tropicaux et subtropicaux) (FAO, 2008b). Alors que ces changements écologiques peuvent avoir des effets positifs dans les pays du nord, ils vont évidemment réduire les possibilités de captures dans les pays tropicaux. De plus, on prévoit que les océans tropicaux et polaires, tout comme les mers semi fermées, seront plus vulnérables face à l'invasion d'espèces non natives et au glissement de la distribution des espèces. Étant donné leur forte dépendance vis-à-vis de la pêche et de leur capacité réduite de s'adapter aux changements climatiques, les pays côtiers pauvres des tropiques, spécialement en Afrique, en Asie et dans le nord-ouest de l'Amérique latine, seront particulièrement affectés par les impacts des changements climatiques sur la pêche. Il faut apporter des réponses en matière de gestion, mais elles doivent être très soigneusement étudiées pour équilibrer les compensations entre gestion 
des pêches, conservation de la biodiversité et gestion d'autres activités maritimes humaines, pour le bien de tous à long terme.

\section{GOUVERNER LÀ OÙ AUCUN GOUVERNEMENT}

\section{NE GOUVERNE}

Les océans du monde entier doivent être vus dans leur ensemble, et la seule voie raisonnable vers l'avenir - qui constitue un fameux défi - doit être de jeter des ponts entre les multiples parties prenantes de l'océan et de ses ressources. Sans coordination, les régimes de gouvernance et de gestion centrés par secteurs ne conviennent pas pour traiter correctement les multiples menaces qui pèsent sur les environnements marins, ni pour évaluer les effets cumulés des activités ou l'impact d'une activité sur une autre. Ils ne conviennent pas non plus pour s'occuper du problème particulièrement épineux de la gouvernance au-delà de la juridiction nationale, à savoir en haute mer.

Néanmoins, les problèmes des mers reçoivent une attention politique importante chaque année aux Nations Unies, ce qui représente une occasion tangible et rapide d'obtenir des résultats en matière de politique environnementale.

L’Assemblée générale des Nations Unies s'occupe de la pêche illicite, non déclarée et non réglementée (IUU), des chaluts traînants, de la conservation des montagnes sous-marines et des autres écosystèmes marins fragiles, des ressources génétiques des hauts-fonds marins, et de mesures de gestion, par région, en dehors des juridictions nationales. La Convention sur la diversité biologique (CDB) de son côté traite des questions de conservation marine dans les régions sous juridiction nationale, y compris dans les AMP, et elle fournit des conseils scientifiques et techniques pour les AMP situées au-delà des juridictions nationales, comme par exemple l'identification des zones écologiquement et biologiquement importantes et la conception de réseaux d'AMP représentatifs.
Encadré 17.1 Dix principes de bonne gouvernance en haute mer

1. Liberté d'action conditionnelle en haute mer

2. Protection et préservation de l'environnement marin

3. Coopération internationale

4. Approche scientifique de la gestion

5. Accès public à l'information

6. Processus décisionnels transparents et ouverts

7. Principe de précaution

8. Approches par écosystème

9. Utilisation durable et équitable

10. Responsabilité des États, gardiens de l'environnement marin mondial

Source: http://cmsdata.iucn.org/downloads/10_principles_ for_high_seas_governance_final.pdf

L'Organisation mondiale du commerce (OMC), quant à elle, négocie une réforme des subsides accordés à la pêche, principalement pour répondre aux inquiétudes concernant l'exploitation non durable et les impacts environnementaux (ex. les prises accessoires). Dans un avenir prévisible, ces questions resteront importantes au point de vue politique et technique. La Convention de Londres (LC) a développé des réglementations au sujet de la séquestration de $\mathrm{CO}_{2}$ sous les fonds marins et met au point un Cadre d'évaluation pour les activités de recherches scientifiques concernant la fertilisation des océans. Les Parties à la LC ont appelé les Etats à s'abstenir de toute activité de fertilisation des océans et ont rédigé une résolution déclarant que les activités de fertilisation des océans autres que « les recherches scientifiques légitimes » sont contraires aux buts de la Convention de Londres et de son Protocole, 
n'ont actuellement droit à aucune exemption et ne doivent pas être autorisées.

Pour susciter une discussion internationale au sujet de cette réforme, 10 Principes de bonne gouvernance en haute mer ont été lancés au Congrès mondial de la nature à Barcelone, qui reflètent les principes fondamentaux que les pays ont adoptés dans divers traités et déclarations, mais qu'ils ont largement échoué à mettre en œuvre dans les quelque 50\% de la surface de la planète qui se trouvent en dehors de toute juridiction nationale (Encadré 17.1). Ces approches sont destinées à stimuler les progrès en identifiant des lignes directrices communes pour des actions à entreprendre.

\section{AIRES MARINES PROTÉGÉES (AMP)}

$\mathrm{Si}$, sur la terre ferme, la couverture totale des aires protégées représente environ $12 \%$, en mer, la couverture est de moins de $1 \%$ - un piètre pourcentage si l'on considère que $71 \%$ de la planète sont des océans. Les aires marines protégées, lorsqu'elles sont effectivement désignées, gérées et appliquées, peuvent fournir de nombreux avantages écologiques et socioéconomiques et aussi participer à la résilience des écosystèmes marins face aux pressions mondiales croissantes, et spécialement les changements climatiques. Pourtant, au rythme actuel, l'objectif mondialement accepté de protéger 10\% des océans du monde entier d'ici 2010 ne sera pas atteint avant 2060 - date à laquelle de nombreuses espèces marines, spécialement celles qui ont une valeur commerciale, pourraient bien avoir disparu.

La Commission mondiale des aires protégées (CMAP) de l'UICN va inclure un point focal sur l'amélioration des listes indicatives du patrimoine mondial pour des Sites marins et aussi sur le développement d'approches pour améliorer l'efficacité de gestion des sites marins existants.
Plusieurs évaluations mondiales ont conclu que les aires marines protégées bien gérées, fondées sur une connaissance approfondie du contexte local, peuvent être profitables pour les communautés locales tout en réalisant leurs objectifs de conservation. On a montré que les AMP efficaces aident à améliorer la productivité des pêches voisines, à augmenter et à diversifier les options économiques pour les populations locales, à favoriser la reconnaissance des droits de pêche traditionnels et ceux d'autres utilisateurs et à résoudre des conflits locaux. Les systèmes d'AMP jouent aussi un rôle important dans la résilience croissante des écosystèmes et ils peuvent favoriser l'adaptation aux changements climatiques.

Pour être efficaces, les systèmes d'aires marines protégées doivent être cohérents d'un point de vue écologique et ils doivent s'inscrire dans des Cadres intégrés de gestion des océans qui englobent toute la gamme des activités humaines et leurs impacts à l'intérieur et à l'extérieur des aires protégées. Un système efficace et représentatif exige que les informations spatiales et temporelles sur le milieu marin soient actualisées. Le lancement d'un calque AMP sur Google Earth, accessible pour des millions d'utilisateurs et aussi un portail internet pratique sur les AMP mondiales qui se trouve sur www.protectplanetocean.org devraient susciter beaucoup d'intérêt et favoriser le partage d'informations entre gouvernements, conservationnistes, praticiens et grand public en général, pour arriver aux meilleures connaissances possibles. Les décisions quant à l'emplacement de nouvelles AMP doivent être étayées par des informations pertinentes sur les espèces, les habitats et les moyens de subsistance dans tous les océans. De premiers efforts ont déjà permis de rassembler des informations sur des espèces et des données sur des habitats, pour une planification optimisée des AMP, preuve d'un 
nouveau partenariat novateur d'experts pour la conservation.

\section{L'AVENIR DE NOS MERS ET DE NOS OCÉANS}

Il est évident que des concentrations croissantes de $\mathrm{CO}_{2}$ et d'autres gaz à effet de serre (GES) et les changements causés au climat terrestre et à la chimie des océans constituent des risques significatifs pour les océans et les écosystèmes côtiers. Il faut traiter ces risques dans le contexte des nombreux catalyseurs directs des changements, dont la pollution et la surexploitation, qui sont pour la plupart exacerbés par les changements climatiques.

Alors que les menaces qui pèsent sur l'environnement marin sont à certains points de vue semblables à celles des habitats terrestres, il faut ancrer les solutions dans une bonne compréhension et une gestion correcte des différences entre travail en mer et sur terre. De plus, il faut d'urgence un accord mondial sur des stratégies de gestion dans les zones situées hors des juridictions nationales. Il faut aussi adopter des approches de la gestion des pêches basées sur les droits et atteindre les objectifs fixés en matière de systèmes d'AMP efficacement gérés et écologiquement cohérents. 


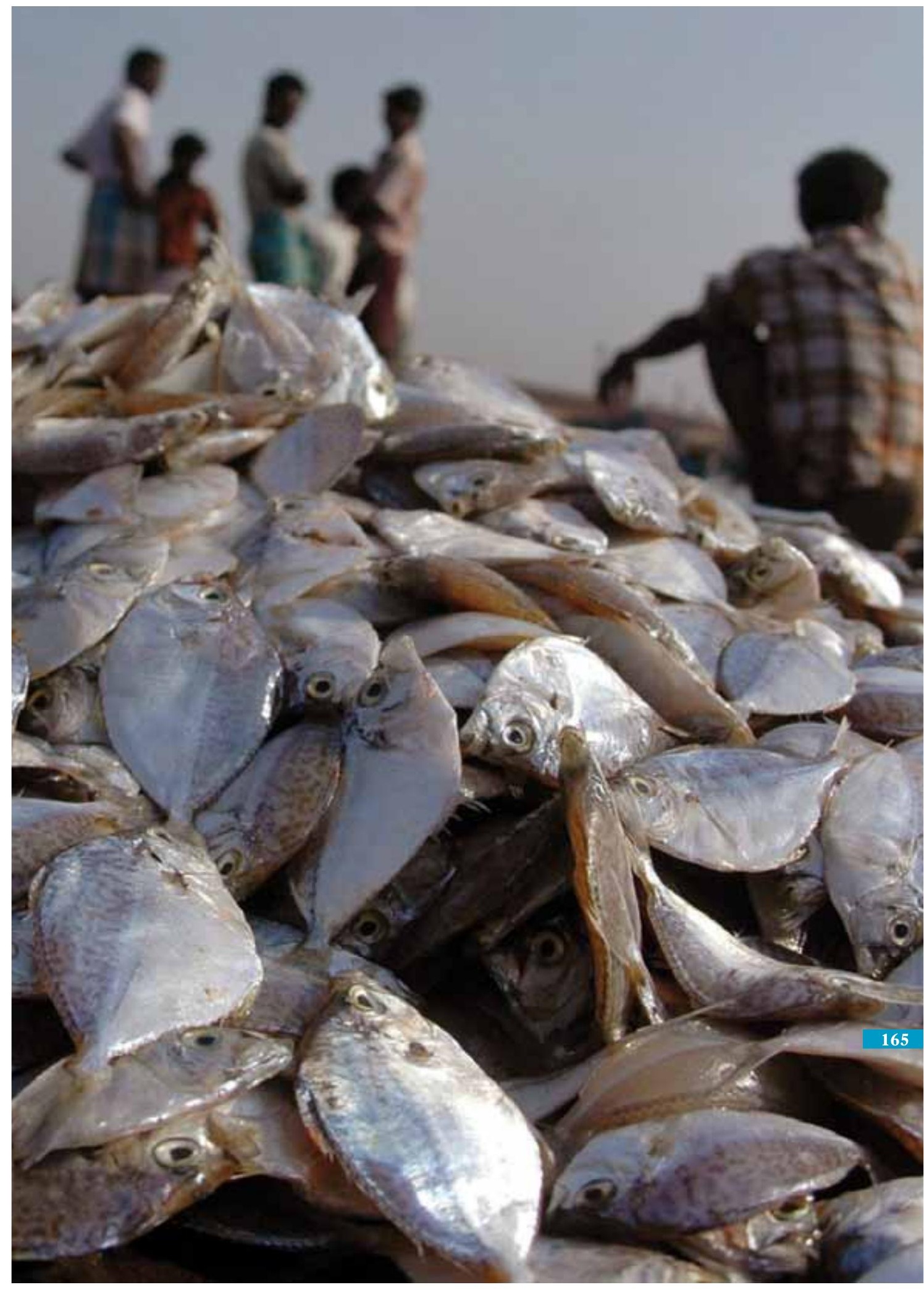


18. Systèmes arides : une question d'eau

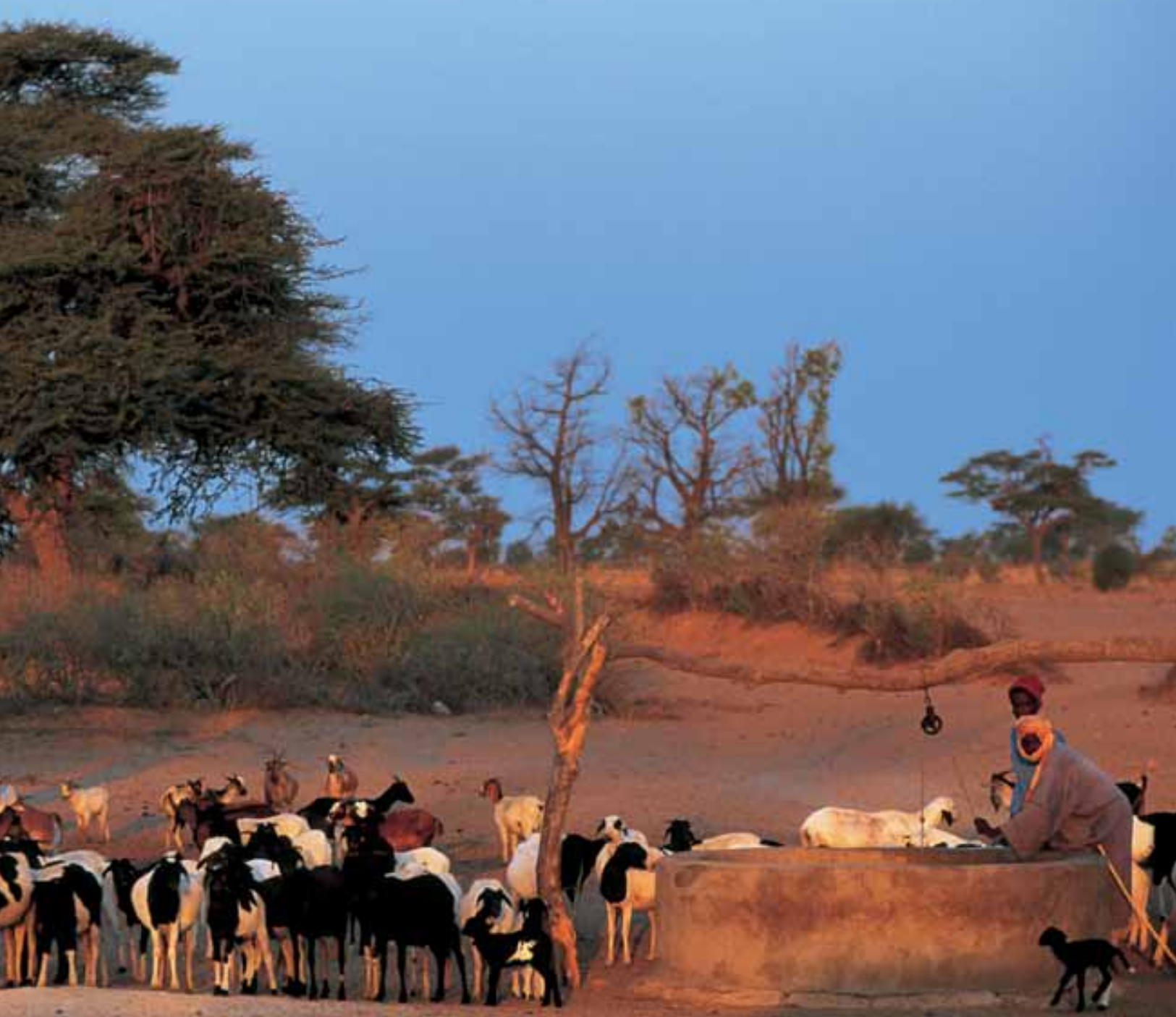


L'UICN considère les terres arides comme des régions et des paysages tropicaux tempérés dont l'indice d'aridité est de moins de 0,65, ce qui comprend les sous-types arides suivants : subhumide sec, semi-aride, aride et hyper aride (déserts) (UICN, 2008b). Ces systèmes arides couvrent approximativement $40 \%$ de la surface terrestre et peuvent se trouver aussi bien dans des pays en développement que développés. Au moins 30\% des plantes cultivées proviennent des régions arides qui accueillent aussi $47 \%$ des oiseaux endémiques et $26 \%$ des aires protégées du monde.

L'UICN a une approche très globale de la mosaïque des paysages arides : elle y inclut donc les zones urbaines et les zones humides comprises dans des régions et des paysages arides. Pourtant, pour les besoins des programmes de travail de l'UICN sur les terres arides, les zones arides de l'Arctique et de l'Antarctique ont été exclues parce que ce sont des endroits où c'est la température plus que la disponibilité en eau qui limite la productivité biologique. De plus, l'UICN inclut des zones arides saisonnières dans la gamme de ses travaux en terres arides,

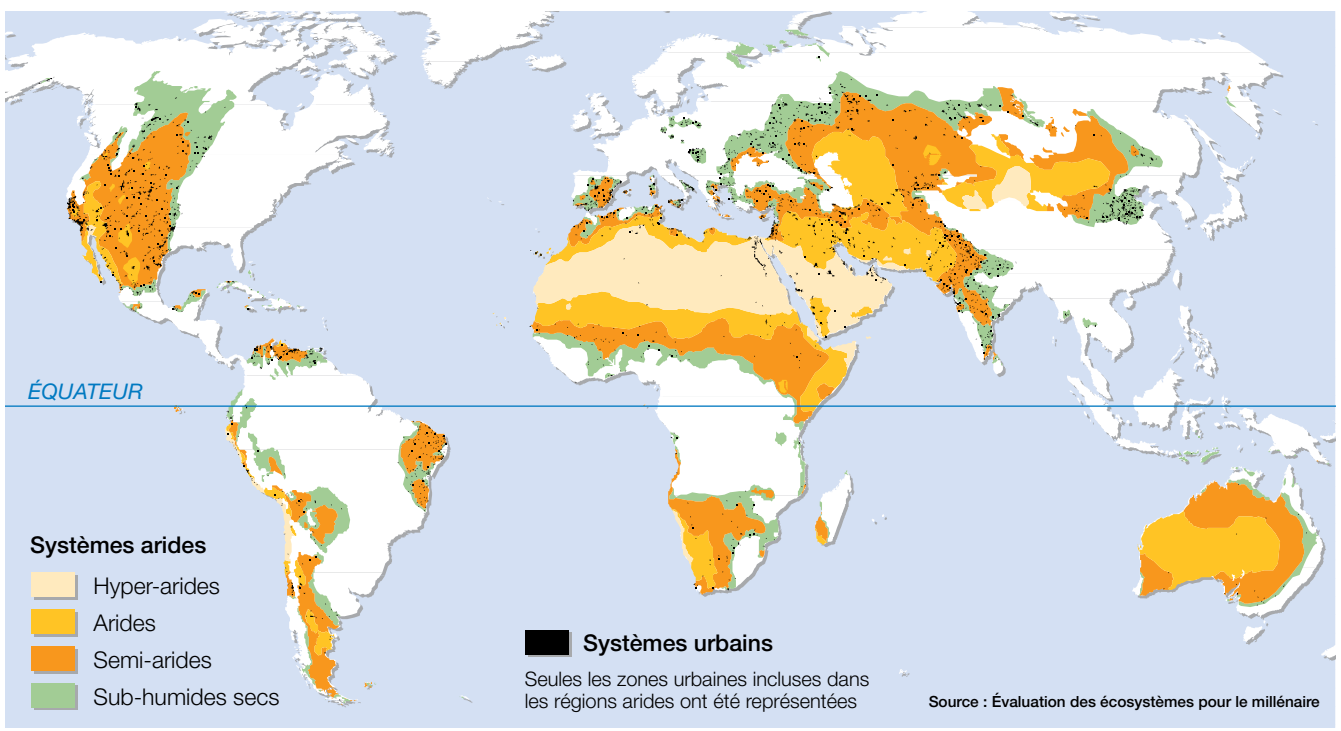

Figure 18.1 Distribution des zones arides du monde (MA, 2005a) 
particulièrement des prairies lorsque leur étendue et la composition des espèces sont déterminées par la rareté de l'eau.

La biodiversité des zones arides est bien adaptée à des conditions difficiles et, dans le monde entier, les zones arides sont des endroits importants pour l'endémisme. Les stratégies adaptatives des espèces vont des merveilles architecturales des termitières qui isolent les colonies contre des températures extrêmes aux amphibiens des déserts qui s'enfoncent dans le sable et restent dormants jusqu'à l'arrivée de la pluie. En fait, certaines de ces stratégies ont été à l'origine de découvertes importantes pour l'amélioration du mode de vie des hommes. Pourtant, avec les changements climatiques et la demande croissante des hommes pour ces systèmes arides, la biodiversité spéciale qui y vit est en grand danger.

Les zones arides abritent certaines des espèces les plus charismatiques, favorisent un fort taux d'endémisme des espèces et comprennent de nombreux écosystèmes et biomes uniques, comme les écosystèmes de type méditerranéen, les prairies, les savanes, les forêts sèches, les zones côtières, les déserts, le fynbos et le succulent Karoo (ces deux derniers étant des types de végétation très particuliers et uniques à l'Afrique $\mathrm{du}$ Sud) (Zeidler et Mulongoy ; 2003, White et al., 2000 ; Bonkoungou et Niamir-Fuller, 2001). De plus, de nombreux autres écosystèmes, comme des écosystèmes riverains ou forestiers, se trouvent dans des paysages arides et soumis aux risques de la dégradation des zones arides.

Les écosystèmes arides qui fonctionnent bien peuvent fournir de nombreux services écosystémiques comme des cultures vivrières, des plantes médicinales, du fourrage pour les animaux, des ressources génétiques, de l'eau pour les hommes ou les animaux, des matériaux de construction et des fibres textiles. Ils peuvent aussi être d'importantes sources de revenus (ex. par le tourisme) ou de soutien culturel et spirituel. La valeur potentielle de certains de ces services peut s'exprimer en termes de pourcentages de la production agricole dans les pays qui sont en grande partie arides. Par exemple, l'agriculture compte pour $30 \%$ du PIB en Afghanistan, au Kenya et au Soudan. Les parties arides de l'Inde contribuent à hauteur de $45 \%$ à la production agricole du pays. Les zones arides chinoises accueillent 78 millions de chèvres de race cachemire qui fournissent de 65 à 75\% du marché mondial, tandis que la Mongolie génère 30\% de son PIB grâce au pastoralisme en zone aride.

Le pastoralisme, qui est normalement plus profitable sur des terres peu propices aux cultures, est une source de moyens de subsistance importante en zones arides. Le pâturage itinérant permet une meilleure utilisation des terres pastorales qui sont soumises à des variations de pluviosité et de température. Les cultures et le pâturage sédentaire du bétail n'ont pas cette flexibilité de mouvements lorsque les conditions ne conviennent plus. Mais certaines pratiques traditionnelles permettent de faire des cultures grâce à la plantation d'arbres et à d'autres mécanismes qui favorisent la régénération naturelle.

Les espèces adaptées aux zones arides ont tendance à se montrer écologiquement résilientes et capables de s'adapter à des environnements extrêmes. Pourtant, selon l'Evaluation des écosystèmes pour le millénaire (EM), 10-20\% des zones arides sont dégradées, menaçant des milliards d'hectares de prairies et de cultures, et donc plus de 2 milliards de personnes qui vivent dans ces écosystèmes, des impacts qui en résulteront (chiffres de 2000).

\section{ZONES ARIDES ET DÉSERTIFICATION}

La désertification est un sujet de conversation de plus en plus fréquent en dehors de sa «terre natale » qu'est la Convention des Nations Unies 
sur la lutte contre la désertification (UNCCD). Si la désertification est un problème important pour les zones arides, la plupart d'entre elles ne sont pas désertes. L'UNCCD définit la désertification comme "la dégradation des terres [et de la végétation, l'érosion des sols et la perte de la couche arable et des terres fertiles] dans les zones arides, semi-arides et subhumides sèches». Cette dégradation s'exprime par une réduction persistante de la productivité biologique et écologique et elle peut se mesurer en suivant les extrants des services écosystémiques, dont les productions agricoles et la fourniture d'eau.

Les causes de la désertification incluent :

- Certaines politiques sociales et économiques

- L'obligation faite à des pasteurs nomades d'adopter une vie de cultivateur sédentaire

- Des pratiques foncières encouragées ou forcées qui aboutissent à la surexploitation des ressources

- Des pratiques non durables en matière d'aménagement du territoire, qui résultent souvent des trois points précédents.

La désertification touche tous les continents à l'exception de l'Antarctique et elle a des impacts particulièrement importants sur les pauvres des régions arides qui dépendent fortement des services écosystémiques que ces systèmes fournissent.

Beaucoup d'impacts de la désertification sur les zones arides s'exercent au niveau local, mais ils ont aussi des conséquences au niveau régional et mondial. Au point de vue de l'environnement, la perte de la végétation entraine celle du sol, l'érosion et des inondations. D'un point de vue social, les gens qui vivent dans des zones arides dégradées risquent d'être forcés de migrer vers d'autres régions qui sont déjà très peuplées et qui ne pourraient pas satisfaire une demande croissante.

D’autres effets de la dégradation des zones arides sur les modes de vie locaux sont :
- Perte des connaissances autochtones et du savoirfaire traditionnel ;

- Fragilité accrue des communautés se trouvant dans l'incapacité de s'adapter aux variations et aux changements de conditions ;

- Marginalisation des populations autochtones ;

- Conflits dans des zones arides et semi-arides ; et

- Disparition d'institutions de gestion traditionnelles qui ont fait la preuve de leur efficacité pendant des générations.

\section{PERTE DE LA BIODIVERSITÉ}

La perte de la biodiversité, critique dans ces environnements extrêmes, est ressentie particulièrement durement par les habitants des zones arides. La biodiversité des zones arides non seulement apporte son soutien aux populations locales mais est aussi une source de nombreux services pour les régions plus fortunées du monde. Pensons aux plantes médicinales comme Harpagophytum sp., ou Hoodia sp. utilisées pour soigner des maladies " occidentales » communes comme l'arthrite et l'obésité. Sans une gestion raisonnable des zones arides, les solutions aux problèmes de santé de demain risquent de disparaître avant même d'être découvertes (Encadré 18.1).

\section{PROBLÈMES URGENTS}

La désertification est accentuée par une suite de facteurs comme la rareté de l'eau, l'utilisation intensive des services écosystémiques et les changements climatiques. Ces facteurs sont étroitement liés puisque les changements climatiques vont probablement causer une raréfaction de l'eau dans de nombreuses régions arides, entraînant une diminution des services écosystémiques malgré une demande croissante. Ces changements pourraient aussi augmenter les risques de conflits. 
Encadré 18.1 Gomme arabique - une étude de cas de services écosystémiques de zones arides

\section{Gomme arabique dans des zones arides soudanaises}

Le type de forêt le plus important du Soudan est peut-être la ceinture de gommier arabique, située dans la zone de savane faiblement pluviale. Le hashab (Acacia senegal) fournit, dans cette ceinture, les services écosystémiques suivants :

- Il agit comme une barrière naturelle pour protéger plus de $40 \%$ de la surface totale du Soudan contre la progression du désert.

- Il soutient l'économie des familles en fournissant la gomme arabique ; c'est un arbre multifonctions qui a un rôle important pour les revenus des gens. Il procure aussi le bois de feu pour le ménage et du fourrage.

- Il enrichit la fertilité des sols, peut-être aussi par la fixation de l'azote.

Au temps des pharaons, la gomme arabique était utilisée pour l'embaumement des momies et pour fabriquer des aquarelles, des peintures et des teintures.

Traditionnellement, au Soudan, l'aménagement des terres comprenait un système de jachère qui permettait et les cultures et la récolte de gomme arabique. Le cycle de jachère commençait avec le défrichage d'un ancien jardin de gomme arabique (15-20 ans d'âge) pour y cultiver des denrées alimentaires. Les arbres étaient coupés à $10 \mathrm{~cm}$ du sol, et les troncs étaient laissés en place pour permettre la repousse de vigoureux rejets pendant que la surface dégagée était cultivée pendant 4-6 ans. Lorsque la fertilité du sol s'épuisait, on arrêtait les cultures et la surface était laissée en jachère à l'exception des arbres qui étaient incisés pour recueillir la gomme arabique jusqu'à l'âge de 15-20 ans. Le cycle pouvait alors se répéter. On considère que cette approche est une des formes les plus abouties de gestion de forêt naturelle en zone aride tropicale et on estime qu'elle est durable en termes de bénéfices environnementaux, sociaux et économiques.
Challenges actuels de la production de gomme arabique dans les zones arides soudanaises

L'importance de la gomme arabique pour la subsistance des populations qui habitent la ceinture de gommier est bien connue. Plus de quatre millions de personnes, dans cette ceinture soudanaise, sont actives dans l'incision, la récolte, le nettoyage et le commerce de la gomme. Le Soudan représente 70-80\% du marché mondial de la gomme arabique. Ses exportations annuelles vont de 20.000 à 50.000 tonnes, avec une moyenne, pour les dix dernières années, de 25.000 tonnes par an.

Ces dernières décennies, le système de jachère a été perturbé et la rotation traditionnelle cultures-jachères a été fortement écourtée, voire complètement abandonnée, avec de graves conséquences pour les récoltes vivrières et pour la production de gomme. La gestion durable des vergers de gomme est menacée par de graves sécheresses et par les coupes sans discernement des Acacia senegal pour le bois de feu et le charbon de bois. À ces menaces s'ajoute le fait que les communautés productrices ne disposent d'aucune infrastructure régulatrice, pas plus que d'informations sur le marché grâce auxquelles elles pourraient planifier la distribution et les ventes des produits à base de gomme. Citons encore le manque d'institutions financières et de transports. Par conséquent, un rapport notait que le revenu actuel des producteurs de gomme ne dépassait pas $40 \%$ du coût de production. Pour tenter de régler ces problèmes, des Associations des producteurs de gomme (APG) se sont créées à titre d'essai dans plusieurs provinces et elles se sont ensuite multipliées. Aujourd'hui, 1650 APG comptent deux millions de membres, dont $30 \%$ sont des femmes.

Source : A.G. Mohammed, 2008. 
Utilisation intensive des services écosystémiques, spécialement de l'eau

La croissance constante de la population et l'augmentation correspondante de la demande de nourriture pourraient bien augmenter la pression pour mettre davantage de terres à la disposition de l'agriculture et aboutir à une dégradation toujours plus grave et à des conflits entre groupes ethniques. Les régions arides connaissent des cycles de pénurie d'eau au cours desquels les populations locales sont plus vulnérables à leurs effets, à savoir les pénuries alimentaires et les crises sanitaires dues au manque d'eau.

\section{Changements climatiques}

Les effets des changements climatiques donnent une image complexe des possibilités des zones arides. Pour certaines, une sécheresse plus intense et prolongée risque d'éliminer toute productivité d'un paysage aride. Pour d'autres, une augmentation significative des précipitations (en intensité, en volume et en distribution spatiale des précipitations) pourrait transformer des zones arides en systèmes plus humides. Cela pourrait être bénéfique mais aussi entraîner des conflits entre agriculteurs et pasteurs.

La désertification contribue aux changements climatiques par la perte de sol et de végétation, qui diminue la capacité de stockage de carbone des sols. On estime que, chaque année, 300 millions de tonnes de carbone sont perdues dans l'atmosphère dans les zones arides suite à la désertification (près de $4 \%$ du total des émissions mondiales, toutes sources confondues) (EM, 2005a).

\section{GESTION DES ZONES ARIDES ET PRÉVENTION DE LA DÉSERTIFICATION}

\section{Des connaissances à creuser}

Un des plus importants efforts à fournir est d'améliorer la bonne compréhension des zones arides, des facteurs impliqués et de la vulnérabilité des populations locales qui en résulte. Les décideurs et les techniciens qui sont chargés de la conservation et qui imaginent des moyens de subsistance alternatifs doivent connaître avec précision le potentiel, les limites et les possibilités écologiques des terres arides et semi-arides afin de mieux comprendre les impacts urbains et extérieurs sur les terres arides et semiarides. Pour y répondre, l'Organisation des Nations Unies pour l'alimentation et l'agriculture (FAO), avec beaucoup d'autres partenaires, a développé l'Évaluation de la dégradation des terres dans les zones arides (LADA) qui comprend une cartographie, des indicateurs et des études pilotes au niveau des pays pour aider à améliorer nos connaissances des zones arides. L'UNCCD et l'Organisation météorologique mondiale (OMM) collaborent dans un projet d'aide en matière de prévention, d'alerte et de suivi de la sécheresse.

La valeur des zones arides et de leurs services écosystémiques doit aussi être mieux appréciée. Jusqu'ici, les zones arides se caractérisent toujours par un sous-investissement parce que leurs potentialités sont négligées en faveur des terres agricoles, des forêts tropicales ou des écosystèmes marins.

\section{GESTION INTÉGRÉE DES ZONES ARIDES}

\section{Gestion des ressources en eau}

La gestion intégrée des ressources en eau est un moyen clé d'empêcher la désertification en s'assurant que les politiques de gestion des sols sont adaptées aux traditions et aux besoins locaux. Ces politiques doivent favoriser le mode de vie actuel des pasteurs et préserver les connaissances traditionnelles; elles doivent éviter toute transition inutile vers des cultures plus gourmandes en eau. La gestion des zones arides implique une gestion de l'eau et exige que la coopération intersectorielle soit réelle. $\mathrm{La}$ réduction du stress sur les terres arides requiert parfois le développement et la promotion de 
moyens de subsistance alternatifs, notamment le déplacement vers des terres voisines, non arides.

\section{Restauration de zones arides dégradées}

Comme pour tout autre écosystème dégradé, la restauration de zones arides doit s'entreprendre à l'échelle du paysage, en utilisant les principes des approches par écosystème.

\section{Politique et gouvernance en zones arides}

La définition dogmatique d'une zone aride n'aide pas vraiment en termes de politique. En fait, la définition des terres arides de l'UNCCD differe de celle de la Convention sur la diversité biologique (CDB), la première étant plus précise quant au niveau des précipitations et la seconde englobant une plus grande surface par l'adjonction de types de végétation spécifiques (Encadré 14.1). Cela peut causer une certaine confusion chez les parties qui essaient de réaliser des programmes de travail dans les zones arides pour les deux conventions, et c'est un problème typique qui souligne bien le besoin d'harmonisation entre les accords multilatéraux sur l'environnement (AME).

Cependant, quelle que soit la définition, les problèmes de gouvernance rencontrés par la gestion des zones arides restent les mêmes :

- Corriger le manque de représentativité des populations des zones arides, sécuriser leurs droits fonciers et les problèmes relatifs d'autodétermination, d'éducation et de santé

- Décentraliser la gestion des ressources naturelles, notamment établir des « conventions locales » (des accords communautaires) et permettre aux gens de recevoir des compensations pour les services écosystémiques fournis par les zones arides et

- Renforcer la résilience des habitants des zones arides, notamment les pasteurs nomades, grâce à des Cadres politiques et des mesures pertinents.

Comme le soulignent certains chapitres précédents, ces problèmes de gouvernance devront aussi aborder les sujets concernant les changements climatiques (Chapitre 5) et la réduction de la pauvreté (Chapitre 1).

Les zones arides sont des écosystèmes productifs qui font vivre un grand nombre de personnes, mais ces personnes sont sensibles aux climats, aux marchés et aux droits changeants (Mortimore et al., 2008). La gestion efficace des zones arides - et donc la prévention de la désertification - sera une étape importante vers la réduction de la pauvreté et la conservation de la biodiversité dans une partie significative de notre monde. 


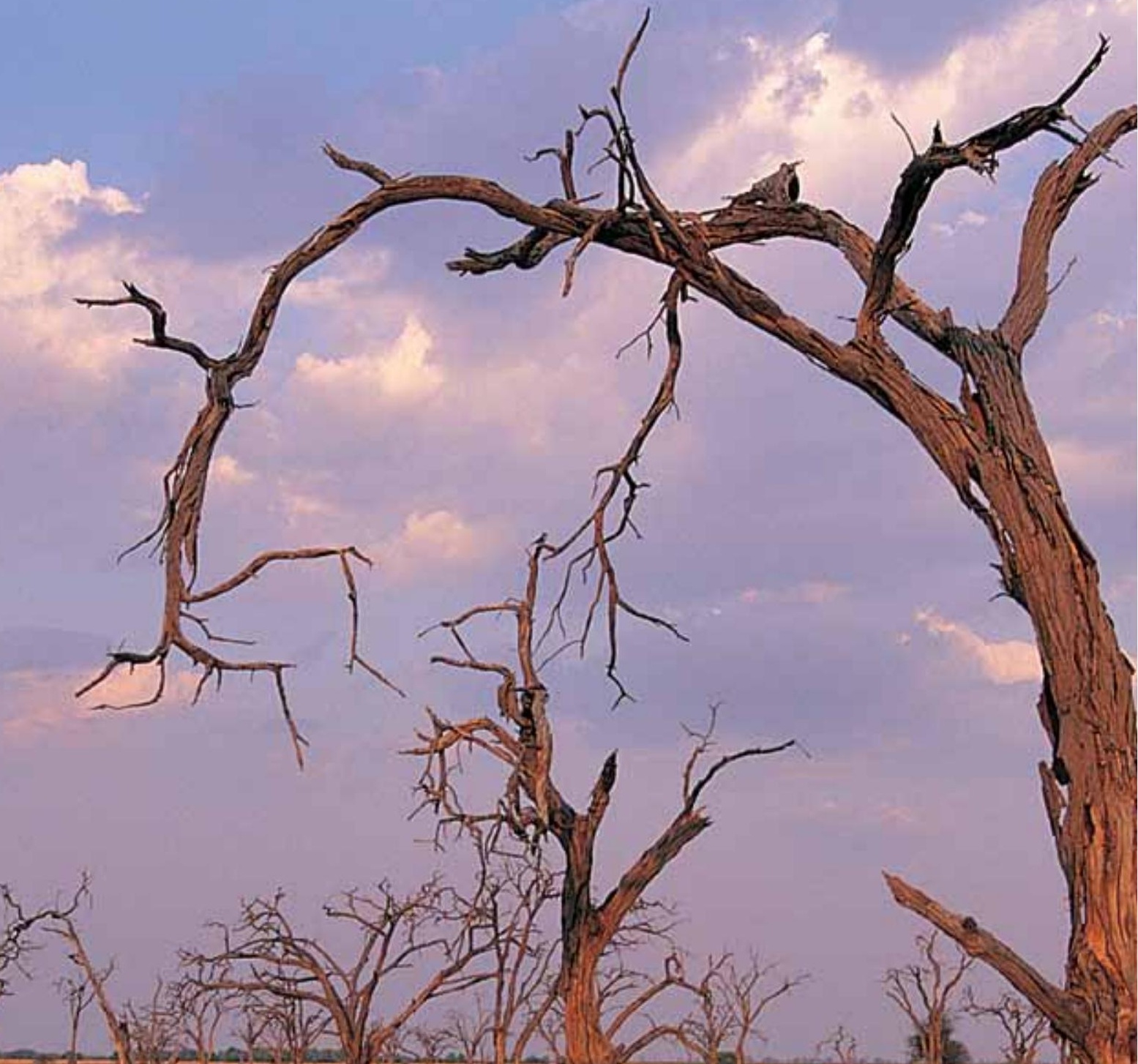

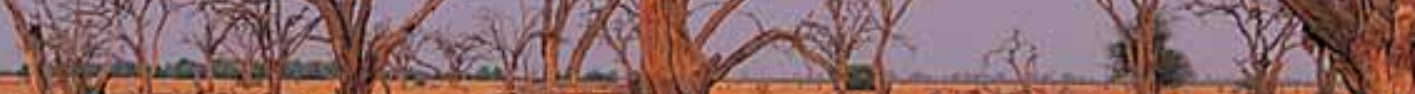

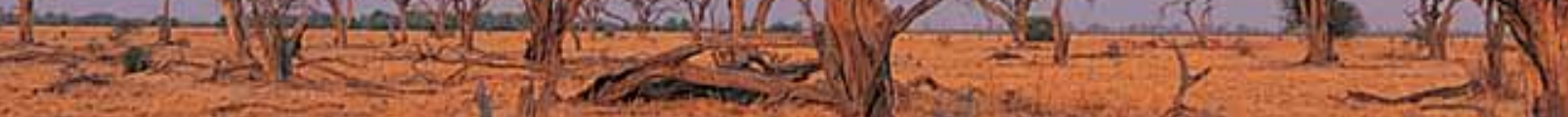

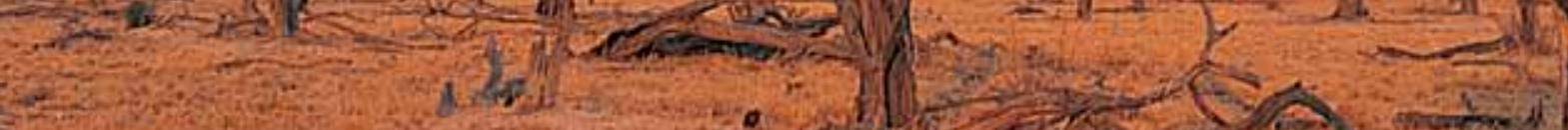

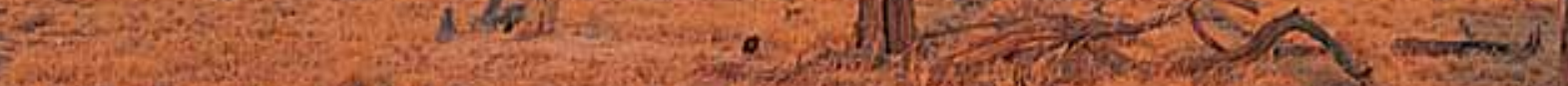

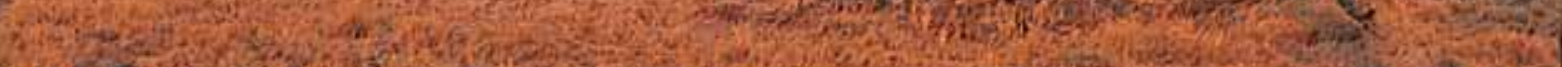

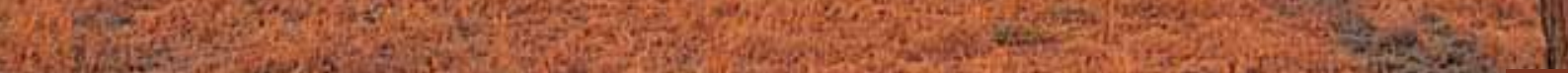
2. 1.

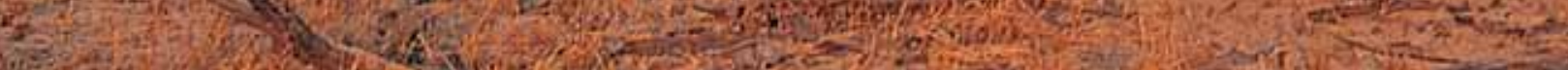

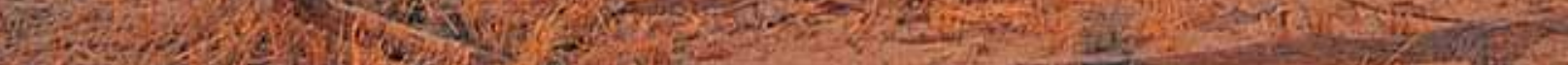

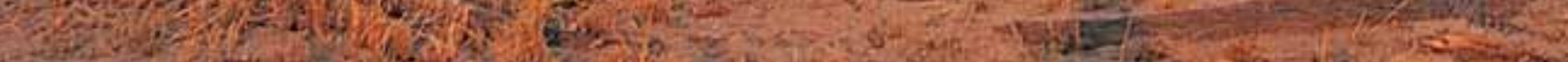
3. 


\section{Systèmes d'eau douce : gérer les flux 19. pour les hommes et pour la nature}

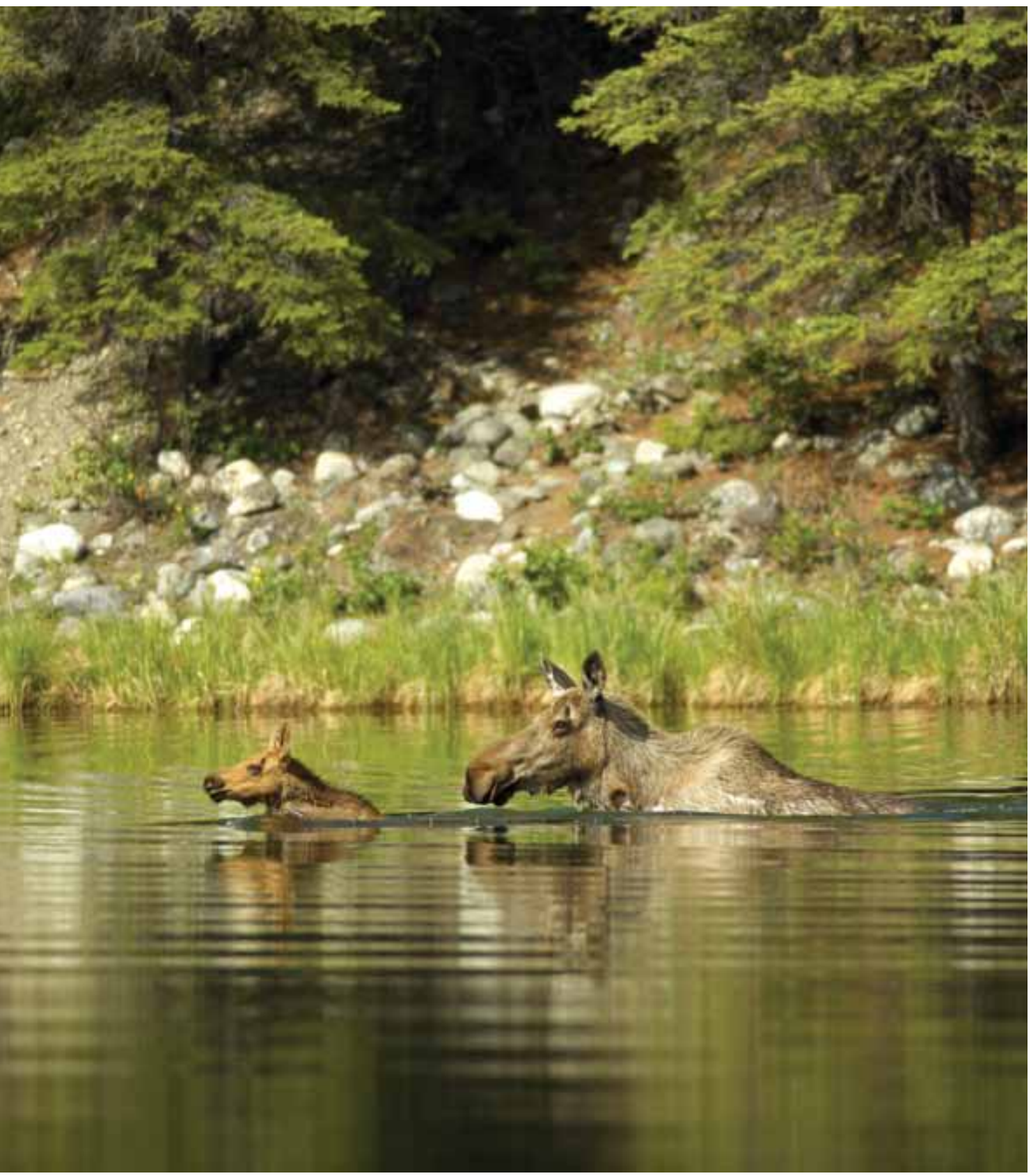


Les systèmes d'eau douce couvrent moins d'un pour cent de la surface terrestre et pourtant ils sont essentiels pour maintenir la vie. La qualité de l'eau détermine la santé des hommes et des écosystèmes. Les cours d'eau et les nappes phréatiques requièrent une approche holistique, à l'échelle du paysage, pour traiter les pressions qui s'exercent sur les ressources tant en amont qu'en aval ; une approche qui reconnaisse l'importance des valeurs esthétiques, religieuses, historiques et archéologiques de la contribution de l'eau au patrimoine d'un pays.

Les habitats d'eau douce hébergent 126.000 espèces, soit $7 \%$ des quelque 1,8 million d'espèces décrites, dont un quart des 60.000 vertébrés environ (Balian et al., 2008). Ils ont aussi une valeur économique. Selon une estimation, les biens et services fournis par les zones humides du monde entier équivaudraient à 70 milliards de dollars par an (Schuyt et Brander, 2004).

La biodiversité et le bien-être sont touchés par les changements qui affectent l'eau douce. En moyenne, les populations d'espèces d'eau douce ont été réduites de moitié entre 1970 et 2005 , un déclin plus rapide que dans d'autres biomes (Programme mondial pour l'évaluation des ressources en eau - WWAP, 2009). L'Indice de la Liste rouge pour les oiseaux qui vivent dans des habitats d'eau douce montre un des déclins les plus graves, tous habitats confondus, juste derrière celui des habitats marins (Butchart $e t$ al., 2004). Une évaluation mondiale pour la Liste rouge des crabes d'eau douce a révélé que, chez les espèces pour lesquelles les données disponibles étaient suffisantes pour faire une évaluation, 32\% étaient menacées (Cumberlidge et al., 2009). L'examen du statut des poissons d'eau douce dans des régions particulières donne des chiffres qui vont de 11\% d'espèces menacées en Afrique australe (Darwall et al., 2008) à 56\% des poissons d'eau douce endémiques de la région méditerranéenne qui sont menacés (Smith et Darwall et al., 2006).

Plus de 60\% des 227 plus grands cours d'eau sont entrecoupés de barrages, de dérivations ou de canaux (Revenga et al., 2000) qui entraînent une dégradation générale des écosystèmes d'eau douce. La surpêche et des pratiques de pêche destructrices, la pollution, les espèces envahissantes et les changements climatiques sont d'autres motifs graves d'inquiétude pour la plupart des systèmes d'eau douce. Darwall et al. (2008) rapportent que $85 \%$ des poissons menacés d'Afrique australe, $55 \%$ des poissons d'eau douce menacés en Europe et un peu moins de 45\% des poissons d'eau douce menacés à Madagascar sont affectés par des espèces envahissantes. Dans le dernier cas, il s'agit en grande partie du résultat d'un programme de restauration des pêcheries locales qui a eu recours à la réintroduction de 24 espèces de poissons non indigènes (Benstead et al., 
2003). Les changements climatiques vont accroître la vulnérabilité et auront d'autres impacts sur les systèmes d'eau douce. Enfin, dans de nombreux pays, la politique et les lois en matière d'eau sont en train de connaitre des réformes et elles devront être appliquées rigoureusement pour préserver les ressources en eau.

Dans un monde où l'accès à l'eau se réduit, pour résoudre les problèmes de conservation, il faut des solutions qui combinent les besoins des hommes et de la nature. La Vision de l'eau et de la nature (2000) encourage une approche par écosystème pour la gestion intégrée des ressources en eau (GIRE), notamment par l'amélioration de la gouvernance de l'eau, le renforcement du rôle des parties prenantes, l'élaboration d'un pôle de connaissance et la valorisation des ressources en eau.

L'UICN a préparé une série d'outils pour favoriser la gestion raisonnable des ressources en eau afin de renforcer la sécurité hydrique : les titres en sont Changez, Débit, Value, Pay, Share et Rule. Ils sont accessibles en ligne et disponibles en plusieurs langues sur :http://www.iucn.org/ about/work/programmes/water/wp_resources/ wp_resources_toolkits/.

\section{SERVICES ÉCOSYSTÉMIQUES ET SÉCURITÉ HYDRIQUE}

Les hommes ont besoin d'un minimum de 20 litres d'eau par jour pour boire, se baigner et maintenir une hygiène de base (UN-Water, 2007). Imaginez ce que cela signifie de survivre avec un quart seulement de cette quantité, cinq litres par jour - la quantité dont disposaient les gens durant la sécheresse en Afrique de l'Est (2005-2006). Les Nations Unies déclarent qu'en 2025, les deux tiers d'entre nous connaitront des pénuries d'eau et qu'un manque d'eau sévère affectera la vie et la subsistance de 1,8 milliard de personnes (UN-Water, 2007).

Les défis à relever concernent la quantité et la qualité. L'évaluation mondiale des eaux internationales 2006 a confirmé que les pénuries d'eau étaient un problème dans la plupart des régions du monde, mais spécialement en Afrique subsaharienne où ces pénuries touchaient neuf des 19 systèmes d'eau douce évalués cette annéelà et que la pollution, notamment la pollution transfrontalière, affectait cinq systèmes. D'ici 2025, on s'attend à ce que de nombreuses régions du Sud connaissent des pénuries d'eau (voir Figure 19.1). Cependant, la rareté de l'eau n'est continue ni dans le temps, ni dans l'espace. Le manque physique d'eau survient lorsque l'accès physique est réduit, c.-à-d. quand le développement des ressources en eau approche ou a dépassé les limites soutenables. Le manque économique d'eau existe lorsque la population n'a pas le capital humain, institutionnel et économique nécessaire pour accéder à l'eau même quand il y a dans la nature assez d'eau disponible pour répondre à la demande humaine. La pénurie économique due à une distribution inégale des ressources a de nombreuses causes, comme des conflits politiques et ethniques. Une grande partie de l'Afrique subsaharienne souffre des effets de ce type de pénurie d'eau (Evaluation globale de la gestion de l'eau en agriculture, 2007).

La crise de l'eau provient d'une demande croissante, de la détérioration de la qualité et donc d'un effondrement de la quantité disponible par personne. La différence de sécurité hydrique entre le Japon et le Cambodge - qui ont la même pluviosité moyenne de $160 \mathrm{~cm}$ par an - est due au fait que le Japon a su créer des infrastructures pour retenir et stocker l'eau. Dans les pays où les chutes de pluie sont abondantes, comme le Bangladesh et la Birmanie, une grande partie des pluies de la mousson ne sont pas récoltées pour être utilisées et elles s'écoulent jusqu’à l'océan.

Alors que le minimum d'eau nécessaire est de 20 litres par jour, la consommation moyenne aux Etats-Unis et dans les pays européens est 
Peu ou pas de pénurie d'eau

Pénurie physique d'eau

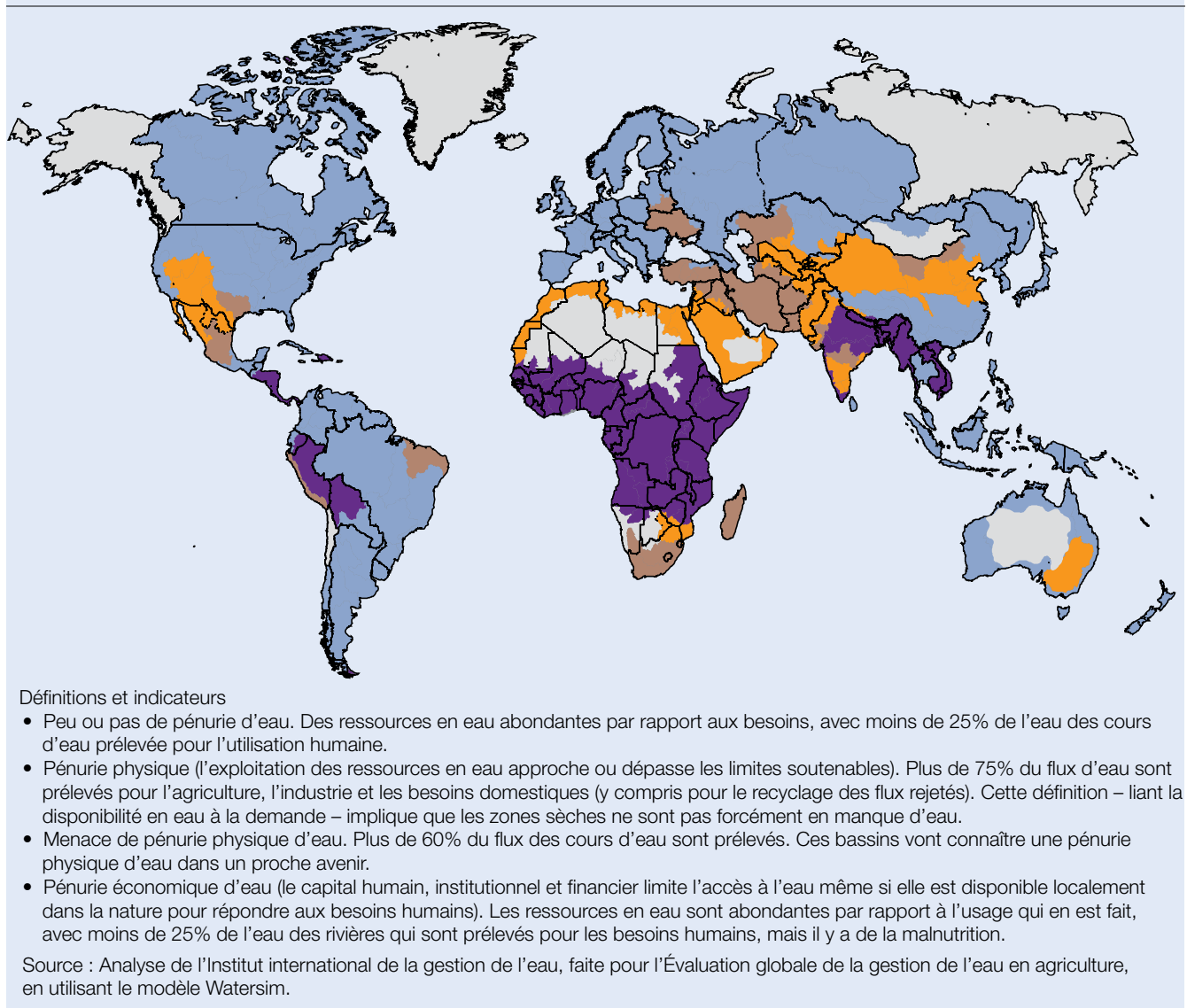

Figure 19.1 Pénuries d'eau prévues en 2025 (IWMI, 2009)

comprise entre 200 et 600 litres (UN-Water, 2007). Gérer votre consommation d'eau personnelle peut être aussi facile que fermer le robinet en vous lavant les dents. Un outil peut servir à déterminer la consommation d'eau, c'est l'empreinte hydrique (Encadré 19.1). L'empreinte hydrique d'un individu, d'une communauté ou d'une entreprise est définie par le volume total d'eau douce utilisé pour produire les biens et services consommés par chaque personne ou par chaque communauté ou produits par l'entreprise. L'outil Water footprint et d'autres approches peuvent être utilisés pour appliquer la GIRE.

\section{Encadré 19.1 Marcher sur l'eau :} comment est votre empreinte hydrique?

Beaucoup d'entre nous ont entendu parler du bilan carbone, mais peu savent qu'ils ont aussi une empreinte hydrique. Vous pouvez calculer combien d'eau exigent vos habitudes de consommation - que ce soit pour la douche, la cuisson, la nourriture, ou le type d'électricité utilisée. Visitez le site du réseau Water footprint pour en savoir plus : http:// www.waterfootprint.org/?page=files/home. 


\section{GESTION DE L'EAU ET FLUX ENVIRONNEMENTAUX}

La GIRE est « un processus qui favorise le développement et la gestion coordonnés de l'eau, des territoires et des ressources qui s'y rapportent. Elle vise la maximisation du bienêtre économique et social de manière équitable tout en préservant les écosystèmes vitaux " (GWP, 2009). Elle intègre la gestion à l'échelle du paysage, une échelle suffisamment large pour pouvoir reconnaître le rôle de toutes les parties prenantes et les facteurs influents critiques qui façonnent les décisions en matière d'aménagement du territoire. La GIRE est fondée sur les Principes de Dublin (GWP, 2000) à savoir :

Principe 1 : L'eau douce est une ressource limitée et vulnérable

Principe 2 : Une approche participative

Principe 3 : Le rôle important des femmes

Principe 4 : L'eau est un bien public qui a une valeur économique

Les Membres de l'UICN, dans la Résolution 4.063 (La nouvelle culture de l'eau - La gestion intégrée des ressources en eau) ont pressé les gouvernements d'adopter la GIRE et de favoriser les Cadres nécessaires à son application.

La question clé, lorsque l'on gère les allocations d'eau, est de savoir " comment nous pouvons garantir qu'il y a assez d'eau pour la nature ». Il est possible d'y répondre en appliquant les flux environnementaux. Les flux environnementaux décrivent la quantité, la périodicité et la qualité des débits d'eau nécessaires pour préserver les écosystèmes d'eau douce et les estuaires, ainsi que les moyens de subsistance et le bien-être des personnes qui dépendent de ces écosystèmes (Déclaration de Brisbane, 2007). L'on entreprend des évaluations pour déterminer le flux nécessaire pour garder un cours d'eau en bon état et favoriser les services écosystémiques vitaux. Cette information sert à prendre des décisions informées pour les allocations d'eau à tous les secteurs, y compris l'environnement.

Pour améliorer l'intégration des flux environnementaux dans les politiques et dans les pratiques de gestion de l'eau, il faut communiquer, apprendre et prouver les bénéfices des flux pour les populations et pour la nature. Le Réseau des flux environnementaux (www. eflownet.org) est un point de référence central pour toute information sur les flux et c'est aussi un outil permettant de partager des expériences, de développer des concepts et d'être relié à un large public intersectoriel.

L'UICN encourage l'application des flux environnementaux pour atténuer les effets du développement d'infrastructures sur les cours d'eau, comme les barrages et les vastes zones d'irrigation. Les flux environnementaux sont réalisés en changeant le fonctionnement des infrastructures de façon à restaurer la quantité, la qualité et le rythme saisonnier du débit des cours d'eau pour qu'en aval, les écosystèmes et les services qu'ils fournissent aux gens soient préservés. L'application des flux environnementaux passe par des négociations entre parties prenantes sur les allocations de l'eau, qui encouragent l'intégration des besoins des gens et de la nature dans les décisions concernant la gestion des ressources en eau. En consolidant l'appui à l'application des flux environnementaux dans les politiques et les lois, on suscite le développement des connaissances, des capacités et des institutions nécessaires pour mettre en œuvre la GIRE.

\section{GOUVERNANCE DE L'EAU ET PARTICIPATION DES PARTIES PRENANTES}

Une gestion efficace de l'eau doit être étayée par des politiques et des lois qui permettent une définition transparente des droits, des rôles et des responsabilités de chacun, notamment en vue d'une attribution d'eau suffisante pour maintenir les écosystèmes en bon état. Pour réussir, la mise 
en œuvre de politiques et de lois bien structurées en matière d'eau requiert aussi des institutions régulatrices, ainsi qu'un environnement favorable qui se caractérise par sa transparence, sa fiabilité, sa responsabilité et par l'absence de corruption.

Ceci fut reconnu au niveau international lors de l'Assemblée du millénaire des NU 2000, qui a accepté de « mettre fin à l'exploitation non durable des ressources en eau, en développant des stratégies de gestion de l'eau aux niveaux régional, national et local, qui encouragent un accès équitable et une fourniture suffisante ». Au Sommet mondial du développement durable (SMDD) de 2002, les Chefs d'État se sont mis d'accord sur une cible spécifique pour préparer la Gestion intégrée des ressources en eau (GIRE) et des plans d'efficience pour l'eau pour 2005 - une cible qui ne fut pas atteinte.

La gouvernance de l'eau reste un défi majeur pour de nombreux pays, notamment à cause du manque de cohérence entre les secteurs, et de politiques et de lois contradictoires décrétées à différentes époques par des administrations et des groupes d'intérêt différents. Réformer les politiques et les lois nationales en un ensemble cohérent est une tâche difficile et coûteuse, mais les pays qui l'ont fait trouvent que leurs plans de mise en œuvre s'effectuent ensuite plus aisément. Le Brésil, par exemple, a entrepris une longue réforme de sa structure de gouvernance de l'eau qui, suite à la réorganisation systématique de sa politique, de ses lois et de ses institutions, a entraîné une amélioration substantielle de son programme de gestion de l'eau. De plus, Iza et Stein (2009) suggèrent que les réformes de la gouvernance de l'eau qui réduisent la pauvreté et font réaliser des économies devraient se fonder sur des principes d'équité et de durabilité. Par exemple, l'Afrique du Sud a accompli d'ambitieuses réformes dans le domaine de l'eau ces dernières années. Le National Water Act garantit une « réserve d'eau » pour couvrir la fourniture d'eau de base et assurer le bon état des écosystèmes aquatiques.

Les Membres de l'UICN, dans la Résolution CMN 3.006 (Protection des eaux de la planète dans l'intérêt écologique et public) ont accordé un soutien d'urgence pour atteindre la cible du SMDD et pour arriver à une participation complète dans les prises de décisions sur la conservation, la protection, la distribution et l'utilisation de l'eau. La communauté internationale encourage aussi les approches de la gestion de l'eau fondées sur les droits, dont le droit fondamental à de l'eau propre et potable. Au niveau national, l'Etat doit traduire dans la pratique ces obligations et ces engagements pris dans un contexte international.

La transformation de la politique et de la gestion de l'eau résulte d'un consensus élaboré dans des plateformes réunissant de nombreuses parties prenantes. Ces plateformes donnent aux parties prenantes issues du niveau local, du bassin hydrologique ou transfrontalier, le pouvoir de se mettre d'accord sur des droits, des rôles et des responsabilités et de négocier des réformes de la législation sur l'eau.

De plus, un système de bonne gouvernance doit " penser à l'échelle du bassin hydrologique, mais agir à l'échelle locale ». Lorsque des associations de base des utilisateurs de l'eau sont impliquées dans le processus d'évaluation, l'exécution et la maintenance des systèmes de captage d'eau traditionnels, ceux-ci sont plus résilients et permettent aux communautés de s'adapter aux changements climatiques. Impliquer la société civile à tous les niveaux favorise la prise de conscience et de responsabilité vis-à-vis de l'eau et facilite l'acceptation du système juridique. Cela offre alors une plateforme très utile pour résoudre d'éventuels conflits entre droits traditionnels et coutumiers, en facilitant la mise en application de la loi sur l'eau grâce à la participation active des utilisateurs qui sont au bout de la chaîne de la distribution d'eau. 
Enfin, ils peuvent jouer un rôle très important en surveillant leur part du système hydrique.

La bonne gouvernance et la bonne gestion de l'eau dépendent de l'implication des femmes. Une étude des projets de fourniture d'eau et d'assainissement dans 88 communautés de 15 pays, réalisée en 1988 par le Centre international de l'eau et l'assainissement (IRC), a découvert que les projets conçus et réalisés avec la pleine participation des femmes étaient plus durables et efficaces que ceux qui n'intégraient pas les femmes comme des partenaires à part entière (IRC, 1988).

La gouvernance des eaux transfrontalières est un problème complexe où la réalisation des objectifs environnementaux représente plusieurs défis. Il existe plus de 260 cours d'eau internationaux, dont les bassins couvrent $45 \%$ de la surface terrestre et qui totalisent près de $80 \%$ du débit mondial des cours d'eau. Près de $90 \%$ de la population mondiale vivent actuellement dans les pays qui partagent ces cours d'eau (Banque mondiale, 2009). Ces ressources essentielles sont soumises à une pression croissante parce que les populations s'accroissent et que les économies se développent. Il est important d'identifier les mécanismes et les instruments qui privilégient le fait de voir l'utilisation de l'eau comme un catalyseur de coopération régionale plutôt que comme une source potentielle de conflits. La gestion et le développement participatifs de ces cours d'eau exigent de grandes compétences, des institutions solides, des investissements conséquents et une forte coopération transfrontalière. Les institutions qui font exactement cela sont, par exemple, le Dialogue sur le Bassin du Nil, la Commission du Mékong, et l'Autorité du Bassin de la Volta récemment constituée.

Trouver une approche commune de la gouvernance des eaux transfrontalières est plus compliqué du fait que les législations, les pratiques de gestion de l'eau, les structures institutionnelles, les langues et les cultures sont différentes d'un côté de la frontière à l'autre. Néanmoins, la coopération pour gérer la qualité et la quantité des masses d'eau transfrontalières offre aussi une opportunité dont peuvent profiter toutes les parties impliquées (Aguilar et Iza, 2006). Les négociations, les consensus et les accords atteints entre deux parties ou plus d'un bassin de rivière partagé deviennent des parties du système de gouvernance de l'eau, mais c'est la volonté politique des états souverains qui détermine si ceux-ci vont réussir à soutenir une gestion durable de l'eau.

\section{PAIEMENTS POUR LES SERVICES DES BASSINS HYDROGRAPHIQUES}

Les ressources en eau sont à la base de l'économie, et les dividendes des investissements dans les services hydrologiques doivent tenir compte des bénéfices et de la sécurité hydrique pour la subsistance, les affaires et le développement économiques. Dans le secteur des affaires, l'eau a des avantages divers : les intérêts des services de l'eau (les personnes qui tirent des revenus de l'eau); les sociétés qui vendent des produits qui nécessitent de l'eau ; les compagnies hydroélectriques; les sociétés qui fabriquent des biocarburants ; des sociétés productrices d'énergie qui ont besoin d'eau pour leur refroidissement ; les industries qui ont besoin d'eau pour fonctionner, etc. Avant de se lancer dans les affaires, il faut cependant que les utilisateurs comprennent parfaitement toutes les pertes potentielles de services écosystémiques que ces développements peuvent entraîner. Les incitants financiers, dont les paiements pour les services écosystémiques (PES), font partie du financement durable de la GIRE. En Equateur, le Fonds pour l'eau de Quito (FONAG) a lancé un prospectus d'investissement pour attirer les contributions des secteurs public et privé dans un fonds à long terme qui vise à sécuriser la quantité et la qualité de l'eau fournie à Quito par le Bassin de la Guayllabamna. 
L'eau est une ressource vitale pour les secteurs de l'agriculture et de l'énergie dans le monde entier. L'agriculture est, de loin, le plus gros utilisateur d'eau. L'irrigation et le bétail représentent $70 \%$ des prélèvements d'eau, $80 \%$ même dans certaines régions, si bien que c'est avec le secteur agricole que les conservationnistes devraient se rapprocher davantage pour approfondir leurs connaissances des problèmes d'eau (EM, 2005a ; Programme mondial pour l'évaluation des ressources en eau - WWAP, 2009). Sans accès fiable à de l'eau de qualité et en quantité suffisantes, la production d'électricité ne marche pas, surtout lorsque le débit ou le refroidissement des stations hydroélectriques sont réduits. Ces secteurs, y compris le nombre croissant de producteurs de biocarburants, doivent faire une priorité de la fourniture d'eau durable à l'avenir, notamment grâce à des investissements dans la gestion durable des bassins hydrographiques. Les politiques de l'eau et de l'énergie doivent être coordonnées, aussi bien pour leurs stratégies que pour leur fonctionnement.

Les retours sur investissements dans la gestion de l'eau et dans les services écosystémiques sont trop souvent ignorés ou sous-estimés. La gestion fondée sur les services écosystémiques peut constituer un Cadre dans lequel inscrire les prises de décisions concernant les services fournis par les systèmes naturels et pour identifier les compensations qui pourraient être nécessaires dans ces décisions (Farber et al., 2006). Les investissements dans la durabilité d'un bassin de rivière stimulent la « croissance verte » et la résilience économique. L'eau et les services fournis par les bassins hydrographiques, comme le stockage de l'eau, sa purification, la régulation de son débit et la sécurité alimentaire, sont des bénéfices pour toute l'économie, du niveau local au niveau national. Les investissements qui assurent la poursuite ou le retour de la sécurité hydrique et des services des bassins hydrographiques soutiennent les moyens de subsistance locaux, créent des opportunités pour le développement d'entreprises et sont à la base de la croissance économique nationale. Les investissements dans la durabilité de bassins hydrographiques peuvent donc stimuler une croissance favorable aux plus pauvres et écologiquement solide, tout en renforçant la résilience des communautés et des économies nationales.

\section{EAU ET CHANGEMENTS CLIMATIQUES}

L'on prévoit que les changements climatiques vont avoir des impacts importants sur les ressources en eau et sur des fragilisations généralisées. Ces effets se feront ressentir d'abord et surtout au niveau de l'eau - des sécheresses, des inondations, des tempêtes, la fonte des glaces et la hausse du niveau des mers. La réduction rapide des glaciers de l'Himalaya, qui pourraient avoir perdu quatre cinquièmes de leur surface d'ici 2030, signifie qu'un réservoir naturel qui stocke l'eau pour plus d'un milliard de personnes pourrait disparaître.

Traiter ces impacts signifie que nous avons besoin de stratégies pour nous adapter aux changements climatiques. Si l'eau est au centre des impacts des changements climatiques, elle est aussi au centre des politiques, de la planification et des actions d'adaptation. Les bassins hydrographiques, les côtes et leurs écosystèmes sont des infrastructures naturelles qui peuvent atténuer ces impacts.

Ils permettent de stocker l'eau, de contrôler les débits et de protéger les côtes, toutes interventions vitales pour réduire la vulnérabilité des communautés et des économies face aux changements climatiques. Un investissement dans la GIRE, en tant que « infrastructure naturelle critique pour la nation ", devrait faire partie intégrante de tout portefeuille d'adaptation aux changements climatiques (Smith et Barchiesi, 2008). 


\section{Systèmes agricoles : la biodiversité 20. dans des paysages domestiqués}


Alors que la population mondiale se dirige, inexorablement semble-t-il, vers les neuf milliards d'hommes, l'on accepte généralement que la production mondiale de nourriture va devoir augmenter d'au moins 50\% pour nourrir tout le monde et améliorer les normes de vie de milliards de personnes. Plus difficile encore, ceci doit se faire dans le contexte des changements climatiques, qui rendent la productivité agricole hautement aléatoire. La demande de nourriture pourrait croitre plus vite encore que la population humaine, à cause d'une urbanisation en hausse, de revenus plus importants, et des plus grands efforts réalisés pour réduire la faim chez les 950 millions de personnes que l'on estime actuellement dénutries (FAO, 2008c). La consommation mondiale de bétail devrait dépasser 650 millions de tonnes d'ici 2020. II faudra certainement plus de terres pour faire pousser les cultures et pour nourrir le bétail, et davantage encore si les biocarburants doivent devenir un plus grand contributeur à la production de l'énergie nécessaire. Rien qu'en Afrique, la surface vouée à la production de céréales devrait passer d'un peu plus de 100 millions d'hectares en 1997 à environ135,3 millions d'hectares en 2025, ce qui va inévitablement devoir donner lieu à des compromis entre terres affectées aux cultures, à l'élevage et à d'autres usages.

Nourrir une population humaine de neuf milliards de personnes en employant les méthodes actuelles pourrait exiger de convertir un autre milliard d'hectares d'habitats naturels en terres agricoles, surtout dans les pays en développement, tout en doublant ou triplant les apports d'azote et de phosphates, en doublant la consommation d'eau et en triplant l'usage de pesticides. Le facteur limitant le plus sérieux est l'eau, étant donné que 70\% de l'eau douce utilisée par les hommes passent déjà dans l'agriculture. Les scénarios proposés par l'Evaluation des écosystèmes pour le millénaire (EM) supposent que la production agricole du futur devra se focaliser plus précisément sur des systèmes de gestion sensibles aux questions d'environnement, et qui accordent plus d'attention à la biodiversité (Carpenter et al., 2005).

Qu'une plus importante production agricole soit atteinte par une utilisation plus intensive des terres agricoles existantes ou par une utilisation 
plus extensive de terres qui servent aujourd'hui à d'autres usages, la biodiversité sera inévitablement soumise à des pressions croissantes.

On peut définir l'agriculture comme l'art, la science et l'entreprise de production du bétail et de culture des sols pour produire des récoltes. Elle dépend totalement de gènes, d'espèces, d'écosystèmes et de leur variabilité. Cette biodiversité aussi fournit à l'agriculture la capacité de s'adapter à des conditions changeantes.

Le mouvement de la conservation étudie maintenant comment il veut se positionner vis-à-vis de l'agriculture de la façon la plus productive possible. Après tout, les agriculteurs, les pasteurs et les chasseurs-cueilleurs sont les occupants des paysages ruraux, où survit la plus grande partie de la biodiversité mondiale. Si nous espérons préserver la biodiversité mondiale, et un équilibre raisonnable entre les hommes et le reste de la nature, l'agriculture doit faire partie de la discussion.

D'autre part, la conservation a de nombreux atouts pour contribuer à une agriculture durable. Cette agriculture devra être très diversifiée, ce qui implique des écosystèmes favorables qui renferment profusion d'espèces sauvages pour le plus grand profit de l'agriculture. Celles-ci comprennent les parents sauvages des plantes cultivées, des pollinisateurs, des espèces utiles pour le contrôle des nuisibles, les microorganismes du sol et beaucoup d'autres.

Près d'un tiers de la surface de notre planète est dominé par des productions agricoles ou des pâturages plantés qui ont donc un effet écologique profond sur le paysage dans son ensemble. Dix à 20\% de terres supplémentaires sont consacrées au pâturage extensif du bétail, et quelque 1-5\% de nourriture est aussi produite dans des forêts naturelles (Cassman et Wood, 2005). La biodiversité et les services écosystémiques impliquant l'agriculture sont donc critiques pour assurer un avenir durable à nos agriculteurs.

\section{COMMENT LA BIODIVERSITÉ FAVORISE LA DEMANDE CROISSANTE POUR LES PRODUCTIONS AGRICOLES}

Pratiquement toutes les espèces domestiquées d'animaux et de plantes ont encore, dans la nature, des parents dont la diversité génétique est très intéressante parce qu'elle pourrait permettre aux espèces domestiquées de s'adapter à des conditions changeantes. Si les banques nationales et internationales de semences conservent un matériel génétique de grande valeur, les " cousins " sauvages sont particulièrement importants parce qu'ils vivent et s'adaptent aux nouvelles conditions induites par les changements climatiques, alors qu'ils sont en compétition avec d'autres espèces, avec des prédateurs et de nouvelles maladies. Les efforts pour préserver les parents sauvages des plantes et des animaux domestiqués ont beaucoup augmenté ces dernières décennies; des accords internationaux reconnaissent leur importance; de nombreux projets ont été lancés dans divers pays et la collaboration internationale s'intensifie (Meilleur et Hodgkin, 2004). À l'UICN, la Commission de sauvegarde des espèces (CSE) a désormais un Groupe de spécialistes qui travaille sur les plantes sauvages apparentées à des plantes cultivées, et plusieurs de ses autres groupes de spécialistes s'occupent des parents sauvages d'animaux domestiqués (ex. bovins sauvages, camélidés, suidés, faisans).

Un service d'appui particulièrement important fourni à l'agriculture par la biodiversité est la protection des plantes. Les plantes répondent aux insectes qui se nourrissent de leurs feuilles en synthétisant et en diffusant des mélanges complexes de composés volatils ; ceux-ci attirent des insectes qui sont les ennemis naturels des premiers et qui aident donc la plante à se défendre. Si la défense naturelle des plantes, 
basée sur la biodiversité, pouvait être mobilisée plus efficacement, il y aurait moyen de concevoir des stratégies propres et efficaces de protection des cultures qui réduiraient significativement les effets secondaires négatifs des engrais chimiques de la génération actuelle.

De nombreux bassins hydrographiques très importants sont densément peuplés et ils sont en grande partie voués à l'agriculture ; une grande partie des terres restantes sont une mosaïque agricole où les cultures, l'élevage et la production forestière influencent les systèmes hydrologiques. Dans de telles régions, il est possible de gérer l'agriculture de façon à préserver les fonctions critiques des bassins que sont le maintien de la qualité de l'eau, la régulation du débit, la recharge des nappes aquiferes, la diminution des risques d'inondation, la limitation des flux de sédiments et la préservation des espèces d'eau douce et des écosystèmes. Une gestion de l'eau efficace passe aussi par le choix de combinaisons de cultures qui retiennent l'eau, par la gestion des sols et de l'eau (dont l'irrigation), par des barrières végétales qui ralentissent l'écoulement des eaux le long des pentes, par un couvert végétal permanent et par l'entretien de la végétation naturelle des zones riveraines, des zones humides et des autres zones stratégiques des bassins hydrographiques.

Les paysages agricoles riches en biodiversité, bien gérés, peuvent aussi fournir une protection contre les événements naturels extrêmes. Alors qu'on annonce des pénuries d'eau et la multiplication des phénomènes naturels extrêmes pour les prochaines décennies dans de nombreuses régions du monde, la contribution de la biodiversité au renforcement de la capacité qu'ont les systèmes agricoles de soutenir les fonctions des bassins pourrait être une des principales notions à considérer dans les investissements et la gestion agricoles.

Les paysages agricoles peuvent conserver une vaste gamme d'espèces indigènes, particulièrement celles qui s'adaptent bien à la fragmentation des habitats et à l'utilisation agricole des sols. La probabilité de préserver la biodiversité dans les paysages agricoles dépend du degré de fragmentation et de la connectivité fonctionnelle de ces zones naturelles, de la qualité de leurs habitats, de celle de la matrice productive et de la mesure dans laquelle les agriculteurs veulent préserver la biodiversité en gérant leurs terres. Les modes d'agriculture qui réussissent à équilibrer productivité, meilleurs moyens de subsistance et conservation de la biodiversité à l'échelle d'un paysage sont appelés " éco-agriculture » (McNeely et Scherr, 2003).

Les efforts pour préserver des habitats naturels dans des zones agricoles sont de longue haleine ; ils passent par la mise en réserve de certaines zones, par la rotation des cultures, la mise en jachère de certaines surfaces et la présence d'arbres dans les terres cultivées. On a prouvé que les terres soustraites à la production conventionnelle de cultures augmentaient sans équivoque la biodiversité en Amérique du Nord et en Europe (van Buskirk et Willi, 2004). Pour de nombreuses monocultures commerciales, le fait de laisser incultes des bandes de terre le long des champs pour la protection de l'habitat n'a pas réduit la production totale, parce que les intrants sont répandus de façon plus économique sur le reste (Clay, 2004).

Pourtant, les interventions à l'échelle du paysage conçues spécialement pour protéger les habitats et leur biodiversité sont beaucoup plus efficaces qu'une approche ferme par ferme. Une étude récente réalisée en Amérique du Nord pour savoir quelle proportion d'habitat sauvage est " suffisante » dans des paysages agricoles (Blann, 2006) a conclu que les besoins des habitats devaient être envisagés dans le Cadre de l'historique du paysage et de son contexte. Les îlots d'habitat doivent être assez grands et connectés les uns aux autres, par exemple le 
long des rivières et des ruisseaux ou encore par des pentes abruptes de collines couvertes de végétation indigène. Des îlots plus petits d'habitats naturels peuvent suffire si les parcelles agricoles voisines sont gérées de façon écologique. Des publications toujours plus nombreuses montrent que la connectivité du paysage entre de grands îlots de forêts peut en effet être préservée si l'on conserve une couverture arborée sur l'exploitation agricole, comme des haies vives, des coupe-vent ou des bandes boisées dans les pâturages et dans les champs (Harvey et al., 2004). Les efforts de conservation de la biodiversité destinés à s'adapter aux changements des paysages agricoles devraient dès lors se concentrer sur la protection (ou la restauration) de grandes surfaces d'habitats originels au sein $\mathrm{du}$ maillage agricole, et sur le maintien d'éléments, comme les haies, les arbres isolés, les forêts riveraines et les autres zones non cultivées, qui améliorent

la connectivité du paysage. De telles mesures garantiront l'hétérogénéité au niveau du champ et du paysage et augmenteront donc l'adaptabilité des écosystèmes agricoles face aux changements climatiques, à la demande de cultures nouvelles, à l'évolution de la démographie et à d'autres facteurs dynamiques.

\section{L'AVENIR DE LA BIODIVERSITÉ ET DE L'AGRICULTURE}

Du point de vue de la conservation de la biodiversité sauvage, les systèmes idéaux de production agricole sont ceux qui imitent la structure et la fonction des écosystèmes naturels (Blann, 2006 ; Jackson et Jackson, 2002). Dans des écosystèmes forestiers humides et subhumides, les exploitations agricoles ressembleraient à des forêts, avec des cultures arborées productives, des cultures de plantes ombrophiles en sousétage et de l'agroforesterie. Dans les écosystèmes de prairies, les systèmes de production seraient davantage basés sur des céréales et des plantes

herbacées pérennes, avec des espèces d'arbustes et d'arbres xérophiles économiquement intéressantes. Des cultures annuelles se feraient aussi dans ces systèmes, mais comme cultures intercalaires ou comme des parcelles de monoculture insérées dans des mosaïques de production pérenne et dans des zones d'habitats naturels. La diversité des cultures et des races de bétail domestiquées serait encouragée à l'échelle

de tout un paysage, et la diversité génétique au sein des espèces serait préservée in situ à l'échelle d'un vaste écosystème pour garantir la résilience du système et la diversité écologique nécessaire pour s'adapter aux conditions changeantes.

Les systèmes d'agroforesterie multi-étagée, les coupes à blanc laissées à une repousse naturelle et les jardins sauvages privés sont particulièrement riches en biodiversité sauvage. Par exemple, la hauteur de la canopée, les arbres, les épiphytes, la diversité des espèces d'arbres et de lianes, la complexité de la structure de la végétation, 
le pourcentage de sol recouvert de litière de feuilles, et le taux de calcium, d'azote et de matière organique dans la couche supérieure des sols sont tous significativement plus hauts dans les fermes ombragées que dans celles qui sont exposées au soleil. Par contre, la température de l'air et du sol, la diversité des mauvaises herbes et le pourcentage de sol qu'elles couvrent sont significativement plus grands dans les fermes dépourvues d'arbres. En Amérique centrale, des combinaisons de polycultures et des systèmes de gestion complexes permettent d'augmenter la production de café, de cacao, de bananes, de grumes et d'autres produits d'arbres commerciaux.

Si le café produit dans des monocultures exposées en plein soleil a de meilleurs rendements, le café qui pousse à l'ombre est de loin plus

\section{Encadré 20.1 Cabrucas : préserver les} chauves-souris tout en produisant du cacao

Dans l'Etat de Bahia, au Brésil, les plantations de cacao traditionnelles ombragées (appelées localement cabrucas) sont aussi l'habitat de nombreuses espèces forestières, dont une riche communauté de chauves-souris qui se nourrit de nombreuses espèces d'insectes et qui participe à la pollinisation des espèces végétales qui fleurissent la nuit. Mais lorsque les cabrucas sont situées à plus d'un kilomètre des forêts naturelles, les communautés de chauvessouris sont moins variées que celles qui vivent en forêt. C'est pourquoi il faut considérer l'ensemble du paysage pour en faire la gestion, en tenant compte du fait que l'entretien des cabrucas et le maintien et la restauration des îlots forestiers sont essentiels pour la préservation de la diversité des chauves-souris

Source: Schroth et Harvey, 2007 avantageux pour l'agriculture durable et la conservation de la biodiversité (il accueille souvent plus du double d'espèces d'oiseaux). Les systèmes qui comprennent beaucoup d'espèces d'arbres donnant de l'ombre accueillent de nombreux insectes bénéfiques, des orchidées, des mammiferes et d'autres espèces et ils protègent les fragiles sols tropicaux de l'érosion, ils leur apportent des nutriments et éliminent les mauvaises herbes, ce qui réduit, voire supprime tout besoin d'herbicides et d'engrais chimiques et donc aussi les coûts de production. Les agriculteurs peuvent aussi récolter diverses espèces de fruits, du bois de feu et de charpente et des plantes médicinales.

Pour remplacer les cultures qui doivent être replantées chaque année (d'habitude des monocultures - une seule espèce est semée sur une surface très étendue), des cultures nouvelles et améliorées, comme des fruits, des légumes à feuilles, des épices et des huiles végétales, deviennent de plus en plus populaires. Les cultures pérennes peuvent se montrer plus résilientes et signifient moins de perturbation des sols et des écosystèmes que les cultures annuelles, et elles donnent une plus grande valeur aux habitats, surtout si elles poussent mélangées à d'autres ou en mosaïque (Jackson et Jackson, 2002).

La planification stratégique du développement agricole a commencé à s'intéresser à l'adaptation des systèmes aux changements climatiques, pour anticiper les températures en hausse et les phénomènes naturels plus marqués. Pour chaque augmentation d'un degré Celsius pendant la saison de croissance, les récoltes de riz, de froment et de maïs chutent d'environ $10 \%$ (Brown, 2004). Les cultures de rapport comme le café et le thé, qui ont besoin d'environnements plus frais, seront aussi touchées et pousseront les agriculteurs à aller plus haut sur les collines, et à défricher au fur et à mesure de nouvelles terres. 
Il est probable que des forêts montagnardes importantes pour la biodiversité seront alors de plus en plus menacées. Pour donner des réponses efficaces aux changements climatiques, il faudra changer de variétés, modifier la gestion des sols et de l'eau et mettre au point de nouvelles stratégies pour la gestion des espèces nuisibles étant donné que celles-ci, tout comme leurs prédateurs naturels et leurs cycles vitaux, vont aussi se modifier suite aux changements climatiques. Il est probable qu'en modifiant la diversité à l'échelle des exploitations agricoles et des paysages, nous donnerons une réponse importante en matière de réduction des risques et d'adaptation aux changements climatiques.

Depuis les années 1960, tant l'agriculture industrielle des pays développés que la Révolution verte des pays en développement sont tributaires de semences améliorées, d'engrais et de pesticides chimiques et de l'irrigation. Ce modèle de production implique qu'il n'y a qu'un petit nombre de cultures, plantées généralement en monoculture, pour augmenter la rentabilité en utilisant des intrants externes et la mécanisation. La flore et la faune sauvages y sont considérées comme des concurrentes directes pour les ressources ou les produits récoltés, et l'eau est détournée des zones humides et des habitats naturels pour servir à l'irrigation. Mais au cours des deux dernières décennies, la recherche a montré la valeur de la biodiversité agricole sous toutes ses formes, comme la diversité génétique des cultures et du bétail, les espèces associées importantes pour la production (ex. pollinisateurs, microorganismes du sol, insectes bénéfiques, et prédateurs de nuisibles) et les espèces sauvages qui trouvent leur niche dans des paysages agricoles (Uphoff et al., 2006).

Diverses disciplines, philosophies ou conditions géographiques ont donné naissance à toute une variété d'approches qui favorisent la biodiversité. Ces alternatives à l'agriculture industrielle comprennent l'agroécologie (Altieri, 1995), l'agriculture de conservation (FAO, 2001a), l'agriculture biologique (IFOAM, 2000) et l'agriculture durable (Pretty, 2005). Elles tendent à se concentrer sur la préservation de la base des ressources pour la production, en gérant les cycles de nutriments, en protégeant les pollinisateurs et les microorganismes bénéfiques, en préservant la santé des sols et en économisant l'eau. Elles cherchent à réduire l'empreinte écologique des zones cultivées et les dommages causés à la biodiversité sauvage par les produits chimiques toxiques, la perturbation des sols et la pollution de l'eau. Sous de nombreux aspects, elles ressemblent aux formes de production agricole préindustrielles, mais elles profitent des approches modernes qui augmentent les rendements et la productivité du travail tout en préservant la biodiversité.

La culture biologique aide la biodiversité en utilisant moins de pesticides et d'engrais non organiques et en adoptant une gestion favorable à la faune sauvage des habitats non cultivés, par exemple grâce à des stratégies qui consistent à ne pas faucher près des bordures et à intercaler les terres arables et celles vouées à l'élevage. Ces productions mixtes profitent particulièrement à certaines espèces d'oiseaux, dont celles qui nichent dans les champs. Certaines exploitations qui n'adoptent que quelques pratiques de production biologique, comme le fait de remplacer le désherbage chimique par des méthodes mécaniques, peuvent favoriser la biodiversité autant que les exploitations totalement biologiques.

L'avenir de l'agriculture dépendra beaucoup de la contribution des femmes. Les femmes sont les principales productrices des cultures de base dans le monde (riz, froment, maïs) ; elles produisent jusqu'à $90 \%$ de la nourriture consommée par les pauvres des régions rurales et de $60 \%$ à $80 \%$ de la nourriture dans la plupart des pays en 
développement. En Inde, ce sont les femmes qui assurent $75 \%$ du travail de plantation et de désherbage du riz, $60 \%$ encore pour la récolte et $33 \%$ pour le battage (Communiqués de presse du Centre d'informations des Nations Unies de Sydney, pour l'Australie, la Nouvelle-Zélande et le Pacifique Sud, 1995, cités dans Mata \& Sasvari, 2009).

\section{Selon l'Organisation des Nations Unies pour} l'alimentation et l'agriculture (FAO), les femmes produisent, sélectionnent et économisent jusqu'à 90\% des semences et des germoplasmes utilisés pour les plantations dans les petites exploitations. $\mathrm{Au}$ Rwanda, les femmes produisent plus de 600 variétés de haricots, et les femmes Aguaruna du Pérou cultivent plus de 60 variétés de manioc (FAO, 2001b).

Selon la Stratégie et le Plan d'action nationaux pour la biodiversité (NBSAP) au Yémen, les femmes ont aussi un rôle clé en conservant et en plantant des espèces sous-utilisées, qui ne répondent pas à une large proportion des besoins alimentaires mondiaux mais qui sont utilisées par des communautés particulières pour compléter leur régime alimentaire. Au Yémen, les femmes font pousser des cultures différentes de celles des hommes, les "cultures de femmes", comme des arachides, des potirons, des légumes à feuilles, des doliques, des concombres et des patates douces, ce qui a pour effet d'augmenter la biodiversité agricole et la sécurité alimentaire (NBSAP Yémen, 2005). Le NBSAP du Bhutan reconnaît aussi que des espèces sous-utilisées contribuent considérablement à l'alimentation des foyers et à la sécurité alimentaire ; elles sont souvent cultivées ou récoltées par des femmes. La connaissance des utilisations et de la gestion de ces espèces est également très localisée et spécialisée (NBSAP Bhutan, 2002).

Au cours de la prochaine décennie, la communauté de la conservation, en travaillant en plus étroite collaboration avec les organisations agricoles, devrait rechercher des modes durables et adaptables d'utilisation des terres, qui attribuent une grande priorité à la préservation des espèces sauvages apparentées aux plantes et aux animaux domestiques (notons que beaucoup de ces espèces sont menacées). Pour intégrer des modes d'agriculture compatibles dans les stratégies et les plans d'action de conservation de la biodiversité à l'échelle des paysages, il faudra renforcer l'expertise des agriculteurs en tant que gestionnaires de l'environnement et faire connaître les multiples valeurs de la biodiversité pour l'agriculture, ce qui aidera à renforcer le soutien de la conservation.

La biodiversité et les services écosystémiques devraient être intégrés dans les recherches et le développement agronomiques pour s'assurer que les nouvelles technologies agricoles appuieront la conservation de la biodiversité au lieu de la menacer. Enfin, le développement de nouvelles approches pour rémunérer les agriculteurs qui contribuent à la conservation de la biodiversité et au maintien des services écosystémiques permettra de fournir les incitants nécessaires pour renforcer les quêtes communes de la conservation et de l'agriculture.

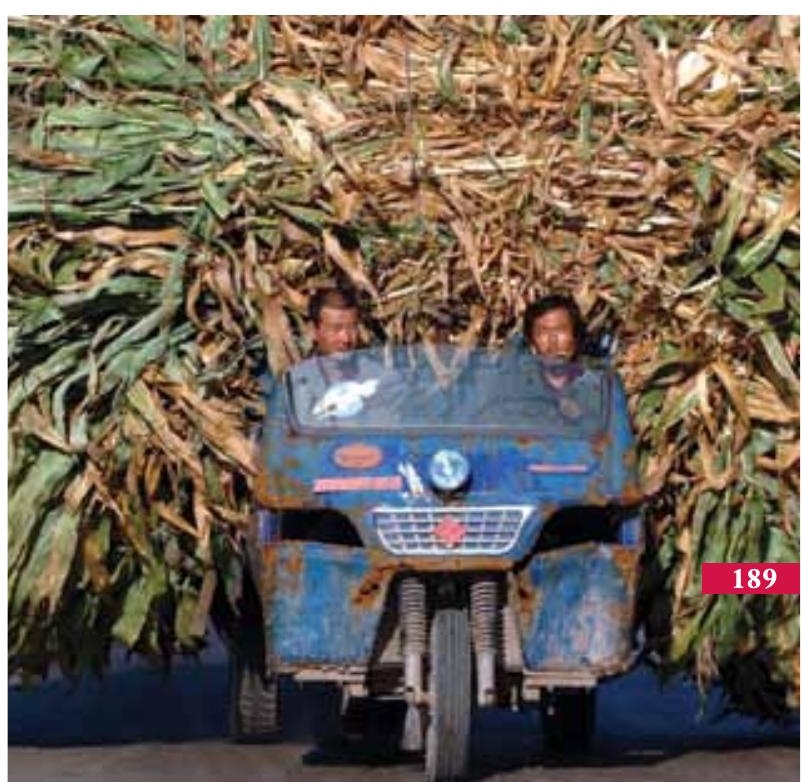




\section{Systèmes urbains : \\ 21. la conservation dans la ville}




\section{En 1900, 160 millions de personnes environ vivaient en ville, soit à}

peu près $10 \%$ de la population mondiale qui était d'1,6 milliard à cette époque. En 2000, près de la moitié des six milliards de personnes vivaient dans des zones urbaines, et ce pourcentage continue de progresser (Chapitre 1). Si la conservation se veut un phénomène mondial, il faut trouver de nouvelles voies pour permettre aux gens qui vivent dans les villes d'y participer activement.

La conservation de la nature dans les villes peut être abordée de différentes façons. Benton-Short et Short (2007) apportent une perspective sociale tandis que Shiro (2004) adopte une approche de la planification qui considère les villes plutôt comme de gigantesques organismes et qu'Isenberg (2006) apporte un point de vue plus historique, avec des exemples venus des États-Unis, d'Europe et d'Afrique.

Si la conversion de forêts et de terres agricoles en villes cause inévitablement une perte de la biodiversité, les habitants des villes, eux, font un usage moins important de certaines ressources que ceux qui vivent à la campagne. Les appartements des hauts buildings sont plus économes en énergie que les maisons individuelles, et les villes ont des moyens de production d'eau, d'énergie et de transports globalement plus rentables que les régions rurales. À Londres, les émissions de dioxyde de carbone par personne sont seulement légèrement supérieures à la moitié de la moyenne nationale, et les habitants de New-York produisent moins du tiers des émissions moyennes, par personne, du pays tout entier. São Paulo et Rio de Janeiro ont, elles aussi, réduit substantiellement leurs émissions de dioxyde de carbone par personne, mais des villes comme Beijing et Shanghai, qui comprennent de nombreuses usines à fortes émissions, se classent bien au-dessus des moyennes nationales. Au niveau mondial, d'après les chiffres d'ONU-Habitat, les villes émettent $50-60 \%$ des gaz à effet de serre (GES), et ces chiffres passent à près de $80 \%$ si l'on y ajoute les émissions indirectes.

Bien sûr, les villes posent des problèmes pour les écosystèmes. Elles occupent $2 \%$ de la surface terrestre mais consomment $75 \%$ des ressources naturelles. Quand plus de personnes s'entassent sur des superficies plus réduites, les maladies infectieuses risquent de se transmettre plus facilement. La biodiversité des villes, surtout au niveau des écosystèmes et des espèces, est particulièrement menacée par des espèces exotiques envahissantes. Ceci n'est pas étonnant dans la mesure où les villes sont souvent les centres du commerce international qui transporte avec lui des espèces envahissantes (Schwartz et al., 2006). Cela s'étend aussi aux oiseaux, et l'avifaune des zones urbaines a tendance à s'homogénéiser de plus en 
plus, les espèces rares allant vers une disparition complète et les espèces cosmopolites comme les pigeons ou les moineaux devenant dominantes (Clergeau et al., 2006). Et les gens qui vivent en ville doivent puiser dans les campagnes environnantes pour une grande partie de leurs ressources essentielles, particulièrement la nourriture, l'eau et l'énergie.

Ils ont aussi besoin des aires protégées, qui procurent d'importants avantages aux villes, comme l'apport d'eau, les loisirs et diverses valeurs économiques. De nombreuses personnes vivant dans les villes attendent des aires protégées qu'elles leur procurent un bien-être psychologique certain, et elles trouvent qu'une semaine passée dans la nature sauvage d'un parc national est un répit revigorant et indispensable dans les pressions que les villes surpeuplées et impersonnelles font peser sur leur vie. Les aires protégées aussi dépendent des villes, pour le soutien politique, les visiteurs, et le lien culturel qu'elles maintiennent entre les gens des villes et leur environnement.

De nombreuses organisations de conservation reconnaissent qu'il est important d'intégrer des espaces naturels au sein de l'infrastructure des grandes villes. Cela va bien au-delà des simples parcs de quartier même si ceux-ci jouent évidemment un rôle important. Certaines villes se sont montrées ambitieuses dans leur volonté d'intégrer la biodiversité dans la planification urbaine. Londres, par exemple, a adopté une Stratégie officielle pour la biodiversité qui comprend cinq éléments majeurs : permettre à ceux qui vivent ou qui travaillent à Londres d'avoir un meilleur contact avec la nature dans leur propre ville ; protéger les habitats importants pour la vie sauvage de Londres et identifier plus de 1500 de ces sites ; améliorer les habitats dans les parcs publics et des espaces ouverts ou créer de nouveaux habitats sauvages pour le plaisir du public et l'éducation environnementale ; favoriser la création d'installations pour l'éducation environnementale et offrir des opportunités pour que tous les secteurs de la société puissent s'impliquer activement dans des projets environnementaux ; et attirer une grande diversité d'organisations et de particuliers dans un partenariat en faveur de la Stratégie pour la biodiversité (Goode, 2005). De nombreuses villes ont des parcs nationaux à l'intérieur de leurs limites, ou juste à côté, y compris de grandes métropoles comme Los Angeles, Rio de Janeiro et Sydney. Les aires protégées situées à l'intérieur des villes aident à protéger les ressources en eau, offrent des possibilités de loisirs, aident à encourager l'éducation environnementale et créent des emplois locaux.

L'UICN cherche à coordonner les actions de conservation dans les villes, entraînée par le Groupe de travail de la Commission mondiale des aires protégées (CMAP) sur les villes et les aires protégées, qui lui-même a contribué au Partenariat mondial pour les villes et la biodiversité qui a commencé en 2006. Et plus de 300 gouvernements locaux ont rejoint un réseau qui appuie le Compte à rebours 2010 de l'UICN, qui cherche à inverser le rythme de perte de la biodiversité à cette date.

\section{LES VALEURS DE LA NATURE DANS LES VILLES}

On peut s'attendre à ce que les zones urbaines apportent un plus grand support aux aires protégées lorsque les gens qui y vivent reconnaissent les bienfaits que ces aires leur procurent. Par exemple, Dudley et Stolton (2005) ont découvert que près d'un tiers (33 sur 105) des plus grandes villes du monde recevaient leur eau potable directement d'aires protégées ; c'est le cas de Barcelone, Bogota, Brasilia, Caracas, Jakarta, Johannesburg, Karachi, Los Angeles, Madrid, Melbourne, Mumbai, Nairobi, New York, Perth, Rio de Janeiro, São Paulo, Singapour, Sydney, Tokyo et Vienne.

De nombreuses autres villes gèrent des forêts spécifiquement pour la protection des bassins 
hydrographiques ; citons Séoul, Tokyo, Beijing,

Rangoon, Santiago, New York, Stockholm,

Munich et Minsk. Quelque 90\% de l'eau fournie

à Melbourne (Australie) proviennent de captages

situés dans des forêts de montagne inhabitées,

situées au nord et à l'est de la ville. La majorité de

ces zones de captage se trouvent en dehors d'aires

protégées, mais elles sont gérées par la société

parastatale Melbourne Water pour protéger ces

captages forestiers. Lorsque l'on établit le lien entre

la contribution très pratique des aires protégées ou des autres zones riches en biodiversité et les villes, cela permet de construire un support plus solide en leur faveur.

Le fait de pouvoir accéder à des espaces verts en ville est très bénéfique pour les gens, spécialement pour leur santé, leur sécurité et leur bien-être (Kuo et al., 1998). Fuller et Gaston (2009) ont voulu évaluer les espaces verts de 386 villes dans 31 pays européens comptant plus de 170 millions de personnes (plus d'un tiers de la population européenne). Ils ont découvert de grandes disparités, avec 1,9\% d'espaces verts à Reggio di Calabria, en Italie, comparé à 46\% à Ferrol, en Espagne ; les villes du nord de l'Europe ont en général une plus grande proportion d'espaces verts que celles du sud. Sans surprise, ils ont aussi trouvé que la proportion d'espace vert par personne diminue généralement lorsque la densité de population augmente. La densité croissante de la population des villes est certainement une menace pour les espaces verts restants. Par exemple, Mexico perd chaque année 500 hectares de parc et de zones forestières à cause des squatters et de développements divers qui, ensemble, ont absorbé en dix ans près de la moitié des aires protégées ouvertes qui restaient dans la capitale.

Certains experts considèrent que les combustibles fossiles sont indispensables pour l'approche moderne de la vie urbaine (Girardet, 1999). Jusqu’à très récemment, la plupart des villes avaient ce que
Encadré 21.1 Des oiseaux dans la ville

Les oiseaux des paysages urbains occupent d'abord des parcs (qui peuvent être vus comme des fragments de forêts), des rues arborées (des bandes linéaires qui relient les fragments) ou la matrice urbaine ; les pigeons, par exemple, voient les bâtiments comme leurs ancêtres voyaient des falaises, et des faucons habitent à Manhattan où les pigeons constituent des proies abondantes. A Madrid, en Espagne, FernandezJuricic (2000) a étudié les effets de l'emplacement des rues dans le paysage, de la structure de la végétation et des perturbations humaines (quantité de piétons et de voitures) dans les rues arborées, sur la richesse en espèces d'oiseaux, leur permanence dans le temps, la densité de guildes de nourrissage et de nidification et la probabilité de l'occupation de certaines rues par des espèces particulières. Le nombre d'espèces augmentait des habitats les moins favorables (rues sans végétation) jusqu'aux plus favorables (parcs urbains), les rues arborées constituant des éléments intermédiaires du paysage. Les rues bordées d'arbres qui connectent les parcs urbains influençaient positivement le nombre d'espèces dans les rues, la persistance des espèces, la densité des guildes et la probabilité que les rues soient occupées par des espèces particulières. Les perturbations humaines exerçaient une influence négative sur les mêmes variables. Les rues arborées pourraient fonctionner comme des corridors qui permettent à certaines espèces de prospérer en maintenant un habitat qui leur permet de se nourrir, de nicher et de se reposer (pour les espèces migratrices). Des améliorations locales de la qualité des corridors, en augmentant la variété de la végétation et en réduisant les perturbations humaines, pourraient avoir un effet positif sur la connectivité régionale du système et sur les qualités qui en font un habitat adapté aux oiseaux.

Source : Fernandez-Juricic et Jokimaki, 2001 
l'on peut considérer comme un métabolisme linéaire, avec des ressources qui affluaient dans le système sans que les consommateurs se soucient ni de leur origine, ni du sort de leurs déchets. Un modèle plus approprié pour les villes serait d'imiter le métabolisme circulaire de la nature, où chaque extrant est aussi un intrant qui aide à alimenter et à renouveler l'ensemble du système - l'essence même du service écosystémique. Le recyclage est déjà devenu un comportement normal dans de nombreuses villes, et la crise financière actuelle a montré qu'il était

\section{Encadré 21.2 Chicago Wilderness}

\section{Conçue sur le modèle de l'UICN, Chicago}

Wilderness est une alliance participative régionale qui est très engagée dans la conservation de la biodiversité dans la région métropolitaine de Chicago et veut relier des hommes à la nature. Plus de 240 organisations membres très diverses travaillent ensemble dans des programmes et des recherches scientifiques dans le but de protéger et de restaurer les zones naturelles dans une région urbaine qui s'étend sur quatre états. L'alliance cherche à augmenter la sensibilisation et la connaissance de la biodiversité originelle et des valeurs de la nature de la région ; à accroître et à diversifier la participation dans la gestion environnementale; à construire des relations participatives parmi les diverses circonscriptions de la région pour susciter une relation durable avec la nature ; à faciliter la mise en application des recherches en sciences naturelles et en sciences sociales dans la restauration et l'entretien des divers environnements de la région; à encourager le développement des meilleures pratiques de gestion et le partage des informations ; et à assurer un vaste soutien, public et privé, à son objectif qui est de voir préservée la biodiversité indigène - l'espace sauvage - dans la région métropolitaine de Chicago et, dans la foulée, de reconnecter de nombreuses tout à fait possible de vivre une vie urbaine décente sans ces fortes consommations de ressources. Une ville durable sera capable de répondre à ses propres besoins sans menacer le monde naturel ni les conditions de vie de ses citoyens.

\section{UNE NOUVELLE VISION DES VILLES ET DE LA NATURE}

De nombreuses métropoles sont déjà relativement vertes : Beijing, par exemple, produit déjà dans la région presque tous les légumes qu'elle consomme. D'autres veulent devenir plus vertes.

personnes avec la nature de la région et avec les derniers endroits sauvages.

Le nom Chicago Wilderness s'applique aussi à la mosaïque régionale d'aires naturelles dispersées sur plus de 145.000 hectares d'étendues de terres et d'eau protégées. Les communautés naturelles de Chicago Wilderness comprennent des prairies de hautes herbes, des forêts de feuillus, des savanes à chênes, des prairies à carex, des marais, des tourbières et des fagnes. Il reste moins d'un dixième de pour cent des prairies de hautes herbes originelles en Illinois, mais Chicago Wilderness en a quelques-uns des meilleurs exemples, qui contiennent plusieurs espèces menacées à l'échelle régionale.

L'alliance Chicago Wilderness a été formée en 1996 pour améliorer la gestion des nombreuses aires protégées qui entourent Chicago et qui vont du sud-ouest du Michigan au sud-est du Wisconsin en passant par le nord-ouest de l'Indiana et le nord-est de l'Illinois. L'alliance a d'abord produit un Atlas de la biodiversité, puis un Plan de restauration de la biodiversité, et ensuite un Bulletin sur le statut des biotes et des écosystèmes de la région. Elle produit aussi un magazine trimestriel et elle a publié un guide familial pour encourager les activités de jeunes dans la nature. Le magazine contient des articles sur des espèces natives 
Malgré certains efforts considérables pour "verdir " les villes, la plupart des villes du monde sont concentrées au sein d'une biodiversité appauvrie (Turner et al., 2004). Des milliards de personnes risquent de perdre la possibilité d'apprécier la nature, et de ne pas pouvoir profiter des bienfaits qu'elles pourraient en retirer. Ceci veut dire que ça vaut la peine - que c'est même indispensable - de déployer des efforts vigoureux pour donner aux gens des possibilités de renouer des liens avec la biodiversité à l'intérieur des villes.

particulières, rend honneur aux professionnels et aux particuliers actifs dans la conservation et décrit toute une variété d'endroits protégés de la région. Il y a eu aussi des numéros spéciaux sur des sujets importants comme les ressources en eau et la construction de routes. Pour ce qui est des membres de l'alliance Chicago Wilderness, il $\mathrm{y}$ a des agences qui relèvent des gouvernements fédéral, de l'Etat ou locaux, des municipalités et des districts de parc, de grandes organisations non gouvernementales de conservation, de petits groupes de bénévoles, des organisations d'éducation et de recherche et des institutions culturelles. Un Conseil d'entreprise a été constitué où 35 sociétés ont promis leur soutien et leur participation aux efforts de l'alliance. Le support financier vient maintenant des organisations membres et du Conseil d'entreprise, de sources philanthropiques et des abonnés au magazine, mais une grande partie du financement de la programmation est venue de deux agences fédérales, le Fish and Wildlife Service et le US Forest Service, qui constituent les défis de contrepartie pour les autres organisations membres.

\section{Le calendrier de l'alliance Chicago Wilderness a} aujourd'hui quatre pôles : un groupe de travail sur les effets des changements climatiques sur la biodiversité régionale, un plan visionnaire pour
Certaines villes en ont déjà conscience. Jinan, la capitale de la province orientale du Shandong, va planter 7100 hectares de nouvelles forêts dans les trois ans qui viennent. La ville prévoit de donner à toute personne de 11 ans et plus la responsabilité de planter de trois à cinq arbres par an, dans le Cadre du Projet Ciel bleu de la ville qui veut, dans les cinq ans, créer un environnement propre et vert dans cette ville plutôt polluée.

Un programme novateur souhaite relier la jeunesse urbaine à la nature dans la campagne : c'est le

le paysage qui veut quintupler les aires protégées pour la pérennisation des divers biotes indigènes, le programme Leave no Child Inside (" ne laissez aucun enfant à l'intérieur ») qui veut développer l'intérêt des enfants pour la nature et construire des capacités de gestion de l'environnement et enfin une initiative à long terme pour approfondir les connaissances en matière d'écologie de la restauration et les appliquer à la gestion.

Chicago Wilderness marche parce qu'elle s'est construite sur un siècle d'histoire de la conservation locale, qu'elle a un groupe varié de fondateurs composé de leaders d'organisations déjà dévoués à la cause de la conservation de la biodiversité, qu'elle est très visible auprès du public grâce à ses publications et qu'elle a permis à ses membres de mieux s'acquitter des missions de leurs propres organisations. Même lorsque ces conditions sont absentes, Chicago Wilderness offre un modèle marquant qui montre comment une approche participative de la conservation de la biodiversité peut se mettre en place dans un environnement urbain, et ce modèle a déjà été repris à Curitiba, au Brésil, et à Houston, au Texas. 
programme connu sous le nom de « Les Enfants pour les Tigres ", qui a été lancé en Inde en 2001, avec pour objectif d'encourager les enfants des villes de toute l'Asie du Sud de visiter la nature. Même si les tigres en sont l'emblème, beaucoup des sujets les plus importants concernaient l'eau (Sahgal, 2005). Plus d'un million d'enfants ont pris part au programme, venant de 700 écoles dans 12 villes.

Ces dernières années, l'« écologie urbaine » a évolué et est devenue une initiative pour intégrer les sciences naturelles et sociales à l'étude des environnements des villes et de leurs effets régionaux et mondiaux, en se fondant sur le principe que les villes apportent les problèmes et les solutions aux défis de la durabilité dans un monde de plus en plus urbanisé (Grimm et al., 2008). La Chicago Wilderness Area en est un exemple exceptionnel (Encadré 21.2).

Les plantes envahissantes deviennent souvent plus nombreuses dans les zones urbaines, mais cela peut augmenter la richesse en espèces des villes par rapport aux zones rurales, et même par rapport aux aires protégées. Les villes se caractérisent par un patchwork d'habitats très hétérogènes, et les gens introduisent des espèces végétales non indigènes, quelques individus seulement par espèce, dans les jardins de ville.

De nombreuses villes possèdent aussi des zoos et des jardins botaniques qui accueillent certes des espèces sauvages indigènes, mais qui donnent aussi aux gens de la ville la possibilité d'entrer en contact avec des espèces végétales et animales du monde entier.

Les écosystèmes urbains ressemblent souvent peu aux écosystèmes ruraux ; les communautés d'oiseaux passent souvent des espèces granivores à des espèces insectivores, et de nombreuses communautés d'insectes perdent souvent leurs espèces spécialisées au profit d'espèces plus généralistes. De nombreuses villes ont tendance à avoir des espèces adaptées à la ville qui sont semblables, ce qui entraîne une homogénéisation et non une diversité (Grimm et al., 2008). Et comme les villes se caractérisent par des températures généralement plus élevées et par une plus forte luminosité nocturne, de nombreuses espèces nocturnes sont comparativement désavantagées. Grimm et ses collègues prônent une « écologie de la réconciliation » où les habitats qui sont fortement modifiés pour l'usage humain sont conçus, spatialement aménagés et gérés de façon à optimiser la biodiversité tout en fournissant des bénéfices et des services écosystémiques. Ils laissent entendre que l'écologie de la réconciliation pourrait offrir aux écologistes des opportunités intéressantes de contribuer à la conception et à la gestion de villes nouvelles et d'aider à reconstruire les plus anciennes.

Comme les communautés biologiques des villes sont celles que connaît normalement la moitié de la population humaine, il est de plus en plus important de tirer au maximum profit des derniers restes de " nature » qui subsistent dans les zones urbaines pour susciter un plus vaste support de la conservation. Les habitants des villes doivent encourager et voter pour des stratégies urbaines qui sont plus économiques en ressources, défendre des programmes d'éducation sur la nature des centres urbains et favoriser une culture de durabilité et de conservation urbaines.

Il faudrait encourager les décideurs urbains à s'engager davantage dans les questions de biodiversité et d'aires protégées et à les inclure dans les réunions pertinentes, qu'elles soient nationales ou internationales. La Conférence des Maires qui aura lieu à Nagoya, au Japon, en 2010 serait une bonne occasion parce qu'elle se tiendra parallèlement à la Conférence des Parties à la Convention sur la diversité biologique (COP CDB). Ils devraient aussi créer des Aires de conservation municipales et reconnaitre qu'elles sont une contribution importante au réseau mondial des aires protégées et à l'effort international pour conserver 
la biodiversité. Ils devraient encore chercher à intégrer des composantes « biodiversité et aires protégées " dans la planification des événements sportifs majeurs qui se passent en ville, comme les Jeux olympiques (d'hiver et d'été) et les Coupes du monde. Enfin, le fait de se rattacher au Centre des
Nations Unies pour les établissements humains et à d'autres parties concernées pourrait améliorer le flux d'informations entre administrateurs urbains et chefs d'entreprises à propos des questions environnementales qui concernent les villes.

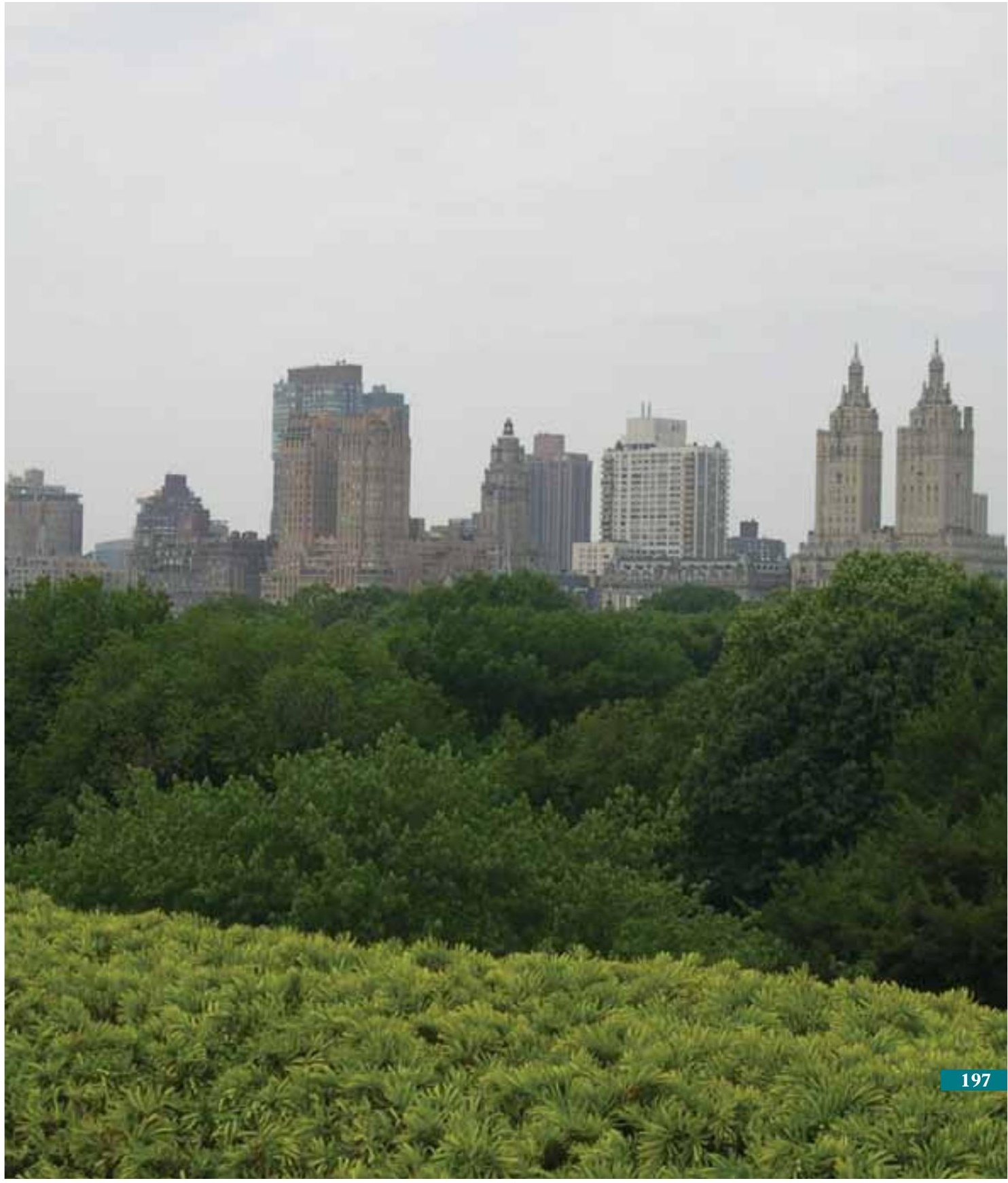


Une feuille de route pour Conservation pour une ère nouvelle 
Ce livre, et le Forum mondial de la nature qui l'a inspiré, a mis en évidence de nombreuses questions et préoccupations mais aussi des opportunités. Nous nous réjouissons de l'émergence d'approches éthiques inclusives de la conservation de la biodiversité dans son ensemble. Nous reconnaissons la première place qu'occupent les changements climatiques dans les menaces mais nous remarquons aussi que d' " anciens » problèmes, comme la dégradation des habitats, la surexploitation et les espèces envahissantes, n'ont pas disparu et qu'ils se sont au contraire imbriqués dans les changements climatiques. Nous voyons les opportunités pour la biodiversité d'aider à résoudre certains des problèmes humains les plus urgents. Ce livre confirme que la nature est importante non seulement pour elle-même, mais aussi pour le bien-être humain.

Alors que des approches potentielles étaient discutées tout au long de ce volume, plusieurs points communs ont émergé. La conservation d'aujourd'hui devra veiller à la spécificité des différents problèmes, mais quelques points clés concernent tout le monde - la feuille de route de l'avenir de la conservation. Il s'agit de :

1. Intégrer la biodiversité et les services écosystémiques à tous les secteurs

2. S'adapter aux changements grâce à la biodiversité, à la créativité et au respect de la nature

3. Promouvoir l'intégration à part entière des politiques qui favorisent l'équité et les droits dans la conservation.

\section{METTRE LA BIODIVERSITÉ AU CENTRE DE} TOUS LES SECTEURS

Le paradigme des services écosystémiques cristallise l'interdépendance de nos vies et de notre environnement. Il nous donne aussi des moyens pour mesurer et contrôler l'impact de nos actions et pour établir plus facilement quels sont les coûts et bénéfices de ces actions. Les services écosystémiques nous offrent aussi un angle d'entrée dans des domaines apparemment pas environnementaux, et le plus évident d'entre eux a sans doute été l'effort pour faire de l'environnement une partie intégrante du développement.

Une étape importante consistera à harmoniser le langage utilisé dans les diverses disciplines. La Commission mondiale des aires protégées (CMAP) de l'UICN reconnaît ce besoin de " parler un langage commun " pour atteindre un large public et l'aider à comprendre le système de classification des aires protégées de l'UICN (Bishop et al., 2004). On a identifié le recours au jargon médical comme un facteur qui peut nuire à la santé du patient parce que 
celui-ci ne comprend tout simplement pas ce que le médecin lui dit (Zeng et Tse, 2006). Si les conservationnistes veulent réellement voir le monde changer en mieux dans les prochaines décennies, ils vont devoir toucher un nouveau public et lui parler dans un langage qu'il comprend bien. Les chapitres sur l'énergie (Chapitre 8), les conflits armés (Chapitre 9), les catastrophes (Chapitre 10), la santé humaine (Chapitre 11), les technologies (Chapitre 13), le secteur privé (Chapitre 15), l'agriculture (Chapitre 20) et les villes (Chapitre 21) vont tous dans ce sens. Parler de services écosystémiques au lieu de biodiversité est un autre pas dans cette direction, et l'Économie des écosystèmes et de la biodiversité (TEEB) se fonde là-dessus pour réunir la communauté économique et celle de la biodiversité. Nous devrons aussi nous servir des nouveaux outils de communication et de sensibilisation qui sont à notre disposition. Ces temps sont révolus, quand les connaissances ne se transmettaient que par des interactions personnelles ou par des livres. Wiki, blogs, e-cours en ligne au lieu des salles de classe, qui sait ce que l'avenir nous réserve pour le partage des sciences de la conservation?

Les gouvernements se rendent toujours mieux compte du rôle que la nature peut jouer dans la réduction de la pauvreté et le développement ; ils ont ainsi la preuve dont ils avaient besoin pour soutenir les investissements dans la nature, moyen fondamental pour favoriser le développement durable dans tous les secteurs. De plus, de nombreux bailleurs de fonds prennent des dispositions pour intégrer l'environnement comme une question interdisciplinaire centrale. Par ce " recentrage", on entend « les processus par lesquels les points de vue environnementaux sont présentés à l'attention des organisations et des particuliers impliqués dans les décisions en matière de développement économique, social et physique d'un pays (au niveau national, sous-national et/ou local), et les processus qui déterminent comment l'environnement sera perçu lors de ces prises de décisions " (IIED, 2009). Englober l'environnement dans le développement exige de s'assurer que les pays récipiendaires incluent réellement l'environnement dans leurs requêtes et que les pays donateurs s'assurent que l'environnement est inclus dans leurs projets. Les gouvernements qui souhaitent un meilleur avenir examinent maintenant le rôle de l'environnement dans leur planification nationale et ils décident qu'il doit compter (Encadré 22.1). Comme le rôle de la biodiversité dans ces processus est fondamental, le recentrage est une question de biodiversité.

\section{Mais cette intégration, vue comme vecteur} de durabilité, influence aussi le secteur privé. Les questions environnementales, qui étaient auparavant considérées comme n'ayant rien à voir avec l'activité économique, sont aujourd'hui en train de réécrire les règles pour les entreprises, les investisseurs et les consommateurs. Les sociétés qui prennent la durabilité au sérieux ont été plus performantes que les autres lors de la récente crise économique (AT Kearney, 2009).

La durabilité à plus long terme exigera cependant que l'environnement soit intégré partout, et particulièrement dans le mode de vie de chacun. Les choix que nous faisons - de la nourriture que nous mangeons aux voitures que nous conduisons et à la façon dont nous nous relaxons - ont tous un impact sur la nature. Puisque nous devenons conscients de la nature de ces impacts, c'est à nous d'assumer la responsabilité de nos actes et de nous joindre aux gouvernements et aux industries dans un effort mondial pour favoriser un environnement plus sain et plus productif.

Pour un avenir durable, il faut aussi soutenir les efforts d'intégration de l'environnement au niveau institutionnel et gérer les comportements individuels. Ce livre donne de nombreux exemples d'actions que tout le monde peut entreprendre pour intégrer l'environnement dans sa propre vie. En voici quelques-uns : 
1. Avoir un mode de vie dont le bilan carbone est neutre, grâce à des choix énergétiques responsables.

2. Vérifier son empreinte hydrique et bien gérer sa consommation d'eau à la maison.

3. Consommer d'une manière favorable à

l'environnement - soutenir les programmes de certification, et suivre les $3 \mathrm{R}$ : réduire, recycler, réutiliser.

\section{Soutenir et voter pour des politiques} gouvernementales qui favorisent la conservation de l'environnement.

\section{S'ADAPTER AUX CHANGEMENTS}

Comme nous l'avons dit dans ce livre, la société humaine se développe aujourd'hui plus vite qu'à toute autre époque et elle est sans cesse interpellée par la portée et les conséquences des changements sociaux, économiques et environnementaux. Pour aborder les défis de la conservation dans les prochaines décennies, une constante doit rester dans nos esprits et dans notre planification - le besoin de faire face à des changements continus.

La population humaine continue à grandir et elle se concentre dans les villes. Les impacts sur la capacité des ressources naturelles de fournir de la nourriture, des fibres et des combustibles sont de plus en plus évidents. La pêche s'effondre dans le monde entier, les forêts disparaissent, et ce sont maintenant les besoins énergétiques qui, tout autant que les besoins alimentaires, déterminent les choix en matière d'agriculture. Les zones urbaines en croissance rapide entraînent une augmentation soutenue, mais peut-être pas soutenable, du commerce de grumes, des produits agricoles, de l'élevage et des exploitations minières qui, à leur tour, aboutissent à des déforestations et à des modifications de l'aménagement du territoire. Et comme les migrations seront peut-être une stratégie importante dans la planification des adaptations
Encadré 22.1 Mise à jour de la PSRP en Tanzanie : intégration de l'environnement au niveau national pour réduire la pauvreté

En Tanzanie, la nouvelle Stratégie nationale pour la croissance et la réduction de la pauvreté (NGSRP) ou Mkukuta représente une nouvelle approche, plus complète, de la réduction de la pauvreté. Elle accorde en particulier plus d'attention aux questions transsectorielles telles que la durabilité environnementale qui contribue à la réduction de la pauvreté et à la croissance. Ceci résulte du fait que le Gouvernement tanzanien s'est rendu compte, tout comme les parties prenantes nationales et les partenaires en développement, que la première PRSP n'avait pas réussi à traiter correctement les questions d'environnement et d'autres questions majeures, qui étaient essentielles pour réduire de façon durable la pauvreté et pour obtenir de la croissance. Quinze des 108 cibles de la NSRGP inscrites dans la Mkukuta sont directement relatives à l'environnement et aux ressources naturelles, et l'on s'attend à ce que des interventions sur l'environnement contribuent aux autres cibles. L'action sur l'environnement devrait permettre d'atteindre les objectifs en matière de gouvernance et de responsabilité. Un élément clé de l'examen qui a mené à la NSGRP fut la responsabilité nationale. Elle fut coordonnée en dehors du bureau du vice-président et elle fut le résultat de la consultation très détaillée d'un très grand nombre de parties prenantes au sujet de son contenu et de ses centres d'intérêt.

Source : PNUE, 2008b, The Environment Times, http://wwew. grida.no/publications/et/ep4/page/2641.asp $x$

aux changements climatiques, spécialement pour ceux qui vivent le long des côtes, il sera critique de bien gérer ces mouvements de populations et leurs impacts. 
Mais ce ne sont pas seulement les services d'approvisionnement qui seront atteints. Les services de soutien et de régulation, qui garantissent des conditions optimales pour la santé humaine, sont aussi en danger. Des preuves flagrantes pointent du doigt les changements démographiques humains comme le facteur contribuant le plus, directement et indirectement, à l'augmentation des maladies infectieuses (Chapitre 11).

Notre monde en évolution rapide a aussi un effet profond sur les services culturels liés à la nature. La culture humaine est inextricablement liée à l'environnement dans lequel nous vivons, et les défis qui touchent notre environnement menacent aussi les cultures du monde entier. Les spécialistes ont estimé que $60-90 \%$ des 6900 langues parlées aujourd'hui dans le monde pourraient disparaître d'ici un siècle (Romaine, 2007), un taux d'extinction qui serait même plus élevé que celui qui est donné dans la Liste rouge de l'UICN des espèces menacées, tous taxons supérieurs confondus. Perdre une langue signifie que l'on perd aussi les savoirs et les pratiques qui y sont liés, dont certains pourraient être vitaux pour notre avenir car ils nous permettraient peut-être de nous adapter aux changements climatiques. Dans le même ordre d'idées, perdre de la biodiversité signifie que l'on perd ce qui constitue le fondement de nombreuses croyances et pratiques traditionnelles.

Tous les services sont aussi affectés tout spécialement par les changements environnementaux qui résultent des changements climatiques. Rien qu'un exemple cité à plusieurs reprises dans ce livre : les espèces exotiques envahissantes, déjà reconnues comme une des raisons majeures de pertes biotiques et économiques, sont en soi des espèces qui s'adaptent bien aux changements et qui vont probablement se répandre davantage et avoir toujours plus d'impacts.

Pour gérer les impacts des changements, nous devrons adopter une double approche - atténuation et adaptation - et cette approche sera aussi applicable aux autres aspects des changements que nous connaîtrons.

Nous devrons, autant que possible, chercher à atténuer les impacts des changements sur l'environnement - que ce soit en réduisant les émissions de gaz à effet de serre (GES), en gérant les migrations de populations ou en passant à des sources mixtes d'énergie. Mais nous devons aussi reconnaître que les impacts des changements sont déjà là, et qu'en raison des délais avant que des impacts soient constatés, il est probable que nous observerons des effets plus graves avant que les mesures d'atténuation aient pu produire leurs effets. C'est pourquoi l'adaptation est aussi nécessaire que l'atténuation. Et cette adaptation doit être dynamique, puisque nous vivons dans un environnement en adaptation constante, pas dans un environnement statique.

Idéalement, toute planification devrait se fonder sur des processus et non sur un état; c'est pourquoi une planification basée sur la fourniture de services écosystémiques offre un modèle utile qui favorise la gestion adaptative. La gestion adaptative et le suivi sont des éléments essentiels dans une telle approche. La gestion adaptative est une approche de gestion qui intègre un suivi régulier et qui actualise les plans et les stratégies en fonction des résultats de ce suivi. C'est un moyen de s'assurer que toute utilisation des ressources est durable et c'est aussi un mécanisme important pour gérer les incertitudes inhérentes à toute planification de gestion des ressources naturelles. Les plans doivent se focaliser sur les changements, les menaces et les réponses. Des technologies comme les outils de modélisation informatique sont là pour nous aider (Pressey et al., 2007).

Les actions de mitigation et d'adaptation aux changements sont sous-tendues par le besoin d'une meilleure compréhension des processus qui font que ces influences entraînent de tels changements dans l'environnement. Qu'est-ce qui renforce la résilience et la 


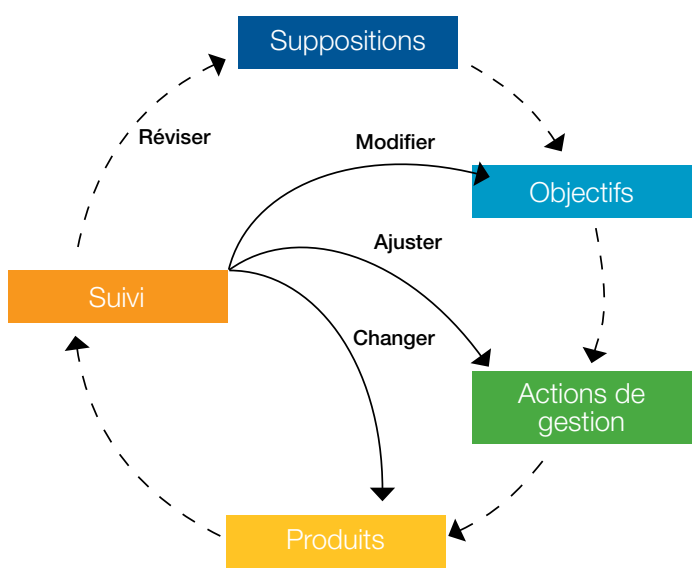

Figure 22.1 Cycles de rétro-informations associés à la gestion adaptative (CBD, 2003))

solidité écologiques et quels sont les points de basculement vers l'extinction ? De plus, même si l'on reconnaît que l'environnement a une valeur intrinsèque, le fait d'améliorer la compréhension des nombreuses valeurs économiques de la nature permettra d'étayer le dossier économique qui invitera à investir dans l'environnement. Maintenant que la communauté mondiale s'intéresse aux Objectifs du millénaire pour le développement (OMD) et, peut-être, à un cadre d'action post-2015, une meilleure compréhension du rôle de l'environnement, spécialement dans la réduction de la pauvreté et le développement, va certainement aider à garantir des investissements suffisants dans la conservation. L'Économie des écosystèmes et de l'environnement (TEEB), une analyse de la valeur de la nature qui sera lancée à la Conférence des Parties à la Convention sur la diversité biologique (CDB COP 10) en 2010, constituera une avancée majeure à ce point de vue. De plus, en appliquant les théories économiques à la gestion des écosystèmes, nous pouvons avoir de nouveaux points de vue sur la gestion des ressources et mieux comprendre les compensations qui sont faites lorsque l'on décide d'utiliser, ou pas, ces ressources (Perrings, 2006).

S'il est nécessaire de mieux comprendre les impacts et les options des différentes actions, la mise en œuvre efficace des décisions exige d'établir des partenariats entre secteurs et entre philosophies. Les entreprises, les programmes gouvernementaux, l'aide au développement et les communautés locales devront se rejoindre dans des objectifs communs. Les structures institutionnelles évoluent, les perceptions changent, et les nouvelles technologies nous ouvrent de nouvelles opportunités. Le développement d'une société toujours plus mondiale suscite des problèmes mais aussi des opportunités. Pour les affronter d'une façon qui assure la qualité de vie des générations présente et futures, il nous faudra de nouvelles visions et de nouvelles approches.

L'innovation est capitale dans le monde d'aujourd'hui, et c'est un ingrédient clé pour répondre et s'adapter aux changements qui résultent d'un monde toujours plus complexe, caractérisé par une population croissante et une base de ressources en déclin. L'innovation restera donc une obligation et aussi un espoir pour le monde durable dont beaucoup rêvent.

Comment la conservation peut-elle donner une impulsion à l'innovation ? Quelles sont les idées les plus novatrices qui émergent du CMN ? Qui sont les nouveaux partenaires ? Ce livre montre la voie vers quelques réponses.

Le pragmatisme pourrait être la clé de tout, le fait de se rendre compte que les solutions gagnantgagnant ne seront peut-être pas possibles et d'accepter des compensations qui permettront au moins des options «plus gagnant moins perdant».

\section{PROMOUVOIR UNE GOUVERNANCE EFFICACE}

La gouvernance, ce sont les interactions entre les structures, les processus et les traditions qui déterminent comment le pouvoir et les responsabilités sont exercés, comment les décisions sont prises et comment les citoyens ou les autres parties prenantes ont leur mot à 
dire dans la gestion des ressources naturelles. La gouvernance est le moyen d'atteindre un résultat et non le résultat lui-même. Elle se fait à de multiples niveaux (de local à mondial), dans des secteurs multiples (publics et privés) et dans de multiples cultures. Idéalement, toutes ces activités devraient se renforcer l'une l'autre, de sorte que les décisions prises au niveau international rendraient possibles les actions au niveau local. Si la gouvernance échoue, les conséquences peuvent être pires que la seule perte des ressources naturelles. Comme le disent Milledge et al. (2007), "les manques de gouvernance [dans le secteur forestier] risquent en fin de compte d'affecter les probabilités d'atteindre les objectifs nationaux de croissance économiques et de réduction de la pauvreté ».

Les définitions de ce qu'est la «bonne gouvernance » ont fait l'objet de nombreuses analyses (Bosselman et al., 2008). La Convention sur la diversité biologique a reconnu, dans sa Décision VII/11, que la bonne gouvernance est essentielle pour l'application de son approche par écosystèmes.

Dans plusieurs résolutions (CMN 3.012 et CMN 4.037) et dans son Programme, l'UICN a reconnu l'importance d'une gouvernance efficace et elle a défini comme suit les principes qui sous-tendent la bonne gouvernance :

- Transparence - ouverture dans les prises de décisions

- Accès à l'information et à la justice communication précise, effective et ouverte

- Participation de tous - véritable implication dans les prises de décisions

- Cohérence - une approche logique

- Subsidiarité - décisions prises au niveau approprié le plus bas

- Respect des droits de l'homme - imbriqués dans la «bonne » gouvernance environnementale

- Responsabilité - pour les performances économiques, sociales et environnementales
- Respect de la loi - application correcte, transparente et cohérente des dispositions légales à tous les niveaux.

La vision de l'UICN, « un monde juste qui valorise et préserve la nature » ne sera tout simplement pas réalisable si ces principes ne sont pas le fondement de notre travail de conservation. Non seulement c'est un impératif éthique, mais c'est aussi raisonnable : une conservation efficace ne peut se réaliser que lorsque ces principes fondamentaux sont intégrés dans notre travail.

\section{EQUITÉ, ET SPÉCIALEMENT ÉGALITÉ ENTRE LES SEXES}

L'UICN a pris un engagement envers l'équité dans sa vision et dans sa mission tout comme dans les nombreuses déclarations politiques, telles que sa Politique d'équité entre les sexes et les politiques en matière d'approches basées sur les droits. Le travail accompli au sein de l'UICN pour promouvoir l'égalité se fonde sur deux principes :

(1) l'égalité entre les sexes est une condition préalable de la conservation puisque les femmes représentent approximativement la moitié des utilisateurs des ressources naturelles et que, sans leur soutien, aucune politique de conservation ne pourrait être efficace ni/ou durable ;

(2) la conservation de la diversité biologique est une occasion de promouvoir l'égalité entre les sexes parce qu'elle favorise l'examen des pratiques existantes et l'introduction de nouvelles pratiques qui offrent la possibilité d'accroître le pouvoir des femmes.

Ce volume le reconnaît à plusieurs reprises, les femmes sont plus vulnérables face aux conditions changeantes (climat, catastrophes, pauvreté). D'autre part, l'UICN favorise une approche qui va bien au-delà de la simple considération du fait que les femmes forment un groupe marginalisé 
et qui souligne le rôle important que jouent les femmes dans la gestion des ressources naturelles. Elle reconnait aussi les femmes comme des sources de connaissances et de compétences essentielles pour la conservation.

Les recherches montrent que les projets de gestion environnementale qui incluent la participation des femmes (et donc leur expérience et leurs connaissances traditionnelles en gestion des ressources) sont plus efficaces (IRC, 1988). La Banque mondiale a analysé 121 projets ruraux de fourniture d'eau et a découvert que la participation des femmes comptait parmi les variables les plus fortement liées à l'efficacité des projets. Qui plus est, elle a trouvé que le fait de ne pas tenir compte des différences et des inégalités entre les sexes pouvait entraîner l'échec des projets (Narayan, 1995).

Tout au long de ce volume, nous avons vu que l'équité et l'égalité entre les sexes sont un sujet de préoccupation dans de nombreux domaines discutés, de la réduction de la pauvreté aux changements climatiques en passant par la gestion de l'eau et des énergies. Si l'on intègre des femmes comme actrices à part entière de la gouvernance et que l'on tient compte de leurs connaissances, il est possible d'augmenter significativement l'efficacité et la durabilité des initiatives de conservation. La question de l'équité et de son importance s'exprime souvent en termes de coûts si elle n'est pas prise en compte, en termes de bénéfices si elle l'est. Prenons le cas de l'égalité entre les sexes: dans aucune région les hommes et les femmes n'ont les mêmes droits sociaux, économiques et juridiques, et le résultat de cette inégalité est analysé dans un rapport de la Banque mondiale (2001). Il montre que les coûts de l'inégalité entre les sexes peuvent inclure une plus grande incidence du SIDA, la malnutrition, une plus grande fertilité et une plus forte mortalité infantile. Tout cela peut aussi avoir des impacts sur l'environnement.
Agarwal (2002) qui faisait un rapport sur la gestion communautaire de la forêt en Inde notait que plusieurs inégalités fondamentales (par exemple le partage inégal du travail entre hommes et femmes, un accès inégal aux ressources, des normes sociales et la perception du rôle des femmes) aboutissaient à une moindre participation des femmes à la gestion des ressources dont elles dépendaient et aussi à une diminution des bénéfices. En effet, ceux-ci sont souvent répartis sur la base des foyers et ce sont les hommes qui se voient accorder les bénéfices au nom de tout le ménage. Comme nous le notons plus bas, la participation pleine et entière est un facteur important pour des décisions efficaces en matière de gestion de la conservation et de l'utilisation durable des ressources.

\section{APPROCHES FONDÉES SUR LES DROITS}

Pour l'UICN, une approche de la conservation fondée sur les droits signifie une conservation qui intègre la considération et le respect des droits de l'homme, des droits fonciers et des droits d'accès aux ressources et/ou des droits coutumiers des populations autochtones et des communautés locales (UICN, 2008e). L'UICN a adopté cette politique en reconnaissant que certaines pratiques de conservation, comme les sédentarisations ou les déplacements forcés, pouvaient avoir des effets délétères sur le bien-être des personnes et, par son Centre du droit de l'environnement (CDE), elle a préparé un ensemble de principes concernant les droits de l'homme dans la conservation (Encadré 22.2).

Scherr (1999) note que le fait de reconnaître que les droits de propriété sur des ressources telles que des terres, de l'eau ou des arbres sont des atouts importants pour les foyers locaux s'est avéré avoir un rôle fondamental dans le lien entre pauvreté et environnement. Gbetibouo (2009), qui étudiait les agriculteurs d'Afrique australe, 
Encadré 22.2 Principes concernant les droits humains dans la conservation préparés par le Centre du droit de l'environnement

1. Promouvoir l'obligation pour tous les acteurs, Etats ou non, qui planifient ou qui sont déjà engagés dans des politiques, des projets, des programmes ou des activités qui ont des implications pour la nature, d'assurer à toutes les personnes et les populations étatiques et non étatiques les droits essentiels et les procédures qui sont garanties par le droit national et international.

\section{Prévoir une évaluation préalable de la portée} des politiques, projets, programmes ou activités de conservation, de façon à ce que tous les liens entre les droits de l'homme et l'environnement soient identifiés, et que toutes les personnes qui pourraient être touchées soient informées et consultées.

3. S'assurer que la planification et la mise en œuvre des politiques et actions de conservation tiennent compte de cette évaluation préalable, soient fondées sur des décisions raisonnées et ne portent donc pas préjudice aux personnes vulnérables, mais soutiennent dans la mesure du possible la réalisation de leurs droits dans le contexte de l'utilisation de la nature et des ressources naturelles.

4. Intégrer des orientations et des outils dans la planification des projets et des programmes pour assurer le suivi et l'évaluation de toutes les interventions et vérifier leurs conséquences pour les droits fondamentaux des personnes concernées ou potentiellement touchées, ce qui garantira une plus grande transparence et servira de point de départ à un processus de rétro-information.

5. Soutenir le renforcement des systèmes de gouvernance sur des questions relatives aux cadres juridiques, politiques, institutionnels et aux procédures susceptibles de garantir les droits des populations locales dans le contexte de la conservation et de l'utilisation durable des ressources.

Source : CMN 4.056 facteurs qui affectaient la capacité de s'adapter aux changements climatiques. Fisher et Oviedo (2008) notent que « les droits environnementaux peuvent parfois être interprétés d'une manière qui sape les droits de l'homme » et demandent que les discussions au sujet des approches de la conservation basées sur les droits aillent audelà des simples droits de propriété/d'accès aux ressources pour englober un plus large spectre de questions, comme les droits humains et la justice.

Le fait de transmettre l'autorité aux populations locales s'est avéré fructueux dans la conservation des forêts en Tanzanie (Barrow et al., 2003), en Ethiopie (IIRR, 2000) et en Chine (Oviedo, 2006). Les approches fondées sur les droits se sont révélées vitales pour soutenir le mode de vie et aussi la culture des populations autochtones. Citons comme exemples le fait d'accorder des titres et des droits sur les forêts du sud du Guyana aux Wai Wai, ce qui leur a permis de créer la première aire protégée du Guyana, qui est aussi la seule aire amérindienne (Janki et Sose, 2008), ou encore la cogestion de Waza Logone, au Cameroun. Au début des années 1990, l’UICN a initié le développement d'organisations de cogestion qui passe notamment par la reconnaissance et l'extension du droit des communautés locales d'utiliser des terres à l'intérieur des parcs. Le fait d'avoir confié l'autorité de la gestion aux communautés dans la «zone périphérique du parc a abouti à un meilleur état de l'écosystème, et les membres des 
communautés participantes ont noté des résultats positifs tant en termes d'accès aux ressources que de réduction des conflits »(Scholte et al., 2006).

\section{PARTICIPATION, TRANSPARENCE ET RESPONSABILITÉ}

Le Principe de Rio 10 déclare que « la meilleure façon de traiter les questions d'environnement est d'assurer la participation de tous les citoyens concernés, au niveau qui convient ". Il a été démontré que la conservation participative, comme partie de la gestion d'une ressource naturelle, aboutit à un meilleur état de cette ressource - ex. les forêts de Tanzanie (Bloomley et al., 2008).

Les approches inclusives favorisent aussi l'intégration de nombreux éléments isolés mais pertinents dans la gestion durable des ressources. Par exemple, le Gouvernement de Terre-Neuve et du Labrador a voulu consolider la gestion de la sous-espèce d'ours polaire qui vit dans le Détroit de Davis et a créé un document inclusif qui combine les connaissances locales, autochtones et scientifiques dans un Plan de gestion qui est un " document vivant " qui continuera à être ajusté en fonction des nouvelles informations disponibles. Le Plan de gestion de Terre-Neuve et du Labrador va plus loin que la plupart des plans de gestion d'espèces parce qu'il reprend non seulement les connaissances scientifiques et le savoir local, mais aussi les connaissances traditionnelles des anciens Nain au sujet de l'habitat des ours polaires, des changements climatiques, des rencontres avec les hommes et de la chasse traditionnelle

(MacLeod, 2008).

\section{LE DERNIER MOT}

Construire un avenir durable exigera les efforts concertés de toute la société. Dans Le passage à la durabilité : vers un monde humain et divers, Adams et Jeanrenaud (2008) soulignent le besoin d'" une économie pour une planète unique ", $\mathrm{d}$ '« un mouvement environnemental mondial renouvelé " et d'" une architecture institutionnelle qui œuvre pour le changement». Le fait de ne pas agir maintenant coûterait très cher, pas seulement au point de vue financier, mais aussi pour notre avenir. La préservation des gènes, des espèces et des écosystèmes va permettre de grandes économies à long terme, mais elle exigera d'importants investissements aujourd'hui. Que faudra-t-il faire pour convaincre les hommes de faire ces investissements?

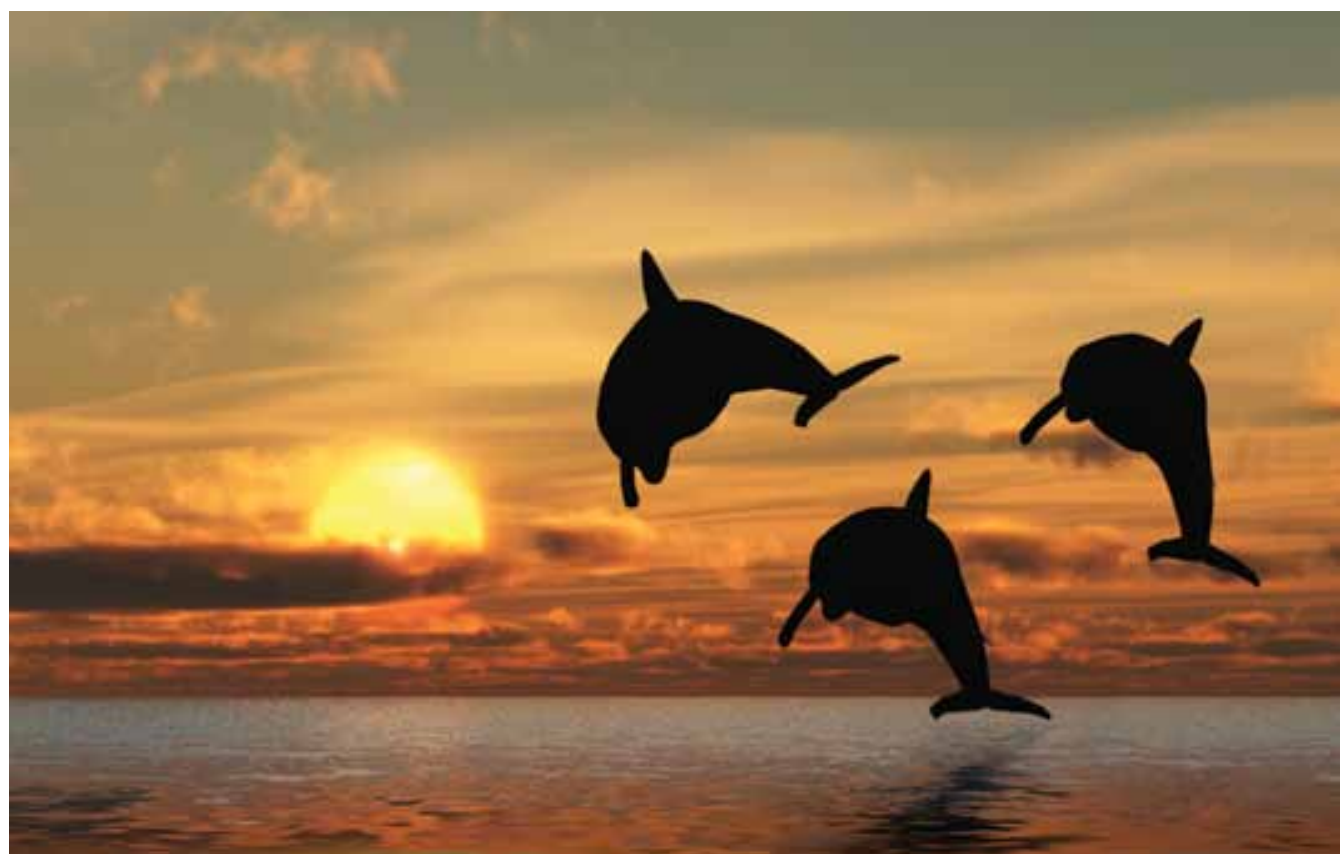




\section{Bibliographie}

Adams, W.M. and S.J. Jeanrenaud. 2008. Le passage à la durabilité : vers un monde humain et divers. IUCN, Gland, Switzerland.

Agarwal, B. 2002. Gender Inequality, Cooperation and Environmental Sustainability. http:// www.santafe.edu/research/publications/ workingpapers/02-10-058.pdf

Aguilar, G. and A. Iza. 2006. Gobernanza de aguas compartidas: aspectos juridicos e institucionales. UICN Mesoamerica, San Jose, Costa Rica. http://data.iucn.org/dbtw-wpd/edocs/EPLP058.pdf

Allison, E.H., A.L. Perry, M. Badjeck, W.N. Adger, K. Brown, D. Conway, A.S. Halls, G.M. Pilling, J.D. Reynolds, N.L. Andrew and N.K. Dulvy. 2009. Vulnerability of national economies to the impacts of climate change on fisheries. Fish and Fisheries 4 Feb 2009. doi: 10.1111/j.1467-2979.2008.00310.x

Altieri, M. 1995. Agroecology: The Science of Sustainable Agriculture. Westview Press, Boulder, CO, USA.

Amend, S. and T. Amend (eds.). 1995. National Parks without People? The South American Experience. IUCN, Gland, Switzerland.

Anon. 1994. The Earth Charter. www.earthcharter.org

Asahi Glass Foundation. 2008. Summary of the 17th Questionnaire on Environmental Problems and the Survival of Humankind. http://www.af-info.or.jp/ en/questionnaire/doc/2008jresult_fulltext.pdf
Asian Development Bank (ADB). 2005. Assessment of tsunami recovery implementation in Hambantota district. http://www.adb.org/ Documents/Reports/Rebuilding-Sri-Lanka/ Hambantota-team.pdf

AT Kearney. 2009. Green Winners. http://www. atkearney.com/images/global/pdf/Green_ winners.pdf

Badman, T., B. Bomhard, A. Fincke, J. Langley, P. Rosabal and D. Sheppard. 2009. World Heritage in Danger. IUCN, Gland, Switzerland.

Baillie, J., C. Hilton-Taylor and S.N. Stuart. 2004. 2004 IUCN Red List of Threatened Species: a global species assessment. IUCN, Gland, Switzerland. 191 pp.

Baker, C.S., J.C. Cooke, S. Lavery, M.L. Dalebout, Y.U. Ma, N. Funahashi, C. Carraher and R.L. Brownell Jr. 2007. Estimating the number of whales entering trade using DNA profiling and capture-recapture analysis of market products. Molecular Ecology. doi: 10.1111/j.1365-294X.2007.03317.x

Balian, E.V., H. Segers, C. Lévêque and K. Martens. 2008. The freshwater animal diversity assessment: an overview of the results. Hydrobiologia 595: 627-637.

Balmford, A., A. Bruner, P. Cooper, R. Costanza, S. Farber, R.E. Green, M. Jenkins, P. Jefferiss, V. Jessamy, J. Madden, K. Munro, N. Myers, S. Naeem, J. Paavola, M. Rayment, S. Rosendo, J. Roughgarden, K. Trumper and 
R.K. Turner. 2002. Economic Reasons for Conserving Wild Nature. Science 297: 950-953.

Barrow, E. and W. Mlenge. 2003. Trees as Key to Pastoralist Risk Management in Semi-Arid Landscapes in Shinyanga, Tanzania, and Turkana, Kenya. International Conference on Rural Livelihoods, Forest and Biodiversity. CIFOR, Bonn, Germany.

Baskin, Y. 2002. A Plague of Rats and Rubber Vines: the growing threat of species invasions. Island Press, Washington DC, USA. 377 pp.

Benstead, J.P., P.H. De Rham, J.-L. Gattoliat, F.-M. Gibon, P.V. Loiselle, M. Sartori, J.S. Sparks and M.L.J. Stiassny. 2003. Conserving Madagascar's freshwater biodiversity. Bioscience 53(11): 1101-1111.

Benton-Short, L. and J. Rennie-Short. 2007. Cities and Nature. Routledge, London, UK.

Benyus, J. 1997. Biomimicry: Innovation inspired by nature. Perennial, New York, USA.

Bergstrom, D., A. Lucieer, K. Kiefer, J. Wasley, L. Belbin, T.K. Pedersen and S.L. Chown. 2009. Indirect effects of invasive species removal devastate World Heritage Island. Journal of Applied Ecology 46: 73-81.

Bishop, J., S. Kapila, F. Hicks, P. Mitchell and F. Vorhies. 2008. Building Biodiversity Business. Shell International Limited, London, UK and IUCN, Gland, Switzerland. 164 pp. http://data.iucn.org/dbtw-wpd/ edocs/2008-002.pdf

Bishop, J., S. Kapila, F. Hicks, P. Mitchell and F. Vorhies. 2009. New Business Models for Biodiversity Conservation. Journal of Sustainable Forestry 28: 285-303. doi: 10.1080/10549810902791481

Bishop, K., N. Dudley, A. Phillips and S. Stolton. 2004. Speaking a common language. Cardiff University, Cardiff, UK, IUCN, Gland,
Switzerland and UNEP-WCMC, Nairobi, Kenya. 191 pp. http://data.iucn.org/dbtwwpd/edocs/2004-049.pdf

Blann, K. 2006. Habitat in Agricultural Landscapes: How Much is Enough? A State-of-the-Science Literature Review. Defenders of Wildlife, West Linn, OR, USA.

Blomley, T., K. Pfliegner, J. Isango, E. Zahabu, A. Ahrends and N. Burgess. 2008. Seeing the wood for the trees: an assessment of the impact of participatory forest management on forest condition in Tanzania. Oryx 42: 380-391.

Bojo, J., K. Green, S. Kishore, S., Pilapitiva and R.C. Reddy. 2004. Environment in Poverty Reduction Strategies and Poverty Reduction Support Credits. World Bank Environment Department Paper No. 102.

Bonkoungou, E.G. and M. Naimir-Fuller. 2001. Biodiversity in Drylands: challenges and opportunities for conservation and sustainable use. The Global Drylands Partnership, UNDP, New York, USA.

Borrini-Feyerabend, G., A. Kothari and G. Oviedo. 2004. Indigenous and Local Communities and Protected Areas: Towards Equity and Enhanced Conservation. IUCN, Gland, Switzerland and Cambridge, UK.

Bosselmann, K., R. Engel and P. Taylor. 2008. Governance for Sustainability - Issues, Challenges, Successes. IUCN, Gland, Switzerland. xvi +260 pp.

Boucher, J. and W.F. Doolittle. 2002. Something new under the sea. Nature 417: 27-28.

Bowen, G., J. Leonard, I. Wassenaar and K.A. Hobson. 2005. Global application of stable hydrogen and oxygen isotopes to wildlife forensics. Oecologia 143: 337-348. doi: 10.1007/s00442-004-1813-y 
Braby C.E. and G.N. Somero. 2006a. Ecological gradients and relative abundance of native (Mytilus trossulus) and invasive (Mytilus galloprovincialis) blue mussels in the California hybrid zone. Marine Biology 148: 1249-1262.

Brashares, J.C., P. Arcese, M.K. Sam, P.B. Coppolillo, A.R.E. Sinclair and A. Balmford. 2004. Bushmeat Hunting, Wildlife Declines, and Fish Supply in West Africa. Science 306: 1180-1183.

Bräuer, I., R. Müssner, K. Marsden, F. Oosterhuis, M. Rayment, C. Miller et al. 2006. The use of market incentives to preserve biodiversity. (Final Report - A project under the Framework Contract for Economic Analysis ENV.G.1/ FRA/2004/0081.)

Bray, M. 2006. Reviewe of Computer Energy Consumption and Potential Savings. White Paper. Sponsored by Dragon Systems Software Limited (DssW). http://www. dssw.co.uk/research/computer_energy_ consumption.pdf

Brown, L. 2004. Outgrowing the Earth: The Food Security Challenge in an Age of Falling Water Tables and Rising Temperatures. W.W. Norton, New York, USA.

Bruner, A.G., R.E. Gullison and A. Balmford. 2004. Financial Costs and Shortfalls of Managing and Expanding Protected-Area Systems in Developing Countries. Bioscience 54: 1119-1126.

Burns, C.E., K.M. Johnston and O.J. Smitz. 2003. Global climate change and mammalian species diversity in U.S. national parks. Proceedings of the National Academy of Sciences 100: 11474-11477.

Burrowes, P.A., R.L. Joglar and D.E. Green. 2004. Potential causes for amphibian declines in Puerto Rico. Herpetologica 60: 141.
Business and Biodiversity Offsets Programme (BBOP). 2009. Business, Biodiversity Offsets and BBOP: An Overview. BBOP, Washington DC, USA. http://www.forest-trends.org/ biodiversityoffsetprogram/guidelines/ overview.pdf

Butchart, S. 2008. Red List Indices to measure the sustainability of species use and impacts of invasive alien species. Bird Conservation International 18: S245-S262. doi: 10.1017/ S095927090800035X

Butchart, S.H.M., A.J. Stattersfield, J. Baillie, L.A. Bennun, S.N. Stuart, H.R. Akcakaya, C. Hilton-Taylor and G.M. Mace. 2005. Using Red List Indices to measure progress towards the 2010 target and beyond. Phil. Trans. R. Soc. B 360 : 255-268.

Carbon Footprint. http://www.carbonfootprint. com/energyconsumption.html

Carpenter, S.R., P. Pingali, E. Bennett and M. Zurek (eds.). 2005. Ecosystems and Human Well-being: Scenarios. Island Press, Washington DC, USA.

Cassman, K.G. and S. Wood. 2005. Cultivated Systems. Ecosystems and Human Well-being: Current State and Trends, Volume 1. 745-794. In Hassan, R., R. Scholes and N. Ash (eds.). Island Press, Washington DC, USA.

Cello, J., A.V. Paul and E. Wimmer. 2002. Chemical synthesis of polio virus cDNA: Generation of infectious virus in the absence of natural template. Science 297: 1016-1018.

Chan, K.M., M.R. Shaw, D.R. Cameron, E.C. Underwood and G.C. Daily. 2006. Conservation Planning for Ecosystem Services. PLoS Biology 4: e379.

Chivian, E. and A. Bernstein (eds). 2008. Sustaining Life: How Human Health Depends on Biodiversity. Oxford University Press, New York, USA. 
Clay, J. 2004. World Agriculture and the Environment: A Commodity-By-Commodity Guide to Impacts and Practices. Island Press, Washington DC, USA.

Clergeau, P., S. Croci, J. Jokimäki, M.-L.

Kaisanlahti-Jokimäki and M. Dinetti. 2006. Avifauna homogenization by urbanization: Analysis at different European latitudes. Biological Conservation 127: 336-244.

Coad, L., A. Campbell, L. Miles, K. Humphries. 2008. The Costs and Benefits of Protected Areas for Local Livelihoods: a revieze of the current literature. Working Paper. UNEP World Conservation Monitoring Centre, Cambridge, UK. http:// www.unep-wcmc.org/climate/pdf/Coad_et_ al_2008_Working_Paper.pdf

Cohen, J.E. 2005. Human Population Grows Up. Scientific American Sept 2005: 48-55.

Collier, P. 2003. Breaking the Conflict Trap: Civil war and development policy. Oxford University Press, London, UK.

Comprehensive Assessment of Water Management in Agriculture. 2007. Water for Food, Water for Life: A Comprehensive Assessment of Water Management in Agriculture. Earthscan, London, UK, International Water Management Institute, Colombo, Sri Lanka.

Convention on Biological Diversity (CBD). 2003. Report of the Fourth open-ended WORKSHOP ON Sustainable Use of Biological Diversity UNEP/CBD/WS Sustainable Use 4/4. http:// www.cbd.int/doc/meetings/suse/wssuse-04/ official/wssuse-04-04-en.doc

Convention on Biological Diversity (CBD). 2004a. Guidelines on biodiversity and tourism development. http://www.biodiv.org/doc/ publications/tou-gdl-en.pdf

Convention on Biological Diversity (CBD). 2004b. Programme of Work on Protected Areas. http://www.cbd.int/protected/pow.shtml
Convention on Biological Diversity Secretariat (CBD). 2006. Global Biodiversity Outlook 2. Montreal, Canada. $81+$ vii pp. http://www. cbd.int/gbo2/

Convention on Biological Diversity (CBD). 2008. Report of the first meeting of the second ad hoc technical expert group on biodiversity and climate change. UNEP/CBD/AHTEG/BD-CC-2/2/5. http://www.cbd.int/doc/meetings/cc/ahtegbdcc-02-02/official/ahteg-bdcc-02-02-05-en.doc

Convention on Biological Diversity (CBD). Principles of the Ecosystem Approach. http:// www.cbd.int/ecosystem/principles.shtml

Convention on Trade in Endangered Species (CITES). 2001. Multi-lateral agreements for conservation of hawessbill turtles. International Instruments Relevant to the Conservation of Hawksbill Turtles [And Their Habitats]. Background document prepared by IUCN/ ELC for CITES hawksbill turtle dialogue meetings held in 2001 and 2002. http:// www.cites.org/eng/prog/HBT/bg/multi_ agreement1.shtml

Costello, C., S.D. Gaines and J. Lynham. 2008. Can catch shares prevent fisheries collapse? Science 321: 1678-1681.

Crawford, J.A. and R.V. Fiorentino. 2005. The Changing Landscape of Regional Trade Agreements. WTO Discussion paper No. 8 . http://www.wto.int/english/res_e/booksp_e/ discussion_papers8_e.pdf

Cumberlidge, N. et al. 2009. Freshwater crabs and the biodiversity crisis: Importance, threats, status, and conservation challenges. Biol. Conserv. doi: 10.1016/j.biocon.2009.02.038. In press.

Daily, G.C. (ed.). 1997. Nature's Services: Societal Dependence on Natural Ecosystems. Island Press, Washington DC, USA. 
Dalton, R. 2003. Mock turtles. Nature. 219-220.

Darnall, N. and S.R. Sides. 2008. Assessing the Performance of Voluntary Environmental Programs: Does Certification Matter? Policy Studies Journal 36(1). http://ssrn.com/ abstract $=1030622$

Darwall, W., K. Smith, D. Allen, M. Seddon, G. McGregor Reid, V. Clausnitzer and V. Kalkman. 2008. Freshwater biodiversity - a hidden resource under threat. The 2008 Review of the IUCN Red List of Threatened Species. In Vié, J.C., C. Hilton-Taylor and S.N. Stuart (eds.). IUCN, Gland, Switzerland.

Darwall, W.R.T., K.G. Smith and D. Tweddle (eds.). 2008. The Status and Distribution of Freshwater Biodiversity in Southern Africa. IUCN, Gland, Switzerland.

Deffeyes, K.S. 2005. Beyond Oil: The View from Hubbard's Peak. Hill and Wang, New York, USA.

Derocher, A.E., N.J. Lunn and I. Stirling. 2004. Polar Bears in a warming climate. Integrative and Comparative Biology 44(2): 163-176.

DFID, EC, UNDP and World Bank. 2002. Linking Poverty Reduction and Environmental Management: Policy Challenges and Opportunities. DFID, London, UK.

\section{Distefano, E. 2005. Human-Wildlife Conflict} worldwide: collection of case studies, analysis of management strategies and good practices. http:// www.fao.org/sard/common/ecg/1357_en_ HWC_final.pdf

Dolman, S.J., M.P. Simmonds and S. Keith. 2002. Marine wind farms and cetaceans. International Whaling Commission IWC/SC/55/E4.

Dudley, N. (ed.). 2008. Lignes directrices pour l'application des catégories de gestion aux aires protégées. IUCN, Gland, Switzerland. $x+96$ pp. http://data.iucn. org/dbtw-wpd/edocs/PAPS-016-Fr.pdf
Dudley, N. and S. Stolton. 2005. The Role of forest protected areas in supplying water to the world's biggest cities. The Urban Imperative. 27-33. In Tryzna, T. (ed.). California Institute of Public Affairs, Sacramento, CA, USA.

Dukes, J.S. and H.A. Mooney. 2004. Disruption of ecosystem processes in western North America by invasive species. Revista Chilena de Historia Natural 77: 411-437.

Dyson, M., G. Bergkamp and J. Scanlon (eds.). 2003. Débit : les débits environnementaux, ce qu'il faut savoir. IUCN, Gland, Switzerland and Cambridge, UK. xiv +118 pp.

Earthtrack. 2008. Subsidies are an expensive way to remove greenhouse gases from the economy. http://www.earthtrack.net/earthtrack/library/ Carbon $\% 20$ efficiency $\% 20$ of $\% 20$ Subsidies.pdf

Ehrenfeld, J.G. 2003. Effects of exotic plant invasions on soil nutrient cycling processes. Ecosystems 6(6): 503-523.

Ehrlich, P. and A. Ehrlich. 2008. The Dominant Animal. Island Press, Washington DC, USA.

Eken, G., L. Bennun, T.M. Brooks, W. Darwall, L.D.C. Fishpool, M. Foster, D. Knox, P. Langhammer, P. Matiku, E. Radford, P. Salaman, W. Sechrest, M.L. Smith, S. Spector and A. Tordoff. 2004. Key biodiversity areas as site conservation targets. BioScience 54: 1110-1118.

Eliasch, J. 2008. The Eliasch Reviere: Climate Change: Financing Global Forests. United Kingdom Office of Climate Change (OCC), London, UK. http://www.occ.gov.uk/activities/eliasch.htm.

Ellis W.N., J.H. Donner and J.H. Kuchlein. 1997. Recent shifts in phenology of Microlepidoptera, related to climatic change (Lepidoptera). Entomologische Berichten 57: 66-72.

Elsayed, S. 2009. Energy Access for Development. EarthTrends Update May 2009. 
Emerton, L., J. Bishop and L. Thomas. 2006. Sustainable Financing of Protected Areas: A global reviewe of challenges and options. IUCN, Gland, Switzerland and Cambridge, UK. $x+97$ pp. http://app.iucn. org/dbtw-wpd/edocs/PAG-013.pdf

Engelhard, G.H. and M. Heino. 2006. Climate Change and Condition of Herring (Clupea Harengus) Explain Long-Term Trends in Extent of Skipped Reproduction. Interim report 06-008. International Institute for Applied Systems Analysis, Laxenburg, Austria.

Engler, M. 2008. The Value of International wildlife Trade. TRAFFIC Bulletin 22(1): 4-5.

Ervin, D.E., R. Welsh, S.S. Batie and C. Line Carpentier. 2003. Towards an ecological systems approach in public research for environmental regulation of transgenic crops. Agriculture, Ecosystems and Environment 99: 1-14. doi: 10.1016/S0167-8809(03)00145-2

European Commission (EC) and German Ministry for the Environment, BMU. 2008. TEEB The Economics of Ecosystems and Biodiversity: An interim report. European Communities, Brussels, Belgium. http://ec.europa.eu/ environment/nature/biodiversity/economics/ pdf/teeb_report.pdf

European Commissions (EC). 2008. Commission Recommendation of 07/02/2008 on a code of conduct for responsible nanosciences and nanotechnologies research. http://ec.europa.eu/nanotechnology/ pdf/nanocode-rec_pe0894c_en.pdf

European Environment Agency (EEA). 2005. The European Environment: State and Outlook 2005. http://www.eea.europa.eu/publications/ state_of_environment_report_2005_1

Farber, S., R. Costanza, D.L. Childers, J. Erickson, K. Gross, M. Grove, C.S. Hopkinson, J. Kahn, S. Pincetl, A. Troy, P. Warren and M. Wilson. 2006. Linking Ecology and Economics for Ecosystem Management. BioScience 56(2): 121-133.
Fernández-Juricic, E. and J. Jokimäki. 2001. A habitat island approach to conserving birds in urban landscapes: case studies from southern and northern Europe. Biodiversity and Conservation 10: 2023-2043.

Fisher, R. and G. Oviedo. 2008. Rights-based approaches to forest conservation. Arborvitae 36. http://cmsdata.iucn.org/downloads/ av_36_english.pdf

Fisher, R.J., S. Maginnis, W.J. Jackson, E. Barrow and S. Jeanrenaud. 2005. Poverty and Conservation: Landscapes, People and Power. IUCN, Gland, Switzerland and Cambridge, UK. xvi +148 pp.

Fisher, R., S. Maginnis, W. Jackson, E. Barrow and S. Jeanrenaud. 2008. Linking Conservation and Poverty Reduction. Earthscan, London, UK. 146 pp.

Food and Agriculture Organization (FAO). 2001a. Conservation Agriculture: Case Studies in Latin America and Africa. FAO, Rome, Italy.

Food and Agriculture Organization (FAO). (2001b). Les femmes, utilisatrices, conservatrices et gestionnaires de la diversité biologique. SD dimensions. http://www.fao.org/sd/2001/ PE1201_fr.htm

Food and Agriculture Organization (FAO). 2005. Évaluation des Ressources Forestières Mondiales. http://www.fao.org/docrep/009/a0400f/ a0400f00.htm

Food and Agriculture Organization (FAO). 2008a. La situation mondiale des pêches et de l'aquaculture. ftp://ftp.fao.org/docrep/fao/011/ i0250f/i0250f.pdf

Food and Agriculture Organization (FAO). 2008b. Quelques problèmes auxquels sont confrontés les pêcheurs et les aquaculteurs. 2ème partie. $\mathrm{ftp}: / / \mathrm{ftp}$. fao.org/docrep/fao/011/i0250f/i0250f02.pdf

Food and Agriculture Organization (FAO). 2008c. La situation mondiale de l'alimentation et de 
l'agriculture 2008. ftp://ftp.fao.org/docrep/ fao/011/i0100f/i0100f.pdf

Food and Agriculture Organization (FAO). 2008d.

L'état de l'insécurité alimentaire dans le monde 2008. http://www.fao.org/docrep/011/i0291e/ i0291e00.htm

Food and Agriculture Organization (FAO). 2009a. Situation des forêts du monde 2009. ftp://ftp.fao. org/docrep/fao/011/i0350f/i0350f.pdf

Food and Agriculture Organization (FAO). 2009b.

Partenariat de collaboration sur les forêts. http:// www.fao.org/forestry/44935/fr/

Food and Agriculture Organisation (FAO). AQUASTAT database. http://www.fao.org/ $\mathrm{nr} /$ water/aquastat/data/query/index.html

Ford, J. 2007. Emerging trends in climate change policy: the role of adaptation. Journal of Climate 3: 2. 12 pp. http://www.ucl.ac.uk/ ippr/download/volume-3-2/Ford.pdf

Fudge, S. and G.A. Rose. 2008. Life history covariation in a fishery depleted Atlantic cod stock. Fisheries Research 92(1): 107-113.

Fuller, R.A. and K.J. Gaston. 2009. The scaling of green space coverage in European cities. Biological Letters. doi: 10.1098/ RSBI.2009.0010.

Funk, C., M.D. Dettinger, J.C. Michaelsen, J.P. Verdin, M.E. Brown, M. Barlow and A. Hoell. 2008. Warming of the Indian Ocean threatens eastern and southern African food security but could be mitigated by agricultural development. Proceedings of the National Academy of Sciences 105: 11081-11086.

Gadgil, M. and F. Berkes. 1991. Traditional Resource Management Systems. Resource Management and Optimization 8(3-4): 127-141.

Gardner, G. 2002. Invoking the Spirit: Religion and Spirituality in the Quest for a Sustainable World. Worldwatch Institute, Washington DC, USA.
Gazeau, F., C. Quiblier, J.M. Jansen, J.-P. Gattuso, J.J. Middelburg and C.H.R. Heip. 2007. Impact of elevated CO2 on shellfish calcification. Geophysical Research Letters 34: L07603. doi: 10.1029/2006GL028554

Gbetibouo, G.A. 2009. Understanding Farmers' Perceptions and Adaptations to Climate Change and Variability: The Case of the Limpopo Basin, South Africa. IFPRI Discussion Paper 00849. http://www.ifpri.org/pubs/dp/ IFPRIDP00849.pdf

Gibson, C., S. Valenti, S. Fowler and S. Fordham. 2008. The Conservation Status of Northeast Atlantic Chondrichthyans: Report of the IUCN Shark Specialist Group Northeast Atlantic Regional Red List Workshop. http://cmsdata. iucn.org/downloads/shark_report_1.pdf

Gilman, E., D. Kobayashi and M. Chaloupka. 2008. Reducing seabird bycatch in the Hawaii longlinetuna fishery. Endangered Species Research. http://cmsdata.iucn.org/downloads/ reducing_seabird_bycatch.pdf

Girardet, H. 1999. Greening Urban Society. Imagine Tomorrow's World. 110-120. In McNeely, J.A. (ed.). IUCN, Gland, Switzerland.

Global Environment Facility (GEF). 2009. Biodiversity. http://www.gefweb.org/interior_ right.aspx?id=224

Global Footprint Network. 2008. http://www. footprintnetwork.org/en/index.php/GFN/ page/world_footprint/

Global Sustainable Tourism Criteria Partnership. 2008. Global sustainable tourism criteria. http:// www.sustainabletourismcriteria.org/index. php?option $=$ com_content\&task $=$ view $\&$ id $=58$ \&Itemid $=188$

Global Transboundary Protected Area Network (GTPAN). 2009. UNEP-WCMC Transboundary Protected Area Network Inventory - 2007. http:// 
www.tbpa.net/tpa_inventory.html

Global Water Partnership (GWP). 2000. Integrated

Water Resource Management. Global Water

Partnership Technical Advisory Committee

Paper 4. http://www.cepis.ops-oms.org/

bvsarg/i/fulltext/tac4/tac4.pdf

Global Water Partnership (GWP). 2009. Toolbox

- Integrated Water Resources Management.

http://www.gwptoolbox.org/index.

php?option=com_content $\&$ view $=$ article $\&$ id $=$ $8 \&$ Itemid $=3$

Goldin, O. 1997. The Ecology of the Critias and Platonic Metaphysics. The Greeks and the Environment. 73-82. In Westra, L. and T.M. Robinson (eds.). Rowman \& Littlefield Publishers, Lanham, MD, USA.

Goode, D. 2005. Connecting with Nature in a Capital City: The London Biodiversity Strategy. The Urban imperative. 75-85. In Tryzna, T. (ed.). California Institute of Public Affairs, Sacramento, CA, USA.

Green Indian States Trust (GIST). 2003-2008. Green Accounting for India's States and Union Territories Project Reports. GIST, Chennai, India.

Grimm, N.B., S.H. Faeth, N.E. Golubiewski, C.L. Redman, J. Wu, X. Bai and J.M. Briggs. 2008. Global change and the ecology of cities. Science 319: 756-760.

Hamann, A. and T. Wang. 2006. Potential Effects of Climate Change on Ecosystem and Tree Species Distribution. British Columbia Ecology 87(11): 2773-2786.

Hamilton, K., M. Sjardin, A. Shapiro and T. Marcello. 2009. Fortifying the Foundation: State of the Voluntary Carbon Markets 2009. Ecosystem Marketplace, New Carbon Finance. http://ecosystemmarketplace. com/documents/cms_documents/StateOf TheVoluntaryCarbonMarkets_2009.pdf
Hansen, J., M. Sato, R. Ruedy, K. Lo, D.W. Lea and M. Medina-Elizade. 2006. Global temperature change. Proceedings of the National Academy of Sciences 103: 14288-14293. doi: 10.1073/pnas.0606291103

Hansen, S. 2007. The Economic Case for investing in the Environment: A Review of Policies, Practice and Impacts of Relevance to Norwegian Partner Countries. Norad Report 6a/2007 Discussion. http://norad.no/en/Tools+and+publications/ Publications/Publication+Page?key=109662

Hanson, T., T.M. Brooks, G.A.B. da Fonseca, M. Hoffmann, J.F. Lamoreux, G. Machlis, C.G. Mittermeier, R.A. Mittermeier and J.D. Pilgrim. 2009. Warfare in Biodiversity Hotspots. Conservation Biology 23: 578-587.

Harvey C., F. Alpizar, M. Chacon and R. Madrigal. 2004. Assessing Linkages between Agriculture and Biodiversity in Central America: Historical Overview and Future Perspectives. The Nature Conservancy, San Jose, Costa Rica.

Hausman, R., L. Tyson and S. Zahidi. 2007. The Global Gender Gap Report 2007. World Economic Forum. Ref 112007. http://www. weforum.org/pdf/gendergap/report2007.pdf

Hockings, M., S. Stolton, F. Leverington, N. Dudley and J. Courrau. 2006. Évaluation de l'efficacité: Un cadre pour l'évaluation de l'efficacité de la gestion des aires protégées, 2ème édition. IUCN, Gland, Switzerland and Cambridge, UK. xiv +105 pp.

Howard, G. and S. Ziller. 2008. Alien alert plants for biofuel may be invasive. Bioenergy Business. July/August 2008: 14-16.

Howarth, R.W. and S. Bringezu (eds.). 2009. Biofuels: Environmental Consequences and Interactions with Changing Land Use. Proceedings of the Scientific Committee on Problems of the Environment (SCOPE) International Biofuels Project Rapid Assessment, 22-25 
September 2008, Gummersbach, Germany. http://cip.cornell.edu/biofuels/

Huntley, B., R.E. Green, Y.C. Collingham and S.G. Willis. 2007. A Climatic Atlas of European Breeding Birds. Lynx Edicions, Barcelona, Spain.

Hutton, J.M. and N. Leader-Williams. 2003.

Sustainable use and incentive-driven conservation: realigning human and conservation interests. Oryx 37: 215-226.

IIED. 2009. Environmental Mainstreaming. http:// www.environmental-mainstreaming.org Intergovernmental Panel on Climate Change (IPCC). 2007a. IPCC 4th Assessment ReportSummary for Policy Makers. http://www.ipcc.ch/ pdf/assessment-report/ar4/syr/ar4_syr_spm.pdf

Intergovernmental Panel on Climate Change (IPCC). 2007b. Summary for Policymakers. Climate Change 2007: Impacts, Adaptation and Vulnerability. Contribution of Working Group II to the Fourth Assessment Report of the Intergovernmental Panel on Climate Change. 7-22. In Parry, M.L., O.F. Canziani, J.P. Palutikof, P.J. van der Linden and C.E. Hanson (eds.). Cambridge University Press, Cambridge, UK. http://www.ipcc.ch/pdf/ assessment-report/ar4/wg2/ar4-wg2-spm.pdf

International Council on Mining and Minerals (ICMM) 2006. Good Practice Guidance for Mining and Biodiversity. http://www.icmm. com/page/1182/good-practice-guidance-formining-and-biodiversity

International Federation of Organic Agriculture Movements (IFOAM). 2000. Organic Agriculture and Biodiversity. IFOAM, Bonn, Germany.

International Institute of Rural Reconstruction (IIRR). 2000. Sustainable Agriculture Extension Manual. IIRR, Cavite, Philippines.

International Risk Governance Council (IRGC). 2007. Nanotechnology Risk Governance. IRGC
White Paper. http://www.irgc.org/IMG/pdf/ PB_nanoFINAL2_2_.pdf

International Risk Governance Council (IRGC). 2008a. Risk governance guidelines for bioenergy policy. http://www.irgc.org/IMG/pdf/IRGC_ PB_Bioenergy_WEB-2.pdf

International Risk Governance Council (IRGC). 2008b. Synthetic Biology: Risks and Opportunities of an Emerging Field. IRGC, Geneva, Switzerland.

International Water and Sanitation Centre (IWSC). 1988. Community Participation and Women's Involvement in Water Supply and Sanitation Projects: a compendium paper. IWSC, The Hague, Netherlands.

Isenberg, A.C. (ed.). 2006. The Nature of Cities: Culture, Landscape and Urban Space. University of Rochester Press, Rochester, NY, USA.

IUCN SSC Medicinal Plant Specialist Group. 2007. International Standard for Sustainable Wild Collection of Medicinal and Aromatic Plants (ISSC-MAP). Version 1.0. Bundesamt für Naturschutz (BfN), Bonn, Germany, MPSG/SSC/IUCN, Gland, Switzerland, WWF Germany, Frankfurt, Germany, and TRAFFIC, Cambridge, UK. BfN-Skripten 195. http://www.floraweb.de/MAP-pro/ Standard_Version1_0.pdf

IUCN, UNEP and WWF. 1980. Stratégie mondiale de la conservation : la conservation des ressources vivantes au service du développement durable. IUCN, Gland, Switzerland.

IUCN, UNEP and WWF. 1991. Sauver la planète : stratégie pour l'avenir de la vie.

IUCN. 2000a. Résolutions et recommandations. IUCN, Gland, Switzerland and Cambridge, UK. vii +76 pp.

IUCN. 2000b. Déclaration de principes de l'UICN 
sur l'utilisation durable des ressources biologiques sauvages. Résolution 2.29 adoptée lors du Congrès mondial de la nature, Amman, Octobre 2000. http://intranet.iucn.org/ webfiles/doc/SSC/SSCwebsite/SUSG_policy_ fr.pdf

IUCN. 2001. Transboundary Protected Areas for Peace and Cooperation. IUCN, Gland, Switzerland.

IUCN. 2003. L'Accord de Durban. http://cmsdata. iucn.org/downloads/durbanaccordfr.pdf

IUCN. 2008a. Biodiversité : mon hôtel agit. Guide pour une utilisation durable des ressources biologiques. IUCN, Gland, Switzerland. 128 pp.

IUCN. 2008b. Drylands - an economic asset for rural liveliboods and economic growth. Drylands Challenge Paper. http://cmsdata.iucn. org/downloads/draft_drylands_challenge_ paper_29sept08.pdf

IUCN. 2008c. Failure to act will push bluefin tuna fishery to extinction says IUCN. http://cms.iucn. org/about/work/programmes/marine/marine_ news/?uNewsID $=2343$

IUCN. 2008d. The 2008 IUCN Red List of Threatened Species. www.iucnredlist.org

IUCN. 2008e. WCC Résolution 4.056 - Stratégies de conservation fondées sur les droits. IUCN Resolutions and Recommendations, World Conservation Congress, Barcelona 5-14 October 2008.

IUCN. 2009a. Lignes directrices opérationnelles sur l'engagement auprès du secteur privé. http://cmsdata.iucn.org/downloads/fr_ operational_guidelines_for_private_sector_ engagement.pdf

IUCN. 2009b. The Future of the World Heritage Convention, Challenges for the next twenty years: An IUCN Perspective. IUCN, Gland, Switzerland.
IWMI. 2009. Projected water scarcity in 2025. http:// www.lk.iwmi.org/Press/map0.htm (last accessed on 30 April 2009).

Iza, A. and R. Stein (eds.). 2009. Rule: reforming water governance. IUCN, Gland, Switzerland.

Jackson, D.L. and L.L. Jackson (eds.). 2002. The Farm as Natural Habitat: Reconnecting Food Systems with Ecosystems. Island Press, Washington DC, USA.

Jacobson, M.F. 2004. Liquid candy: How soft drinks are harming Americans' health. Center for Science in the Public Interest, Washington DC, USA. 15 October 2004. www.cspinet.org/ sodapop/liquid_candy.htm

James, A.N., K.J. Gaston and A. Balmford. 2001. Can we afford to conserve biodiversity? BioScience 51: 43-52.

Janki, M. and C. Sose. 2008. The Wai Wai Protected Area - Our Land: Our Life. Governance for Sustainability - Issues, Challenges, Successes. 123-132. In Bosselmann, K., R. Engel and P. Taylor (eds). IUCN, Gland, Switzerland. xvi +260 pp.

Jianchu, X. (ed.). 2000. Links between Cultures and Biodiversity. Yunnan Science and Technology Press, Kunming, China.

Kearney, M., R. Shine and W.P. Porter. 2009. The potential for behavioral thermoregulation to buffer "cold-blooded" animals against climate warming. Proceedings of the National Academy of Sciences 106: 3835-3840. doi: 10.1073/ pnas.0808913106

Kelly, A.E. and M.L. Goulden. 2008. Rapid shifts in plant distribution with recent climate change. Proceedings of the National Academy of Sciences 105: 11823-11826. doi: 10.1073/pnas.0802891105

Klare, M.T. 2001. Resource Wars: The New Landscape of Global Conflict. Metropolitan Books, New York, USA. 
Klee, G.A. (ed.). 1980. World Systems of Traditional Resource Management. Edward Arnold, London, UK.

Kousky, C. 2005. Choosing from the policy toolbox. Ecosystem Marketplace. http://ecosystem marketplace.com/pages/article.opinion. php?component_id=4002\&component_ version_id=5679\&language_id $=12$

Kunz, T.H., E.B. Arnett, B.M. Cooper, W.P. Erickson, R.P. Larkin, T. Mabee, M.L. Morrison, M.D. Strickland and J.M. Szewczak. 2007a. Assessing Impacts of Wind-Energy Development on Nocturnally Active Birds and Bats: A Guidance Document. Journal of Wildlife Management 71(8): 2449-2486. doi: 10.2193/2007-270

Kunz, T.H., E.B. Arnett, W.P. Erickson, A.R. Hoar, G.D. Johnson, R.P. Larkin, M.D. Strickland, R.W. Thresher and M.D. Tuttle. 2007b. Ecological impacts of wind energy development on bats: questions, research needs, and hypotheses. Frontiers in Ecology and Environment 5(6): 315-324.

Kuo, F.E., M. Bacaicoa and W. Sullivan. 1998. Transforming inner-city landscapes: Trees, sense of safety and preference. Environment and Behavior 30(1): 28-59.

Laffoley, D. d'A. (ed.). 2008. Towards Networks of Marine Protected Areas. IUCN, Gland, Switzerland.

Langhammer, P.F., M.I. Bakarr, L.A. Bennun, T.M. Brooks, R.P. Clay, W. Darwall, N. De Silva, G.J. Edgar, G. Eken, L.D.C. Fishpool, G.A.B. da Fonseca, M.N. Foster, D.H. Knox, P. Matiku, E.A. Radford, A.S.L. Rodrigues, P. Salaman, W. Sechrest and A.W. Tordoff. 2007. Identification and Gap Analysis of Key Biodiversity Areas: Targets for Comprehensive Protected Area Systems. IUCN, Gland, Switzerland.

Lavorel, S. 1998. Mediterranean terrestrial ecosystems: research priorities on global change effects. Global Ecology and Biogeography 7: 157-166.

Liu, J., G.C. Daily, P.R. Ehrlich and G.W. Luck. 2003. Effects of household dynamics on resource consumption and biodiversity. Nature 421: 530-533.

Louisiana Department of Agriculture and Forestry. 2005. Imposition of Quarantine. Office of Agricultural and Environmental Sciences. http://www.ldaf.state.la.us/portal/Portals/0/ AES/Horticulture/katrinaquarantine.pdf

Louv, R. 2005. The Last Child in the Woods: Saving our children from nature-deficit order. Algonguin Books, New York, USA.

Lyon, T.P. and J.W. Maxwell. 2008. Corporate Social Responsibility and the Environment: A Theoretical Perspective. Review of Environmental Economics and Policy 1: 1-22. doi: 10.1093/reep/ren004

Mace, G.M. and E.J. Hudson. 1999. Attitudes toward Sustainability and Extinction. Conservation Biology 13: 242-246.

Mack, R.N., D. Simberloff, W.M. Lonsdale, H. Evans, M. Clout and F.A. Bazzaz. 2000. Biotic invasions: Causes, epidemiology, global consequences, and control. Ecological Applications 10: 689-710.

MacLeod, C.E. 2008. It Takes a Village to Save the Polar Bear. Governance for Sustainability - Issues, Challenges, Successes. 141-148. In Bosselmann, K., R. Engel and P. Taylor (eds). IUCN, Gland, Switzerland. xvi +260 pp.

Maffi, L. 2005. Linguistic, Cultural, and Biological Diversity. Annual Review of Anthropology 29: 599-617, doi: 10.1146/annurev. anthro.34.081804.120437

Magrin, G., C. Gay García, D. Cruz Choque, J.C. Giménez, A.R. Moreno, G.J. Nagy, C. Nobre and A. Villamizar. 2007. Latin America. Climate Change 2007: Impacts, Adaptation and 
Vulnerability. Contribution of Working Group II to the Fourth Assessment Report of the Intergovernmental Panel on Climate Change. 581-615. In Parry, M.L., O.F. Canziani, J.P. Palutikof, P.J. van der Linden and C.E. Hanson (eds.). Cambridge University Press, Cambridge, UK.

Malthus, T. 1798. An Essay on the Principle of Population. J. Johnson, London, UK.

Margolis, J.D., H.A. Elfenbein and J.P. Walsh. 2007. Does it pay to be good? A meta-analysis and redirection of research on the relationship between corporate social performance and financial performance. (Mimeograph). http:// stakeholder.bu.edu/2007/Docs/Walsh,\%20 Jim $\% 20$ Does $\% 20$ It $\% 20$ Pay $\% 20$ to\%20Be $\% 20$ Good.pdf

Massachusetts Institute of Technology (MIT). 2007. The future of geothermal energy. http:// geothermal.inel.gov/publications/future_of_ geothermal_energy.pdf

Matthews, H.D. and K. Caldeira. 2007. Transient climate-carbon simulations of planetary geoengineering. Proceedings of the National Academy of Sciences 104: 9949-9954.

May, R.M. 1999. Conservation: Dealing with Extinction. Imagine Tomorrow's World. 57-76. In McNeely, J.A. (ed.). IUCN, Gland, Switzerland.

McCann, K. 2000. The Diversity-Stability Debate. Nature 405: 228-233.

McCauley, D. 2006. Selling out on Nature. Nature 443: 27-28.

McDonough, W. and M. Braungart. 2002. Cradle to cradle: remaking the way we make things. North Point Press, New York, USA. 194 pp.

McKibben, B. 2007. Deep Economy: The Wealth of Communities and the Durable Future. Henry Holt, New York, USA.
McNeely, J.A. 1988. Economics and Biological Diversity: Developing and Using Economic Incentives to Conserve Biological Resources. IUCN, Gland, Switzerland.

McNeely, J.A. and P. Wachtel. 1988. Soul of the Tiger. Doubleday, New York, USA.

McNeely, J.A., H.A. Mooney, L.E. Neville, P. Schei and J.K. Waage (eds.). 2001. A Global Strategy on Invasive Alien Species. IUCN, Gland, Switzerland.

McNeely, J.A. 2002. The role of taxonomy in conserving biodiversity. Journal of Nature Conservation 10(3): 145-154.

McNeely, J.A. and S.J. Scherr. 2003. Ecoagriculture: Strategies for Feeding the World and Conserving Wild Biodiversity. Island Press, Washington DC, USA.

McNeely, J. 2008. Applying the Diversity of International Conventions to Address the Challenges of Climate Change. Michigan State Journal of International Law 17: 123-137.

McNeely, J. et al. 2009. The Wealth of Nations. Conservation International, Arlington, VA, USA, and Cemex, San Pedro Garza García, Mexico.

McNeely, J.A. In press. Conservation and conflict. State of the Wild 2010. Wildlife Conservation Society, New York, USA.

Meadows, D.H., D.L. Meadows, J. Randers and I. Behrens. 1972. The Limits to Growth. Universe Books, New York, USA.

Meffe, G.K., C.R. Carroll and M.J. Groom. 2005. What is Conservation Biology? Principles of Conservation Biology. In Meffe, G.K., C.R. Carroll and M.J. Groom (eds). Sinauer Associates, Sunderland, MA, USA.

Meilleur, B. and T. Hodgkin. 2004. In situ conservation of crop wild relative: status 
and trends. Biodiversity and Conservation 13: 663-684.

Melnick, D., J.A. McNeely, Y. Kakabadse Navarro, G. Schmidt-Traub and R.R. Sears. 2005. Environment and Human Well-Being: A Practical Strategy. UN Millennium Project. Task Force on Environmental Sustainability. Earthscan, London, UK and Sterling, VA, USA.

Merode, de E. and G. Cowlishaw. 2006. Species protection, the changing informal economy, and the politics of access to the bush meat trade in the Democratic Republic of Congo. Conservation Biology 20(4): 1262-1271.

Milledge, S.A.H., I.K. Gelvas and A. Ahrends. 2007. Forestry, Governance and National Development: Lessons Learned From a Logging Boom in Southern Tanzania. TRAFFIC East / Southern Africa, Dar Es Salaam, Tanzania.

Millennium Ecosystem Assessment (MA). 2003. Ecosystems and Human Well-Being: A Framework for Assessment. Island Press, Washington DC, USA.

Millennium Ecosystem Assessment (MA). 2005a. Desertification synthesis. www.maweb.org/ documents/document.355.aspx.pdf

Millennium Ecosystem Assessment (MA). 2005b. Ecosystems and Human Well-Being: Biodiversity Synthesis. World Resources Institute, Washington DC, USA.

Millennium Ecosystem Assessment (MA). 2005c. Ecosystems and Human Well-Being: Wetlands and Water Synthesis. World Resources Institute, Washington DC, USA. http:// www.millenniumassessment.org/documents/ document.358.aspx.pdf

Millennium Ecosystem Assessment (MA). 2005d. Synthesis. http://www.millenniumassessment. org/documents/document.356.aspx.pdf

Milner-Gulland, E.J., E.L. Bennett and SCB 2002 Annual Meeting Wild Meat Group. 2003.
Wild meat: the bigger picture. Trends in Ecology \& Evolution 18: 351-357.

Mohammed, A.G. 2008. Gum Arabic Belt Potential in the Livelihood improvement of Central Sudan Drylands. 4th World Conservation Congress. Barcelona. Alliances Workshop.

Mortimore, M., S. Anderson, L. Cotula, K. Faccer, C. Hesse, A. Mwangi, W. Nyangena and J. Skinner. 2008. Drylands - An Economic Asset for Rural Livelihoods and Economic Growth. Draft Challenge Paper of IUCN, IIED and UNDP/DDC. http://cmsdata.iucn. org/downloads/draft_drylands_challenge_ paper_29sept08.pdf

Myers, N. and J.L. Simon. 1994. Scarcity or Abundance? A debate on the environment. W.W. Norton, New York, USA. xix + 254 pp.

Naidoo, R., A. Balmford, R. Costanza, B. Fisher, R.E. Green, B. Lehner, T.R. Malcolm and T.H. Ricketts. 2008. Global Mapping of Ecosystem Services and Conservation Priorities.

Proceedings of the National Academy of Sciences of the United States 105: 9495-9500.

Narayan, D. 1995. Contribution of People's Participation: Evidence from 121 Rural Water Supply Projects. The World Bank, Washington DC, USA.

National Geographic. 2009. Greendex 2009: Consumer Choice and the Environment - A Worldwide Tracking Survey Highlights Report. http://www.nationalgeographic.com/ greendex/assets/Greendex_Highlights_ Report_May09.pdf

National Research Council (NRC). 2007. Environmental Impacts of Wind Energy Projects. http://books.nap.edu/openbook.php?record_ $\mathrm{id}=11935$ \&page $=7$

Navarro, E., A. Baun, R. Behra, N.B. Hartmann, J. Filser, A.J. Miao, A. Quigg, P.H. Santschi, and L. Sigg. 2008. Environmental behavior and ecotoxicity of engineered nanoparticles 
to algae, plants, and fungi. Ecotoxicology 17:

372-386. doi: 10.1007/s10646-008-0214-0

NBSAP Bhutan. 2002. Biodiversity Action Plan for

Bhutan. Government of Bhutan, Thimphu, Bhutan.

NBSAP Yemen. 2005. National Biodiversity Strategy and Action Plan, 16. Ministry of Environment and Water, Yemen.

Nuñez-Iturri, G. and H.F. Howe. 2007. Bushmeat and the Fate of Trees with Seeds Dispersed by Large Primates in a Lowland Rain Forest in Western Amazonia. Biotropica 39(3): 348-354. doi: 10.1111/j.1744-7429.2007.00276.x

Oates, J.F. 1998. Myth and Reality in the Rain Forest. University of California Press, Berkeley, USA.

Organisation for Economic Co-operation and

Development (OECD). 2008. Ressources naturelles et croissance pro-pauvres : Enjeux économiques et politiques. DAC Guidelines and Reference Series. OECD, Paris, France.

Organisation for Economic Co-operation and Development (OECD). 2009. L'aide au développement en 2008 à son plus haut niveau. http://www.oecd.org/document/35/0,3343, fr_2649_34487_42461389_1_1_1_1,00.html

Organisation for Economic Co-operation and Development/International Energy Agency (OECD/IEA). 2006. World Energy Outlook 2006. OECD, Paris, France.

Orr, D. 2005. Armageddon versus extinction. Conservation Biology 19: 290-292.

Osofsky, S.A., S. Cleaveland, W.B. Karesh, M.D. Kock, P.J. Nyhus, L. Starr and A. Yang (eds.). 2005. Conservation and Development Interventions at the Wildlife/Livestock Interface: Implications for Wildlife, Livestock and Human Health. IUCN, Gland, Switzerland and Cambridge, UK. xxxiii + 220 pp. http://data. iucn.org/dbtw-wpd/edocs/SSC-OP-030.pdf
Oviedo, G. 2006. Land Ownership Issues in Forest Restoration. Forest Restoration in Landscapes Beyond Planting Trees. 84-94. In Mansourian, S., E. Vallauri and N. Dudley (eds). Springer - WWF, New York, USA.

Palsbøll, P.J., M. Berube, H.J. Skaug and C. Raymakers. 2006. DNA Registers of Legally Obtained Wildlife and Derived Products as Means to Identify Illegal Takes. Conservation Biology 20: 1284-1293. doi: 10.1111/j.15231739.2006.00429

Parmesan, C. and G. Yohe. 2003. A globally coherent fingerprint of climate change impacts across natural systems. Nature 421: 37-42.

Patz, J., P. Daszak, G.M. Tabor, A. Alonso Aguirre, M. Pearl, J. Epstein, N.D. Wolfe, A. Marm Kilpatrick, J. Foufopoulos, D. Molyneux, D.J. Bradley and Members of the Working Group on Land Use Change and Disease Emergence. 2004. Unhealthy Landscapes: Policy Recommendations on Land Use Change and Infectious Disease Emergence. Environmental Health Perspectives 112(10): 1092-1098. doi: 10.1289/ehp.6877

Perrings, C. 2006. Resilience and sustainable development. Environment and Development Economics 11: 417-427.

Perry, A.L., P.J. Low, J.R. Ellis and J.D. Reynolds. 2005. Climate Change and Distribution Shifts in Marine Fishes. Science 308: 1912-1915.

Pinter, L. 2006. International Experience in Establishing Indicators for the Circular Economy and Considerations for China. Report for the Environment and Social Development Sector Unit, East Asia and Pacific Region, The World Bank. http://www.iisd.org/pdf/2006/measure_ circular_economy_china.pdf

Pirages, D. and T. De Geest. 2003. Ecological Security: An Evolutionary Perspective on Globalization.

Rowman and Littlefield, Lanham, MD, USA. 
Poertner, H.O. and R. Knust. 2007. Climate change affects marine fishes through the oxygen limitation of thermal tolerance. Science 315: 95-97.

Polasky, S., E. Nelson, J. Camm, B. Csuti, P. Fackler, E. Lonsdorf, C. Montgomery, D. White, J. Arthur, B. Garber-Yonts, R. Haight, J. Kagan, A. Starfield and C. Tobalske. 2008. Where to Put Things? Spatial Land Management to Sustain Biodiversity and Economic Returns. Biological Conservation 141: 1505-1524.

Posey, D.A. (ed.). 1999. Cultural and Spiritual Values of Biodiversity. United Nations Environment Programme, Nairobi, Kenya.

Pounds J.A., M.P.L. Fogden and J.H. Campbell. 1999. Biological response to climate change on a tropical mountain. Nature 398: 611-615.

Pounds, J.A., M.R. Bustamante, L.A. Coloma, J.A. Consuegra, M.P.L. Fogden, P.N. Foster, E. La Marca, K.L. Masters, A. Merino-Viteri, R. Puschendorf, S.R. Ron, G.A. SanchezAzofeifa, C.J. Still and B.E. Young. 2006. Widespread amphibian extinctions from epidemic disease driven by global warming. Nature 469: 161-167.

Poverty Environment Partnership (PEP). 2005. Case studies on pro-poor growth: Diamond-based growth in Botswana. http:// povertyenvironment.net/files/CASE $\% 20$ Botswana.pdf

Pressey, R.L., M. Cabeza, M.E. Watts, R.M. Cowling and K.A. Wilson. 2007. Conservation planning in a changing world. Trends in Ecology \& Evolution 22: 583-592.

Pretty, J.N. (ed). 2005. The Earthscan Reader in Sustainable Agriculture. Earthscan, London, UK.

Redford, K. and E. Fearn (eds.). 2007. Protected Areas and Human Liveliboods. Wildlife Conservation society, New York, USA.
Reid, Walter, et al. 2006. Nature: the many benefits of ecosystem services. Nature 443: 749.

Rivalan, P., V. Delmas, E. Angulo, L.S. Bull, R.J. Hall, F. Courchamp, A.M. Rosser and N. Leader-Williams. 2007. Can bans stimulate wildlife trade? Nature 447: 529-530.

Rodrigues, A.S.L., S.J. Andelman, M.I. Bakarr, L. Boitani, T.M. Brooks, R.M. Cowling, L.D.C. Fishpool, G.A.B. da Fonseca, K.J. Gaston, M. Hoffmann, J.S. Long, P.A. Marquet, J.D. Pilgrim, R.L. Pressey, J. Schipper, W. Sechrest, S.N. Stuart, L.G. Underhill, R.W. Waller, M.E.J. Watts and X. Yan. 2004. Effectiveness of the global protected area network in representing species diversity. Nature 428: 640-643.

Rodriguez, G., M. Blanco and F. Azofeifa. 2004. Diversity Makes the Difference. IUCN, San Jose, Costa Rica.

Roe, D. 2008. The origins and evolution of the conservation-poverty debate: a review of key literature, events and policy processes. Oryx 42(4): 491-503. 1/1-2: 115-132. doi: 10.1111/j.1749-818X.2007.00004.x

Ron, S.R., W.E. Duellman, L.A. Coloma and M.R. Bustamante. 2003. Population decline of the Jambato Toad Atelopus ignescens (Anura: Bufonidae) in the Andes of Ecuador. J. Herpetol. 37: 116-126.

Roundtable for Sustainable Biofuels (RSB). 2008. Global principles and criteria for sustainable biofuels production Version Zero. http://cgse.epfl.ch/ webdav/site/cgse/shared/Biofuels/VersionZero/ Version\%20Zero_RSB_Std_en.pdf

Royal Society of Chemistry (RSC). 2008. China quake hits chemical industry. http://www.rsc.org/ chemistryworld/News/2008/May/16050802.asp

Rubinoff, D. 2006. Utility of Mitochondrial DNA Barcodes in Species Conservation. Conservation Biology 20: 1026-1033. doi: 10.1111/j.1523-1739.2006.00372.x 
Sahgal, B. 2005. Kids for Tigers: A globally replicable school contact programme to win support for wildlife and protected areas. The Urban Imperative. 121-123. In Tryzna, T. (ed.). California Institute of Public Affairs, Sacramento, CA, USA.

Sala O.E., F.S. Chapin, J.J. Armesto et al. 2000. Global biodiversity scenarios for the year 2100. Science 287: 1770-1774.

Sandwith, T., C. Shine, L. Hamilton and D. Sheppard. 2001. Transboundary Protected Areas for Peace and Co-operation. IUCN, Gland, Switzerland and Cambridge, UK.

Sayer, J., B. Campbell, L. Petheram, M. Aldrich, M.R. Perez, D. Endamana, Z.N. Dongmo, L. Defo, S. Mariki, N. Doggart and N. Burgess. 2007. Assessing environment and development outcomes in conservation landscapes. Biodiversity Conservation 16: 2677-2694.

Sayer, J.A. and S. Maginnis. 2005. Forests in landscapes: expanding horizons for ecosystem forestry. Forests in Landscapes: ecosystem approaches to sustainability. In Sayer, J. and S. Maginnis (eds.). Earthscan, London, UK. 257 pp.

Scherl, L., A. Wilson, R. Wild, J. Blockhus, P. Franks, J. McNeely and T. McShane. 2004. Can Protected Areas Contribute to Poverty Reduction? Opportunities and Limitations. IUCN, Gland, Switzerland.

Scherr, S. 1999. Poverty-Environment Interactions in Agriculture: Key Factors and Policy Implications. Poverty and Environment Initiative Background Paper 3. UNDP, New York, USA.

Scherr, S.J. and J.A. McNeely (eds.). 2007. Farming with Nature: The Science and Practice of Ecoagriculture. Island Press, Washington DC, USA

Schippmann, U., D. Leaman and A.B. Cunningham. 2006. A comparison of cultivation and wild collection of medicinal and aromatic plants under sustainability aspects. Medicinal and Aromatic Plants. In Bogers, R.J., L.E. Craker and D. Lange (eds.).Springer, Dordrecht, The Netherlands. pp. 75-95.

Scholte, P., S. Kari, M. Moritz and H. Prins. 2006. Pastoralist Responses to Floodplain Rehabilitation in North Cameroon. Human Ecology 34: 27-51. doi: 10.1007/s10745-005-9001-1

Schroth, G. and C.A. Harvey. 2007. Biodiversity conservation in cocoa production landscapes: An overview. Biodiversity and Conservation 16(8): 2237-2244.

Schuyt, K. and L. Brander. 2004. Living Waters Conserving the source of life. The economic value of the world's wetlands. WWF, Gland, Switzerland.

Schwanz, L.E. and F.J. Janzen. 2008. Climate Change and Temperature-Dependent Sex Determination: Can Individual Plasticity in Nesting Phenology Prevent Extreme Sex Ratios? Physiological and Biochemical Zoology 81(6): 826-834. doi: 10.1086/590220

Schwarz, M.W., J. Thorne and J. Viers. 2006. Biotic homogenization of the California flora in urban and urbanizing regions. Biological Conservation 127: 282-291.

Sheil, D. and D. Murdiyarso. 2009. How Forests Attract Rain: An Examination of a New Hypothesis. BioScience 59: 341-347. doi: 10.1525/bio.2009.59.4.12.

Shiro, W. 2004. Enclose cities in nature-developing new technologies to build cities within nature. City Planning Revierw 249: 49-54.

Smith, D.M. and S. Barchiesi. 2008. Environment as infrastructure: Resilience to climate change impacts on water through investments in nature. Perspectives on water and climate change adaptation. http:// cmsdata.iucn.org/downloads/iucnperspap_ environment_as_infrastructure_1.pdf 
Smith, H.O., C.A. Hutchison, C. Pfannkoch and J.C. Venter. 2003. Generating a synthetic genome by whole genome assembly: $\varphi \mathrm{X} 174$ bacteriophage from synthetic oligonucleotides. Proceedings of the National Academy of Sciences 100: 15440-15445. doi: 10.1073/pnas.2237126100

Smith, K.G. and W.R.T. Darwall (compilers). 2006. The Status and Distribution of Freshreater Fish Endemic to the Mediterranean Basin. IUCN Red List of Threatened Species - Mediterranean Regional Assessment No.1. IUCN, Gland Switzerland and Cambridge, UK.

Smith, M., D. de Groot and G. Bergkamp. 2006. Pay: Establishing payments for watershed services. IUCN, Gland, Switzerland.

Sobrevila, C. 2008. The Role of Indigenous Peoples in Biodiversity Conservation: The Natural but Often Forgotten Partners. A report for the World Bank. http://siteresources. worldbank.org/INTBIODIVERSITY/ Resources/RoleoflndigenousPeoplesin BiodiversityConservation.pdf.

Solomon, S., G.K. Plattner, R. Knutti and P. Friedlingstein. 2009. Irreversible climate change due to carbon dioxide emissions. Proceedings of the National Academy of Sciences 106: 1704-1709. doi: 10.1073/ pnas.0812721106

Srinivasan, U.T., S.P. Carey, E. Hallstein, P.A.T. Higgins, A.C. Kerr, L.E. Koteen, A.B. Smith, R. Watson, J. Harte and R.B. Norgaard. 2008. The debt of nations and the distribution of ecological impacts from human activities. Proceedings of the National Academy of Sciences 105(5): 1768-1773. doi: 10.1073/ pnas.0709562104

Stern, N. 2006. Stern Review on the Economics of Climate Change. Cambridge University Press, Cambridge, UK. http://www.hm-treasury.gov. uk/stern_review_report.htm
Stiling, P. 2002. Potential non-target effects of a biological control agent, prickly pear moth, Cactoblastis cactorum (Berg) (Lepidoptera: Pyralidae) in North America and possible management actions. Biological invasions 4: 273-281.

Stuart S.N., G.W. Archibald, J. Ball, R.J. Berry, S.D. Emmerich, D.M. Evans, J.R. Flenley, K.J. Gaston, D.R. Given, A.G. Gosler, P. Harris, J. Houghton, E.D. Lindquist, D.C. Mahan, M.D. Morecroft, D.C. Moyer, D. Murdiyarso, B.W.W. Musiti, C. Nicolson, A. Oteng-Yeboah, A.J. Plumptre, G. Prance, V. Ramachandra, J.B. Sale, J.K. Sheldon, S. Simiyu, R. Storey, L.G. Underhill, J. Vickery and T. Whitten. 2005. Conservation theology for conservation biologists - A reply to David Orr. Conservation Biology 19: 1689-1692.

Sumaila, U.R. and D. Pauly. 2006. Catching More Bait: A Bottom-Up Re-Estimation Of Global Fisheries Subsidies (2nd edition). Fisheries Centre Research Reports Volume 14 Number 6. http://www.fisheries.ubc.ca/archive/ publications/reports/14-6.pdf

Sutherland, W.J. et al. 2008. Future novel threats and opportunities facing UK biodiversity identified by horizon scanning. Journal of Applied Ecology 45: 821-833. doi: 10.1111/j.1365-2664.2008.01474.x

ten Brink, P. 2008. Loss of ecosystem services from land-based ecosystems. COPI Study. In Braat, L. and P. ten Brink (eds.)

The Economics of Ecosystems and Biodiversity (TEEB). 2008. Interim report on The Economics of Ecosystems and Biodiversity (2008). http:// ec.europa.eu/environment/nature/ biodiversity/economics/

The Economist. 2009. Charlemagne: Fishy Tales: 25 April 2009.

The Trust for Public Land. (2009). http://www.tpl.org/ 
Thomas, C.D. and J.J. Lennon. 1999. Birds extend their ranges northwards. Nature 399: 213.

Thomas, L. and J. Middleton. 2003. Guidelines for Management Planning of Protected Areas. IUCN, Gland, Switzerland and Cambridge, UK. ix +79 pp.

Thouless, C. 2008. Human Wildlife ConflictBiology and Beyond. World Conservation Forum Workshop Report, Event 1537. http://intranet.iucn.org/webfiles/ftp/ public/ForumEvents/E1537/Final\%20 Document/1537_REPORT_Thouless_C_ Human $\% 20$ Wildlife $\% 20$ Conflict $\% 20$ Biology $\% 20$ and $\% 20$ Beyond.pdf

TRAFFIC. 2008. What's Driving the Wildlife Trade? A Review of Expert Opinion on Economic and Social Drivers of the Wildlife Trade and Trade Control Efforts in Cambodia, Indonesia, Lao PDR and Vietnam. East Asia and Pacific Region Sustainable Development Discussion Papers. East Asia and Pacific Region Sustainable Development Department, World Bank, Washington, DC, USA.

Tumpey, T.M. et al. 2005. Characterization of the reconstructed 1918 Spanish influenza pandemic virus. Science 310: 77-80.

Turner, W., T. Nakamura and M. Dinetti. 2004. Global urbanization and the separation of humans from nature. Bioscience 54(6): 1-6.

Turner, W.R., K. Brandon, T.M. Brooks, R. Costanza, G.A.B. da Fonseca and R. Portela. 2007. Global Conservation of Biodiversity and Ecosystem Services. BioScience 57: 868-873.

UN Convention to Combat Desertification (UNCCD). www.unccd.int/knowledge/ menu.php

UN Water. 2007. Coping with water scarcity: challenge of the twenty-first century. Prepared for World Water Day 2007. http://www.unwater.org/ wwd07/downloads/documents/escarcity.pdf
UN. 1982. The World Charter for Nature. UN GA RES 37/7. http://sedac.ciesin.columbia.edu/ entri/texts/world.charter.for.nature.1982.html

UNESCO. 2006. Décisions adoptées lors de la 30e session $d u$ Comité du patrimoine mondial. http://whc. unesco.org/archive/2006/whc06-30com-19f.pdf

UNESCO. 2009. Liste du patrimoine mondial en péril. http://whc.unesco.org/fr/peril/

United Nations (UN). 2008. Objectifs du Millénaire pour le développement 2008. UN, New York, USA.

United Nations Department of Economic and Social Affairs (UN DESA). 2009. World Population Prospects-The 2008 Revision. http:// www.un.org/esa/population/publications/ wpp2008/wpp2008_highlights.pdf

United Nations Development Programme (UNDP). 2002. Poverty and Environment Initiative. UNDP, New York, USA.

United Nations Development Programme (UNDP). 2004. Gender \& Energy for sustainable development: a Toolkit and Resource Guide. http://www.undp.org/energy/genenergykit/

United Nations Development Programme (UNDP). 2005. Concept paper: High-Level Commission on Legal Empowerment of the Poor: Poverty Reduction through Improved Asset Security, Formalisation of Property Rights and the Rule of Law. http://www.undp.org/ legalempowerment/pdf/Concept_Paper.pdf

United Nations Economic Commission for Europe (UNECE) and FAO. 2008. UNECE/ FAO Forest Products Annual Market Review, 2007-2008. Executive Summary. http:// timber.unece.org/fileadmin/DAM/ publications/executive-summary-2008.pdf

United Nations Environment Programme (UNEP). 2003. L'avenir de l'environnement mondial. http://www.unep.org/GEO/geo3/ french/pdf.htm 
United Nations Environment Programme (UNEP). 2005. After the Tsunami: Rapid Environmental Assessment. UNEP, Nairobi, Kenya. 140 pp.

United Nations Environment Programme (UNEP). 2006. Global International Waters Assessment: Challenges to International Waters: Regional Assessments in a Global Perspective. UNEP, Nairobi, Kenya. http://www.unep. org/dewa/giwa/publications/finalreport/ executive_summary.pdf

United Nations Environment Programme (UNEP). 2008a. Environment key to poverty reduction in Tanzania. The Environment Times \#4. http://www.grida.no/publications/et/ep4/ page/2641.aspx

United Nations Environment Programme (UNEP). 2008b. Environmental change and new infectious diseases. The Environment Times \#4. http://www.grida.no/publications/et/ep4/ page/2631.aspx

United Nations Millennium Project. 2005. Environment and Human Wellbeing: A practical strategy. Summary version of the report of the Task Force on Environmental Sustainability. The Earth Institute at Columbia University, New York, USA.

United Nations World Tourism Organisation (UNWTO). 2009. Bref apercu des grandes tendances. Baromètre OMT du tourisme mondial: janvier 2009. http://www.unwto.org/facts/eng/ pdf/barometer/UNWTO_Barom09_1_en_ excerpt.pdf

United Nations. 2008. Promotion and Protection of All Human Rights, Civil, Political, Economic, Social and Cultural Rights, Including the Right to Development: Human Rights and Climate change. Document A/HRC/7/L.21/Rev.1. http://daccessdds.un.org/doc/UNDOC/ LTD/G08/121/52/PDF/G0812152. pdf?OpenElement
Uphoff, N., A.S. Ball, E. Fernandes, H. Herren, O. Husson, M. Laing, C. Palm, J. Pretty, and P. Sanchez (eds.). 2006. Biological Approaches to Sustainable Soil Systems. CRC Press, Boca Raton, USA.

US Environmental Protection Agency (EPA). 2005. The Formosan Subterranean Termite in Georgia. http://www.caes.uga.edu/departments/ent/ upmp/termites.html

Valencia Declaration, a plea for the protection of marine biodiversity. http://www.marbef.org/ worldconference/declaration.php

Van Buskirk, J. and Y. Willi. 2004. Enhancement of Farmland Biodiversity within Set-Aside Land. Conservation Biology 18: 897-994. Based on a meta-analysis of 127 published studies.

Vasconcelos, P.F., A.P. Travassos da Rosa, S.G. Rodrigues, E.S. Travassos da Rosa, N. Dégallier, J.F. Travassos da Rosa. 2001. Inadequate management of natural ecosystem in the Brazilian Amazon region results in the emergence and reemergence of arboviruses. Cad Saude Publica 17 Suppl:155-64.

Vedeld, P., A. Angelsen, E. Sjaastad and G.K. Berg. 2004. Counting on the Environment: Forest Incomes and the Rural Poor. Environmental economics series PAPER NO. 98. http://www-wds.worldbank.org/servlet/ WDSContentServer/WDSP/IB/2004/09/3 0/000090341_20040930105923/Rendered/ PDF/300260PAPER0Counting0on0ENV0E DP0198.pdf

Veitch, C.R. and M.N. Clout. 2002. Turning the Tide: The eradication of invasive species. IUCN SSC Invasive Species Specialist Group. IUCN, Gland, Switzerland and Cambridge, UK. viii + 414 pp.

Victor, D., G. Morgan, J. Apt, J. Steinbrunner and K. Riche. 2009. The geoengineering option. Foreign Affairs 88(2): 69-76. 
Vié, J.-C., C. Hilton-Taylor and S.N. Stuart (eds.). 2009. Wildlife in a Changing World: An analysis of the 2008 IUCN Red List of Threatened Species. IUCN, Gland, Switzerland. 180 pp.

Wackernagel, M., N.B. Schulz, D. Deumling, A. Callejas Linares, M. Jenkins, V. Kapos, C. Monfreda, J. Loh, N. Myers, R. Norgaard and J. Randers. 2002. Tracking the Ecological Overshoot of the Human Economy. Proceedings of the National Academy of Sciences 99: 9266-9271.

Walther, G., E. Post, P. Convey, A. Menzel, C. Parmesan, T.J.C. Beebee, J.M. Fromentin, O. Hoegh-Guldberg and F. Bairlein. 2002. Ecological responses to recent climate Change. Nature 416: 389-395.

Wang, S.W. and D.W. McDonald. 2006. Livestock predation by carnivores in Jigme Singye Wangchuck National Park, Bhutan. Biological conservation 129: 558-565. doi: 10.1016/j. biocon.2005.11.024

Warren, M.S., J.K. Hill, J.A. Thomas, J. Asher, R. Fox, B. Huntley, D.B. Royk, M.G. Telferk, S. Jeffcoate, P. Hardingk, G. Jeffcoate, S.G. Willis, J.N. Greatorex-Daviesk, D. Mossk and C.D. Thomas. 2001. Rapid responses of British butterflies to opposing forces of climate and habitat change. Nature 414: 65-69.

Watson, A.M. 1983. Agricultural Innovation in the Early Islamic World. Cambridge University Press, Cambridge, UK.

Weizsäcker, E. and A. Lovins. 1995. Factor Four: Doubling Wealth, Halving Resource Use. Earthscan, London, UK.

White, R.P., S. Murray and M. Rohweder. 2000. Pilot analysis of global ecosystems: Grassland Ecosystems. World Resources Institute, Washington DC, USA.

WHO/IUCN/WWF. 1993. WHO/IUCN/WWF Guidelines on the Conservation of Medicinal Plants. IUCN, Gland, Switzerland.
Wild, R. and C. McLeod (eds.). 2008. Sacred Natural Sites: Guidelines for Protected Area Managers. IUCN, Gland, Switzerland.

Wilkinson, C. 2008. Status of the Coral Reefs of the World 2008. Global Coral Reef Monitoring Network and Rainforest Research Centre, Townsville, Australia. 296 pp.

Willer, H. and M. Minou Yussefi. 2006. The world of organic agriculture: Statistics and emerging trends. International Federation of Organic Agriculture Movements, Bonn, Germany and Research Institute of Organic Agriculture, Frick, Switzerland. http://orgprints. org/2555/01/willer-yussefi-2004-world-oforganic.pdf

Wilson, E.O. 1984. Biophilia. Harvard University Press, Cambridge, MA, USA.

Wittenberg, R. and M.J.W. Cock. 2001. Invasive alien species. How to address one of the greatest threats to biodiversity: a toolkit of best prevention and management practices. CAB International, Wallingford, Oxon, UK.

Woiwod, I.P. 1997. Review. Journal of Insect Conservation 1: 149-158.

World Bank. 2006. Strengthening Forest Law Enforcement and Governance: Addressing a Systemic Constraint to Sustainable Development. Report No. 36638-GLB. August 2006. http:// siteresources.worldbank.org/INTFORESTS/ Resources/ForestLawFINAL_HI_ RES_9_27_06_FINAL_web.pdf

World Bank. 2008. World Development Report 2008 Overview: Agriculture for Development. 2000/2001 World Development Report, World Bank, Washington DC. http:// www-wds.worldbank.org/external/default/ WDSContentServer/WDSP/IB/2007/11/ 13/000020953_20071113102401/Rendered/ PDF/414560ENGLISH018082137297501PU BLIC1.pdf 
World Bank. 2009. Water Resources management: transboundary waters. http://web.worldbank. org/WBSITE/EXTERNAL/TOPICS/EXTW AT/0,,contentMDK:21633352 menuPK:48 28556 pagePK:148956 piPK:216618 theS itePK:4602123,00.html (last accessed on 30 April 2009).

World Commission on Environment and Development (WCED). 1987. Our Common Future. WCED, New York, USA.

World Health Organization (WHO). 2002. Rapport sur la Santé dans le Monde 2002. www.who.org

World Health Organization (WHO). 2003. Directives OMS sur les bonnes pratiques agricoles et les bonnes pratiques de récolte (BPAR) relatives aux plantes médicinales. WHO, Geneva, Switzerland. http://whqlibdoc.who.int/ publications/2003/9242546275.pdf

World Resources Institute (WRI), IUCN and United Nations Environment Programme (UNEP). 1992. Stratégie mondiale de la biodiversité. World Resources Institute, Washington DC, USA.

World Resources Institute (WRI). 2008. Environmental Challenges after China's Sichuan Earthquake. http://earthtrends.wri.org/ updates/node/316

World Water Assessment Programme. 2009. Rapport mondial des Nations Unies sur la mise en valeur des ressources en eau 3: L'eau dans un monde qui change. UNESCO Publishing, Paris and Earthscan, London, UK.

World Wide Fund for Nature (WWF). 2007. WWF and Coca-Cola announce partnership to conserve freshwater resources. http://www.panda.org/ wwf_news/news/?uNewsID $=104940$

World Wide Fund for Nature (WWF). 2008. Living Planet Report 2008. http://assets.panda.org/ downloads/living_planet_report_2008.pdf
WorldWatch Institute. 2008. State of the World 2008: Innovations for a Sustainable Economy. WorldWatch Institute, Washington DC, USA. http://www.worldwatch.org/node/5568

Wunder, S. 2005. Payments for Ecosystem Services: Some nuts and bolts. CIFOR Occasional Paper 42: 1-24.

Zeidler, J. and J.K. Mulongoy. 2003. Dry and Sub-Humid Lands Programme of Work of the Convention on Biological Diversity: Connecting the CBD and the UN Convention to Combat Desertification. Reviere of European Community and International Environmental Law 12: 164-175. http://www. unccd.int/workshop/docs/ZeidlerArticle.pdf

Zeng, Q.T. and T. Tse. 2006. Exploring and Developing Consumer Health Vocabularies. Journal of the American Medical Informatics Association 13(1): 24-29. doi: 10.1197/jamia. M1761 


\section{Index}

accords commerciaux régionaux (ACR), 137

accords internationaux

action collective, 32

aide internationale, $135-137$

changements climatiques, 60-61

harmonisation, 134-135

respect des engagements, 136

accords multilatéraux sur l'environnement (AME), 133, 172

accords régionaux, 137, 138-139

adaptation écosystémique (EbA), 59-60

Afrique, faim et éco-agriculture, 21

Afrique du Sud, 179

Afrique sub-saharienne, 20, 88-89, 176

Agence européenne pour l'environnement (AEE), 120

agriculture. voir aussi systèmes agricoles

et biodiversité, 184, 186-189

changements climatiques, $187-188$

eau, 181, 183, 185

éco-agriculture, 21, 185

environnement et ressources, 21-22

espèces, 184, 189

femmes, 188-189

et forêts, 153

méthodes alternatives, 188

OGM, 129

reflet des écosystèmes naturels, 186-189

services écosystémiques, 45

zones arides, 168

aide au développement, 23, 135-137, 138

aires marines protégées (AMP), 66, 162, 163-164

aires protégées, 65-70

avenir, 69-70

changements climatiques, 57, 62

communautés locales, 41, 66-67

espèces non indigènes et envahissantes, 67

financement et capacités, 68-69

gestion, 66-68

lignes directrices, 66, 68 représentativité, 66

villes, 192-193

aires protégées transfrontalières, 96-98, 111-113

alimentation, sécurité, 21

Amazonie brésilienne, arbovirus, 109

animaux, et santé humaine, 109

Application des réglementations forestières et la gouvernance (FLEG), 154, 155

Approche écosystémique de la CDB, 38

aquaculture, 160

armées, et conservation, 98

Asie, 20, 88-89

Assemblée du millénaire des NU 2000, 179

Assemblée générale des Nations Unies, 48

Association internationale des hôtels et des restaurants, 144

Atlas climatique des oiseaux nicheurs d'Europe, 54

Au-delà de la REDD : le rôle des forêts dans le changement climatique, 156

autochtones, populations

et conservation, 40-43, 206

désertification, 169

droits, 40

participation à la gestion, 207

savoirs traditionnels, 39-40

Banque asiatique de développement (BAsD), 102

Banque de données mondiale 2008 sur les aires protégées, 66

Banque mondiale, 20, 156, 205

Barcelone, Forum de. voir Forum mondial de la nature bassins hydrographiques, 180-181, 185. voir aussi rivières et bassins

bétail, 111, 183

bien-être humain, dynamique du, 19-20

biocarburants, 85

biodiversité

et communications, 199-200

conservation, 18-19 
hauts-lieux, 27, 93

importance économique, 116-117

indicateurs, 17-18

objectifs 2010 et au-delà, $17-20$

perte de, 18-19

en tant que business, 144

thème du Forum de Barcelone, 16

Biodiversité : mon hôtel agit, 144

biodiversité culturelle, 39

bioéthique. voir éthique de la conservation

biologie synthétique, 130-131

biomasse, $85-86$

biomimétisme, 127-128

biotechnologies, 126-131

blanchiment écologique, 148

bois, coupes illégales, 154-155

Bonnes pratiques en matière d'agriculture et de collecte (GACP) de plantes médicinales, 111

Botswana, 119

braconnage, 95, 96

Brésil, 179, 187

Bureau du Haut Commissaire des Nations Unies pour les droits de l'homme, 60

Business and Biodiversity Offsets Programme (BBOP), 117

Business Development Facility, 156

cacao, 187

Cadre d'action de Hyogo pour 2005-2015, 61

capacité de charge de la planète, 33,120

capacité et financement, 68-69, 136

capital naturel, 119

carbone

et économie, 61-62, 117

forêts, 157

océans, 160

séquestration, 129

villes, 191

zones arides, 171

catastrophes naturelles et écosystèmes, 101-105

capacités, 103-104

changements climatiques futurs, 105

espèces envahissantes, 102-103

impacts, 103

planification de restauration, 102-105

support politique, 104

volet environnemental, 101-102

Centre du droit de l'environnement (CDE), 134-135, 205, 206
Centre international de l'eau et de l'assainissement (IRC), 180

certification, programme de, 118

chaîne logistique durable, 149

chaînes d'approvisionnement, 117

changements climatiques, 53-62

accords internationaux, 60-61

adaptation écosystémique, 59-60

agriculture, 187-188

aires protégées, 57,62

biodiversité, $54-57$

catastrophes naturelles, 105

eau, 181

écosystèmes, 56-57

espèces, 54-56, 161-162

forêts, $58,156-157$

îles, $57-58$

impacts sur l'homme, 59-60

océans, $159-160$

REDD, 58-59

services écosystémiques, 57

températures, 54-55

zones arides, 171

changements environnementaux, 19

changements universels, 16, 26, 201-203

Charte de la terre, 29, 32, 33

Charte mondiale de la nature, 29-31

chauves-souris, 87, 187

Chicago Wilderness, 194-195

Chine, 84, 120

Cible de Biodiversité 2010, 138

climat, 53-54, 130

Coca-Cola et WWF, 144

combustibles fossiles, alternatives, 84

Comité consultatif sur la baleine grise occidentale (WGWAP), 148

Comité du Patrimoine mondial de l'UNESCO, 57

commerce, 76, 77, 111, 137

Commission Brundtland, 134

Commission de sauvegarde des espèces (CSE), 56, 184

Commission économique des Nations Unies pour l'Europe (UNECE), 118

Commission européenne, 126, 154

Commission internationale pour la conservation des thonidés de l'Atlantique (CICTA), 161

Commission mondiale des aires protégées (CMAP) auto-gouvernance des autochtones, 41

culture et biodiversité, 39

gestion, 68

habitats marins, 66, 163

harmonisation des communications, 199 
jeunes, 70

villes, 192

Commission mondiale des barrages, 86

Commission sur le développement durable (CDD), 133

Commission UICN des politiques environnementales, économiques et sociales (CEESP), 123

communautés locales. voir aussi autochtones, populations

aires protégées, 41, 66-67

changements climatiques, 59-60

et conservation, 37, 40-43, 206

désertification, 169

gestion, 39-40, 207

compensations, 117

Compte à rebours 2010, 192

Conférence de Stockholm, 134

Conférence des Nations Unies sur le commerce et le développement (CNUCED), 136-137

Conférence des Nations Unies sur l'environnement et le développement, 48

conflits armés et conservation, 93-99

aires protégées transfrontalières, 96-98

biodiversité, 93

impacts sur la faune sauvage, 94-96

période post-conflit, problèmes, 96, 99

ressources naturelles, 93-94

conflits hommes-faune sauvage, 78

Congrès mondial de la nature $(\mathrm{CMN}), 10 \mathrm{n} 1$

Congrès mondial de la nature (CMN) 2000, 154

Congrès mondial de la nature $(\mathrm{CMN})$ 2008. voir aussi

Forum mondial de la nature

Centre de ressources, 10

espèces, 73

forêts, 152

océans, 162, 163

thèmes, 15-16

Congrès mondial des parcs de l'UICN, 40, 65, 70, 111

connaissances

espèces, 73-75

océans et conservation, 163-164

restauration post-catastrophes, 103

santé humaine, 108-109

zones arides, 171

connectivité, 68, 186

Conseil international de la gestion du risque (IRGC), 126

Conseil international des mines et métaux (CIMM), 143

Conseil international sur la gouvernance des risques, 86

Conseil mondial de l'énergie éolienne (GWEC), 87

Conseil mondial des affaires sur le développement durable (WBCSD), 145 conservation

feuille de route, 199-207

points clés pour l'avenir, 199

pratiques traditionnelles et modernes, 40-41

consommation et consommateurs

classement Greendex, 116

conséquences environnementales, 38

non durabilité, 115

programmes de certification, 118

Convention-cadre des Nations Unies sur les changements climatiques (CCNUCC), 54, 58, 60, 61, 134

Convention de Londres, 162-163

Convention des Nations Unies sur la lutte contre la désertification (UNCCD), 168-169, 171, 172

Convention des Nations Unies sur le droit de la mer (CNUDM-UNCLOS), 61

Convention du patrimoine mondial, 69, 134

Convention sur la diversité biologique (CDB)

accords, harmonisation, 134

adoption de la, 32

aires protégées, 67, 69

approche écosystémique, 38

biodiversité, valeur de la, 31

changements climatiques, 61

conflits armés, 98

conservation marine, 162

Convention des Parties, 133, 196, 203

espèces, 75

gouvernance, 204

Objectif Biodiversité 2010, 17

OGM, 129

savoirs et modes de vie traditionnels, 39

services écosystémiques, 48

soutien financier, 136

zones arides, 172

Convention sur le commerce international des espèces de faune et de flore sauvages menacées d'extinction (CITES), 77, 134

Convention sur les espèces migratrices (CMS), 61, 134

Convention sur les zones humides et la diversification (CCD), 61

coopération internationale, $133-140$

accords, 134-135, 138-139

avenir, $139-140$

cours d'eau, 180

mise en œuvre, 136-137

opinion publique, 138

volonté politique, 137-139

Costa Rica, 48-49, 51

Cradle to Cradle (McDonough et Braungart), 120 


\section{CRiSTAL, 60}

Critères mondiaux pour un tourisme durable, 144

culture

diversité de la, 39

lien à l'environnement, 202

services écosystémiques, 46, 47-48

valeur de la biodiversité, 50

déchets, 103, 124

Déclaration de 2007 des Nations Unies sur les droits des peuples autochtones, 40

Déclaration de Rio, 33

Déclaration de Stockholm, 29, 31, 60

Déclaration du millénaire, 23

déforestation, 58, 151, 153

démographie. voir population

désertification, 168-169, 171

développement

et environnement, 20-26, 138, 200

et forêts, 153

objectifs, 21-22, 23-26

ressources naturelles, 119

zones post-conflit, 96

développement durable, énergie, 88-90

Dialogue sur la forêt tropicale, 155

droits, 40, 60, 205-206

droits humains, principes du CDE, 206

durabilité

et économie, 115, 119-120

innovations, 203

intégration de l'environnement, 200-201

souci des entreprises, 145

Durban, Accord de, 40, 65, 68

eau. voir aussi systèmes d'eau douce

agriculture, 181, 183, 185

besoins de la nature, 178

changements climatiques, 181

consommation, 176-177

et économie, 86-87, 180

flux, 86, 104, 178

gestion, 178, 180, 181

gouvernance, 178-180

pénuries prévues, 176, 177

transfrontalière, 180

villes, 192-193

zones arides, 171-172

échelle des paysages, 104, 151-152, 178, 185

éco-agriculture, 21, 185

ECOLEX, 134-135 économie. voir aussi économie verte, développement

carbone, 61-62, 117

et eau, 86-87, 180

modèles soutenables, $120-121$

non durabilité, 115, 119-120

ressources naturelles, 116, 119

valeur de la nature, 50

Economie des écosystèmes et de la biodiversité (TEEB),

49-50, 200, 203

économie verte, développement, 115-121

gestion des ressources, 119

incitants de la conservation, 117-118

mesure de la durabilité, 116

modèles économiques alternatifs, 119-121

valorisation de l'environnement, 115-116

écosystèmes. voir aussi services écosystémiques

besoins humains, 37-38

catastrophes naturelles, 101-105

changements climatiques, 56-57

EbA, 59-60

Edouard, lac, 95-96

efficacité énergétique, 84

égalité entre les sexes, 204-205

Eliasch Review, 58

empreinte hydrique, 177

énergie, 83-90

alternatives, $84-88$

biomasse, 85-86

conservation, 84, 90

consommation par les TI, 124

coûts et bénéfices, 89

développement durable, 88-90

eau, 181

efficacité, 84

éolienne, 87

géothermique, 88

hydroélectrique, 86-87

nucléaire, $84-85$

solaire, 88

vagues et marées, 88

entreprises. voir aussi secteur privé

engagement dans la conservation, 145-149

impacts sur la biodiversité, 143

performance environnementale, 148-149

RSE, 140, 145, 148

souci de durabilité, 145

« verdissement ", 149

environnement

accords internationaux, 134-135

capacité de charge, 33 
catastrophes naturelles, 101-105

changements par l'homme, 202

et développement, 20-26, 138, 200

dynamique, 19-20

et femmes, 205

intégration dans tous les secteurs, 200-201

lois et réglementations, 140

opinion publique, 137

réunions futures, 138-139

santé humaine, 110

valorisation économique, 116-117, 203

éolienne, énergie, 87

équité, 204-205

espaces verts, villes, 192-193

espèces, 73-80

et agriculture, 184, 189

avenir de la conservation, 80

changements climatiques, 54-56, 161-162

commerce international, 76, 77

conflits hommes-faune, 78

connaissances, lacunes, 73-75

conservation, basée sur les, 73

découvertes d', 96

eau douce, 175

études grâce aux TI, 125

information et identification, 74

parents sauvages, 184

pêche, 161

santé humaine, 108-110

utilisation durable, 75-78

villes, 191, 196

zones arides, 168

espèces domestiquées, 184

espèces envahissantes, $56,78-80$

aires protégées, 67

biocarburants, 85

et biodiversité, 78

catastrophes naturelles, 102-103

eau douce, 175-176

éradication et contrôle, 80

et espèces menacées, 79

oiseaux, 79

villes, 191

espèces menacées, 79

espèces non indigènes, 67

espèces protégées, viande de brousse, 95

État des Récifs coralliens du monde, 56

États des forêts du monde 2009, 151

éthique de la conservation, 29-34

action collective, $31-33$ outils, 33

responsabilité individuelle, $32-33$

valeurs, 29-31

Évaluation de la dégradation des terres dans les zones arides (LADA), 171

Évaluation des écosystèmes pour le millénaire (EM), 115 agriculture, 183

biodiversité, 18

espèces envahissantes, 78-79

services écosystémiques, 20, 37, 45, 46

zones arides, 168

événements extrêmes. voir catastrophes naturelles

faim, 20-21

FAO

accords internationaux, 135

agriculture, 189

faim, 20-21

forêts, 151, 156

pêche, 77, 160-161

zones arides, 171

faune sauvage, 78, 94-96, 111-113

femmes

agriculture, 188-189

égalité des sexes, 204-205

gestion de l'eau, 180

rôle dans la conservation, 205

fertilisation de l'océan, 129, 160, 162-163

financement et capacité, 68-69, 136

flux d'eau, 86, 104, 178

flux environnemental, 178

Fondation Asahi Glass, 137

Fonds pour l'eau de Quito, 180

Fonds pour l'environnement mondial (FEM), 136

Forest Trends, 156

forêts. voir aussi systèmes forestiers

et agriculture, 153

changements climatiques, 58, 156-157

déclin mondial, 151

gestion durable, 152, 154, 155

gouvernance, $154-155$

restauration, 152-154, 157

utilisation durable, 94

Forum de Barcelone. voir Forum mondial de la nature

Forum d'évaluation de la durabilité hydroélectrique, 86

Forum mondial de la nature. voir aussi Congrès mondial de la nature (CMN) 2008

Centre de ressources, 10

espèces, 73

et secteur des affaires, 143 
thèmes, 15-16

frontières, 180. voir aussi aires protégées transfrontalières fumée, effets sur la santé, 88-89

gaz à effet de serre (GES), 53-54, 124, 191

génétique, 125-126. voir aussi organismes génétiquement modifiés

gens et individus. voir aussi communautés locales

droits, 40

nature et villes, 192, 195-196

responsabilité environnementale, 32-34

rôle dans la conservation, 40-43

services écosystémiques, 37-38, 46

géo-engineering, 129-130, 160

géothermie, 88

gestion

aires protégées, $66-68$

approche inclusive, 207

déchets post-catastrophes, 103

eau et flux, 178, 180, 181

grâce aux TI, 125

océans, 163

pêches, 161-162

programmes de conservation, 41-43

ressources naturelles, 77, 118, 119

zones arides, 171-172

gestion adaptative, 202, 203

gestion durable des forêts (GDF), 152, 154, 155

gestion intégrée des ressources en eau (GIRE), 176, 178, $179,180,181$

Global Diversity Outlook 2 (GBO2), 17-19

Globescan, 115

gomme arabique, 170

Google Earth et Google Ocean, 66, 163

gouvernance

aires protégées, 69

eau, $178-180$

efficacité, 203-204

forêts, 154-155

océans, 162-163

zones arides, 172

gouvernements, 61, 96-97, 200

Great Limpopo, 111-112

Greendex, 115-116

Groupe CSE/UICN de spécialistes de l'utilisation durable, 76

Groupe CSE/UICN de spécialistes des espèces envahissantes (GSEE), 79

Groupe de travail inter-commissions sur l'Éthique en conservation, 29
Groupe d'experts intergouvernemental sur l'évolution du climat (GIEC), 53, 157

guerre. voir conflits armés

hauts-lieux de la biodiversité, 27, 93

hippos, 95

humanité, 31-33, 137

hydroélectricité, 86-87, 181

îles, changements climatiques, 57-58

incitants économiques de la conservation, 117-118

Inde, 94

Index Planète vivante, 33

indicateurs, biodiversité, 17-18

Indice de la Liste rouge pour les oiseaux, 75, 175

Initiative Mangroves pour l'avenir, 58

Initiative taxonomique mondiale (ITM), 75

Institut international pour l'environnement et le développement (IIED), 156

Intensively Managed Planted Forests: towards best practice, 155

Inventaire tous terrains, tous taxons, 74

Investissement direct à l'étranger (IDE), 136-137

investissements, gestion de l'eau, 181

jeunes, et aires protégées, 70

Katrina, ouragan, 102-103

langues, disparition, 202

Le passage à la durabilité : vers un monde bumain et divers, 207

Lignes directrices pour l'application de catégories de gestions aux aires protégées, 66

Lignes directrices pour les sites naturels sacrés, 67

Liste Rouge de l'UICN des espèces menacées 2008, 17, 74-75, 126, 151, 175

lois

eau, gestion, 178-179

forêts, 154-155

lois internationales

conflits armés, 98-99

environnement, 140

Londres, biodiversité, 192

maladies

aires protégées transfrontalières, 111-113

contrôle et effets sur l'environnement, 110

échanges et dispersion, 109, 110, 111

marché du carbone, 117

marchés alimentaires, viande de brousse, 95

marchés économiques 
grumes, 154-155

incitants pour la conservation, 117-118

services écosystémiques et PES, 48-49, 51, 118

marées et vagues, énergie des, 88

Mécanisme pour le développement propre (MDP), 117 microorganismes, 109-110, 130

migration animale, et conflits armés, 96

mondialisation, impact environnemental, 38

monocultures, $187-188$

moyens de subsistence, forêts, 155-156

nanotechnologies, 126-127

National Geographic, 115

Nations Unies, 162, 176

nature

modèle technologique, 126-127

valeur, 50, 192-194

Naxalites, 94

Népal, conflits et conservation, 97

Norme internationale pour la collecte sauvage soutenable de plantes médicinales et aromatiques (ISSC-MAP), 111

nourriture, production et demande, 183

nucléaire, énergie, 84-85

Objectif Biodiversité 2010, 17-20

Objectifs du millénaire pour le développement (OMD) accords internationaux, 138

changements climatiques, 60

eau, 104

environnement, 23-26, 203

faim, 21-22

services écosystémiques, 48

océans et mers. voir aussi systèmes marins

aires marines protégées, 66, 162, 163-164

changements climatiques, 159-160

fertilisation, 129, 160, 162-163

gestion, 163

gouvernance, 162-163

marées et vagues, énergie des, 88

oiseaux, 79, 87, 191-192, 193

ONU-Habitat, 191

Organisation de coopération et de développement économiques (OCDE), 119

OCDE-CAD, 136

Organisation météorologique mondiale (OMM), 171

Organisation mondiale de la santé (OMS), 111

Organisation mondiale du commerce (OMC), 162

Organisation mondiale du tourisme (OMT), 144

organismes génétiquement modifiés (OGM), 128-129

Ouganda, 95 outils

aires protégées, 66

éthique de la conservation, 33

ressources en eau, 176

paiements pour les services écosystémiques (PES), 47

collaboration internationale, 136

eau douce et bassins, 180-181

économie verte, $117-118$

forêts, 154

législation, 140

paix, et création d'aires protégées, 96-98

Parc national de la Garamba, 95

Parc National de Yala, 102

Parc national des Virunga, 95-96

parcs nationaux, 69, 96-97, 192. voir aussi aires protégées parcs pour la paix, $96-98$

Partenariat de collaboration sur les forêts (CPF), 156

Partenariat mondial pour les villes et la biodiversité, 192

Partenariat pour les Critères mondiaux pour un tourisme durable (GSTC), 144

Partenariat relatif aux indicateurs de biodiversité 2010 (BIP), 17-18

partenariats, 146-149, 155-156

Partenariats forestiers en développement (PFD), 156

Parties à la CDB, aires protégées, 69

pastoralisme, 168

pauvreté

et changements climatiques, 60

et forêts, 151

hauts-lieux de la biodiversité, 93

statut en 2008, 20

pauvreté, réduction

et conservation de la biodiversité, 20, 23

et environnement, 201

et services écosystémiques, 20-23

Tanzanie, 201

pays en développement, 21-23, 88-89, 136

pêche

braconnage, 95-96

changements climatiques, 160

énergie éolienne, 87

espèces, 161

gestion, 161-162

subsides, 118

surpêche, 77, 160-162

pétrole, alternatives, 83-84

Philippines, 94

PIB, et financement de l'APD, 136

planification, 102-105, 202 
plantes, et agriculture, 184-185

plantes médicinales, 108, 110-111

poissons, statut en eau douce, 175-176

politique

coopération internationale, 137-139

gestion de l'eau, 179

restauration post-catastrophes, 104

réunions futures sur l'environnement, 139

et services écosystémiques, 48-49

zones arides, 172

Politique d'équité entre les sexes, 204-205

population

et cours d'eau, 180

espaces verts, 193

mondiale, 24-27

santé humaine, 107

villes, 201

principe de précaution, 33, 126, 131

Principe de Rio 10, 207

Principes de Dublin, 178

Programme des Nations Unies pour l'environnement (PNUE), 38, 102, 135

Programme Marin, 66

Programme mondial sur les espèces envahissantes (GISP), 79,85

Programme sur la restauration des paysages forestiers, 39

Protocole de Carthagène, 129

Protocole de Kyoto, 117

Protocole de Montréal, 31-32

Ramsar (Convention de), 61, 134

Rapport mondial sur le développement humain 2008, 20 récifs coralliens, 159

Réduction des émissions issues de la déforestation et de la dégradation des forêts (REDD), 49, 58-59, 155, 156

reforestation, aux Philippines, 94

Registry of Standard Biological Parts, 130

réglementation environnementale, 140

République Démocratique du Congo, 95

Réseau des flux environnementaux, 178

Réseau mondial des aires protégées, 97

Réserve forestière de Mabira, 153

responsabilité sociale des entreprises (RSE), 140, 145, 148

ressources naturelles

conflits civils, 93-94

et consommation, 38

et économie, 116, 119

gestion, 77, 118, 119

pays en développement, 21-23 restauration

catastrophes naturelles, 101-105

forêts, 152-154, 157

rivières et bassins, $86,117-118$

Royaume-Uni, 88, 124

Sakhalin Energy, 148

Santé animale pour l'environnement et le développement (AHEAD), 111-113

santé humaine, 107-111

biodiversité, importance, 107-108, 110-111, 113

connaissances traditionnelles, 108-109

contribution des espèces, 108-110

microorganismes, 109-110

OGM, 129

pertubation d'écosystèmes, 110

plantes médicinales, 108-109

et santé animale, 111-113

services écosystémiques, 110

Sauver la planète, 29, 31-32

savoirs traditionnels, 39-40, 108-109

secteur privé, collaboration, 140, 143-149

avantages, 148-149

durabilité, 200

établissement des relations, 146-148

partenariats pour la conservation, 146-149

Service d'information sur les espèces de l'UICN (SIS), 74

Service pour la survie des espèces, 73

services écosystémiques, 45-51. voir aussi paiements pour

les services écosystémiques (PES)

agriculture, 45

changements climatiques, 57

classification, 46-48

concept, 20

conservation, modèle pour la, 199

culture, 46, 47-48

dynamique, 19-20

eau douce, 176-177

évaluation, 49-51

inclusion dans l'économie, 48-49, 51, 118

et pauvreté, 20-23

politique et planification, 48-49

restauration post-catastrophes, 103

rivières et bassins, 117-118

santé humaine, 110

utilité des TI, 125

valeur et bénéfices, 47-48, 49-50

zones arides, 170-171

Sites naturels du patrimoine mondial, 65

sites sacrés, 67 
Situation mondiale des pêches et de l'aquaculture, 160-161

solaire, énergie, 88

Sommet de la Terre, 32, 33, 48

Sommet mondial du développement durable (SMDD), $17,48,138,179$

Soudan, 96, 170

Species 2000, 75

Sri Lanka, 102

Stern, rapport, 58, 59

stockage de carbone (CSC), 160, 171

Stratégie et le Plan d'action nationaux pour la

biodiversité, 189

Stratégie mondiale de la conservation, 29

subsides et incitants fiscaux, 118

Sudd, migration animale, 96

surpêche, 77, 160-162

Système mondial d'information sur la biodiversité, 74

systèmes agricoles, 183-189. voir aussi agriculture

habitats naturels, 185-186

imitation des écosystèmes naturels, 186-189

production agricole, 184-186

systèmes arides, 167-172

agriculture, 168

biodiversité, 168, 169

changements climatiques, 171

désertification, 168-169, 171

distribution mondiale, 167

eau, gestion, 171-172

gouvernance, 172

problèmes, 169-171

services écosystémiques, 170-171

systèmes d'eau douce, 175-181

changements climatiques, 181

espèces envahissantes, 175-176

gestion et flux, 178, 181

gouvernance, $178-180$

services écosystémiques et PES, 176-177, 180-181

systèmes forestiers, 151-157. voir aussi forêts

changements climatiques, 156-157

coupes illégales, 154-155

lois et gouvernance, 154-155

moyens de subsistance, 155-156

restauration, 152-154

systèmes marins, 159-164. voir aussi océans et mers

aires marines protégées, 66, 162, 163-164

changements climatiques, 159-160

gouvernance, 162-163

surpêche, 77, 160-162

systèmes urbains, 191-197

espaces verts et aires protégées, 192-193 espèces, 191, 196

impacts, 201

nature et biodiversité, 194-197

valeur de la nature, 192-194

Table ronde sur les biocarburants durables, 86

Tanzanie, 201

taxonomie, 74-75

technologie de l'information

bénéfices pour la conservation, 125

et biodiversité, 125, 126

impacts sur l'environnement, 124

technologie et conservation, 123-131

biologie synthétique, 130-131

biomimétisme, 127-128

biotechnologies, 126-131

géo-engineering, 129-130

impacts, 123

nanotechnologies, 126-127

OGM, 128-129

technologies de l'information, 124-126

technologies traditionnelles, 123

Tematea, 134

température, hausse, 55

termite souterrain de Formose, 102-103

Terre-Neuve et Labrador, 207

territoires traditionnels, et biodiversité, 40-41

tigres, 94

tilapia, 95-96

tourisme durable, 144

TRAFFIC, 111

UICN

accords internationaux, 134-135

besoins humains, 38

biodiversité culturelle, 39

changements climatiques, 58

CMAP, 163

communautés locales, 206-207

conflits armés, 96, 98, 99

droits, 40, 205

eau, 176, 178, 179

économie verte, 121

énergie, 85-86, 89

équité, 204-205

espèces, 184

éthique, position, 31

gouvernance, 155-156, 204

hydroélectricité, 86

industries extractives, 143 
OGM, 128-129

PFD, 156

plantes médicinales, 111

secteur privé, 146, 148

tourisme durable, 144

villes, 192

vision et mission, 204

zones arides, 167-168

Union européenne (UE), 77

utilisation durable

espèces, $75-78$

gestion, 77

produits forestiers, 94

vagues et marées, énergie des, 88

valeur

de l'environnement, 116-117, 203

de la nature, 50, 192-194

services écosystémiques, 47-48, 49-50

valeurs

et consommation, 38

éthique et conservation, 29-31

vent, énergie, 87

Venter, J. Craig et l'Institut Venter, 130

Via Campesina, 129

viande de brousse, 95

vie sauvage, commerce, 76, 77

villes. voir systèmes urbains

Vision de l'eau et de la nature, 176

Water footprint, 177

Wildlife Conservation Society, 96, 111

World Energy Outlook 2008, 83

WWF (Fonds mondial pour la nature), 111, 144

Yémen, 189

zones arides. voir systèmes arides

Zones clé pour la biodiversité (KBA), 66 


\section{Crédits photos :}

Page 7 (C) UICN/Sue Mainka • Page 8 ( Daria Motorna/Dreamstime.com • Page 11 ( ) Martin Harvey/Still Pictures • Page 12 (C) Corbis • Page 14 (C) R. Gemperle/Still Pictures • Page 27 (C) Jack Dykinga/ naturepl.com • Page 28 (C) Flikr/daveblume $\bullet$ Page 35 ( Reuters/Donald Chan • Page 36 (C) Corbis • Page 42 (C) Reuters/Enrique Castro-Mendivil • Page 44 (C) Earl \& Nazima Kowall/Corbis • Page 51 (C) Biosphoto/Christophe Courteau/Still Pictures • Page 52 @ Paul Marshall • Page 63 @ Outdoorsman/ Dreamstime.com • Page 64 (C) Massimo Ripani/Grand Tour/Corbis • Page 71 (c) Franck Charton/ Corbis • Page 72 @ Tim Davis/Corbis • Page 75 @ UICN/Sue Mainka • Page 81 C McPHOTO/Still Pictures $•$ Page 82 (C) Mirounga/Dreamstime.com • Page 91 (C) Daniel Deitschel/Getty images $•$ Page 92 (C) Reuters/Carlos Barria • Page 97 (C) Reuters/Pool/Themba Hadebe • Page 98 (C) Jung Yeon-Je/ Getty images • Page 100 @ Reuters/Kamal Kishore • Page 106 @ UICN/Sue Mainka • Page 112 @ R Gemperle/Still Pictures • Page 114 (C) Corbis • Page 120 (C) Nigel Dickinson/Still Pictures • Page 122 (C) Dave Watts/naturepl.com • Page 128 (C) Uwe Wittbrock/Fotolia.com • Page 132 (C) Markus Seidel/ iStockphoto • Page 139 (C) Alex Nikada/iStockphoto • Page 141 (C) A. Ishokon-UNEP/Still Pictures • Page 142 (C) Manfred Vollmer/Das Fotoarchiv/Still Pictures • Page 147 (C) Reuters/Luke Distelhorst • Page 150 (C) Wild Wonders of Europe/Döerr/naturepl.com • Page 157 (C) Andy Rouse/naturepl.com • Page 158 (C) Reinhard Dirscherl/WaterFrame/Still Pictures • Page 165 (C) Reuters/Babu Babu • Page 166 (C) Iconotec • Page 173 (C) Digital vision • Page 174 (C) Biosphoto/Robert Valarcher/Still Pictures • Page 182 (C) Reuters/Sonam Wangdi • Page 189 @ Reuters/China Photos • Page 190 @ El Fotopakismo/ Flikr • Page 197 (C) Elizabeth Brixton/Flikr • Page 198 (C) Aflo/naturepl.com• Page 207 (C) Galdzer/ Dreamstime.com 


\section{UICN}

UNION INTERNATIONALE POUR LA CONSERVATION DE LA NATURE

SIĖGE MONDIAL

Rue Mauverney 28

1196 Gland Suisse

Tél +4122999 0000

$\mathrm{Fax}+41229990020$

www.iucn.org

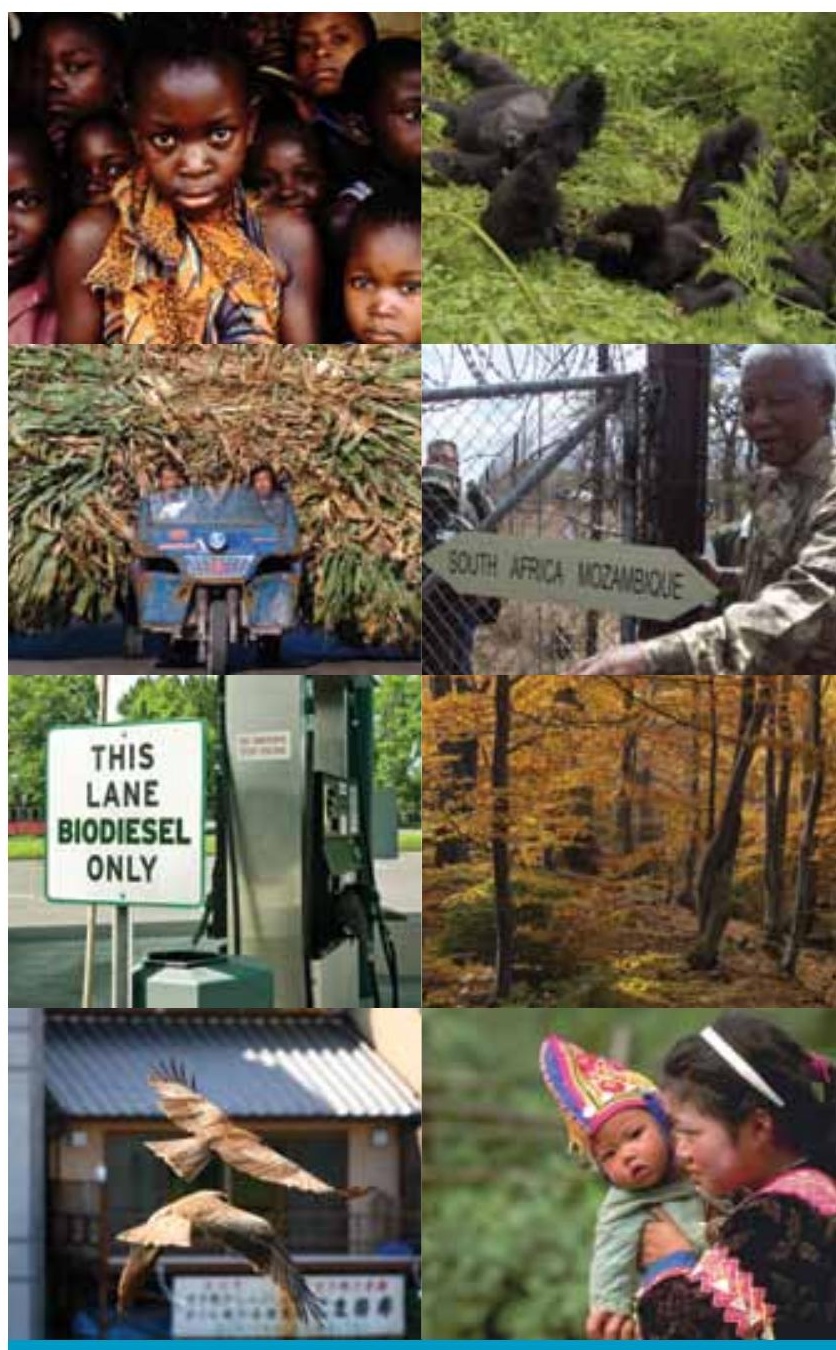

